\title{
Neuartiger Ansatz zur Untersuchung des tribologischen Systems Ventilspindel/Sitzring für Großgasmotoren
}

Von der Fakultät für Ingenieurwissenschaften, Abteilung Maschinenbau und Verfahrenstechnik der

Universität Duisburg-Essen

zur Erlangung des akademischen Grades

eines

Doktors der Ingenieurwissenschaften

Dr.-Ing.

genehmigte Dissertation

von

Oliver Lehmann

aus

Frankfurt (Oder)

1. Gutachter: Prof. Dr.-Ing. habil. Alfons Fischer

2. Gutacher: Prof. Dr.-Ing. habil. Matthias Scherge

Tag der mündlichen Prüfung: 24.10.2018 



\section{Danksagung}

Die vorliegende Dissertation entstand anfangs während meiner Tätigkeit als Projektingenieur und später zum Großteil neben meiner Arbeit als Leiter der Abteilung Forschung und Entwicklung bei der Märkisches Werk GmbH.

Für die hierfür geleistete wertvolle Mitarbeit möchte ich allen Beteiligten danken. Besonderen Dank gebührt der Märkisches Werk GmbH für die großzügige finanzielle Förderung. Herrn Dr.Ing. Holger Fellmann, ehemaliger Leiter der Abteilung Forschung und Entwicklung bei der Märkisches Werk $\mathrm{GmbH}$, danke ich herzlich für die Förderung und Unterstützung des Projekts.

Bei Herrn Prof. Dr.-Ing. habil. Alfons Fischer, Lehrstuhl für Werkstofftechnik des Instituts für Technologien der Metalle an der Universität Duisburg-Essen, möchte ich mich für die thematische Herangehensweise und seine stete Gesprächsbereitschaft, die im Ergebnis erst die Behandlung des Themas in dieser Art und Weise ermöglichte, sowie für die Übernahme des Hauptreferats bedanken.

Herrn Prof. Dr.-Ing. habil. Matthias Scherge, Leiter Geschäftsfeld Tribologie am Fraunhofer IWM und Leiter MikroTribologie Centrum $\mu \mathrm{TC}$, danke ich für die wohlwollende Unterstützung bei der Entstehung und Umsetzung dieser Arbeit sowie für die Übernahme des Korreferats.

Allen Institutsmitgliedern am Fraunhofer IWM, die mir bei der Entwicklung, Aufbau und Inbetriebnahme des Ventilverschleißprüfstands sowie bei der Durchführung der Experimente unterstützt haben, sei an dieser Stelle gedankt. Mein besonderer Dank gilt Herrn Dr.-Ing. Alexander Renz für seine stete Diskussionsbereitschaft in vielen Gesprächen, Herrn Jan Klipfel, Herrn Jan Speck und Herrn Dr.-Ing. Dominik Kürten.

Großen Dank möchte ich an meine Eltern, Roland und Gerlinde, richten, die mir bereits zu früher Zeit die Möglichkeit einräumten und darin bestärkten, den für mich richtigen Weg zu gehen. Nicht vergessen möchte ich Dr. Tilmann Johannes Heinisch $(\dagger)$, der mich ermutigte und zum Studium Maschinenbau lenkte.

Dank schulde ich meiner Ehefrau Simone, meiner Tochter Helena und meinem Sohn ArthurOliver, die sich vermutlich oft gefragt haben, was ihr Ehemann bzw. Papa eigentlich die ganze Zeit am späten Abend bis in die Nacht und an den Wochenenden so macht. Vielen Dank für Eure Unterstützung und den Freiraum während meiner Promotion, ohne den diese Arbeit nicht so möglich gewesen wäre.

Halver im Oktober 2018 
Ich schreibe dies, wie es mir wahr zu sein scheint.

Hekataios von Milet

(um 550 bis 476 v. Chr.) 


\section{Kurzfassung}

Die technische Entwicklung von Großgasmotoren in den Bereichen Marine, Lokomotive und insbesondere Kraftwerk hat zu mechanischen Wirkungsgraden von über $50 \%$ geführt, wodurch das tribologische System Ventilspindel/Sitzring an den Grenzen seiner Funktionsfähigkeit eingesetzt wird. Problemverschärfend wirkt eine zunehmend strengere Abgasgesetzgebung. Hocheffiziente Großgasmotoren werden im Systemverbund mit neuen Technologiekonzepten als potenzieller Ansatz für einen emissions- und kostenreduzierten Betrieb gesehen. Die steigenden Zünddrücke, höheren Temperaturen und reduzierten Verbrennungsrückstände bewirken aufgrund neuartiger Betriebsbedingungen einen hohen Ventilverschleiß mit vorzeitigem Funktionsausfall.

Das tribologische Verhalten der Paarung Ventilspindel/Sitzring ist nach Quellenlage aus den Bereichen Automotive und Heavy-Duty bekannt. Als wichtigste Einflussfaktoren auf den Ventilverschleiß wurden die beiden Phasen Ventilschließen und Verbrennungsdruck bestimmt. Hingegen sind für den Bereich Großgasmotor, außer vom Autor selbst, bislang keine wissenschaftlichen Arbeiten dokumentiert. Darüber hinaus fehlt eine Zuordnung der eigenschaftsbestimmenden Verschleißmechanismen sowie der Verschleißanteile am Gesamtventilverschleiß für die beiden Phasen Ventilschließen und Verbrennungsdruck.

Die vorliegende Dissertation betrachtet die Entwicklung, Konstruktion und den Aufbau eines neuartigen Ventilverschleißprüfstands zur getrennten experimentellen Simulation der Phasen Ventilschließen und Verbrennungsdruck für Großgasmotoren. Zu diesem Zweck wurden im ersten Schritt Ventilspindeln mit unterschiedlicher Laufzeit aus demselben Typ Großgasmotor mikrostrukturell und chemisch untersucht. Die Untersuchung der tribologisch beanspruchten Randschichten diente zur Analyse der Verschleißmechanismen und der Validierung des Prüfstands in Bezug auf die eigenschaftsbestimmenden Verschleißmechanismen. So konnten aus den ersten Versuchsreihen zum Einfluss von Ventilschließen und Verbrennungsdruck bei $380^{\circ} \mathrm{C}$ an Luft gleich neue Erkenntnisse gewonnen werden. Die Versuche bestätigen den signifikanten Einfluss von Schließgeschwindigkeit und Verbrennungsdruck auf den Ventilverschleiß. Als neue Erkenntnisse konnten eine größere Zunahme des Sitzringverschleißes im Vergleich zur Ventilspindel unter Ventilschießen und eine Abnahme des Ventilspindelverschleißes um ca. 30 \% trotz einer Steigerung des Verbrennungsdrucks von 140 auf 180 bar gewonnen werden. Dieser Verschleißrückgang mit steigender Last bedeutet, dass die mechanisch-dominierten Verschleißmechanismen unter Ventilschließen durch chemisch-dominierte unter Verbrennungsdruck überlagert werden. Ferner konnten die beiden Verschleißmechanismen Adhäsion mit Werkstoffübergang und Oberflächenzerrüttung mit dem Untermechanismus Delamination beiden Belastungsphasen zugeordnet werden. Die Verschleißraten beider Phasen waren ähnlich groß. Als vierte, wichtige und neue Erkenntnis folgt, dass das Ventilschließen zur Bewertung des Systemverhaltens wichtiger ist als der Verbrennungsdruck. Diese neuen Erkenntnisse, die beispielhaft mit den Werkstoffen Stellite ${ }^{\mathrm{TM}}$ 12 bei der Ventilspindel und Pleuco $12 \mathrm{MW}$ beim Sitzring untersucht wurden, können jetzt als belastbare Ansatzpunkte für weitere Parameterstudien und Werkstoffentwicklungen genutzt werden. 


\section{Abstract}

The technical development of large bore gas engines for marine, locomotive and power plant in particular leads to mechanical efficiencies of more than $50 \%$, whereas the tribological system valve spindle/seat ring is applied at its functional limits. Stringent emission regulations has an additional effect. Highly efficient large bore gas engines combined with new technology concepts are a promising approach in order to reach lower emissions and operating costs. Because of novel operating conditions caused by increasing peak firing pressures, higher temperatures and reduced combustion residues valve wear effects premature failure.

Based on findings from literature the tribological behavior of the pair valve spindle/seat ring is known from automotive and heavy-duty applications. Valve closure and peak firing pressures have been identified as main factors influencing valve wear. However, so far scientific investigations regarding valve wear in large bore gas engines have not been published, apart from the author's papers. Furthermore, an allocation of determining wear mechanisms and a partition of wear on the entire valve wear concerning valve closure and peak firing pressure are missing.

This dissertation deals with the development, design and construction of a novel valve wear test rig for separate simulating of valve closure and peak firing pressure. For this purpose, the microstructure and chemistry of valve spindles with different operating hours from engine tests of the same type of engine were analyzed. Objectives of the investigations were to identify the wear mechanisms and to validate the test rig regarding the determining wear mechanisms. From the first test series on the impact of valve closure and peak firing pressure at $380^{\circ} \mathrm{C}$ on air new findings could be gained. Tests verify the main influence of valve closure and peak firing pressure on valve wear. As new findings an increase of seat ring wear compared to valve spindle for valve closure and a decrease of valve spindle wear from 140 to 180 bar of about $30 \%$ could be found. This decrease by increasing load means that mechanically-dominated wear mechanisms for valve closure are superimposed by chemicallydominated ones. Furthermore, both wear mechanisms adhesion by material transfer and surface fatigue by delamination can be allocated to both phases valve closure and peak firing pressure, while the wear rates of both phases are equal. The fourth new and important outcome is, that valve closure is more important than peak firing pressure in order to evaluate the tribological behavior of valve spindle/seat ring.

This new findings, exemplary examined for materials Stellite ${ }^{\mathrm{TM}} 12$ for valve spindle and Pleuco $12 \mathrm{MW}$ for seat ring, will now provide a better understanding and sustainable propositions for further parameter studies and material development. 


\section{Inhaltsverzeichnis}

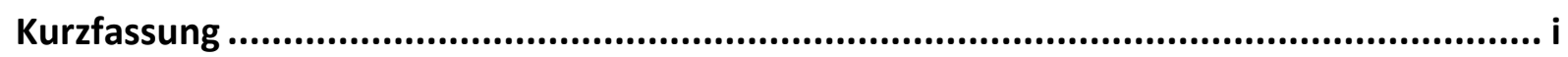

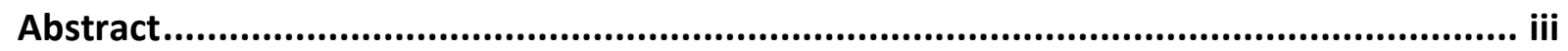

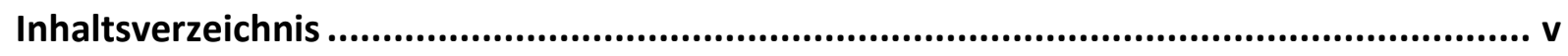

Abbildungsverzeichnis..........................................................................................ii

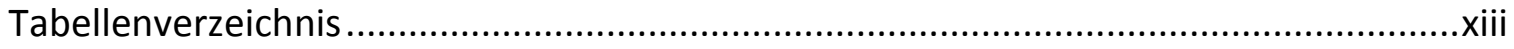

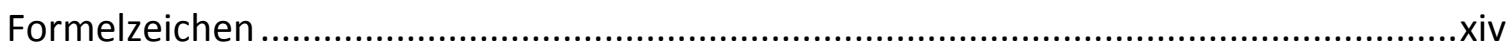

Abkürzungsverzeichnis ....................................................................................

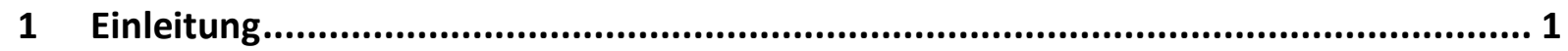

1.1 Motivation des neuartigen Ventilverschleißprüfstands....................................... 1

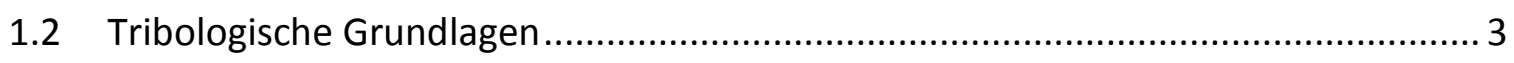

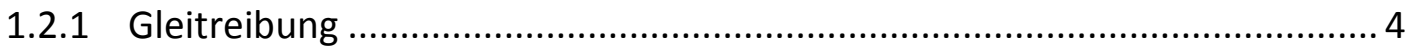

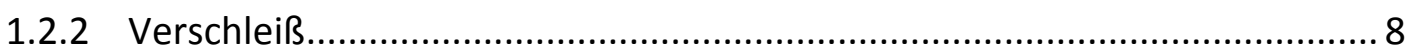

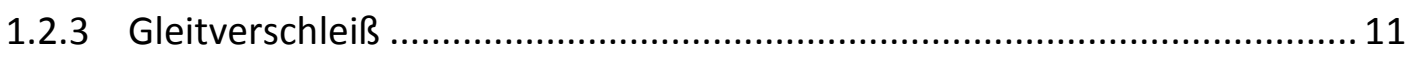

1.2.4 Gleitverschleiß bei Hochtemperatur ...................................................... 14

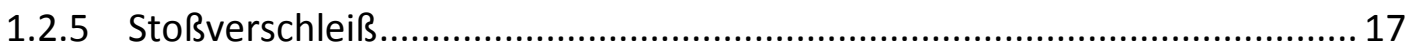

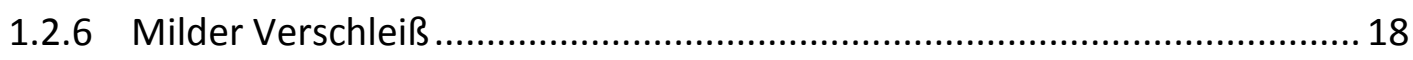

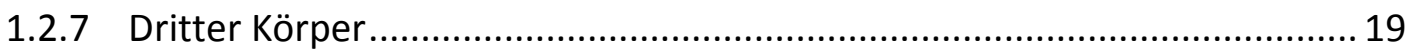

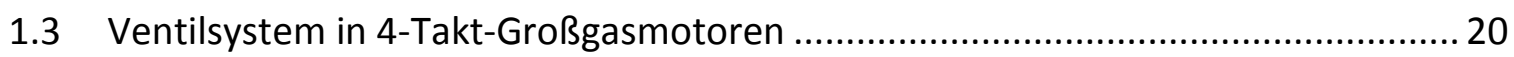

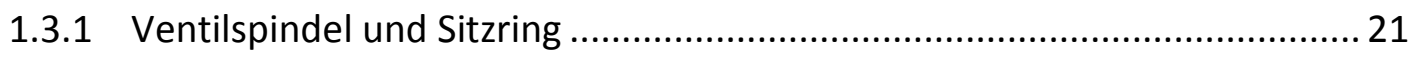

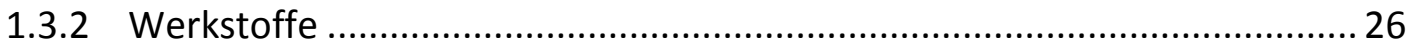

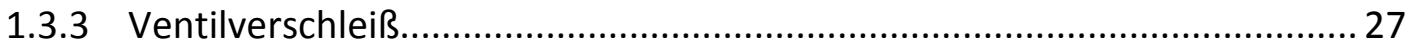

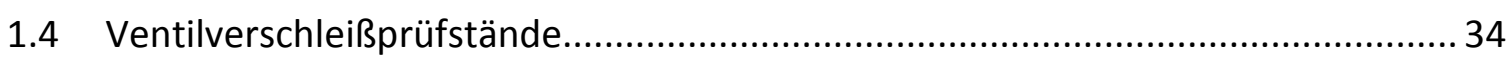

1.5 Zukünftige Trends in der Großgasmotorenentwicklung ...................................... 39

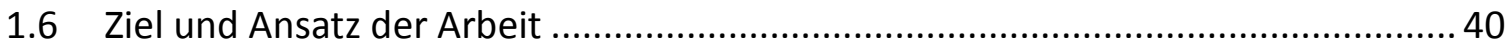

2 Untersuchung der Verschleißmechanismen zur Prüfstandsentwicklung .................. 43

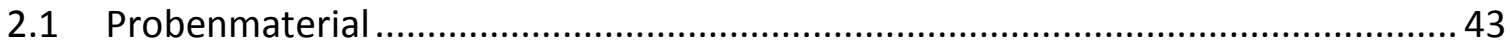

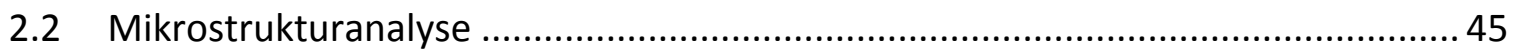

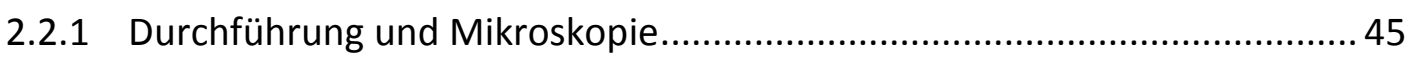

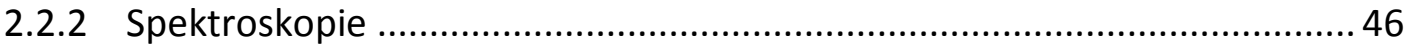




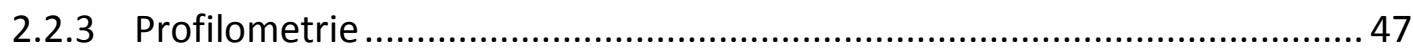

2.3 Stellite $^{\mathrm{rm}} 12-$ Ventilspindeln mit Verschleißraten von bis zu $11,81 \mathrm{~mm}^{3} / \mathrm{h}$............ 49

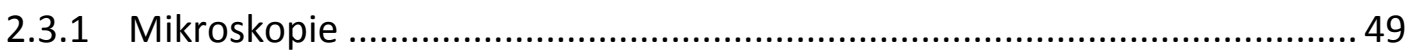

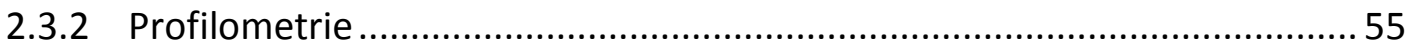

2.3.3 Röntgenphotoelektronenspektroskopie.............................................. 57

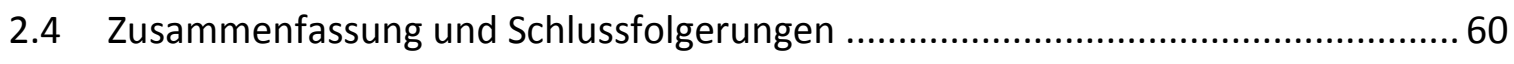

3 Entwicklung, Konstruktion und Aufbau eines neuartigen Ventilverschleißprüfstands 65

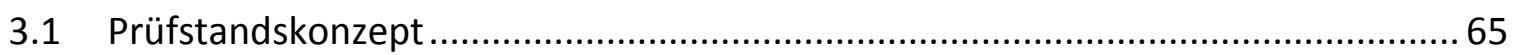

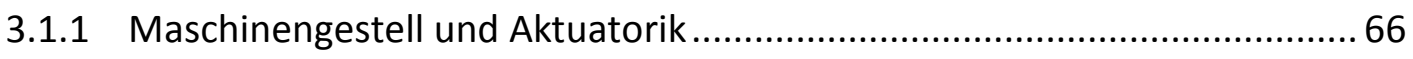

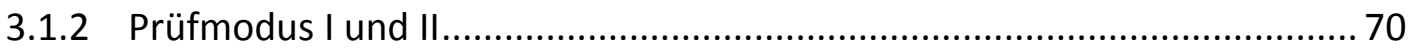

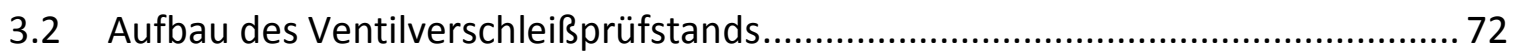

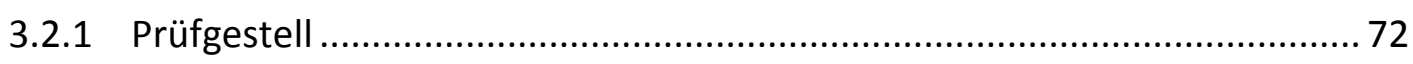

3.2.2 Tastversuche zur Induktionsheizung ................................................... 74

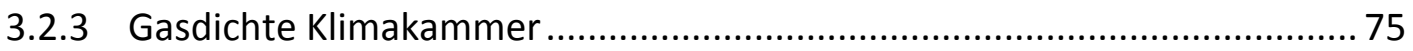

3.2.4 Prüfmodus I - Simulation des Ventilschließens........................................... 76

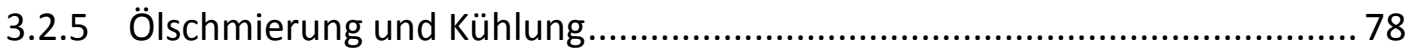

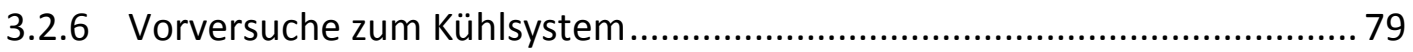

3.2.7 Prüfmodus II - Simulation des Verbrennungsdrucks ............................... 81

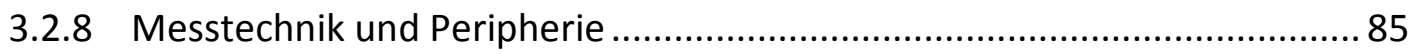

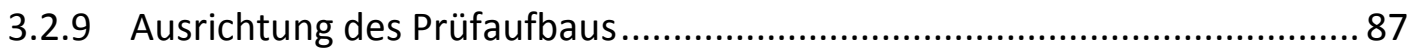

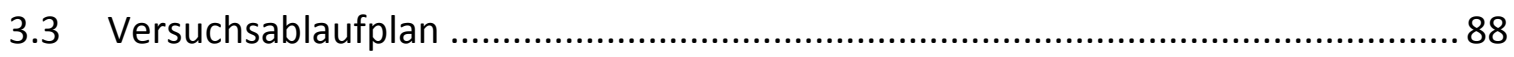

3.4 Validierung des Ventilverschleißprüfstands ................................................... 89

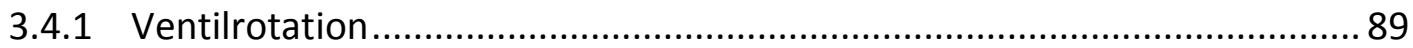

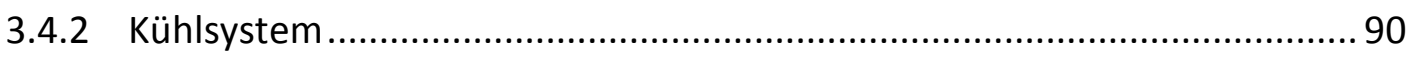

3.4.3 Verschleißerscheinungsformen ........................................................... 92

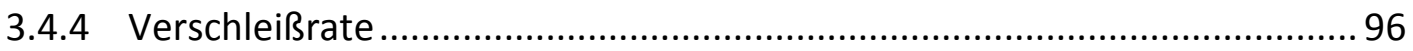

4 Erste Ergebnisse aus den Prüfstandsversuchen .................................................... 97

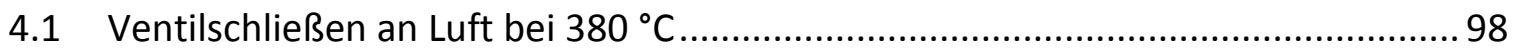

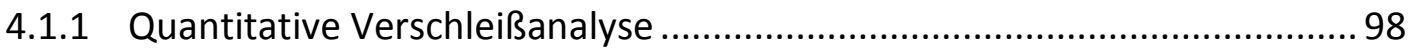

4.1.2 Mikrostrukturanalyse ..................................................................... 101

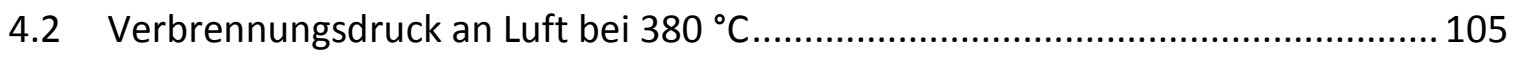

4.2.1 Quantitative Verschleißanalyse ....................................................... 105

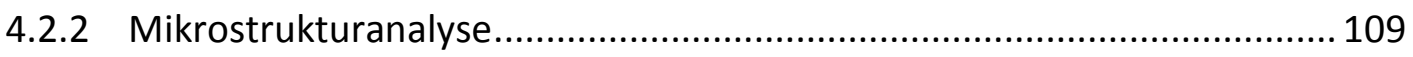




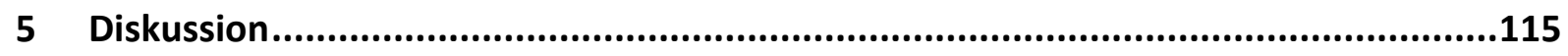

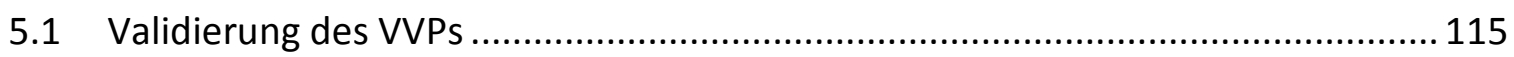

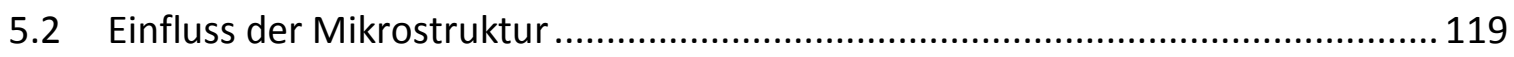

5.3 Neue Erkenntnisse zum Einfluss des Ventilschließens ...................................... 120

5.4 Neue Erkenntnisse zum Einfluss des Verbrennungsdrucks ................................. 122

5.5 Schlussfolgerungen für zukünftige Großgasmotoren....................................... 125

6 Zusammenfassung und Ausblick ......................................................................127

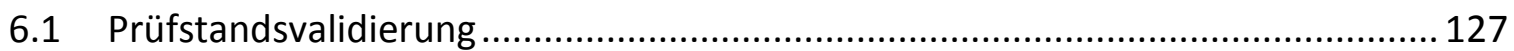

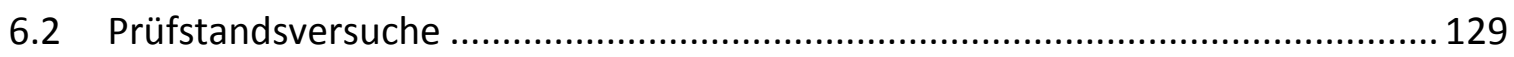

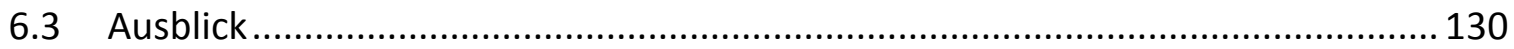

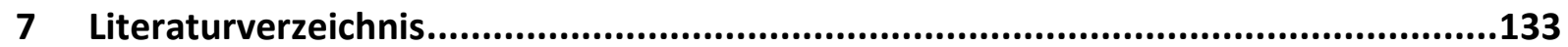

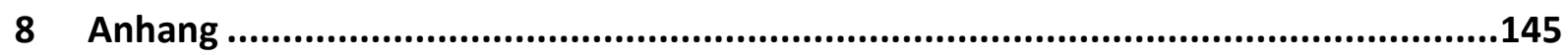




\section{Abbildungsverzeichnis}

Abbildung 1.1: Entwicklung der Verbrennungsspitzendrücke von Dieselmotoren von 1960 bis heute in Anlehnung an [3] .....

Abbildung 1.2: Verschlissene VSn aus hochbelasteten Großgasmotoren.

Abbildung 1.3: Schematische Darstellung eines tribologischen Systems nach [15] ................. 3

Abbildung 1.4: Nominelle und reale Kontaktfläche nach [22] ............................................ 6

Abbildung 1.5: Schematische Darstellung der tribologischen Beanspruchung nach [15]....... 10

Abbildung 1.6: Schematischer Verschleißverlauf als Funktion von Belastungszeit oder

Gleitweg nach [22]

Abbildung 1.7: Schematischer Aufbau technischer Werkstoffgrenzschichten und

Mikrostrukturänderungen unter Gleitverschleiß nach [32] ....

Abbildung 1.8: Schematische Darstellung des Gleitverschleißes mit möglicher

Wiederkompaktierung ausgebrochener Teilchen aus der Glazeschicht nach

[88]

Abbildung 1.9: Qualitativer Härteverlauf über die Tiefe nach [106]

Abbildung 1.10: (a) Zylinderkopf mit montiertem Ventilsystem, (b) Halbschnitt von (a) mit freundlicher Genehmigung der Märkisches Werk GmbH

Abbildung 1.11: Typische Designs für VS und SR; (a) EVS, (b) AVS, (c) ungekühlter ESR und (d) gekühlter ASR mit freundlicher Genehmigung der Märkisches Werk GmbH 22

Abbildung 1.12: (a) Ventilsystem bestehend aus VS und SR, (b) Detail aus (a) mit Differenzwinkel, (c) Detail aus (a) mit Ventilüberstand

Abbildung 1.13: Normierte Darstellung der Ventilerhebungskurven für EVS (rot) und AVS (blau) und Zylinderdruck (grün) über $720^{\circ} \mathrm{KW}$ 24

Abbildung 1.14: Ventilsitz, (a) neu, (b) mit Verschleiß (Ventilrückgang) ..... 28

Abbildung 1.15: Aufschlagskraft als Funktion der Ventilschließgeschwindigkeit. 29

Abbildung 1.16: (a) Valve Bouncing nach [140], (b) außermittiger Sitz aufgrund Nichtkoaxialität von VS- und SR-Achse nach [43].

Abbildung 1.17: Quellen möglicher Edukte zur Bildung einer verschleißminimierenden Triboschicht auf den Sitzkontaktflächen von VS und SR nach [60] 34

Abbildung 2.1: Typisches Gefüge einer PTA-Auftragschweißung von Stellite ${ }^{\text {TM }} 12$;

Vergrößerung 1500 fach

Abbildung 2.2: Probenentnahme, (a) schaftgetrennte VS mit erodierter Probe, (b) Ventilsegment mit drei markierten Untersuchungsbereichen am SID, SM und SAD 45

Abbildung 2.3: Bestimmung der verschlissenen Fläche $A I, x h$. 48

Abbildung 2.4: Probenübersicht, A1 bis A3 mit laufzeitabhängigen ölkohlehaltigen Ablagerungen, (a) A1 ohne erkennbare Ablagerungen, (b) A2 mit 
„Rußspritzern“ im Bereich der Hohlkehle, (c) A3 mit intensiven Ölkohleablagerungen in der Hohlkehle, (d) A4 mit korrodierter Oberfläche und ohne erkennbare Ölkohle

Abbildung 2.5: Übersicht der Ventilsitzflächen, (a) Probe A1 mit linienförmiger Verschleißspur (Linientrag) im Bereich des SADs, (b) Probe A2 mit aufgerauter Kontaktfläche und Verschleißmarkenbreite von ca. $6 \mathrm{~mm}$, (c) Probe A3 mit geglätteter Verschleißspur und Verschleißmarkenbreite über die gesamte nominelle Ventilsitzbreite, (d) Probe A4 mit aufgrauter Kontaktfläche und größerer Verschleißmarkenbreite als nomineller Ventilsitzbreite.

Abbildung 2.6: (a) REM-Detailaufnahme aus Abbildung 2.5 (a) mit einer Glättung der Schleifspitzen infolge des Reibkontakts, (b) Fältelung der Schleifspitzen in radialer Richtung zum SAD mit erkennbaren Schleifriefen; Vergrößerung 400fach

Abbildung 2.7: REM-Draufsichten der Verschleißspur von Probe A2, (a) SID, (b) SM.

Abbildung 2.8: REM-Aufnahmen der Draufsicht aus dem Bereich der SM, (a) Probe A3 mit geglätteter Verschleißfläche und eingedrückten Verbrennungsrückständen sowie Löchern, (b) Probe A4 mit deutlich topografierter Oberfläche und Kuppen, Schuppen sowie Löchern.

Abbildung 2.9: REM-Aufnahmen vom Querschliff, (a) A1 mit Scherbändern, (b) A2 mit Dehnungsgradient bis $60 \mu \mathrm{m}$ Tiefe und mechanisch gemischter Randschicht, (c) A3 mit Dehnungsgradienten, (d) A4 mit parallelen Rissen zur Oberfläche und schuppenförmigem Verschleißpartikel (Delamination)

Abbildung 2.10: REM-Detailaufnahmen, (a) aus Abbildung 2.9 (a) mit Versetzungen und Zwillingsbildung in den metallischen Zellen, (b) aus Abbildung 2.9 (b) mit korngefeinter und metallisch gemischter Randschicht, (c) aus Abbildung 2.9 (c) mit zerbrochenen Karbiden im Dehnungsgradienten, (d) Probe A4 mit Dehnungsgradienten

Abbildung 2.11: Profilometrie an den Ventilsitzflächen der Proben A1 bis A4, SID bei $1,5 \mathrm{~mm}$ und SAD bei $13,7 \mathrm{~mm}$ 55

Abbildung 2.12: Verschleißkenngrößen der Proben A1 bis A4, (a) Verschleißflächen in $\mathrm{mm}^{2}$, (b) Verschleißraten in $\mathrm{mm}^{3} / \mathrm{h}$.

Abbildung 2.13: Atomkonzentrationen in \% als Funktion der Sputtertiefe in $\mathrm{nm}$; XPSMessungen an der Probe A1, (a) Messposition 1 neben der Verschleißspur, (b) Messposition 2 in der Verschleißspur am SAD; Skalierung $60 \%$ 57

Abbildung 2.14: Atomkonzentrationen in \% als Funktion der Sputtertiefe in $\mu \mathrm{m}$; XPSMessungen im Bereich des SADs, (a) Probe A2 und (b) Probe A3; Skalierung $60 \%$. 58

Abbildung 2.15: Atomkonzentrationen in \% als Funktion der Sputtertiefe in $\mu \mathrm{m}$; XPSMessungen, (a) Probe A3 im Bereich des SIDs, (b) Probe A4 im Bereich des SADs ; Skalierung $60 \%$ 
Abbildung 3.1: (a) elektrodynamische Universallösung, (b) servohydraulische Sonderlösung, CAD-Modelle möglicher Prüfaufbauten für (a) und (b) sind (c) bzw. (d) ........66 66

Abbildung 3.2: Parameterstudien zur Vernetzung 69

Abbildung 3.3: Vergleichende Finite Element Analysen zur Tellerdurchbiegung infolge Stempelkraft (a) und Gasdruck (b) .70

Abbildung 3.4: Mind Map als Teil der Konzeptphase von Prüfmodus II. 71

Abbildung 3.5: Schematische Darstellung von Prüfmodus I (a) und Prüfmodus II (b) .... .72

Abbildung 3.6: (a) Prüfrahmen vor Aufbau, (b) Modifikation des linksseitigen Jochs .73

Abbildung 3.7: Prüfrahmen vor (a) und nach (b) der Instandsetzung .73

Abbildung 3.8: (a) Versuchsaufbau der Tastversuche zur induktiven Heizung, (b) TemperaturZeitverlauf am Ventilteller an den drei Positionen Tellermitte (grün), Tellerdurchmesser (schwarz) und SID (rot) bei maximaler Generatorleistung 74

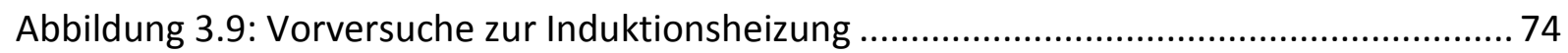

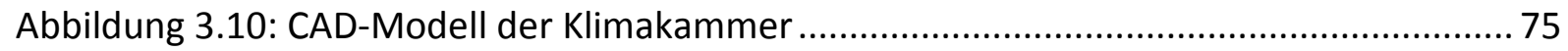

Abbildung 3.11: Klimakammer als CAD-Modell (a) und Fertigteil in Draufsicht (b) ................ 76

Abbildung 3.12: CAD-Modell von Prüfmodus I im Halbschnitt............................................. 76

Abbildung 3.13: CAD-Modelle des Hydraulikzylinders mit Wegmesssystem (a) und der Klimakammer mit Slip-Ring-Transmitter (b) ............................................. 77

Abbildung 3.14: CAD-Modell von Ölschmierung und Kühlung ............................................. 78

Abbildung 3.15: Detailaufnahme der Kühlkreisläufe aus Abbildung 3.14 ............................. 79

Abbildung 3.16: Versuchsaufbau zur validierten Temperaturmessung (a) und überarbeitetes Kühlsystem (b) ... 80

Abbildung 3.17: Validierte Temperaturverläufe an VS (a) und SR (b) ..... .80

Abbildung 3.18: Validierte Temperaturmessungen; (a) Auszug aus einer 5-stündigen Prüfmessung bei verschiedenen Temperaturen bis $600{ }^{\circ} \mathrm{C}$ im Prüfmodus I unter 0,6 m/s, (b) Auszug aus einem Vorversuch im Prüfmodus II unter $130 \mathrm{kN}$ bei $430{ }^{\circ} \mathrm{C}$

Abbildung 3.19: CAD-Modell von Prüfmodus II im Halbschnitt....

Abbildung 3.20: Detailaufnahme aus Abbildung 3.19; Einbausituation von VS/Druckstempel im Halbschnitt als CAD-Modell .

Abbildung 3.21: Hydraulisch-mechanischer Drehmechanismus als CAD-Modell. 83

Abbildung 3.22: Reduzierte Darstellung der Baugruppe Ventildrehmechanismus als CADModell 84

Abbildung 3.23: Messung der Aufschlagskraft als Funktion der Schließgeschwindigkeit im Prüfmodus I am Anfang (a) und nach ca. 4.000 Betriebsstunden (b) ... 86

Abbildung 3.24: Ausschnitt einer Wegmessung, Position des weggesteuerten Hydraulikzylinders im Prüfmodus I über die Zeit von drei Messzyklen. .86 
Abbildung 3.25: Weg- und Kraftmessung des Druckzylinders im Prüfmodus II über einen Zyklus

Abbildung 3.26: Ausrichten von Kraftaufnehmer, Kühlflansch und Sitzringhalter mittels Zentrierwelle und Zentrierring .....

Abbildung 3.27: 3-stufiger Ablaufplan eines typischen $100 \mathrm{~h}$-Versuchs 89

Abbildung 3.28: Validierte Drehmomentmessung zur Ventilrotation über vier Messzyklen im

Prüfmodus I.... 90

Abbildung 3.29: 3-stufig validierte Temperaturmessungen zum Kühlsystem in den beiden Prüfmodi I und II

Abbildung 3.30: Vergleich der Verschleißerscheinungen unter Ventilschließen,

Makroaufnahmen von den Ventiltellern (a) und (b), REM-Aufnahmen von den Draufsichten (c) und (d) sowie den zugehörigen Querschliffen (e) und (f)

Abbildung 3.31 Vergleich der Verschleißerscheinungen unter Verbrennungsdruck, Makroaufnahmen von den Ventiltellern (a) und (b), REM-Aufnahmen von den Draufsichten (c) und (d) sowie den zugehörigen Querschliffen (e) und (f)

Abbildung 3.32: Verschleißprofile unter $0,6 \mathrm{~m} / \mathrm{s}$ bei Raumtemperatur (a) und $430{ }^{\circ} \mathrm{C}(\mathrm{b})$ sowie unter $1,2 \mathrm{~m} / \mathrm{s}$ bei $450{ }^{\circ} \mathrm{C}$ (c) und zugehörige Verschleißraten (d)....... 96

Abbildung 4.1: Verschleißtiefenprofile von VS (links) und SR (rechts) bei 3-stufiger Variation der Schließgeschwindigkeit unter $0,2,0,6$ und $1,0 \mathrm{~m} / \mathrm{s}$ bei $380^{\circ} \mathrm{C}$ an Luft..... 98

Abbildung 4.2: Komponentenverschleißraten von VS und SR für die Versuchsreihe Ventilschließen unter $0,2,0,6$ und $1,0 \mathrm{~m} / \mathrm{s}$ bei $380^{\circ} \mathrm{C}$ an Luft. 100

Abbildung 4.3: Systemverschleißraten zur Versuchsreihe Ventilschließen unter 0,2, 0,6 und $1,0 \mathrm{~m} / \mathrm{s}$ bei $380^{\circ} \mathrm{C}$ an Luft. 100

Abbildung 4.4: REM-Aufnahmen von VS und SR in Draufsicht und Querschliff unter $0,2 \mathrm{~m} / \mathrm{s}$ bei $380^{\circ} \mathrm{C}$ an Luft; 500x (a) - (c) und (g) - (i), 1500x (d) - (f) und (j) - (I) ..... 101

Abbildung 4.5: REM-Aufnahmen von VS und SR in Draufsicht und Querschliff unter $0,2 \mathrm{~m} / \mathrm{s}$ bei $380^{\circ} \mathrm{C}$ an Luft; 500x (a) - (c) und (g) - (i), 1500x (d) - (f) und (j) - (I) ..... 103

Abbildung 4.6: REM-Aufnahmen von VS und SR in Draufsicht und Querschliff unter $1,0 \mathrm{~m} / \mathrm{s}$ bei $380^{\circ} \mathrm{C}$ an Luft; 500x (a) - (c) und (g) - (i), 1500x (d) - (f) und (j) - (I) ..... 104

Abbildung 4.7: Verschleißprofile von VS (links) und SR (rechts) bei 3-stufiger Variation des Verbrennungsdrucks unter 140,180 und 220 bar bei $380^{\circ} \mathrm{C}$ an Luft . 105

Abbildung 4.8: Komponentenverschleißraten von VS und SR der Versuchsreihe Verbrennungsdruck unter 140,180 und 220 bar bei $380^{\circ} \mathrm{C}$ an Luft 107

Abbildung 4.9: Systemverschleißraten zur Versuchsreihe Verbrennungsdruck unter 140, 180 und 220 bar bei $380^{\circ} \mathrm{C}$ an Luft

Abbildung 4.10: Vergleich der Systemverschleißraten beider Ventilphasen Ventilschließen und Verbrennungsdruck 
Abbildung 4.11: REM-Aufnahmen von VS und SR in Draufsicht und Querschliff unter 140 bar bei $380{ }^{\circ} \mathrm{C}$ an Luft; 500x (a) - (c) und (g) - (i), 1000x (d) - (f) und (j) - (I) ... 109

Abbildung 4.12: Detailaufnahme aus Abbildung 4.11 (d) der Triboschicht (links), zugehörige EDS-Analyse in at.-\% des schwarz markierten Rechtecks links (rechts)...... 110

Abbildung 4.13: REM-Aufnahmen von VS und SR in Draufsicht und im Querschliff unter 180 bar bei $380^{\circ} \mathrm{C}$ an Luft; 500x (a) - (c) und (g) - (i), 1000x (d) - (f) und (j) (I)

Abbildung 4.14: REM-Aufnahmen von VS und SR in Draufsicht und im Querschliff unter 220 bar bei $380^{\circ} \mathrm{C}$ an Luft; $500 x(\mathrm{a})-(\mathrm{c})$ und (g) - (i), 1000x (d) - (f) und (j) (I)

Abbildung 4.15: Detailaufnahme aus Abbildung 4.14 (d) mit EDS-Spektren in at.-\% der Triboschicht (1) und mechanisch gemischter Randschicht (2); Vergrößerung 5000x.

Abbildung 5.1: Rot = Metalle, blau = Sauerstoff, Ausgangszustand (oben links), Bildung von Metalloxiden über die Zeit (oben rechts), metalloxidische Triboschicht (unten)

Abbildung 8.1: Bezeichnungen an einer Ventilspindel

Abbildung 8.2: Zusammenbauzeichnung im Halbschnitt von Prüfmodus I 146

Abbildung 8.3: Zusammenbauzeichnung im Halbschnitt von Prüfmodus II 


\section{Tabellenverzeichnis}

Tabelle 1.1: Übersicht dokumentierter Ventilverschleißprüfstände in der wissenschaftlichen Literatur auf Komponentenebene bis 2015 ................................................... 37

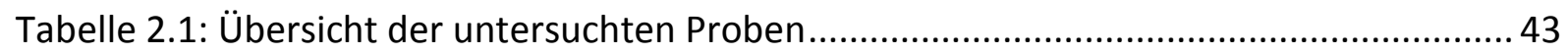

Tabelle 2.2: Nominell chemische Zusammensetzung von Stellite ${ }^{\mathrm{TM}} 12$ in Gew.-\%................... 43

Tabelle 2.3: Nominell chemische Zusammensetzungen der Ventilwerkstoffe in Gew.-\% ...... 44

Tabelle 2.4: Nominell chemische Zusammensetzung von Pleuco $12 \mathrm{MV}$ in Gew.-\% .............. 44

Tabelle 2.5: Vergleich der Sauerstoffkonzentrationen von A3 mit A4 am SAD ....................... 59

Tabelle 3.1: Auswahlkriterien für Universal- und Sonderlösung .........................................67

Tabelle 3.2: Bewertung der Auswahlkriterien für Aktuatoren ................................................69

Tabelle 3.3: Übersicht des Parameterkennfelds für den VVP bezogen auf einen Tellerdurchmesser von ca. $90 \mathrm{~mm}$ bei einer Masse von ca. $1300 \mathrm{~g}$.................... 71

Tabelle 3.4: Minimal- und Maximalwerte an den Messpositionen von VS und SR ................ 91

Tabelle 4.1: Übersicht der ersten zwei Versuchsreihen zum Ventilschließen und

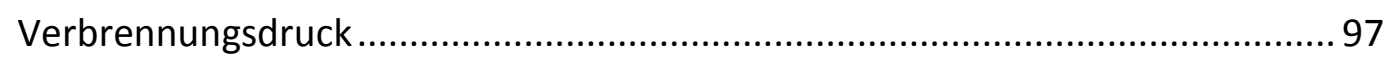

Tabelle 5.1: Übersicht zur Validierung bekannter VVPe auf Komponentenebene................. 115 


\section{Formelzeichen}

\section{Zeichen Einheit Bedeutung}

\begin{tabular}{|c|c|c|}
\hline$A$ & {$\left[\mathrm{~mm}^{2}\right]$} & nominelle Kontaktfläche \\
\hline$A_{l, 0 h}$ & {$\left[\mathrm{~mm}^{2}\right]$} & nichtverschlissene Fläche \\
\hline$A_{l, x h}$ & {$\left[\mathrm{~mm}^{2}\right]$} & verschlissene Fläche nach $\mathrm{x}$-Stunden \\
\hline$A_{R}$ & {$\left[\mathrm{~mm}^{2}\right]$} & reale Kontaktfläche \\
\hline$A_{T}$ & {$\left[\mathrm{~mm}^{2}\right]$} & Tellerplanfläche \\
\hline$A_{v}$ & {$\left[\mathrm{~mm}^{2}\right]$} & Ventilquerschnitt \\
\hline$A_{W, x h}$ & {$\left[\mathrm{~mm}^{2}\right]$} & Verschleißfläche \\
\hline $\mathrm{C}_{\mathrm{m}}$ & {$[\mathrm{m} / \mathrm{s}]$} & mittlere Kolbengeschwindigkeit \\
\hline$d_{T}$ & {$[\mathrm{~mm}]$} & Ventiltellerdurchmesser \\
\hline$d_{s}$ & {$[\mathrm{~mm}]$} & Ventilschaftdurchmesser \\
\hline $\mathrm{D}$ & {$[\mathrm{mm}]$} & Zylinderdurchmesser \\
\hline $\mathrm{e}$ & {$[1]$} & Stoßzahl \\
\hline$E$ & {$[\mathrm{GPa}]$} & Elastizitätsmodul \\
\hline$E^{\prime}$ & {$[\mathrm{GPa}]$} & angepasster Elastizitätsmodul \\
\hline $\mathrm{F}_{\mathrm{N}}$ & {$[N]$} & Normalkraft \\
\hline $\mathrm{F}_{\mathrm{p}}$ & {$[N]$} & Prüfkraft \\
\hline$F_{R}$ & {$[N]$} & Reibkraft \\
\hline $\mathrm{F}_{\text {Schlag }}$ & {$[N]$} & Aufschlagskraft \\
\hline $\mathrm{F}_{\mathrm{T}}$ & {$[N]$} & Tangentialkraft \\
\hline $\mathrm{h}$ & {$[\mathrm{mm}]$} & Ventilhub \\
\hline $\mathrm{h}_{\mathrm{CR}}$ & {$[\mathrm{mm}]$} & kritische Eindringtiefe \\
\hline $\mathrm{h}_{\max }$ & {$[\mathrm{mm}]$} & max. Ventilhub \\
\hline $\mathrm{H}$ & {$[\mathrm{HV}]$} & Härte \\
\hline$k_{\mathrm{ad}}$ & {$[1]$} & Wahrscheinlichkeitsfaktor von Verschleißpartikeln \\
\hline $\mathrm{m}$ & {$[\mathrm{kg}]$} & Masse \\
\hline $\mathrm{n}$ & {$[1]$} & Anzahl \\
\hline $\mathrm{p}$ & {$[\mathrm{N} \mathrm{s}]$} & Impuls \\
\hline $\mathrm{p}_{\mathrm{m}}$ & {$[\mathrm{MPa}]$} & mittlerer Kontaktdruck \\
\hline $\mathrm{p}_{z}$ & [bar] & Zünddruck \\
\hline r & {$[\mathrm{mm}]$} & Radius \\
\hline $\mathrm{ra}_{\mathrm{a}}$ & {$[\mathrm{mm}]$} & äußerer Radius \\
\hline$r_{i}$ & {$[\mathrm{~mm}]$} & innerer Radius \\
\hline s & {$[\mathrm{mm}]$} & Gleitweg \\
\hline$t_{\text {Stoß }}$ & {$[\mathrm{ms}]$} & Stoßzeit \\
\hline $\mathrm{T}$ & {$\left[{ }^{\circ} \mathrm{C}\right]$} & Temperatur \\
\hline$U$ & {$[\mathrm{~mm}]$} & Umfang \\
\hline
\end{tabular}




$\begin{array}{lll}V_{R} & {[\mathrm{~mm} / \mathrm{s}]} & \text { Reibgeschwindigkeit } \\ V_{W} & {\left[\mathrm{~mm}^{3}\right]} & \text { volumetrischer Verschleiß } \\ V_{W, x h} & {\left[\mathrm{~mm}^{3}\right]} & \text { Verschleißvolumen nach x-Stunden } \\ W_{m} & {[\mathrm{~m} / \mathrm{s}]} & \text { Strömungsgeschwindigkeit am Ventilsitz } \\ W & {\left[\mathrm{~mm}^{3} / \mathrm{h}\right]} & \text { Verschleißrate } \\ \alpha & {\left[{ }^{\circ}\right]} & \text { Ventilsitzwinkel } \\ \beta & {[\mu \mathrm{m}]} & \text { angenommener Radius für alle Rauheitsspitzen } \\ \delta & {[\mu \mathrm{m}]} & \text { Oberflächenabstand } \\ \mu & {[1]} & \text { Reibkoeffizient } \\ V & {[1]} & \text { Querdehnungszahl (Poissonzahl) } \\ \sigma & {[\mu \mathrm{m}]} & \text { Standardabweichung } \\ \tau_{S} & {\left[\mathrm{~N} / \mathrm{mm}^{2}\right]} & \text { Scherfestigkeit } \\ \psi & {[1]} & \text { Plastizitätsindex } \\ \psi^{*} & {[1]} & \text { allgemeiner Plastizitätsindex }\end{array}$




\section{Abkürzungsverzeichnis}

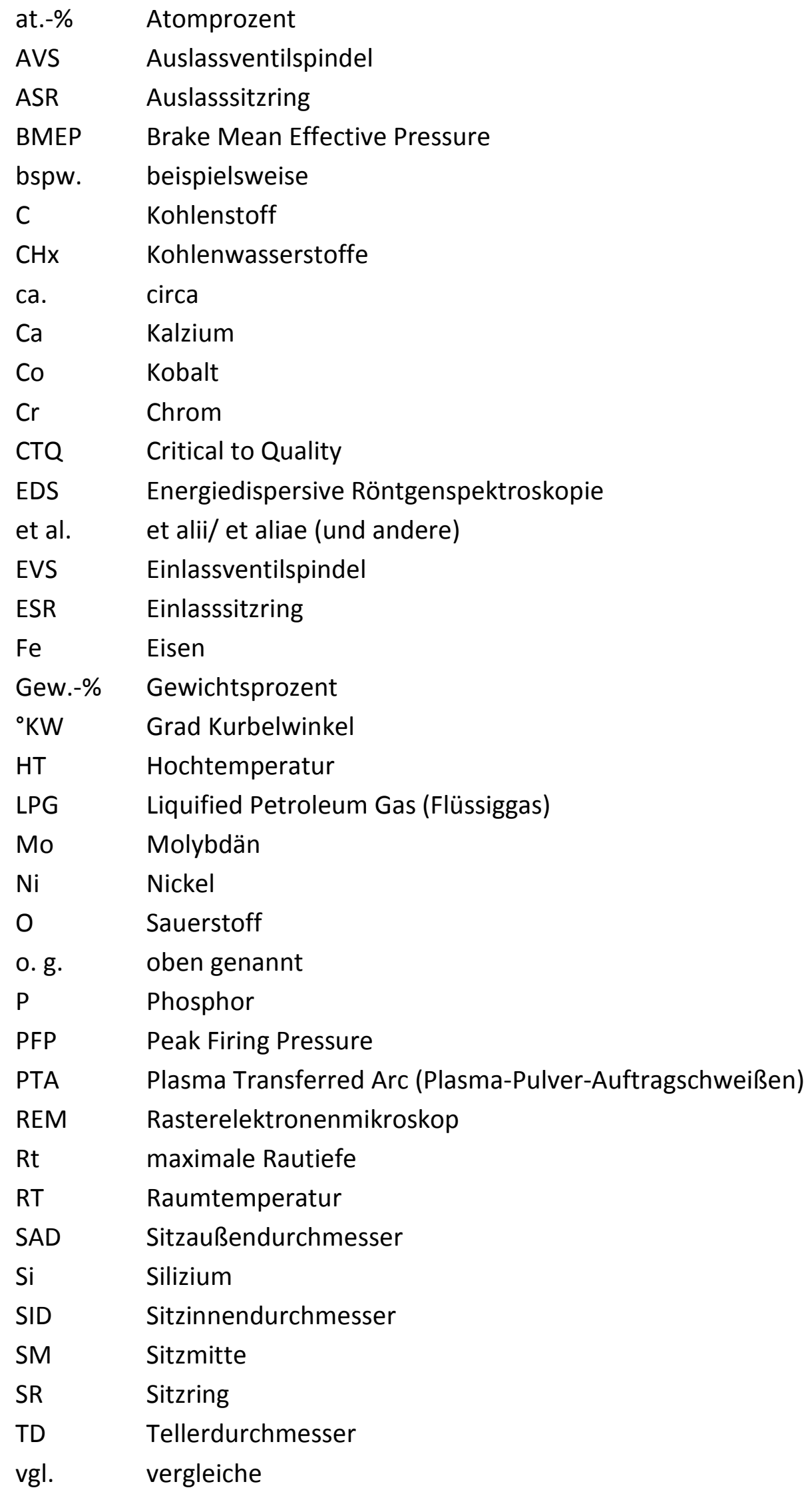




$\begin{array}{ll}\text { VS } & \text { Ventilspindel } \\ \text { VSR } & \text { Ventilsitzring } \\ \text { VV } & \text { Ventilverschleiß } \\ \text { VVP } & \text { Ventilverschleißprüfstand } \\ \text { W } & \text { Wolfram } \\ \text { z. B. } & \text { zum Beispiel } \\ \text { z. T. } & \text { zum Teil }\end{array}$




\section{$1 \quad$ Einleitung}

\subsection{Motivation des neuartigen Ventilverschleißprüfstands}

Charakteristisch für die Großmotorenentwicklung ist seit Jahrzehnten das Spannungsfeld von Effizienz und Lebensdauer. Mit dem Begriff Großmotor sind in dieser Arbeit Motoren mit einem Zylinderdurchmesser $\geq 150 \mathrm{~mm}$ gemeint. Betrachtet man die Effizienzsteigerungen seit den 1960er Jahren, so haben sich der Verbrennungsspitzendruck (PFP) von 50 auf 250 bar um den Faktor 5 und der Mitteldruck (BMEP) von 7 auf 30 bar in etwa um den Faktor 4 für Dieselmotoren erhöht [1], wobei Großmotoren in den 60er Jahren des 20. Jahrhunderts noch keine Motoren für Kraftwerke sondern vielmehr für Schiffe und Lokomotiven waren. Abbildung 1.1 veranschaulicht die Entwicklung der Verbrennungsdrücke von 1960 bis heute. Großgasmotoren erreichen gegenwärtig mechanische Wirkungsgrade von bis zu 51 \% [2].

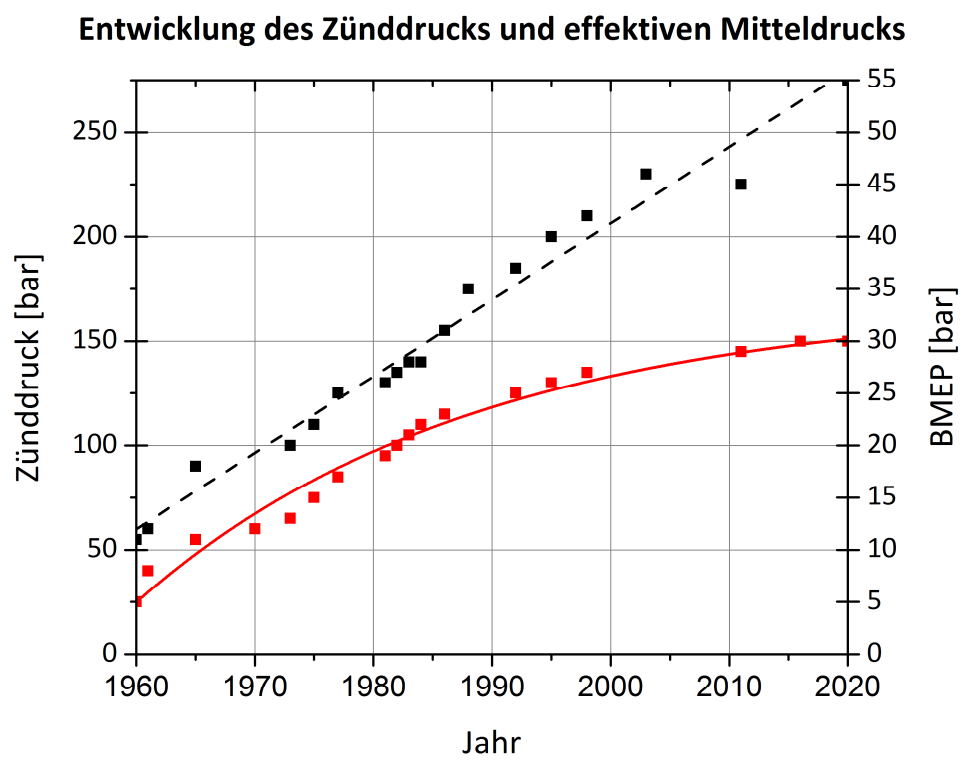

\section{Abbildung 1.1: Entwicklung der Verbrennungsspitzendrücke von Dieselmotoren von 1960 bis heute in Anlehnung an [3]}

Problemverschärfend wirken auf die Entwicklung in den letzten Jahren immer strengere Emissionsvorschriften wie z.B. IMO III, EPA Tier 4, TA-Luft 2017. In diesem Kontext bieten effiziente Großgasmotoren im Systemverbund mit neuen Verbrennungskonzepten potenzielle Entwicklungen für einen emissionsreduzierten Betrieb von stationären wie instationären Applikationen. Eine Indikation der Dieselsubstitution durch Gas ist für alle Anwendungen des Großmotors erkennbar [4], [5]. Darüber hinaus führen hybride Applikationen in Verbindung mit Windkraft- [6] und Photovoltaikanlagen [7] oder Batterien [8] zu einem neuen Betriebsverhalten für Großmotoren [9]. Ein verschärfter transienter Betrieb gekoppelt mit hundertfachen Shutdowns pro Jahr können das Ergebnis sein [10], [11]. Auf der Komponentenebene bedeuten diese zum Teil neuartigen Betriebsbedingungen erhöhte thermomechanische und chemische Belastungen. Für das in dieser Arbeit betrachtete 
tribologische System Ventilspindel (VS)/Sitzring (SR) in Großgasmotoren resultiert ein neues Belastungskollektiv. Die bekannten Arbeiten zum Tribosystem VS/SR verdeutlichen die Wichtigkeit von Verbrennungsrückständen zur Bildung verschleißminimierender Triboschichten auf den im Kontakt stehenden Oberflächen [12]. Verschärfte Emissionsvorgaben insbesondere im Hinblick auf Rußemissionen führen hingegen zu einer Reduktion von Rückständen. Ein höherer Verschleiß des Tribosystems VS/SR kann die Folge sein, wie die Untersuchungen später im Kapitel 2.3 mit Verschleißraten von bis zu $11,8 \mathrm{~mm}^{3} / \mathrm{h}$ verdeutlichen. Abbildung 1.2 veranschaulicht ausgewählte VSn mit Hochverschleiß aus hochbelasteten magerverbrennenden Großgasmotoren.
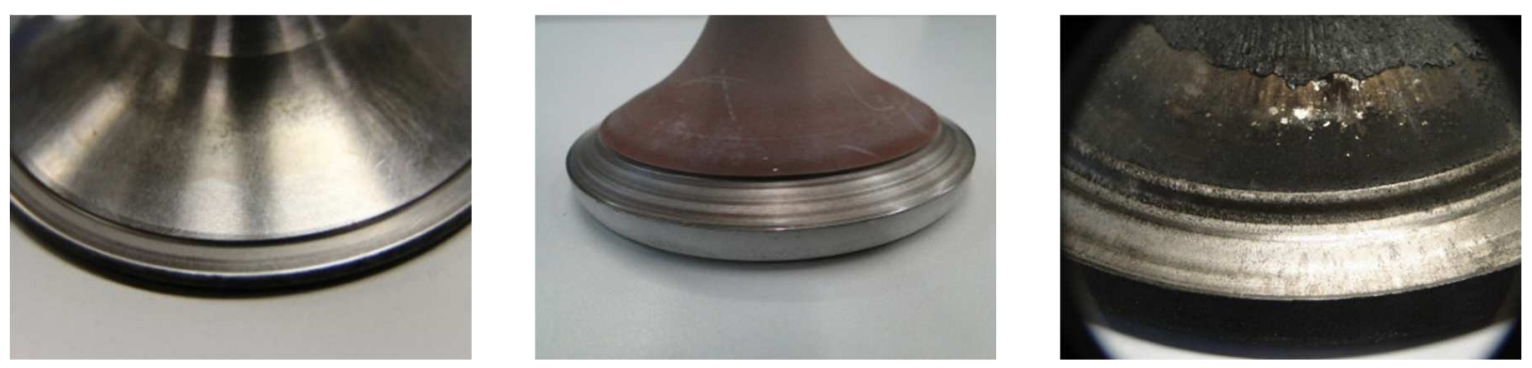

Abbildung 1.2: Verschlissene VSn aus hochbelasteten Großgasmotoren

Vor dem Hintergrund neuartiger und steigender Belastungskollektive erreichen die vorhandenen konstruktiven und technologischen Ansätze ihre Grenzen. Das heutige Verständnis über die eigenschaftsbestimmenden Mechanismen zum Ventilverschleiß für hocheffiziente Großgasmotoren ist für eine nachhaltige Problemlösung nicht ausreichend. Es besteht ein deutlicher Forschungsbedarf. Die vorliegende Dissertation behandelt einen neuartigen Ventilverschleißprüfstand (VVP) für Großgasmotoren zur erstmalig getrennten Untersuchung der beiden Phasen Ventilschließen und Verbrennungsdruck auf den Ventilverschleiß. Sie teilt sich in drei Abschnitte auf:

Im ersten Abschnitt werden die mikrostrukturellen Veränderungen in der tribologisch beanspruchten Randschicht von verschlissenen VSn in Großgasmotoren aus dem Feld zur Identifikation der eigenschaftsbestimmenden Verschleißmechanismen betrachtet. Aus den Untersuchungsergebnissen werden die Anforderungen für den VVP abgeleitet.

Im zweiten Abschnitt wird die Entwicklung, Konstruktion und der Aufbau des neuen VVP vorgestellt. Hierbei wird beginnend mit dem Prüfstandskonzept über die Wahl der Aktuatorik und des Maschinengestells auf die Konstruktion einer gasdichten Klimakammer und induktiven Heizung sowie dem Kühlsystem bis zur Validierung eingegangen.

Im dritten Abschnitt sollen erste Ergebnisse aus zwei Versuchsreihen zur experimentellen Simulation von Ventilschließen und Verbrennungsdruck bei $380^{\circ} \mathrm{C}$ an Luft zusammen mit mikrostrukturellen Untersuchungen und neue Erkenntnissen vorgestellt werden.

Die experimentellen Untersuchungen sollen in Verbindung mit der Mikrostrukturanalyse zu einem besseren Verständnis der eigenschaftsbestimmenden Verschleißmechanismen unter Ventilschließen und Verbrennungsdruck beitragen. Aus den Erkenntnissen können neue Lösungsansätze für einen reduzierten Verschleiß des Tribosystems VS/SR in Großgasmotoren entwickelt werden. 


\subsection{Tribologische Grundlagen}

Reibung und Verschleiß sind Systemeigenschaften, hingegen sind Härte und Festigkeit Werkstoffeigenschaften [13], [14]. Aussagen über das Verschleißverhalten von Werkstoffen sind immer nur für ein bestimmtes tribologisches System (Tribosystem) möglich. Für das Verständnis und die Einordnung der in dieser Arbeit präsentierten Ergebnisse werden in diesem Kapitel der Stand der Technik und der Wissenschaft zu den tribologischen Grundlagen im Hinblick auf das Phänomen Ventilverschleiß zusammengefasst. Behandelt werden daher die Verschleißarten Gleit- und Stoßverschleiß im ungeschmierten Kontakt metallischer Werkstoffe mit Schwerpunkt auf metalloxidische Triboschichten, ohne dabei näher auf die Verschleißarten Wälz-, Schwingungs-, Furchungs-, Strahl- und Erosionsverschleiß einzugehen. Die Verwendung von Begriffen und Definitionen versteht sich im Sinne von [15] und [16]. Für eine ausführliche Abhandlung der Grundlagen wird [14], [16], [17] und [18] empfohlen.

Der mit dem Jost-Report [19] im Jahr 1966 eingeführte Terminus Tribologie [20] leitet sich aus

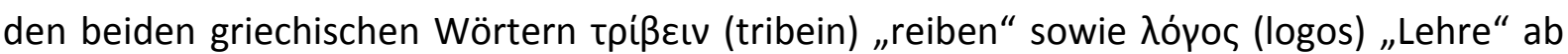
und bedeutet ursprünglich Reibungslehre [21]. Nach [15] wird die Tribologie als "die Wissenschaft und Technik von aufeinander einwirkenden Oberflächen in Relativbewegung" definiert und beinhaltet die Gebiete Reibung, Verschleiß sowie Schmierung. Die Ursprünge der Tribologie lassen sich bereits vor über 10.000 Jahren mit der Nutzung von Feuersteinen finden [21]. Erste belegte wissenschaftliche Arbeiten zum Thema Reibung gehen auf Leonardo da Vinci (1452-1519) zurück, er unterschied zwischen Gleit- und Rollreibung und erkannte, dass die Reibkraft abhängig von der Normalkraft aber unabhängig von der Kontaktfläche ist [22]. Die ökonomische Bedeutung der Tribologie und der damit verbundene Forschungsbedarf wurden in mehreren Studien belegt [19], [23], [24], [25] und [26]. Abbildung 1.3 veranschaulicht schematisch ein Tribosystem vereinfachend aus Grundkörper, Gegenkörper, Zwischenstoff und Umgebungsmedium.

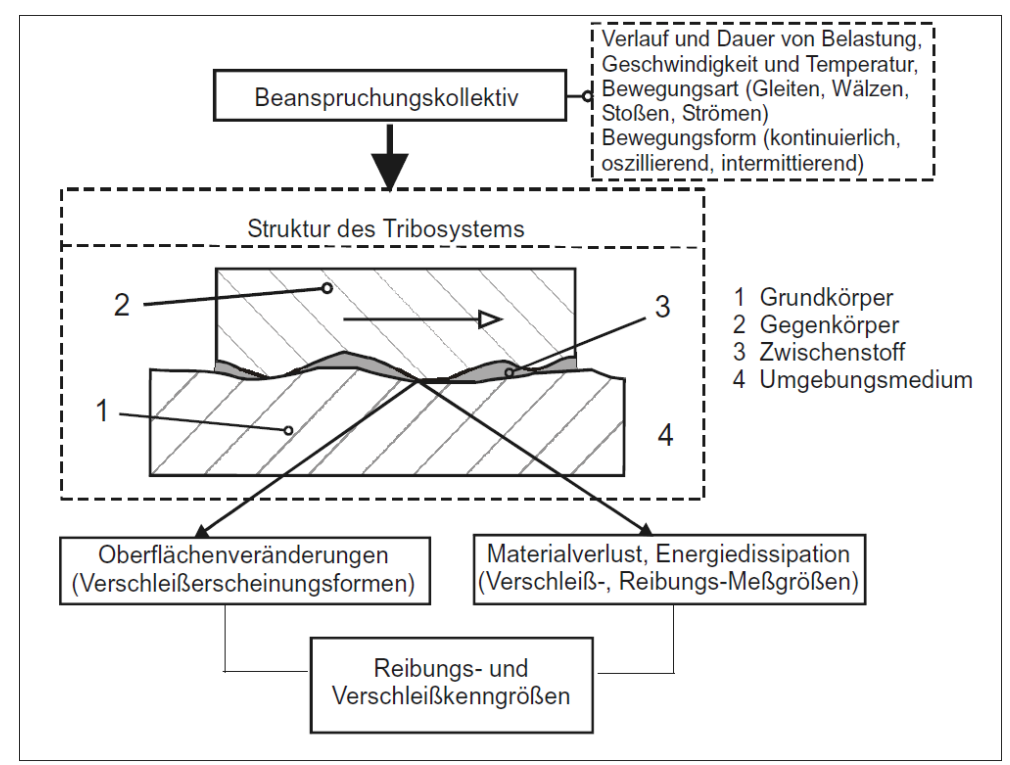

Abbildung 1.3: Schematische Darstellung eines tribologischen Systems nach [15] 
Die Gesamtheit aller Einflussgrößen wirkt als Belastungskollektiv auf ein Tribosystem. Signifikante Einflussgrößen können sein: Normalkraft $F_{N}$, Tangentialkraft $F_{T}$, Reibgeschwindigkeit $V_{R}$, Temperatur $T$, Verlauf von Dauer und Belastung, Bewegungsart und Bewegungsform. Darüber hinaus sind weitere Parameter wie Atmosphäre, Vibrationen, Rauheit, Verunreinigungen u. a. zu betrachten. Als Systemantwort können Kennwerte für die Reibung (Energiedissipation) und den Verschleiß (Werkstoffverlust, Oberflächenveränderungen) auftreten. Wie die Arbeiten von [27] verdeutlichen, können kleine Änderungen des Beanspruchungskollektivs deutlich veränderte Verschleißraten zur Folge haben. Bei der Betrachtung möglicher Verschleißverhalten von Tribosystemen können diese meist als nichtlinear, mit linearem Verhalten unter bestimmten Bedingungen und einer begrenzten Parameteranzahl, angesehen werden [28]. Tribosysteme erfüllen in der Technik vielfältige Funktionen [14]. Im Bereich der Ingenieurwissenschaften finden sie sich bspw. in der Energieübertragung (Kolben/Zylinder), im Umformen (Schmiedewerkzeuge) oder der Stoffabdichtung (VS/SR). Für die Entwicklung und Konstruktion eines neuartigen VVPs behandelt diese Arbeit das Verschleißphänomen Ventilverschleiß in Großgasmotoren. Reibung und Verschleiß im ungeschmierten Gleitkontakt sind Phänomene des Tribosystems VS/SR, weshalb in den folgenden Kapiteln auf diese beiden Größen näher eingegangen werden soll.

\subsubsection{Gleitreibung}

Reibung ist ein Phänomen von sich berührenden Oberflächen und kann nach Blau [29] als Widerstand definiert werden, der einer Relativbewegung zweier aufeinander gleitender Körper entgegenwirkt. Der Reibkontakt des Tribosystems VS/SR ist durch das Zusammenpressen von zwei ebenen Festkörpern gekennzeichnet. Daher wird im Folgenden näher auf diese Art des Oberflächenkontakts eingegangen.

Nach Stribeck [30] werden unterschiedliche Reibungszustände unterschieden. Relevant für das Tribosystem VS/SR sind die Zustände Festkörper- und Grenzflächenreibung (metalloxidischer Schutzfilm). Der Reibkontakt von metallisch reinen Oberflächen ist der reinen Festkörperreibung zu zuordnen. Der Kontakt erfolgt nach [31] direkt zwischen den Festkörpern. Da Vinci, Amontons, Desaguliers und Coulomb leisteten erste belegte Arbeiten zur Festkörperreibung von ebenen Oberflächen. Guillaume Amontons (1663-1705) erkannte die Rauheit als Ursache von Reibung und nahm an, dass ein mechanisches Verzahnen die Reibungskraft erklärt. Im nächsten Schritt entwickelte Theophilius Desaguliers (1683-1744) ein erstes Reibungsmodell auf Basis von Adhäsion und Kohäsion. Charles Augustin de Coulomb (1736-1806) setzte später auf den Arbeiten von Amontons auf und formulierte die These, wonach der Reibkoeffizient einer Fläche nicht von der Belastung abhängt. Leonhard Euler (1707-1783) führte anhand von Untersuchungen an der schiefen Ebene den Reibkoeffizienten ${ } \mu$ “ ein. Im Ergebnis hielt man fest, die Reibungskraft $F_{R}$ ist proportional zur Normalkraft $F_{N}$. Das Verhältnis nannte man Reibungszahl $\mu$. 


$$
\mu=\frac{F_{R}}{F_{N}}
$$

Mit der Erzeugung des technischen Vakuums war die Möglichkeit geschaffen, die reine Festkörperreibung zu untersuchen, da die Bildung von Adsorptions-, Oxid- oder Reaktionsschichten unterbunden werden konnte [32]. Die Arbeiten von Bowden und Tabor korrigierten die These von Coloumb, wonach bei kleinen Rauheiten eine Interaktion zwischen den Kontaktflächen zu beobachten ist, welche zu Mikroverschweißungen aufgrund von Adhäsion führt [33]. Nach der Bowden-Tabor Theorie hängt die Bildung der Mikroverschweißungen von der Scherfestigkeit $\tau_{S}$ des weicheren Reibpartners und dem mittleren Kontaktdruck $\mathrm{p}_{\mathrm{m}}$ ab, wobei der Druck die reale Kontaktfläche $A_{R}$ bestimmt.

$$
\begin{gathered}
F_{R}=\tau_{S} \times A_{R} \\
F_{N}=p_{m} \times A_{R}
\end{gathered}
$$

Somit ergibt sich als neuer Ansatz für den Reibkoeffizienten $\mu$ :

$$
\mu=\frac{\tau_{S} \times A_{R}}{p_{m} \times A_{R}}=\frac{\tau_{S}}{p_{m}}
$$

Die Herausforderung der Bowden-Tabor Theorie ist die Ermittlung der realen Kontaktfläche $A_{R}$. Theoretisch ist diese Fläche die Summe aller Mikrokontakte.

$$
A_{R}=\sum_{i=1}^{n} A_{R, n}
$$

Aus den Arbeiten von [34] ist bekannt, dass die reale Kontaktfläche $A_{R}$ deutlich kleiner als die nominelle Kontaktfläche ist, wobei sich die reale Kontaktfläche $A_{R}$ im Gleitzustand im Vergleich zum statischen Zustand vergrößert (Abbildung 1.4). Man nimmt nach [35] ein Verhältnis von ca. 1:10.000 an. 

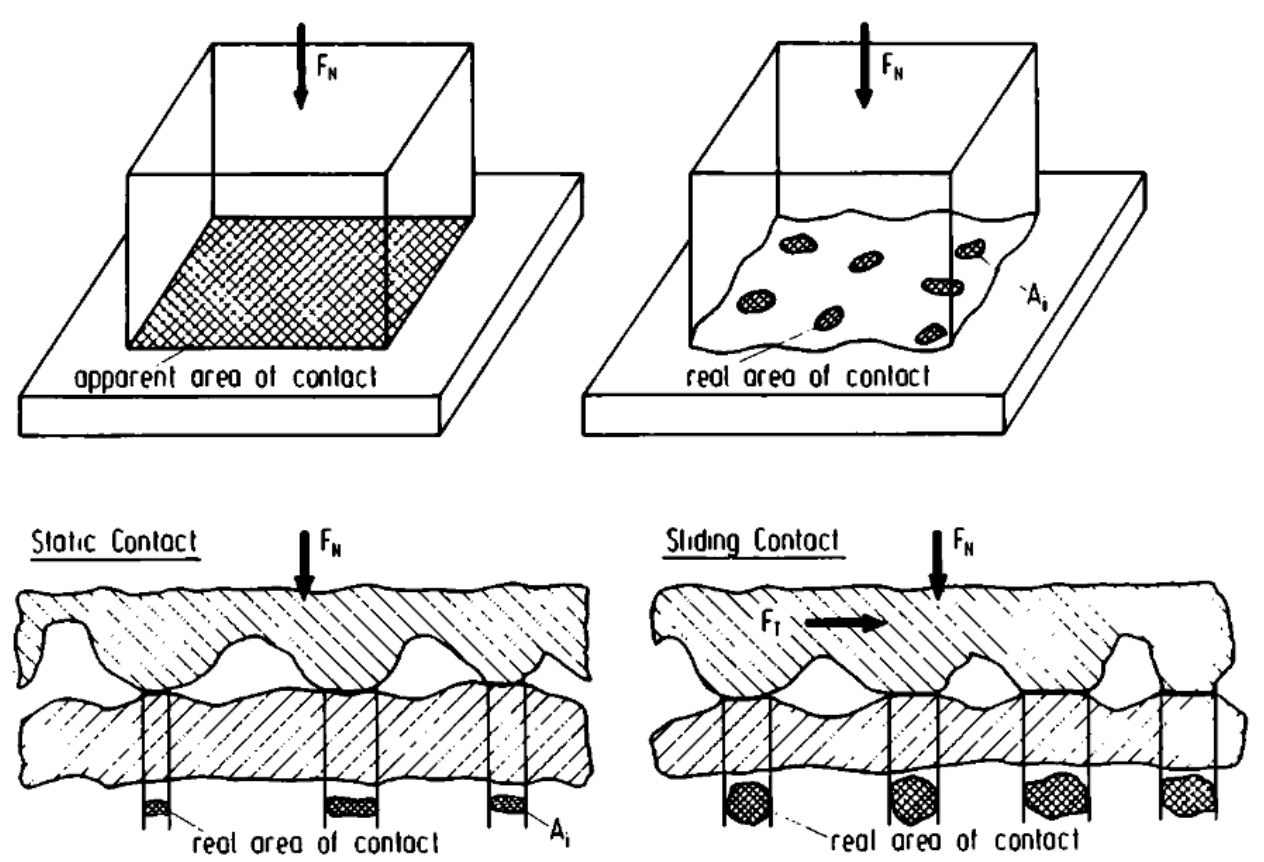

\section{Abbildung 1.4: Nominelle und reale Kontaktfläche nach [22]}

Aufsetzend auf den Arbeiten von Holm [36] führten Greenwood und Williamson 1966 unter der Annahme, dass alle Rauheitsspitzen von ebenen Oberflächen kugelförmig, die Spitzen den gleichen Radius und die Höhe der Rauheitsspitzen zufällig sind, den Plastizitätsindex $\psi$ zur Beschreibung des Übergangs vom elastischen zum plastischen Kontakt ein [37]. Die Frage, welche Rauheitsspitzen elastisch oder plastisch im Moment des Reibkontakts deformiert werden, konnte nun besser abgeschätzt werden. Er berechnet sich aus den standardisierten Variablen eines angepassten E-Modul E', der Härte $H$ des weicheren Werkstoffs, der Standardabweichung $\sigma$ bei Gauss'scher Verteilung aller Rauheitsspitzen und einem angenommenen Radius $\beta$ für alle Rauheitsspitzen:

$$
\psi=\frac{E^{\prime}}{H} \times \sqrt{\frac{\sigma}{\beta}}
$$

mit

$$
\frac{1}{E^{\prime}}=\frac{1-v_{1}^{2}}{E_{1}}+\frac{1-v_{2}^{2}}{E_{2}}
$$

wobei $E_{1}, E_{2}$ die E-Module und $v_{1}, v_{2}$ die Querdehnungszahlen (Poissonzahl) der beiden Festkörper sind. Der Index indiziert, ob ein Kontakt elastisch oder plastisch ist. Elastischer Kontakt liegt bei $\psi<0,6$ vor, plastische Deformation erfolgt bei $\psi>1$. Whitehouse und Archard erweiterten die These von Greenwood und Williamson später um die Erkenntnis, dass höhere Rauheitsspitzen schärfere Radien als tiefere Spitzen aufweisen [38]. Sie führten einen allgemeineren Plastizitätsindex $\psi^{*}$ ein: 


$$
\psi^{*}=0,69 \times \frac{E^{\prime}}{H} \times \frac{\sigma^{*}}{\delta}
$$

wobei $E^{\prime}$ aus (1.7) folgt, $\sigma^{*}$ der Oberflächen-RMS-Wert und $\delta$ der Oberflächenabstand ist. Tabor [39] formulierte für den Kontakt einer starren Kugel und eines ebenen Festkörpers im elastisch-elastisch/plastischen Übergang die kritische Eindringtiefe $h_{\mathrm{CR}}$ :

$$
h_{C R}=0,89 \times R \times(H / E)^{2}
$$

mit dem Kugelradius R, Härte $\mathrm{H}$ und dem E-Modul des verformten Werkstoffs. Bei Betrachtung der Formeln (1.6), (1.8) und (1.9) bestimmen Oberflächentextur, Härte und E-Module die Deformation von Rauheitsspitzen, hingegen beeinflusst die aufgeprägte Normalkraft $F_{N}$ nicht unmittelbar den elastisch-plastischen Übergang. Im Hinblick auf zukünftig steigende Verbrennungsdrücke und damit einhergehend höheren Flächenpressungen zwischen VS und SR sind die Untersuchungen zu den Verschleißmechamismen im Kapitel 2.3 zu bewerten. Aus weiteren Arbeiten wurde die These formuliert, dass die Reibkraft $F_{N}$ bei statischem Kontakt für ideal elastisch-plastische Werkstoffe in erster Näherung proportional zur realen Kontaktfläche $A_{R}$ ist [40]:

$$
A_{R}=\frac{F_{N}}{p_{S}}
$$

wobei $F_{N}$ die Normalkraft auf die Kontaktflächen und $p_{s}$ die Fließspannung des weicheren Werkstoffs sind. Holm führte die Idee ein, dass neben globalen elastischen Spannungen lokal plastische Kontaktsituationen auftreten können [36]. Archard schärfte die Proportionalität von Fläche und aufgeprägter Last durch die Annahme, dass ein Lastanstieg neue Kontaktflächen bzw. eine Vergrößerung der bestehenden Flächen bewirkt. Bleibt die Anzahl der Kontakte gleich, so folgt:

$$
A_{R} \propto F_{N}^{\frac{2}{3}}
$$

Die reale Kontaktfläche $A_{R}$ für ebene Flächen berechnet sich nach [37] mit:

$$
A_{R} \propto F_{N}^{m}
$$

Zusammenfassend darf festgestellt werden, dass reale Kontaktflächen und selbst polierte Oberflächen Rauheitsspitzen und Täler aufweisen. Im Kontakt berühren sich nicht alle Rauheitsspitzen und es kommt, wie die Theorien zur realen Kontaktfläche verdeutlichen, zu einer unbestimmt großen Kontaktfläche zwischen Grund- und Gegenkörper mit elastischplastischen Anteilen, wobei die reale Kontaktfläche näherungsweise proportional zur 
aufgeprägten Normalkraft ist. Reibung findet mit der Bewegungshemmung und Energiedissipation in der realen Kontaktfläche statt. Nach [22] können plastische Verformungen, Mikroverschweißungen, Aufbrechen der lokalen Mikroverschweißungen, Verzahnung der Rauheitsspitzen und Dämpfungsprozesse beobachtet werden, wobei die auftretende Reibungsenergie zu ca. $90 \%$ in Wärme und somit in höhere Oberflächentemperaturen umgewandelt wird [41]. Der Rest verursacht Schallwellen, erzeugt neue Oberflächen und Materialdefekte. Reibungsinduzierte Defekte können bspw. Versetzungen, Verfestigungen oder Risse sein, die in der Folge zu Verschleiß führen können. Diese Werkstoffschädigungen werden später von Bedeutung sein.

\subsubsection{Verschleiß}

Volumenbeanspruchungen resultieren aus eingeleiteten Lasten in Form von Spannungen und Verformungen [13]. Bei Versagen eines Bauteils kommt es zur Rissbildung und später zum Bruch. Hingegen sind die Folgen von Oberflächenbeanspruchungen Korrosion oder Verschleiß. Beide Beanspruchungsarten gefährden die technische Funktion von Bauteilen. Die Tribologie befasst sich mit Oberflächenbeanspruchungen infolge von mechanischen, thermischen und chemischen Belastungen. Für das Verständnis von tribologischen Vorgängen sind möglichst alle Einflussparameter und Werkstoffe zu betrachten, da eine tribologische Beanspruchung „komplexe und irreversible Prozesse in der Grenzschicht“ [16] verursachen kann. Den im Kontakt stehenden Oberflächen werden Normal- und Tangentialkräfte aufgeprägt. Die Systemeigenschaft Verschleiß definiert sich nach [15] als „fortschreitender Werkstoffverlust aus der Oberfläche eines festen Körpers, hervorgerufen durch mechanische Ursachen, d.h. Kontakt und Relativbewegung eines festen, flüssigen oder gasförmigen Gegenkörpers. ", wobei Energieumsetzungsprozesse in der Grenzschicht stattfinden [16]. Es gilt, Ursache von Verschleiß ist eine tribologische Beanspruchung. In der Folge treten physikalische und chemische Prozesse an den Oberflächen von Grund- und Gegenkörper auf. Die Summe aller Prozesse charakterisiert die eigenschaftsbestimmenden Verschleißmechanismen [14], wobei in den meisten Anwendungsfällen eine Überlagerung der Mechanismen zu beobachten ist [15]. Basierend auf den Arbeiten von Burwell [42] werden heute die vier grundlegenden Verschleißmechanismen Adhäsion, Abrasion, Oberflächenzerrüttung und tribochemische Reaktion bei Festkörpern unterschieden [14], [15]. Da alle vier Mechanismen bei dem Tribosystem VS/SR beobachtet wurden [43], werden diese folgend kurz beschrieben.

Der mechanisch-dominierte Verschleißmechanismus Oberflächenzerrüttung ist durch eine zyklische Beanspruchung von Festkörperoberflächen charakterisiert. Die zyklisch auftretenden Belastungen bewirken eine Akkumulation von plastischen Dehnungen in der tribologisch beanspruchten Randschicht und führen nach einer Inkubationsphase mit mildem Verschleiß zu einer Werkstoffermüdung. Beginnend mit einer Rissinitierung und anschließender Risspropagation kommt es zur Bildung von Verschleißpartikeln. Neben einer Stoßbelastung führt auch Gleitreibung zur Oberflächenermüdung. In diesem Fall ist eine Rissbildung in der plastifizierten Randschicht parallel zur Verschleißfläche zu beobachten. In der Folge können plättchenförmige Verschleißpartikel herausbrechen. Diese 
Verschleißerscheinungsform wird als Delamination bezeichnet [44]. Grübchen und Risse sind charakteristische Erscheinungsformen der Oberflächenzerrüttung.

Der zweite mechanisch-dominierte Verschleißmechanismus ist die Abrasion, die durch ein Furchen und Ritzen der Kontaktflächen infolge eines härteren Gegenkörpers als der tribologisch beanspruchte Grundkörper gekennzeichnet ist. Nach [22] werden die vier Untermechanismen Mikropflügen, Mikroermüden, Mikrozerspanen und Mikrobrechen unterschieden. Das ideale Mikropflügen führt zu keinem messbaren Werkstoffabtrag. Der Werkstoff wird plastisch verformt und vom Abrasiv zu den Furchungsrändern gedrückt. Beim Mikroermüden bewirkt ein zyklisches Mikropflügen eine Akkumulation von plastischen Dehnungen und führt zu einer lokalen Ermüdung. Ein Werkstoffabtrag kann messbar werden. Der ideale Mechanismus des Mikrospanens ist durch die Bildung eines Mikrospans, welcher gleich dem Volumen der Furche ist, vor dem Abrasiv gekennzeichnet. Das Mikrobrechen infolge einer Rissbildung und Rissausbreitung in Richtung der Verschleißfurche ist besonders bei spröden Werkstoffen typisch.

Bei dem Verschleißmechanismus Adhäsion werden die kontaktmechanischen Belastungen um stoffliche Wechselwirkungen erweitert. Eine Eigenschaft von technischen Oberflächen ist die Rauheit. Somit erfolgt die Übertragung von Kräften lokal an einzelnen Mikrokontakten. Elastisch-plastische Deformationen in diesen Kontaktstellen infolge von Normal- und Scherkräften bewirken eine Zerstörung der oberflächlichen Adsorptions- und Reaktionsschichten (Abbildung 1.7). Die entstehenden metallisch blanken Oberflächen können im Kontakt mit einem Gegenkörper mehr oder weniger feste Verbindungen durch atomare Bindungen eingehen. Dieses Phänomen der lokalen Mikroverschweißungen wird als Adhäsion bezeichnet. Adhäsion ist charakteristisch für den ungeschmierten Reibkontakt und besonders im Vakuum zu beobachten. Ob die Trennung von Grund- und Gegenkörper inneroder außerhalb der Bindungsebene erfolgt, hängt von der Festigkeit der Mikroverschweißungen ab. Ein Lösen der Verbindung außerhalb der Bindungsebene resultiert in einer Werkstoffübertragung insbesondere nach mehrfachen Übergleitungen. Eine Hin- und Rückübertragung von Werkstoff ist möglich. Als Ergebnis aus Adhäsionsexperimenten ist bekannt, dass bei Metallen die Adhäsion mit steigender Härte abnimmt [45] und im MetallMetall-Kontakt eine Werkstoffübertragung vom weicheren zum härteren Werkstoff erfolgt [46]. Adhäsive Verschleißerscheinungen können plastische Deformationen, Scherwaben, Riefen, Gefügeumwandlungen, Werkstoffübertragung und oberflächliche Schubrisse sein. Zur Verschleißberechnung hat Archard 1956 in [47] ein empirisches Modell zwischen dem volumetrischen Verschleiß $V_{W}$, der Normalkraft $F_{N}$, dem Gleitweg $s$, der Härte H des weicheren Reibpartners und dem Faktor $\mathrm{k}_{\mathrm{ad}}$ als Wahrscheinlichkeit der Entstehung von Verschleißpartikeln eingeführt.

$$
V_{W}=k_{a d} \times \frac{F_{N} \times s}{H}
$$

Im metallischen Kontakt kann eine Minimierung des Gleitverschleißes durch die bevorzugte Verwendung von Werkstoffen mit kubisch-raumzentrierter oder hexagonaler Struktur 
insbesondere bei Vermeidung austenitischer Stähle erzielt werden [13]. Dieser Ansatz ist für das Tribosystem VS/SR nur bedingt realisierbar, da austenitische Ventilstähle wie $1.4882 \mathrm{im}$ Vergleich zu martensitischen Ventilstählen wie 1.4718 eine höhere Warmfestigkeit aufweisen und somit in überwiegender Zahl als Werkstoff für AVSn in Großgasmotoren eingesetzt werden. Neben der Gitterstruktur stellt die Bildung von Triboschichten eine weitere Möglichkeit zur Reduktion der Adhäsion dar.

Bei dem Verschleißmechanismus tribochemische Reaktion aktiviert eine tribologische Beanspruchung chemische Prozesse zwischen Grund- und Gegenkörper sowie angrenzenden Medien, die zur Bildung von Reaktionsschichten bzw. einzelnen Reaktionspartikeln führt [15]. Nach [48] führen mechanische und thermische Aktivierungen zu einer erhöhten Reaktionsgeschwindigkeit im Kontaktbereich. Plastische Verformungen erhöhen im Vergleich zu elastischen Verformungen im Reibkontakt die Oxidation [49]. Die Reaktionsgeschwindigkeit hängt nach [50] u. a. von der Temperaturerhöhung infolge der Reibungswärme ab. Reaktionsschichten weisen meist eine Doppelnatur auf. Oxidschichten können einerseits als Abrasiv verschleißerhöhend, andererseits, wenn die Bindungsfestigkeit zu Grund- oder Gegenkörper ausreichend groß ist, verschleißminimierend wirken [51]. Insbesondere im metallischen Kontakt kann durch eine reduzierte Adhäsion schwerem Verschleiß entgegengewirkt werden [13]. Neben dem Auftreten von Reaktionsschichten selbst, ist nach [41] die Schichtdicke verschleißrelevant. Der meist spröde Charakter nimmt mit der Schichtdicke zu, sodass es bei einer kritischen Schichtdicke zum Abplatzen kommen kann.

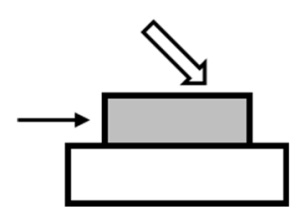

Gleiten

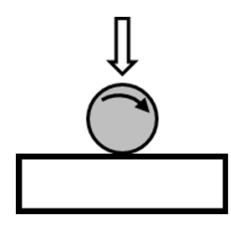

Rollen

Wälzen

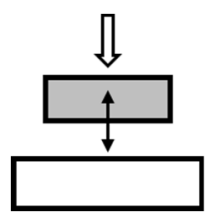

Stoßen

Prallen

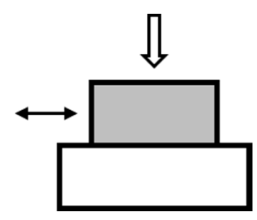

Oszillieren

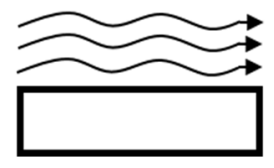

Strömen

\section{Abbildung 1.5: Schematische Darstellung der tribologischen Beanspruchung nach [15]}

Die Unterscheidung der Verschleißvorgänge in Bezug auf die bestimmende Relativbewegung führt auf die Verschleißarten Gleit-, Wälz-, Stoß-, Schwingungs-, Furchungs-, Strahl- und Erosionsverschleiß.

Die beiden Verschleißarten Gleit- und Stoßverschleiß im ungeschmierten Kontakt metallischer Werkstoffe charakterisieren das Tribosystem VS/SR; Gleitverschleiß unter Verbrennungsdruck [52], Stoßverschleiß unter Ventilschließen [43]. Daher sollen diese beiden Verschleißarten in den folgenden Kapiteln näher vorgestellt werden. Eine Betrachtung der Verschleißart Abrasion erscheint nach Rigney [53] nicht erforderlich, da der Spananteil im Vergleich zu den anderen Verschleißpartikeln klein bis nicht nachweisbar ist. 


\subsubsection{Gleitverschleiß}

Gleitverschleiß kann als Relativbewegung zwischen zwei im Kontakt stehenden ebenen festen Oberflächen unter Lasteinleitung betrachtet werden, wobei die Oberflächenschädigung nicht durch tiefe Oberflächenriefen aufgrund eindrückender Asperiten oder Fremdpartikel erfolgt [22]. Die Kontaktart, elastisch oder plastisch, ist eine Funktion von Scherbelastung, Kontaktfläche und technologischen Werkstoffeigenschaften. Im Gleitkontakt kann Verschleiß durch die vier Hauptmechanismen Oberflächenzerrüttung, Abrasion, Adhäsion und tribochemische Reaktion bewirkt werden. Inwieweit die Mechanismen anteilig wirken, hängt vom jeweiligen Belastungskollektiv und der Dauer der tribologischen Belastung ab. In der Literatur finden sich zahlreiche Arbeiten zum Gleitverschleiß, welche nur bedingt vergleichbar sind. Denn wie erwähnt, ist Verschleiß eine Systemeigenschaft. Kleine Änderungen im Belastungskollektiv können in deutlich unterschiedlichen Verschleißraten resultieren. Abbildung 1.6 veranschaulicht schematisch einen typischen Verschleißverlauf über die Zeit bzw. den Gleitweg [22]. Man unterscheidet drei Bereiche. Bereich I kennzeichnet nach [54] die Einlaufphase (Running-In) mit einem degressivem Kurvenverlauf der Verschleißrate.

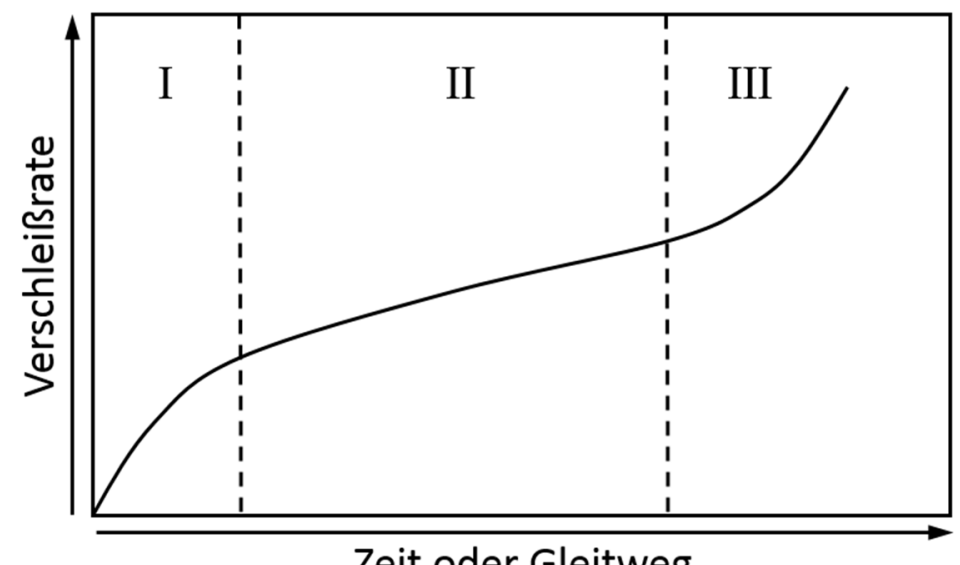

Zeit oder Gleitweg

\section{Abbildung 1.6: Schematischer Verschleißverlauf als Funktion von Belastungszeit oder Gleitweg nach [22]}

Nach [47] tritt während dieser Phase zumeist großer Verschleiß auf, wobei hohe Verschleißraten in Verbindung mit relativ großen metallischen Verschleißpartikeln typisch sind. Bereich II beschreibt einen Zustand mit nahezu konstanten Verschleißraten (SteadyState). Kleine Verschleißraten und oxidische Verschleißpartikel im Metall-Metall-Kontakt kennzeichnen diese Phase [55]. Bereich III ist charakterisiert durch einen progressiven Kurvenverlauf. Steigende Verschleißraten bestimmen zunehmend das Tribosystem und führen zur Schadensart Verschleiß mit vorzeitigem Bauteilversagen. Charakteristisch für die Verschleißart Gleitverschleiß in Bezug auf das Tribosystem VS/SR sind metalloxidische Triboschichten, welche im Folgenden näher betrachtet werden sollen. 


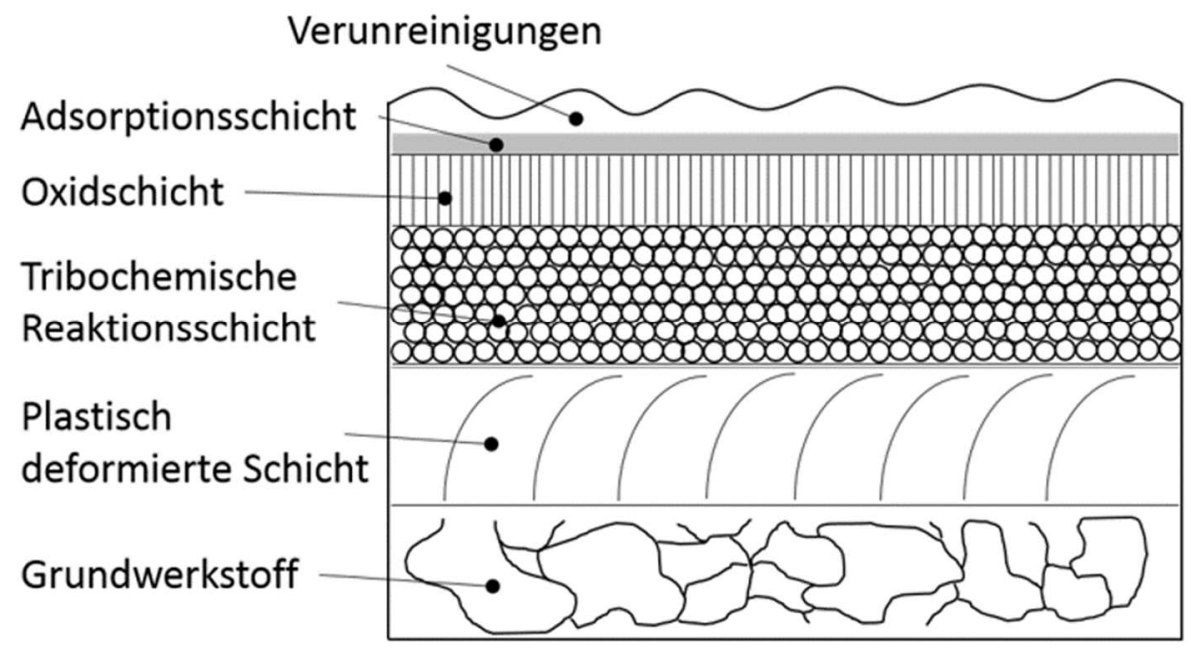

\section{Abbildung 1.7: Schematischer Aufbau technischer Werkstoffgrenzschichten und Mikrostrukturänderungen unter Gleitverschleiß nach [32]}

Am Anfang der tribologischen Belastung werden die äußeren Grenzschichten insbesondere die Oxidschicht des Metallwerkstoffs geschädigt, sodass ein Metall-Metall-Kontakt zwischen den Rauheitsspitzen von Grund- und Gegenkörper resultiert [27], [47]. In der Folge lassen sich die typischen Verschleißerscheinungen der Adhäsion wie Mikroverschweißung, plastische Deformation oder Werkstoffübertragung beobachten. Mit andauernder tribologischer Belastung kommt es zur Bildung von oxidischen Verschleißpartikeln, die entscheidend den weiteren Verschleiß bestimmen. Diese Partikel können als Festschmierstoff wirken und somit den metallischen Kontakt vermeiden [51]. Zwei Arten von Oxidationsprozessen sind bekannt, die transiente Oxidation und die Gleichgewichtsoxidation, wobei das Wachstum der Oxide von den metallischen Elementen abhängt [56]. Für den Gleitverschleiß ist die transiente Oxidation von Bedeutung [51]. Die Bildung metalloxidischer Verschleißpartikel kann nach [57] und [58] auf drei Mechanismen zurückgeführt werden. Mechanismus 1 beschreibt die Entstehung von Partikeln ausgehend von oxidierten Rauheitsspitzen. Aufgrund des reibungsbedingten Temperaturanstiegs mit Blitztemperaturen von mehreren hundert Grad Celsius [59] oder sogar bis zum Schmelzpunkt von Metallen [33] erfolgt die Bildung von Oxidschichten auf den im Kontakt stehenden Rauheitsspitzen von Grund- und Gegenkörper. Bei andauernder Gleitbelastung kann die Oxidschicht aufdicken oder delaminieren [41], somit ergibt sich wieder der Ausgangszustand eines Metall-Metall-Kontakts und der Prozess kann erneut beginnen. Mechanismus 2 ist charakterisiert durch die Oxidation von metallischen Verschleißpartikeln. Im Fall des Metall-Metall-Kontakts unter Gleitbelastung kann es zur Bildung von metallischen Partikeln im Reibspalt infolge von Mikroverschweißungen der Rauheitsspitzen oder abgescherten Asperiten kommen. Die anschließende Oxidation und Sauerstoffpenetration wird durch die größere Defektdichte der abgetragenen Partikel wie auch durch die hohen Blitztemperaturen im Reibkontakt erhöht. Mechanismus 3 betrachtet vollständig oxidierte Kontaktoberflächen. Die Oxidschichten können bei andauernder tribologischer Belastung lokal geschädigt werden. In der Folge unterbleibt der metallische Kontakt teilweise bis vollständig, sodass nach [58] und [60] ein minimierter Verschleiß beobachtet werden kann. Die Mechanismen 1 und 2 sind bereits bei Raumtemperatur 
feststellbar, hingegen wirkt Mechanismus 3 bei höheren Temperaturen infolge steigender Oxidationsneigung von Metallen. Der Einfluss oxidischer Partikel auf den Verschleiß ist zahlreich in der Literatur dokumentiert [61], [62], [63], [64] und [65]. Im Ergebnis darf festgestellt werden, der Verschleiß wird minimiert, wenn oxidische Partikel zu Triboschichten kompaktiert werden [62] und auf den Kontaktoberflächen anhaften können. Hingegen wird der Verschleiß erhöht, wenn freie oxidische Partikel als Abrasiv wirken [47]. Nach Glascott [66] wird die Verschleißentwicklung eines tribologischen Systems in Bezug auf die oxidischen Verschleißpartikel durch Beanspruchungskollektiv, Werkstoff und Härte der oxidischen Verschleißpartikel bestimmt.

Auf die Bildung verschleißminimierender Triboschichten wird nachstehend näher eingegangen. Als Ergebnis einer tribologischen Beanspruchung werden, wie im vorherigen Absatz beschrieben, metallische oder oxidische Verschleißpartikel generiert. Verbleiben die Partikel im Reibspalt, so kann ein mechanisches Vermischen der Partikel mit Grund- und Gegenkörper und oder ein Zerbrechen in kleinere Teilchen erfolgen [67]. Mit andauernder Belastung werden die Einzelpartikel aufgrund der herrschenden Flächenpressungen zwischen Grund- und Gegenkörper zu Agglomeraten kompaktiert. Im weiteren Verlauf kommt es zur Bildung lokaler Triboschichten. Mit weiter andauernder tribologischer Belastung können sich auf den Triboschichten infolge plastisch wirkender Druck- und Scherbelastungen Glazeschichten bilden [68]. Namensgebend war hierbei die auffallend glatte und glasartig glänzende Erscheinung dieser plastisch verformten Schichten [69]. Das Glazeschichten kein amorphes Gefüge aufweisen, sondern einen nanokristallinen Charakter besitzen, wurde von Stott [70] nachgewiesen. Die abweichende Chemie der Triboschichten im Vergleich zu Grundund Gegenkörper erklärt sich aus dem mechanischen Mischen der Partikel mit diesen im Reibspalt und der Oxidation. Die unterschiedliche Härte der Verschleißpartner hat einen Einfluss auf die Bildung von Triboschichten. So verdeutlichen die Arbeiten von [71] mit einem weicheren und härteren Chromstahl, dass es zu einer Akkumulation von Verschleißpartikeln in den tieferen Furchen des weicheren Werkstoffs und somit zu einer erhöhten Bildung von Triboschichten kommt. Die mikrostrukturellen und chemischen Unterschiede der Triboschichten im Vergleich zu Grund- und Gegenkörper führen zu anderen Eigenschaften. In Abhängigkeit von der chemischen Zusammensetzung kann die Härte der kompaktierten Triboschicht kleiner [12] oder größer [62] sein. Neben der Bildung von Triboschichten kann eine Gleitbelastung infolge plastischer Deformationen mikrostrukturelle Veränderungen der Randschicht von Grund- und Gegenkörper bewirken [22]. Untersuchungen von Heilmann et al. [72] an Kupfer mittels TEM verdeutlichen eine Variation der Mikrostruktur in Abhängigkeit von der Tiefe unterhalb der Kontaktfläche. Es können meist 3 typische Zonen beobachtet werden. Zone 1 als Interface ist charakterisiert durch Werkstoffübertragung aus gefeinten und gemischten Verschleißpartikeln aus Grund- und Gegenkörper. Diese Schicht entspricht der Triboschicht. Die darunterliegende Zone 2 weist häufig ein korngefeintes Gefüge auf. Eine Dehnungsakkumulation kann beobachtet werden. Daneben weist die gestreckte Morphologie der feinkörnigen Mikrostruktur in Richtung der Gleitbelastung. Mit größerem Abstand zur Oberfläche nehmen Dehnung und Defektdichte ab. Zone 3 ist durch plastische Deformationen in Form von Versetzungen jedoch ohne Kornfeinung gekennzeichnet. Die chemische 
Zusammensetzung von Zone 2 und 3 entspricht dem Ausgangswerkstoff. Auf Basis der ausgelenkten Korngrenzen kann bei unidirektionaler Gleitbelastung die Dehnung bestimmt werden. Rigney [53] hat Dehnungen von ca. $1000 \%$ in der tribologisch beanspruchten Randschicht von Kupfer nachweisen können. Weitere Gefügeerscheinungen unter Gleitreibung können zusätzlich Phasenumwandlungen oder in Werkstoffen mit harten Phasen das Brechen von Hartphasen sein [22]. Verschiedene Werkstoffpaarungen können unterschiedlich große Verschleißraten bei ähnlich großen Reibwerten aufweisen [73]. Dieses Phänomen kann durch das unterschiedliche Dissipationsvermögen der Werkstoffe erklärt werden; ein Werkstoff dissipiert mehr Energie bei der Erzeugung von Verschleißpartikeln, der andere weist eine höhere Energiedissipation durch plastische Deformation auf.

\subsubsection{Gleitverschleiß bei Hochtemperatur}

Nach Quellenlage ist der Begriff Hochtemperaturtribologie nicht klar definiert. Zur Orientierung soll nach [74] die Stabilitätsgrenze von Flüssigschmierstoffen mit $450{ }^{\circ} \mathrm{C}$ dienen. Der Temperatureinfluss auf das Verschleißverhalten eines metallischen Tribosystems ist nach [16], [18] und [22] signifikant. Mit Erhöhung der Temperatur verändern sich die mechanischen und chemischen Werkstoffeigenschaften [75]. Die mechanischen Kennwerte wie Härte, Festigkeit oder Elastizitätsmodul nehmen meist mit steigender Temperatur ab, wohingegen Duktilität, Oxidations- sowie Diffusionsprozesse von metallischen Werkstoffen mehr aktiviert werden. Im Hinblick auf die Kontaktsituation von Reibpartnern bewirkt eine steigende Duktilität eine vergrößerte reale Kontaktfläche, wodurch Adhäsionsprozesse und plastische Deformationen begünstigt werden können. Bei Veränderungen der Reibung infolge von Temperaturänderungen sind zwei Phänomene zu betrachten [18]. Phänomen 1 ist die Temperaturänderung aufgrund einer externen Wärme- oder Kühlquelle. Grund- und Gegenkörper werden im ganzen Volumen erwärmt bzw. gekühlt. Phänomen 2 beschreibt die Situation einer Temperaturerhöhung nur an den Kontaktflächen mit einem vergleichsweise kälteren Volumenwerkstoff aufgrund von Hochgeschwindigkeitsgleiten. Simon et al. konnten 1951 in [76] keinen systematischen Trend der Reibung im untersuchten Bereich von - 270 bis $330{ }^{\circ} \mathrm{C}$ feststellen. Ein weiterer Vergleich von Rabinowicz in [18] aus 50 verschiedenen Reibtests mit einem breiten Werkstoffspektrum bei verschiedenen Hochtemperaturen stützt die Erkenntnis von Simon, dass die Reibung keine temperaturabhängige Größe ist, wobei Einzeltests Veränderungen der Reibung bei steigender Temperatur aufwiesen. Fischer konnte 1988 in [77] für einen einphasigen Werkstoff kleinere Verschleißraten bei $550^{\circ} \mathrm{C} \mathrm{im} \mathrm{Vergleich}$ zu $25^{\circ} \mathrm{C}$ aufgrund erhöhter Tribooxidation messen, jedoch vergrößerte sich die Verschleißrate bei steigenden Temperaturen für mehrphasige Werkstoffe. Metalle können bei hohen Temperaturen in einem engen Temperaturbereich einen deutlichen Abfall des Reibwertes aufweisen. Peterson et al. konnten 1960 in [78] für die Paarung Nickel auf Stahl bei ca. $760^{\circ} \mathrm{C}$ die Bildung eines Oxidfilmes beobachten. In der Folge wurde kein weiterer Anstieg, sondern ein deutlicher Abfall des Reibwertes mit steigender Temperatur gemessen. Interessant hierbei ist, dass der niedrige Reibwert bei hohen Temperaturen mit eingeschalteter Kühlung bei andauernder Gleitreibung und somit reduzierter Temperatur bis ca. $400{ }^{\circ} \mathrm{C}$ "eingefroren" 
werden konnte. Eine Hysterese war zu beobachten. Außer der reinen Oxidbildung sind die Art und die Anteile der dominierenden Oxide von Bedeutung. Bei der Paarung Kobalt auf Stahl wurde im untersuchten Temperaturbereich von ca. 530 bis $760^{\circ} \mathrm{C}$ ein exponentieller Abfall des Reibwertes gemessen [18]. Die Reduzierung der Reibung oberhalb von $530{ }^{\circ} \mathrm{C}$ kann auf ein Wachstum der Oxidschicht und ebenso auf eine Änderung der Oxidart von $\mathrm{CoO} \mathrm{zu} \mathrm{Co}_{3} \mathrm{O}_{4}$ für Kobalt und $\mathrm{zu} \mathrm{Fe}_{3} \mathrm{O}_{4}$ für Stahl zurückgeführt werden. Allgemein dürfen aus den wissenschaftlichen Arbeiten der letzten Jahrzehnte zum Gleitverscheiß bei Hochtemperatur an Luft zwei allgemeine Erkenntnisse festgehalten werden. Nach [68], [71], [79], [77], [80], [81], [82], [83], [84], [85] und [86] ist eine schnelle Unterbindung des Metall-Metall-Kontakts im Vergleich zur Raumtemperatur zu beobachten. Zweitens ist ein Anstieg der Gleitreibung dann festzustellen, wenn die Schmelztemperatur von einem der Reibpartner erreicht wird. Dieses Phänomen kann aus dem Verhältnis von Oberflächenenergie und Härte erklärt werden. Die Oberflächenenergie im Zähler ändert sich unwesentlich mit steigender Temperatur, hingegen geht die Härte im Nenner mit Erreichen der Schmelztemperatur gegen Null. Wie in Kapitel 1.2.3 beschrieben, kommt es bei Raumtemperatur aufgrund der Mechanismen 1 und 2 zur Bildung oxidischer Verschleißpartikel. Verbleiben diese Partikel im Reibspalt, haben sie einen signifikanten Einfluss auf das weitere Verschleißverhalten. Bei höheren Temperaturen nimmt die Bildung von Oxiden infolge von Mechanismus 3 zu, bis die Kontaktflächen mit einer Oxidschicht überzogen sind, die sich relativ schnell nach einer Schädigung auch erneuert.

Zusammenfassend darf festgestellt werden, dass es bei einer Gleitbeanspruchung zur Oxidation der Rauheitsspitzen im Kontakt sowie zu einer Oxidation der metallischen Verschleißpartikel kommt und sich mit steigender Temperatur zusätzlich eine vollständige Oxidationsschicht auf den Kontaktflächen ausbildet. Somit steht mehr oxidischer Abrieb für die Kompaktierung von verschleißminimierenden Triboschichten zur Verfügung. Neben der Erhöhung des oxidischen Abriebs bei höheren Temperaturen ist nach [58] eine zunehmende Tendenz zur Kompaktierung und plastischen Deformation der Verschleißpartikel mitverantwortlich für eine Verschleißreduzierung. Aus der Glazeschicht herausgelöste Verschleißpartikel können wieder leichter rekompaktiert werden. Jiang et al. konnten 1995 in [87] mit experimentellen Arbeiten zum Gleitverschleiß von Nimonic $80 \mathrm{~A}$ gegen Nimonic $80 \mathrm{~A}$ bei 20,150 und $250^{\circ} \mathrm{C}$ eine Übergangstemperatur $T_{C}$ von Hoch- zu Niedrigverschleiß bei $250^{\circ} \mathrm{C}$ für eine bestimmte Belastungssituation ausmachen, wobei ein Sintern zu einer Glazeschicht auf der kompaktierten Oxidschicht erkennbar wurde. Diese Beobachtung verdeutlicht den Einfluss der Temperatur auf die Adhäsion zwischen Verschleißpartikeln und der Bildung von tragenden Triboschichten. Eine weitere Arbeit [62] stützt die Beobachtung durch Warmbehandlung von Gleitproben für $90 \mathrm{~min}$ bei $600^{\circ} \mathrm{C}$. Die während des Experiments bei $20^{\circ} \mathrm{C}$ kompaktierten Schichten wurden durch die Warmbehandlung gesintert. 


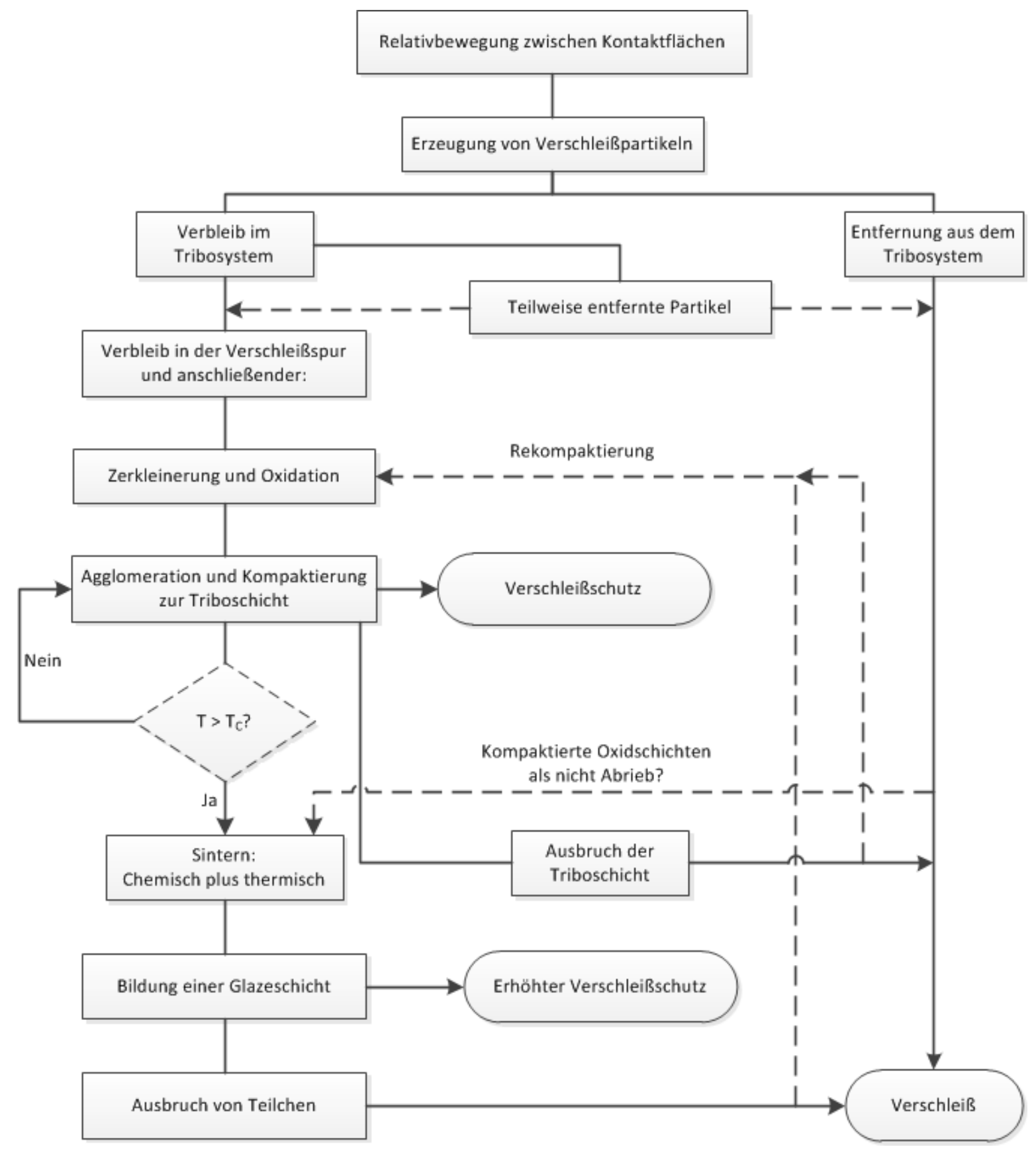

Abbildung 1.8: Schematische Darstellung des Gleitverschleißes mit möglicher
Wiederkompaktierung ausgebrochener Teilchen aus der Glazeschicht nach [88]

Der Effekt von relativ großen Oberflächen kleiner Partikel zur Erhöhung der Oberflächenenergie begünstigt die Adhäsion von Partikeln und somit den Sinterprozess im Reibspalt. Hierdurch wird ein Sintern bei Temperaturen möglich, wo größere Partikel in der konventionellen Pulvermetallurgie nicht gesintert werden können [88]. Adhäsion ist temperaturabhängig, somit darf festgestellt werden, dass eine Erhöhung der Temperatur infolge steigender Umgebungstemperaturen oder induzierter Reibvorgänge die Adhäsion und damit auch das Sintern beschleunigen. Aus experimentellen Ergebnissen führten Jiang et al. [87] ein beschreibendes Modell für den Gleitverschleiß ein. Abbildung 1.8 veranschaulicht das Modell mit möglichen Modifikationen in Bezug auf eine Rekompaktierung von Teilchen aus 
den Glazeschichten sowie einer Entscheidungsbox zur Kontakttemperatur T in Beziehung auf die kritische Übergangstemperatur $T_{C}$ [88].

Nach [89] und [90] beschreibt bislang keine universelle Formel den Gleitverschleiß. Die Adhäsionstheorie von Archard und Hirst [47] und die Delaminationstheorie von Suh [44] berücksichtigen beide nicht den Einfluss von Verschleißpartikeln. Doch wie in diesem Kapitel ausgeführt, bestimmen Entstehung und Verbleib von Verschleißpartikel im Reibspalt das weitere Verschleißverhalten eines Tribosystems.

\subsubsection{Stoßverschleiß}

Auf dem Gebiet des Stoßverschleißes ist die Quellenlage neben dem Buch von Engel [91] und seinen Arbeiten vergleichsweise übersichtlich [92], [93]. Es ist bekannt, dass nach [94] und [95] die vier Faktoren Stoßgeschwindigkeit, Körpermasse, Geometrie der Kontaktbereiche und mechanische Werkstoffeigenschaften den Stoßverschleiß bestimmen. Die Quellenlage umfasst einen weiten Bereich von Werkstoffen, Geometrie und Aufschlagenergien. Rabinowicz schlussfolgerte 1952 in [96] für duktile Werkstoffe mit der Adhäsion den gleichen Verschleißmechanismus unter Schlagbelastung wie unter Gleitreibung. Spätere Arbeiten von De Gee et al. stützen 1964 in [97] die Theorie von Rabinowicz in Bezug auf Gleitverschleiß und Werkstoffübertragung. Hingegen verdeutlichen die Arbeiten von Montgomery aus 1968 in [98], Bayer et al. aus 1972 in [99] sowie Blickensderfer und Tylczak aus 1983 in [100] mit verschiedenen Werkstoffen den gemeinsamen mechanisch-dominierten Verschleißmechanismus Oberflächenzerrüttung. Studman und Field stellten 1977 in [94] radiale sowie umlaufende Risse bei Einzelschlägen und schuppenförmige Ausbrüche bei wiederholter senkrechter und gewinkelter Schlagbelastung an gehärteten Stahlproben fest. Im Bereich der Randschicht konnte eine Kaltverfestigung beobachtet werden. Im Unterschied zu den anderen Arbeiten wurde keine Ermüdung, sondern eine Bildung und Ausbreitung von Rissen als eigenschaftsbestimmender Mechanismus identifiziert. Sorokin und Matyushima untersuchten die Verschleißbeständigkeit von hochkohlenstoffhaltigen Stählen als Funktion der Härte [101], [102]. Man fand heraus, dass die Verschleißbeständigkeit bei kleinen Schlagenergien mit der Härte ansteigt, hingegen fiel die Verschleißbeständigkeit bei größeren Schlagenergien. Khruschov bestimmte später 1974 in [103] für Stahl einen Kohlenstoffgehalt von $0,8 \%$ als Maximum für die höchste Verschleißbeständigkeit unter Schlagbelastung. Wellinger und Breckel untersuchten 1969 in [104] den Einfluss verschiedener Parameter auf den Stoßverschleiß für verschiedene Metalle wie Kupfer, Aluminium und Stahl. Es wurden Oxidation, Schuppenbildung und plastische Deformation festgestellt. Ihre Schlussfolgerungen stützen die Erkenntnisse von Studman und Field mit einer Erweiterung in Bezug auf weichere Werkstoffe. In der tribologisch beanspruchten Randschicht der weicheren Metalle bildet sich eine plastische Deformation aus, in der mit Verschleißbeginn ein plastisches Fließen stattfindet. Wohingegen ein Ausbrechen größerer Partikel bei härteren Werkstoffen eintritt. Bayer et al. beobachteten 1972 in [99] bei Versuchen mit einer Prüffrequenz von $50 \mathrm{~Hz}$ und einem Projektilgewicht von $1 \mathrm{~g}$ aus Stahl auf eine härtere Stahlplatte nach $10^{6}$ Zyklen einen abrupten Verschleiß. 
Die verschiedenen Ergebnisse zusammenzufassen und eine Richtung zu geben, ist aufgrund der Breite an experimentellen Arbeiten nicht eindeutig. Bei hohen Aufschlagskräften führen Rissbildung und Rissausbreitung zu einem Werkstoffverlust insbesondere bei härteren Werkstoffen. Kleinere Aufschlagskräfte bewirken tendenziell eine Ermüdung der Oberfläche, insbesondere bei weicheren Werkstoffe werden plastische Deformationen in der tribologisch beanspruchten Randschicht sichtbar.

\subsubsection{Milder Verschleiß}

Typisch für milden Verschleiß sind Verschleißraten von $\mathrm{nm} / \mathrm{h}$. Derartig kleine Verschleißraten können durch eine vollständige Trennung von Grund- und Gegenkörper mittels Schmierfilm erreicht werden. Zumeist wirken bei mildem Verschleiß andere Verschleißmechanismen als die vier bekannten Hauptmechanismen und sind gegenwärtig Stand der Forschung. Untersuchungen von Scherge et al. 2003 in [105] sowie von Scherge und Pöhlmann 2004 in [106] verdeutlichten, dass sich die chemischen Veränderungen zum Großteil auf den oberflächennnahen Randbereich bis in eine Tiefe von mehreren 100 Nanometern begrenzen. Parallel zu den chemischen Veränderungen laufen kontinuierlich Oberflächenveränderungen im Mikrometerbereich ab. Rigney betrachtet 2000 in [67] plastische Fließvorgänge als Ursache der Mikrostrukturveränderungen, die in überwiegender Zahl als Kornfeinung zu beobachten sind. Damit verbunden sind Veränderungen der mechanischen Eigenschaften in der oberflächennahen Randzone festzustellen (Abbildung 1.9). Verschleißpartikel bei mildem Verschleiß kennzeichnet ein mehrfaches Übergleiten bevor sie den Reibspalt verlassen, wobei sie aus verflüssigtem Material der Randzone stammen. Belin et al. beschreiben 1987 in [107] die Morphologie der Partikel als zumeist flach und abgeplattet, wobei sie zum Großteil eine amorphe Struktur aufweisen.

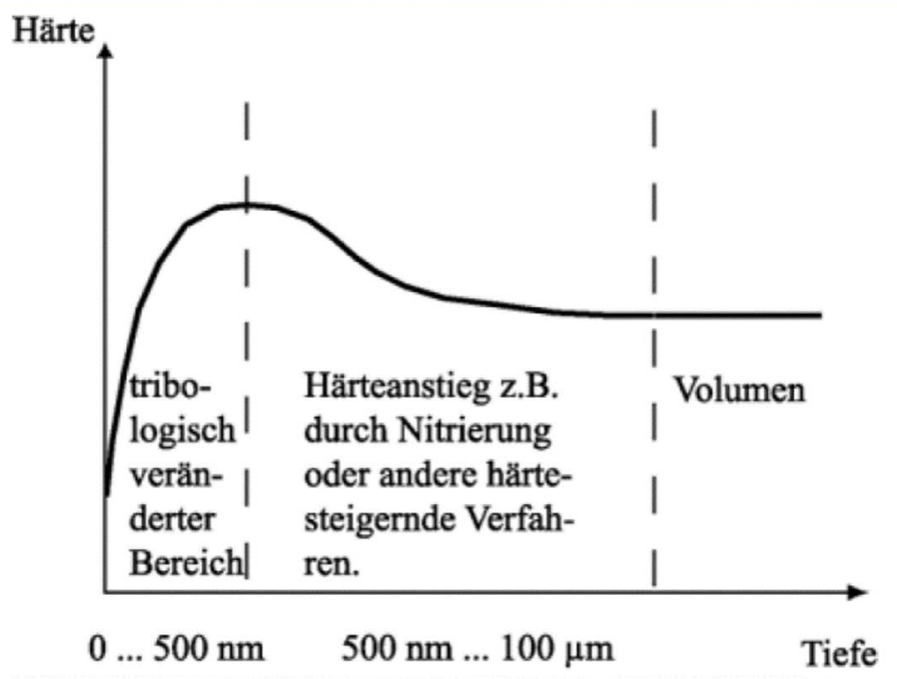

Abbildung 1.9: Qualitativer Härteverlauf über die Tiefe nach [106] 


\subsubsection{Dritter Körper}

Godet führte 1984 in [61] den Begriff des dritten Körpers ein. Nach seinem Verständnis ist der dritte Körper eine trennende Schicht an den Oberflächen von Grund- und Gegenkörper (two „first bodies"), die im Reibkontakt auftritt und eine unterschiedlich chemische Zusammensetzung im Vergleich zu Grund- und Gegenkörper aufweist. Für ein besseres Verständnis über Tribosysteme ist die Kenntnis des dritten Körpers wichtig, da dieser den Verschleiß des Tribosystems entscheidend bestimmt. Der dritte Körper kann aus Verschleißpartikeln und Schmiermittelbestandteilen bestehen. In den Zwischenräumen der Rauheitsspitzen lagern sich Verschleißpartikel ein, wodurch die reale Kontaktfläche vergrößert wird. In der Folge verteilt sich eine wirkende Normalkraft auf eine größere Fläche und somit reduziert sich die Flächenpressung. Geschwindigkeitsdifferenzen zwischen Grund- und Gegenkörper können dadurch vom dritten Körper aufgenommen und der Verbleib von Verschleißpartikel im Reibspalt beeinflusst werden. Damit besitzt der dritte Körper rheologische Eigenschaften. Er ist in der Lage auf kurzen Zeitskalen Kräfte elastisch abzufedern, aber auf längeren Zeitskalen das System wieder zu verlassen. Aufsetzend auf Godet erweiterte Berthier 2005 in [108] die Theorie des dritten Körpers und beschreibt ihn als Material zwischen Grund- und Gegenkörper. Dieses Material wird aus den Reibkörpern infolge einer tribologischen Beanspruchung erzeugt. Er nimmt an, dass bei jeder tribologischen Belastung ein solcher Körper gebildet wird und sich ein schützender Effekt durch Trennung der Reibkörper einstellt. Als Zusammensetzung des dritten Körpers nennt Berthier Oxidschichten, Verschleißpartikel aus den Reibkörpern und Verunreinigungen, wobei Verschleißpartikel die Zwischenräume der Rauheitsspitzen auffüllen und Oxidschichten den Gleitverschleiß reduzieren. Shakhvorostov et al. stimmen 2006 in [109] mit der Theorie von Godet und Berthier im Punkt der Entstehung des dritten Körpers nicht überein. Für die Bildung eines dritten Körpers muss eine bestimmte Energie in das Tribosystem eingetragen werden und ein schneller Einlauf mit kleiner Verschleißrate im stabilen Zustand erfolgen. Die eingebrachte Energie muss ein plastisches Fließen bis zum mechanischem Mischen bewirken, sie darf jedoch nicht zu einem instabilen Zustand mit großen Verschleißraten führen. Der dritte Körper schließt das Material zwischen Grund- und Gegenkörper sowie die mikrostrukturell veränderten Randschichten mit ein. Nach Scherge et al. [105] ist für den Erhalt eines verschleißminimierenden dritten Körpers ein Gleichgewicht zwischen Abtrag und Bildung einer tribologisch induzierten Randschicht maßgeblich. Denn somit kann nach einem guten Einlauf eine kontinuierlich kleine Verschleißrate erreicht werden.

Die Bildung des dritten Körpers ist ein multifaktorieller Prozess und Gegenstand der aktuellen Forschung. Daher ist es gegenwärtig nicht möglich exakt vorherzusagen, unter welchen Randbedingungen ein guter Einlauf erzielt werden kann. 


\subsection{Ventilsystem in 4-Takt-Großgasmotoren}

Der Verbrennungsmotor ist eine periodisch arbeitende Maschine [110]. Eine bestimmte Menge des Arbeitsmediums strömt in den Zylinder ein, verrichtet Arbeit und wird anschließend ausgeschoben. Die Funktion des Ventilsystems ist die Steuerung des Ladungswechsels und das Abdichten des Verbrennungsraums gegenüber den Gaskanälen, wobei die Füllung bei 4-Takt-Großmotoren kein reines Ansaugen ist, sondern mittels Turboaufladung unterstützt wird. Bei 1-stufiger Aufladung werden ca. 5,5 bar und bei 2stufiger ca. 8,0 bar angewendet [111]. Für das Füllen und Entleeren des Zylinders kommen in 4-Takt-Großgasmotoren in überwiegender Zahl je zwei EVSn und AVSn mit den entsprechenden ESRen und ASRen pro Zylinderkopf zum Einsatz. Über die EVSn werden die Einlasskanäle abgedichtet, durch die die Ansaugluft einströmt. Die AVSn steuern die Verbrennungsgase und lassen diese nach dem Arbeitstakt ausströmen. Bevor die Abgase ins Freie gelangen, treiben sie über eine Abgasturbine einen Verdichter an, der die Ansaugluft vor Zylindereintritt vorverdichtet (Turbolader). VS und SR in Großgasmotoren sind Präzisionsteile mit Maßtoleranzen von wenigen Mikrometern und Winkelminuten. In Abbildung 1.10 ist ein typischer Zylinderkopf mit montiertem Ventilsystem dargestellt. Für nähere Informationen zu Arbeitsverfahren, Ladungswechsel oder Aufladung von Großmotoren sei auf die umfangreiche Fachliteratur verwiesen [112], [113], [114], [115] und [116].

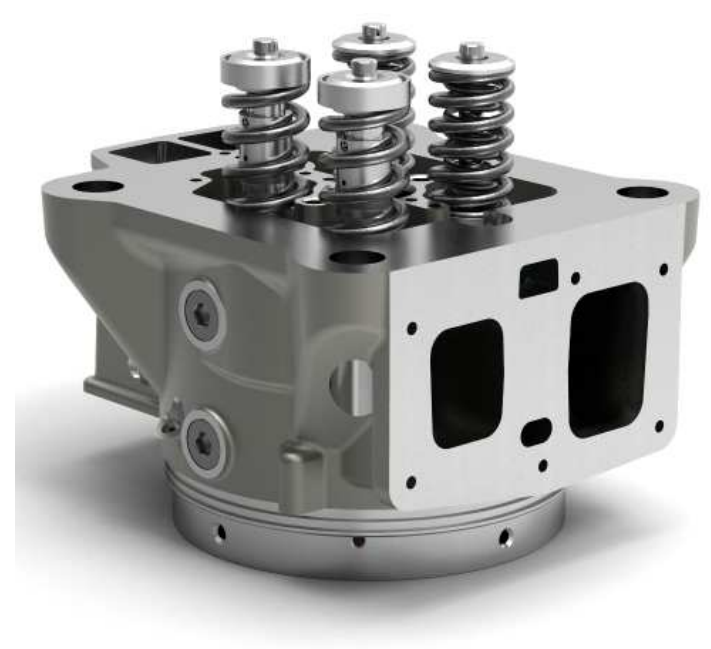

(a)

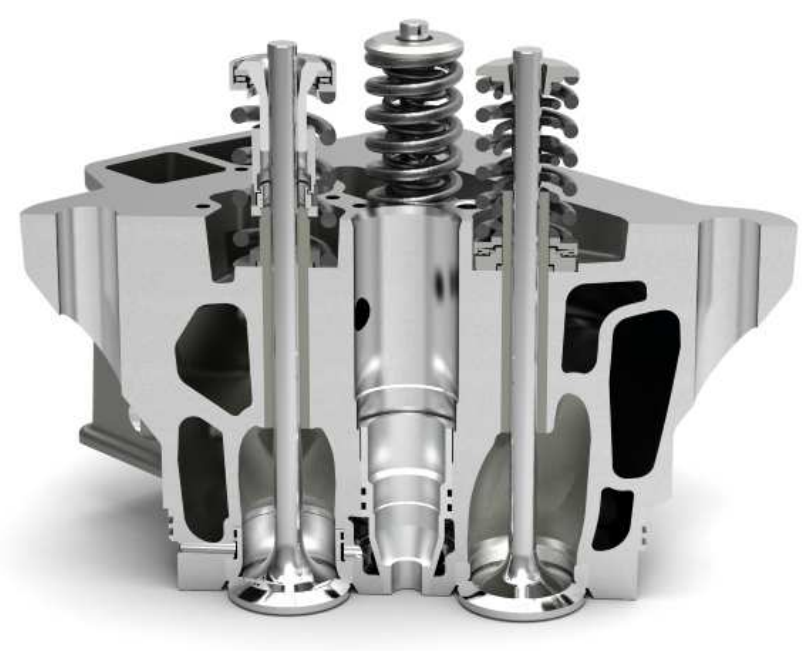

(b)

Abbildung 1.10: (a) Zylinderkopf mit montiertem Ventilsystem, (b) Halbschnitt von (a) mit freundlicher Genehmigung der Märkisches Werk GmbH

In dieser Arbeit wird das Tribosystem VS/SR in Großgasmotoren nach dem Ottoprinzip betrachtet. Diese Technologie wurde durch ein Patent der Gasmotorenfabrik Deutz AG vom Erfinder Nikolaus August Otto am 6. Juni 1876 begründet. Charakteristisch für Ottomotoren ist die Fremdzündung, die in Großgasmotoren mit Direkt- oder Vorkammerzündung erfolgt [115]. In den letzten Jahren gewann der Gasmotor an Bedeutung, da er als umweltfreundlicher, effizienter, stationärer Großmotor im Leistungsbereich von ca. 100 bis 
$100.000 \mathrm{~kW}$ überwiegend zur Stromerzeugung Anwendung fand [117]. Im Vergleich zu Gasturbinen gleicher Leistung übersteigen heutige Großgasmotoren deren Wirkungsgrade und erreichen fast die von Dieselmotoren [115]. Das niedrige Rohemissionsverhalten gegenüber Dieselmotoren stellt ihren größten Vorteil dar. Betrachtet man die gegenwärtig wichtigste Applikation von Großgasmotoren das Kraftwerk, so erzielen Anlagen mit KraftWärme-Kopplung ein in der Größenordnung ähnliches $\mathrm{CO}_{2}$-Äquivalent in $\mathrm{g} / \mathrm{kWh}_{\mathrm{el}}$ wie erneuerbare Energieanlagen (Wind-, Photovoltaik und Wasserkraftwerke) oder Atomkraftwerke [118]. Neben Erdgas kommen biologische Gase wie Klärgas aus Kläranlagen, Deponiegas aus Mülldeponien oder Grubengas aus dem Bergbau sowie Holzgas aus der Holzabfallentsorgung zum Einsatz. Als synthetische Gase werden Kokerei-, Pyrolyse-, Raffinerie- sowie Propan-Butangase und Wasserstoff angewendet. In der Folge variieren im Gegensatz zu Benzin- und Dieselmotoren die Verbrennungsprodukte in weiten Grenzen. Als Folge der in der TA-Luft 2017 festgelegten NO -Grenzwerte für Gasmotoren resultieren die beiden Ansätze für stöchiometrische und magere Gemische als Brennverfahren [115]. Entweder werden $\lambda=1$-Konzepte ( $\mathrm{NO}_{\mathrm{x}}: 250 \mathrm{mg} / \mathrm{m}^{3}$ ) mit aufwendiger Abgasnachbehandlung oder $\lambda>1,6$-Magerkonzepte $\left(\mathrm{NO}_{\mathrm{x}}: 500 \mathrm{mg} / \mathrm{m}_{\mathrm{n}}{ }^{3}\right.$ ) zur Emissionserreichung verwendet. Für $\lambda>$ 1,6-Motoren mit einem Bohrungsdurchmesser $\geq 200 \mathrm{~mm}$, wie sie Gegenstand dieser Arbeit sind, sind für eine sichere Zündung und schadstoffarme Verbrennung besondere Maßnahmen zu ergreifen [115]. Sogenannte Vorkammerzündungskonzepte kommen zur Anwendung, die eine sichere Zündung mit reduzierten Emissionen insbesondere von Ruß- und Aschepartikeln bewirken. Damit einhergehend sind neuartige Betriebsbedingungen zu beobachten. In der Folge reduzieren sich zunehmend für das Tribosystem VS/SR die Verbrennungsrückstände zur Bildung verschleißminimierender Triboschichten. Neben reduzierten Rückständen bedeuten Verbrennungsprozesse mit mageren Gemischen einen Sauerstoffüberschuss, wodurch sich die oxidative Belastung auf VS und SR vermutlich erhöht [60]. Ein möglicher Einfluss auf das Tribosystem VS/SR ist aktuell Gegenstand der Forschung. Zur Verbesserung der Effizienz, welche pro Jahr für Gasmotoren und Dieselmotoren um ca. 0,30 \% bzw. 0,25 \% steigt [119], werden die Zünddrücke, wie eingangs erwähnt, angehoben. Parallel bieten Ventilsteuerzeiten Potenzial zur Erhöhung des Wirkungsgrads durch eine verbesserte Füllung. Miller-ValveTiming im Verbund mit dem Turboladersystem sind dabei entscheidende Technologien [120]. Aus [43] sind Ventilschließgeschwindigkeiten von ca. 0,5 m/s als Stand der Technik bekannt, die jedoch hinsichtlich der Zylinderfüllung begrenzend wirken. Aus Sicht der Thermodynamik werden möglichst große Geschwindigkeiten gefordert [121].

\subsubsection{Ventilspindel und Sitzring}

Als Bauform für Gaswechselventile in 4-Takt-Großgasmotoren haben sich Pilzventile in überwiegender Zahl durchgesetzt [110]. Kennzeichnend für diese Ventile, welche im Großmotorensegment als Ventilspindel bezeichnet werden, ist ein Ventilkopf bestehend aus Ventilteller mit kegeliger Dichtfläche sowie kegelförmigem Übergang (Hohlkehle) zum zylindrischen Ventilschaft. In Abbildung $8.1 \mathrm{im}$ Anhang sind die Bezeichnungen an einer VS dargestellt. Der Gegenkörper Ventilsitzring, kurz Sitzring genannt, ist eine ringförmige 
Komponente mit kegeliger Dichtfläche. Er wird in den Zylinderkopf eingepresst und auslassseitig meist direkt gekühlt. Im Vergleich zu Schieberventilen hat die VS als Sitzventil tribologisch den Vorteil, dass die Kontaktflächen durch Heben geöffnet werden und somit der Gleitkontakt minimiert wird. Abbildung 1.11 veranschaulicht typische Ausführungen von VS und SR für Ein- und Auslass.

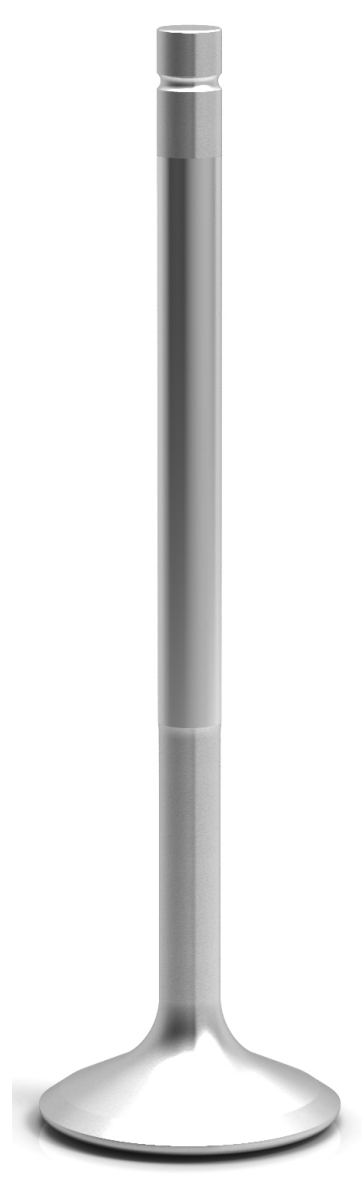

(a)

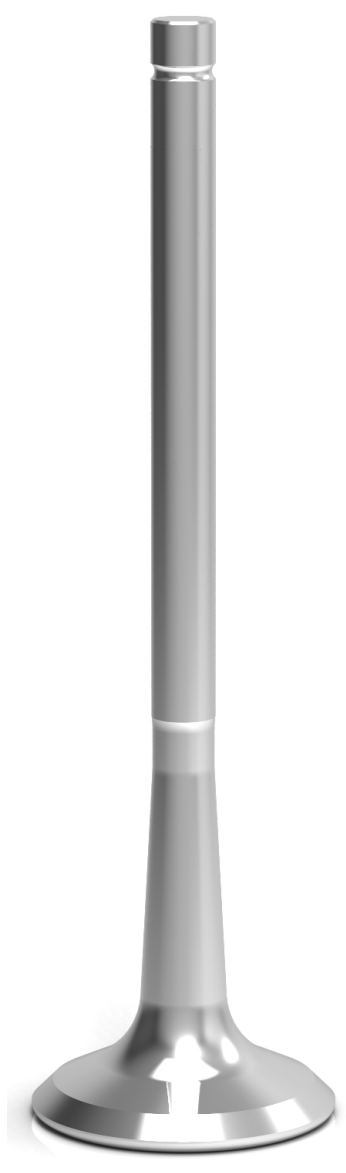

(b)

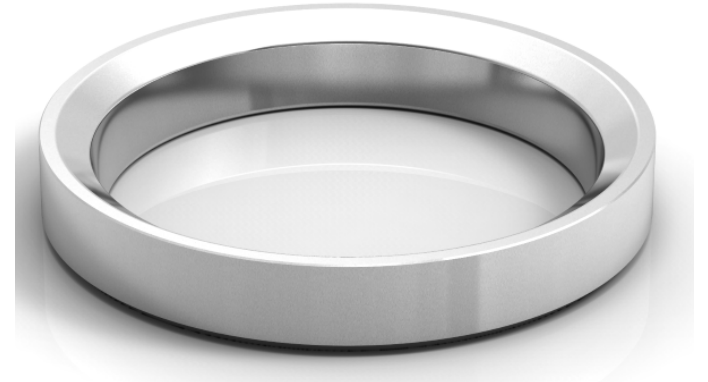

(c)

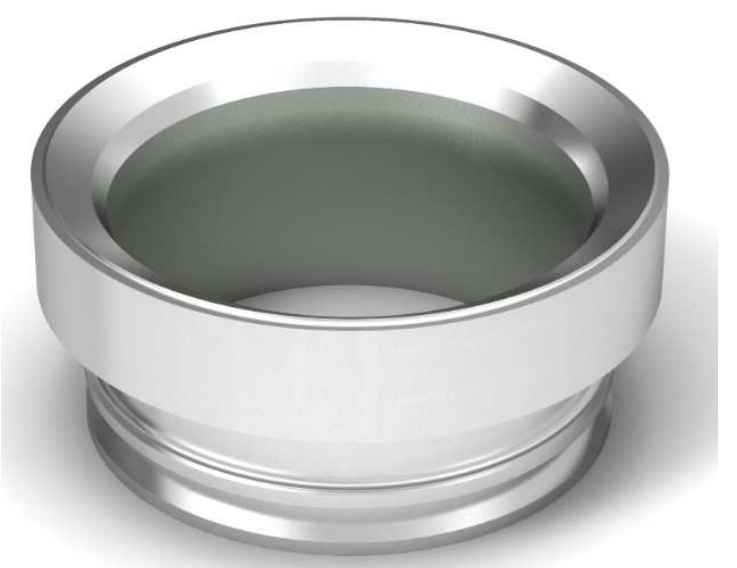

(d)

Abbildung 1.11: Typische Designs für VS und SR; (a) EVS, (b) AVS, (c) ungekühlter ESR und (d) gekühlter ASR mit freundlicher Genehmigung der Märkisches Werk GmbH

Für die Dichtflächen von VS und SR empfiehlt sich ein Differenzwinkel, den man so wählt, dass der Ventilsitz außen, d.h. an der dem Verbrennungsraum zugewandten Seite, durch Linienkontakt zuerst abdichtet. Man spricht in dem Fall von Außentrag. Der Traganteil der Kontaktflächen vergrößert sich unter dem Verbrennungsdruck infolge elastischer Durchbiegung des Ventiltellers. Der Differenzwinkel ist nach Erfahrung des Autors in Abhängigkeit vom Verbrennungsdruck, Arbeitsmedien und Werkstoffen zu wählen. Er sollte min. 5' und max. 40' betragen. Bei Großgasmotoren haben sich in den letzten Jahren Sitzwinkel von $30^{\circ}$ und $20^{\circ}$ etabliert. Anzumerken ist, dass ein Sitzwinkel von $45^{\circ}$ strömungstechnisch die beste Zylinderfüllung bedeutet. Neben dem Sitzwinkel ist aus konstruktiver Sicht der Aspekt des Ventilüberhangs von Bedeutung. Primär bestimmt durch 
die Wahl der Werkstoffe und mechanischen Kontaktbedingungen kann der Überhang Null bis mehrere Zehntelmillimeter im Einzelfall bis wenige Millimeter betragen. Studien zu diesem Thema finden sich in der wissenschaftlichen Literatur nicht. Abbildung 1.12 veranschaulicht das Ventilsystem, wie es in dieser Arbeit verwendet wird, bestehend aus VS und SR mit den Details Differenzwinkel und Überhang.

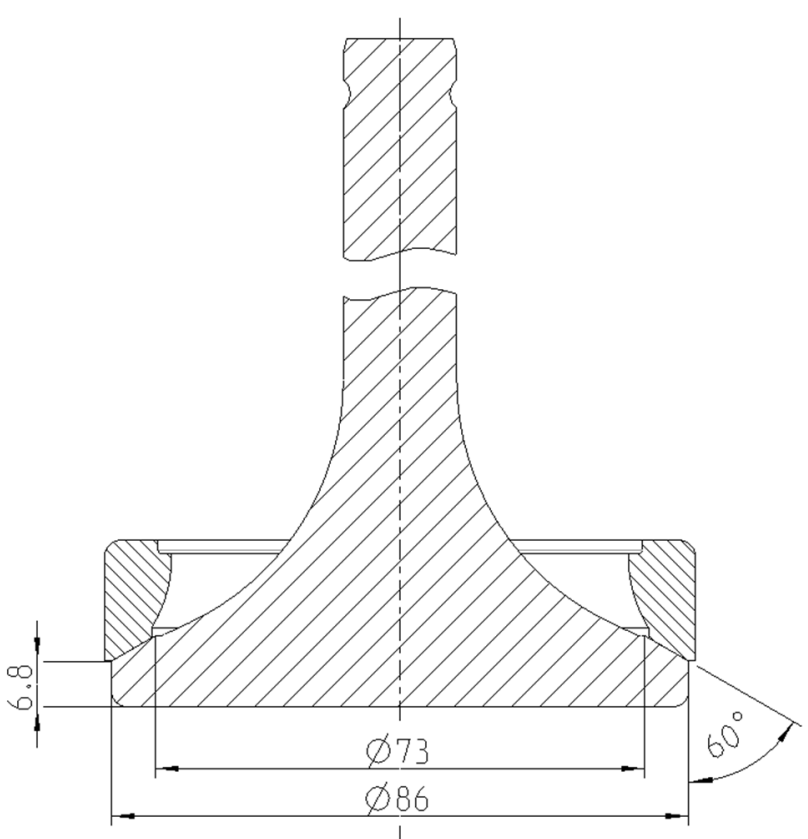

(a)

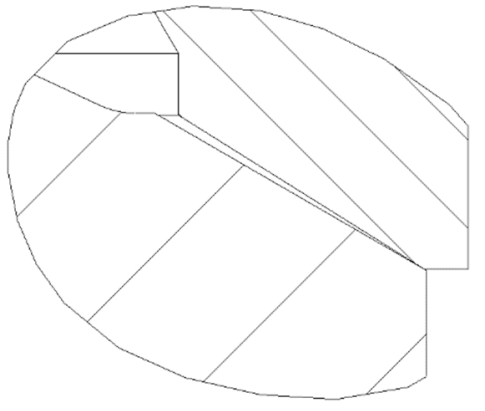

(b)

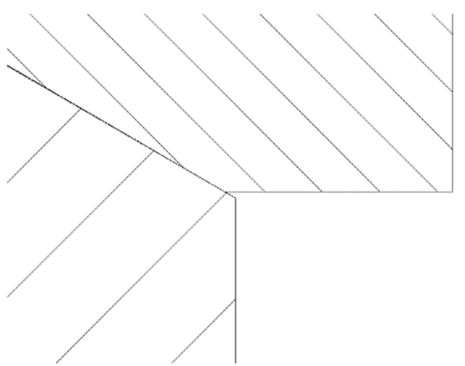

(c)

\section{Abbildung 1.12: (a) Ventilsystem bestehend aus VS und SR, (b) Detail aus (a) mit Differenzwinkel, (c) Detail aus (a) mit Ventilüberstand}

In überwiegender Zahl bewegt eine untenliegende Nockenwelle, welche über schrägverzahnte Zahnräder von der Kurbelwelle angetrieben wird, den Ventiltrieb in Großmotoren. EVS und AVS werden indirekt über Stößel, Stößelstange, Ventilbrücke und Ventilkipphebel geöffnet und geschlossen [116], wobei der Kipphebel auf das Ventilschaftende drückt. Die rotatorische Bewegung der Nockenwelle wird über den Ventiltrieb in eine translatorische Bewegung der VS umgewandelt. Die Nockenform definiert den Verlauf der Ventilbewegung, wobei die VS hinsichtlich der Zylinderfüllung schnellstmöglich Öffnen und Schließen sollte. Abbildung 1.13 veranschaulicht normierte Ventilerhebungskurven für EVS und AVS sowie Zylinderdruck über $720^{\circ} \mathrm{KW}$. Dabei sind die Erhebungskurven so zu wählen, dass keine unzulässigen Schwingungen angeregt werden. Ohne hier auf eine nähere Betrachtung der Schwingungsberechnung einzugehen, sei angemerkt, dass der Ventiltrieb mit seiner Masse und Federn ein schwingungsfähiges System darstellt. Die Eigenfrequenz sollte so hoch wie möglich gewählt werden. Der Nocke initiiert die Erregungsfrequenz. Aufgrund des Ventilspiels mit Entkopplung von VS und Kipphebel kann sich kein stationärer Schwingungszustand einstellen, da kein Kraftschluss mehr besteht. Die Schwingungen enden. Mit zunehmenden Ventilverschleiß reduziert sich das Ventilspiel und die Gefahr eines Kraftschlusses steigt. Ist kein Ventilspiel mehr gegeben, kann der Ventiltrieb 
in Resonanz kommen und ein katastrophaler Motorschaden die Folge sein. Ein Ventilzyklus besteht aus den drei Phasen Ventilöffnen, Ventilschließen und Verbrennungsdruck (Abbildung 1.13). Während des Öffnens vergrößert sich der Nockenradius, die Brücke komprimiert die Ventilfedern und drückt die VS nach unten. VS und SR werden entkoppelt. Nach Erreichen des maximalen Ventilhubs verkleinert sich der Nockenradius. In der Folge wird das Ventilfederpaket dekomprimiert und zieht die VS in Richtung SR. Mit Erreichen des kleinsten Nockenradius setzt die VS auf den SR auf. VS und SR werden gekoppelt. Die lineare Bewegung des Ventilschafts in der Ventilführung wird durch eine Verlustschmierung vom Schaftende geschmiert. Diese Verlustschmierung ist eine wesentliche Quelle zur Bildung einer Triboschicht auf den Kontaktflächen von VS und SR.

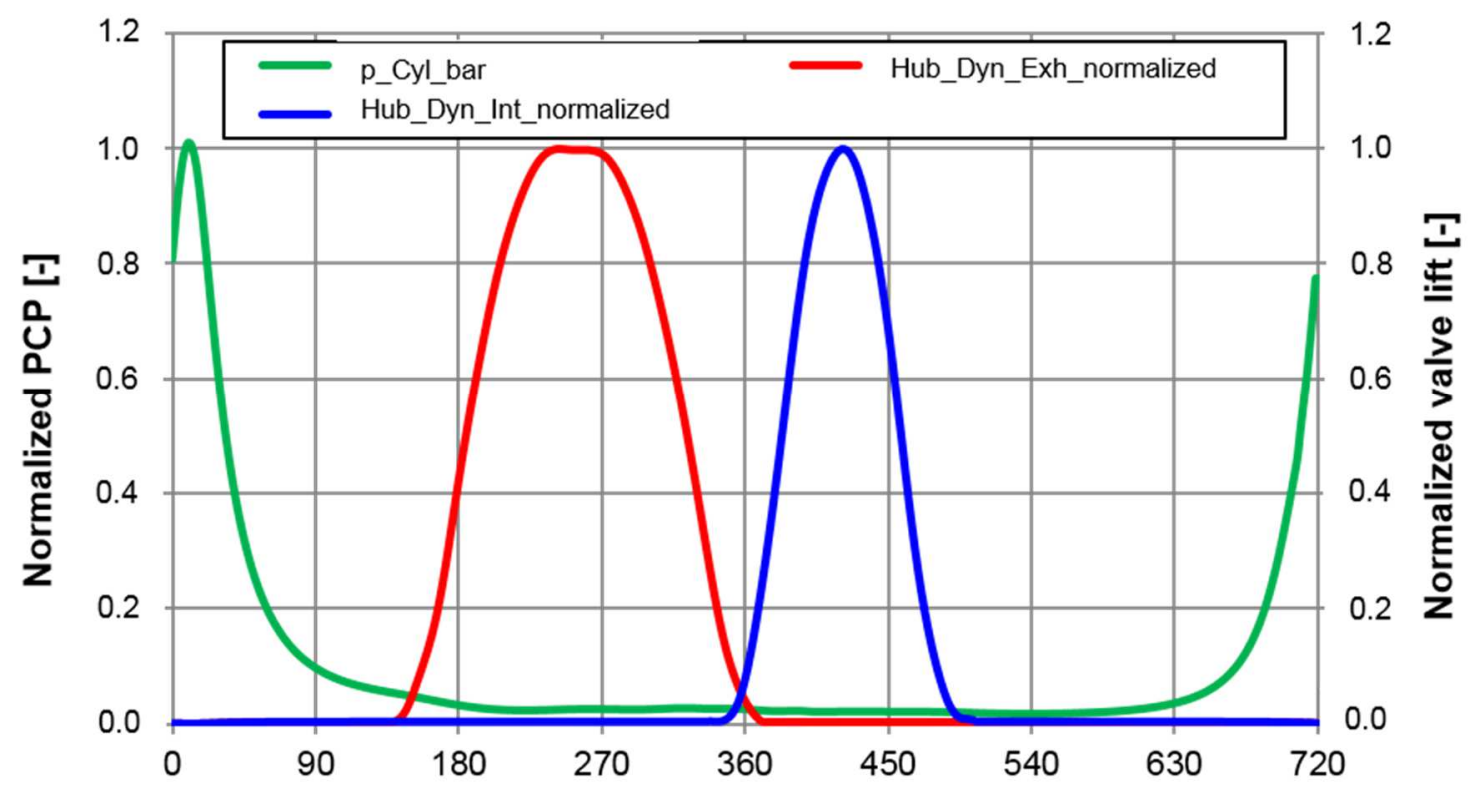

Abbildung 1.13: Normierte Darstellung der Ventilerhebungskurven für EVS (rot) und AVS (blau) und Zylinderdruck (grün) über $720^{\circ} \mathrm{KW}$

Die Anforderungen an die Lebensdauer von VSn betragen in den Bereichen Automotive ca. $250.000 \mathrm{~km}$ (entspricht ca. 4.200 Betriebsstunden bei $60 \mathrm{~km} / \mathrm{h}$ ), Heavy-Duty ca. $1.600 .000 \mathrm{~km}$ (entspricht ca. 26.700 Betriebsstunden bei $60 \mathrm{~km} / \mathrm{h}$ ) [122] und Großmotor ca. 40.000 Betriebsstunden. Generell erfolgt die Dimensionierung von VS und SR abhängig von Zylinderdurchmesser, Verbrennungs- und Turboladerdruck, freier oberer und unterer Ventillänge, Kraftstoff sowie Zylinderkopfstruktur. Der Tellerdurchmesser sollte für eine gute Füllung möglichst groß dimensioniert werden. Begrenzend wirken Zylinderkopf- und Brennraumdesign. Parallel angeordnete Ventile haben kleinere Tellerdurchmesser als $V$ förmig angeordnete, hierfür wählt man allgemein:

Fall 1 Ventile gleich groß:

$$
d_{T} \approx 0,4 \times D
$$

Fall 2 Ventile unterschiedlich groß:

$$
d_{T_{-} I N} \approx 0,45 \times D
$$




$$
d_{T_{-} E X} \approx 0,35 \times D
$$

mit Tellerdurchmesser $\mathrm{d}_{\mathrm{T}}$, Zylinderdurchmesser D, Einlass IN und Auslass EX.

Die Abmessungen des Schaftdurchmessers werden gewählt mit:

Motoren mit großer Drehzahl: $\quad d_{S_{-} \text {groß }} \approx(0,25$ bis 0,35$) \times d_{T}$

Motoren mit kleiner Drehzahl: $\quad d_{S_{-} \text {klein }} \approx(0,15$ bis 0,25$) \times d_{T}$

Abhängig vom Tellerdurchmesser $d_{T}$ ergibt sich für den maximalen Ventilhub $h_{\max }$ :

$$
\pi \times d_{i} \times h_{\max }=\pi \times d_{i}^{2} / 4
$$

mit $\quad d_{i} \approx d_{T}$

$$
h_{\max } \approx d / 4
$$

Aus der Erfahrung liegt der maximale Ventilhub $h_{\max } z$ wischen $d_{T} / 6$ und $d_{T} / 4$.

Nach der Dimensionierung der Ventilabmessungen wird der Tellerdurchmesser und der maximale Ventilhub $h_{\max }$ mit der mittleren Strömungsgeschwindigkeit $w_{m}$ am Ventilsitz überprüft:

$$
w_{m} \times \cos \alpha \times \pi \times d \times h_{\max }=c_{m} \times \pi \times D^{2} / 4
$$

mit Ventilsitzwinkel $\alpha$, Geschwindigkeitskomponente $w_{m} \cos (\alpha)$ senkrecht zum Querschnitt $\pi d_{T} h_{\text {max }}$, mittleren Kolbengeschwindigkeit $c_{m}$ und Zylinderdurchmesser D.

$$
\begin{gathered}
w_{m}=c_{m} \times \frac{D^{2}}{4 \times d_{T} \times h_{\max } \times \cos \alpha} \\
A_{v}=\pi \times d_{T} \times h \times \cos \alpha
\end{gathered}
$$

Erfahrungswerte für die mittlere Strömungsgeschwindigkeit $w_{m}$ liegen für EVS und AVS bei ca. 60 bis $90 \mathrm{~m} / \mathrm{s}$ bzw. ca. 80 bis $120 \mathrm{~m} / \mathrm{s}$. Verkleinert man den Sitzwinkel von 45 auf $30^{\circ}$ ergibt 
sich nach Gleichung (1.22) ein Widerspruch, da rechnerisch eine Verringerung der Strömungsgeschwindigkeit $w_{m}$ resultiert und sich somit eine Verbesserung der Strömungsverhältnisse einstellen würde. In der Praxis ist nahezu keine Vergrößerung der Füllung zu verzeichnen [110]. Auslassseitig lässt sich eine schnellere Entleerung feststellen. Der Widerspruch erklärt sich aus der vereinfachten Annahme für den offenen Ventilquerschnitt $A_{v}$, da die Gleichung (1.23) den wahren offenen Querschnitt am Ventil nur ungenau wiedergibt. Für die exakte Berechnung des freien Öffnungsquerschnitts sei auf die einschlägige Literatur verwiesen [123]. Für längere Lebensdauern von VSn werden in Großmotoren Ventildrehvorrichtungen eingesetzt, um lokale Erwärmungen am Ventilteller und Ablagerungen am Ventilschaft zu vermeiden.

\subsubsection{Werkstoffe}

Ventilstähle und -legierungen für Verbrennungskraftmaschinen sind in der EN 10090:1998 standardisiert. Neben martensitischen Stählen kommen nichtmagnetische austenitische Werkstoffe zur Anwendung. Zu den letzteren gehören austenitische Edelstähle und NickelSuperlegierungen. Verbrennungsprozess und Ladungswechsel bewirken höchste thermomechanische Belastungen sowie einen Verschleiß- und Korrosionsangriff durch die Verbrennungsprodukte. Aus der unterschiedlichen Funktion von EVS und AVS ergeben sich unterschiedliche Temperatur- und Korrosionsbelastungen, die Auswirkungen auf Dimensionierung und Werkstoffauswahl haben. Während der Kolben die frische Ladung ansaugt, wird die EVS gekühlt, thermomechanisch resultiert ein Thermoschock. In den folgenden beiden Takten Verdichten und Verbrennen wird die EVS erwärmt. Schiebt der Kolben nach beendetem Arbeitstakt das Abgas raus, wird die AVS insbesondere die Hohlkehle erwärmt. Im Vergleich zu Dieselmotoren liegen die Abgastemperaturen von Gasmotoren um ca. $120 \mathrm{~K}$ höher [124]. Am Ventilteller können unter Volllast bei der AVS bis zu $850^{\circ} \mathrm{C}$ [125] und bei der EVS bis zu $500{ }^{\circ} \mathrm{C}$ [110] erreicht werden. Beddoes berichtet in [126] von 500 bis $600{ }^{\circ} \mathrm{C}$ für VS und 300 bis $350^{\circ} \mathrm{C}$ für SR an der Oberfläche. Sicher ist, dass die VS im Betrieb mehrere Hundert Grad wärmer als der SR wird. Dies erklärt sich aus der größeren direkten Bauteilfläche zum Verbrennungsraum. Zusätzlich ist der SR näher am Kühlmedium angeordnet. Daher empfehlen sich für die EVS und AVS martensitische Stähle bzw. austenitische Edelstähle mit Cr-Gehalten bis $20 \mathrm{Gew} .-\%$ [126]. Zur Verbesserung der Verschleißbeständigkeit haben sich im Bereich des Ventilsitzes die Technologien induktives Härten, Kalt- und Warmverfestigen, Nitrieren [127] und Panzern [128], [129] oder Verfahrenskombinationen [130] etabliert. Aus Erfahrung des Autors sind nitriert oder gepanzert ausgeführte Sitzbereiche in Großgasmotoren Stand der Technik. Im Großmotorenbereich werden aufgrund der Möglichkeit zur Überholbarkeit von Zylinderköpfen in überwiegender Zahl SRe verwendet [131]. I.d.R. kommen bis zu 3 Übermaßstufen zum Einsatz. Ferner ist die höhere Verschleißbeständigkeit von SRen gegenüber dem Zylinderkopfmaterial ein weiteres wichtiges Argument. Zum Großteil werden Guss- und Knetlegierungen aus Eisenbasis eingesetzt, aber auch Kobalt- und Nickellegierungen werden verwendet [131]. Pulvermetallurgisch hergestellte SRe, wie sie in den Bereichen 
Automotive oder Heavy-Duty etabliert sind, finden im Großmotorenbereich aufgrund der Stückkosten keine Anwendung. Mit zunehmender Bohrungsgröße gewinnen gepanzerte SRe gegenüber kostenintensiven Gusslegierungen an Bedeutung. Für diese Typen von ESR und ASR werden häufig martensitische Stähle wie 1.4718 oder Vergütungsstähle wie 1.7227 als Grundwerkstoff eingesetzt. Für die Sitzpanzerung haben sich Hartlegierungen durchgesetzt. Alle Kompositwerkstoffe verfolgen den Ansatz einer Partikelverstärkung in einer zähen Metallmatrix [132]. In hochkorrosiver Umgebung werden tendenziell Hartlegierungen aus Nickelbasis im Sitzbereich gepanzert. In überwiegender Zahl sind Stellite ${ }^{\mathrm{TM}}$-Legierungen zu finden. Typisch für die eingesetzten Stellite ${ }^{\mathrm{TM}}$-Legierungen wie Stellite ${ }^{\mathrm{TM}} 6$ oder 12 mit untereutektischer Erstarrung sind die dendritisch ausgebildeten Chrom- und Wolframkarbide eingebettet in eine Co-Cr-Matrix, wobei Chrom als Hartstoffbildner und Korrosionsschutz in der Matrix dient [131]. Die Härte generiert sich primär aus dem Metalloid Kohlenstoff. Parallel haben in den letzten Jahren die intermetallischen Legierungen der Tribaloy ${ }^{\mathrm{TM}}$-Familie an Bedeutung gewonnen. Vergleichende Untersuchungen von Stellite ${ }^{\mathrm{TM}}-12$ und Tribaloy ${ }^{\mathrm{TM}}-\mathrm{T} 400$ verdeutlichen kleineren Verschleiß für Tribaloy ${ }^{\mathrm{TM}}-\mathrm{T} 400$ [60]. Charakteristisch für Tribaloy ${ }^{\mathrm{TM}}$ T400 ist die übereutektische Erstarrung mit eingebetteten intermetallischen CoMoSiHartphasen (Laves-Phasen) in eine Co-Cr-Matrix und lamellarem Eutektikum. Im Unterschied zur stengeligen Hartphasenmorphologie von Stellite ${ }^{\mathrm{TM}}-12$ sind die primären Laves-Phasen rundlich und deutlich größer.

Aus Gründen der Vollständigkeit sollen an der Stelle Gaswechselventile aus Keramik, Titan und Titanaluminiden erwähnt werden. Alle drei offerieren Massevorteile im Vergleich zu VSn aus Eisen- und Nickelbasis. Vollkeramische Ventile bspw. aus Siliziumnitrid bieten zusätzlich Temperaturvorteile [133]. Es darf angenommen werden, dass die drei Varianten aus Kostengründen bislang nicht den Weg in das Großmotorensegment geschafft haben. Im Fall der vollkeramischen Ventile steht zusätzlich die werkstoffbedingte Sprödigkeit dem robusten Einsatz in Großmotoren entgegen.

\subsubsection{Ventilverschleiß}

Der Ventilverschleiß begleitet den Verbrennungsmotor vermutlich seit den ersten Tagen seiner Erfindung. Bereits Aitchinson berichtete 1919 in [134] vom Ventilversagen aufgrund hohen Verschleißes. Obwohl in den vergangenen rund 100 Jahren eine Vielzahl von Untersuchungen zum Ventilverschleiß durchgeführt wurden, konnte die Lösung des Problems nicht gefunden werden. Ventilverschleiß führt zu einem vorzeitigem Funktionsausfall des Tribosystems VS/SR infolge Werkstoffverlust [43]. Es kommt zu einem Rückgang der VS in den SR (Abbildung 1.14). Daher soll unter dem Begriff Ventilverschleiß in dieser Arbeit der Verschleiß der im Kontakt stehenden Oberflächen von VS und SR verstanden werden. Die Zuordnung des Werkstoffverlusts ist nicht eindeutig; manchmal ist der Verlust an der VS, manchmal am SR größer. Sitzt die VS nicht korrekt, kommt es zu einem Verlust an Zylinderdruck und die entweichenden heißen Verbrennungsgase verursachen nach kurzer Zeit Durchbläser, die zu Ventilversagen führen. Die Ursachen des Ventilverschleißes sind bislang nicht vollständig verstanden. Die Verschiedenheit von Kraftstoff, Applikation, Arbeitsprinzip, 
Werkstoff oder Verbrennungsprozess lassen den Ventilverschleiß zu einem multifaktoriellen System werden.

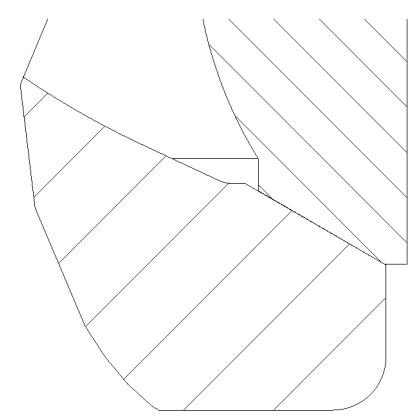

(a)

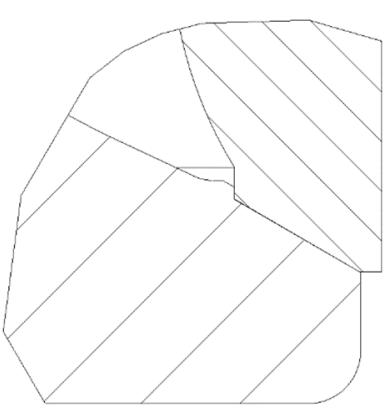

(b)

\section{Abbildung 1.14: Ventilsitz, (a) neu, (b) mit Verschleiß (Ventilrückgang)}

Im Folgenden werden die verschleißbestimmenden Vorgänge des Tribosystems VS/SR näher betrachtet. Es sei angemerkt, dass die wissenschaftlichen Arbeiten zum Ventilverschleiß in überwiegender Zahl die beiden Applikationen Automotive und Heavy-Duty betreffen. Hingegen betrachtet diese Arbeit den Ventilverschleiß in Großgasmotoren. Nichtsdestotrotz geben die Arbeiten Hinweise von den grundlegenden Wirkmechanismen und verdeutlichen die tribologisch komplexe Beanspruchung. In der Literatur werden dem Ventilverschleiß zwei dominierende Verschleißarten zugeordnet [43]. Die Verschleißart Stoßverschleiß resultiert aus dem Auftreffen der VS auf den SR während des Ventilschließens. Daneben wirkt als zweite Verschleißart der Gleitverschleiß infolge elastischer Durchbiegung des Ventiltellers. Zum Thema Ventilöffnen finden sich keine Untersuchungen, daher wird das Entkoppeln von VS und SR als unkritisch angenommen. Der Stoßverschleiß infolge Ventilschließen wird signifikant durch die Faktoren Ventiltriebsdynamik insbesondere Schließgeschwindigkeit sowie Ventilmasse und Koaxialität von VS- sowie SR-Achse bestimmt. Bereits Heron und Beall erkennen 1937 in [135] den SR-Verzug in luftgekühlten Flugzeugmotoren als Ursache von Ventilverschleiß, woraus höhere Schließgeschwindigkeiten und lokale Spannungsspitzen resultieren können. Die Autoren sahen in flexiblen SRen einen Lösungsansatz. Newton und Tauschek schlussfolgern 1953 in [136], dass das Einschlagen der VS in den SR eine wichtige Ursache von Ventilverschleiß aufgrund fehlender Koaxialität von VS- und SR-Achse ist, da sich die Flächenpressung invers zur Kontaktfläche verhält. Abbildung 1.16 (b) veranschaulicht eine fehlende Koaxialität von VS- und SR-Achse. Fricke und Allen stützen 1993 in [137] die vorangegangenen Arbeiten und betrachten die Phase Ventilschließen mit wiederholtem Aufschlagen der VS auf den SR als wichtigsten Einflussfaktor. Weitere Studien zum Einfluss der Koaxialität von VS- und SR-Achse unterstreichen die Signifikanz des Schließvorgangs [52], [138], [139]. Nach [18] definiert sich Stoßverschleiß als Verschleiß von zwei zusammenprallenden Oberflächen mit senkrecht zu ihren Kontaktflächen gerichteten großen Geschwindigkeiten. Der Zusammenstoß von VS und SR im Moment des Erstkontakts während des Ventilschließens führt zu einer impulsförmigen und energetischen Wechselwirkung im Tribosystem VS/SR. In Abbildung 1.15 ist die Aufschlagskraft über die Schließgeschwindigkeit für eine $1276 \mathrm{~g}$ schwere VS aufgetragen, wie sie in dieser Arbeit verwendet wurde. Die 
ermittelten Werte geben einen Hinweis von den auftretenden Kräften mit steigender Ventilschließgeschwindigkeit.

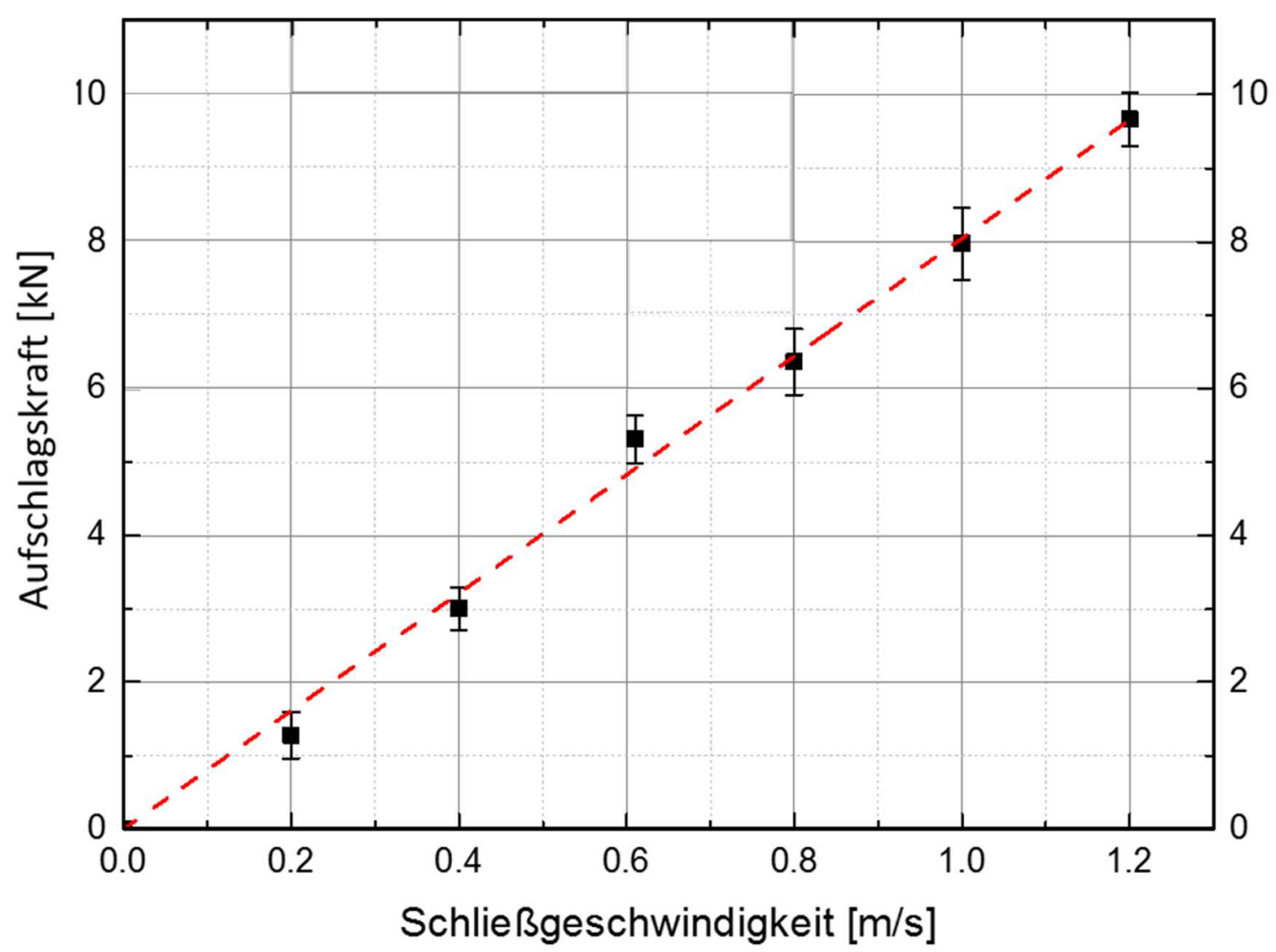

\section{Abbildung 1.15: Aufschlagskraft als Funktion der Ventilschließgeschwindigkeit}

Der Impuls $\mathrm{p}$ ist definiert als:

$$
p=m \times v
$$

mit der Masse $m$ und der Geschwindigkeit v eines Körpers. Für die Geschwindigkeitsänderung eines Körpers muss sein Impuls geändert werden. Der Quotient aus Impuls p und Stoßzeit dt ergibt den übertragenen Impuls, der im Fall des Tribosystems VS/SR der Aufschlagskraft $F_{\text {Schlag }}$ entspricht:

$$
F_{\text {Schlag }}=\frac{d p}{d t}=\frac{m \times v}{t_{2}-t_{1}}
$$

Für eine Ventilmasse von $1276 \mathrm{~g}$ bei einer Schließgeschwindigkeit von $0,6 \mathrm{~m} / \mathrm{s}$ und einer gemessenen Aufschlagskraft von $5400 \mathrm{~N}$ ergibt sich eine Stoßzeit $\mathrm{t}_{\text {stoß }}$ mit $\mathrm{t}_{1}=0$ von 0,14 ms. 


$$
\begin{gathered}
t=\frac{m \times v}{F_{\text {Schlag }}}=\frac{1,276 \mathrm{~kg} \times 0,6 \mathrm{~m}}{s 5400 \mathrm{~N}}=14 \times 10^{-5} \mathrm{~s} \\
e=\frac{v_{2}^{*}-v_{1}^{*}}{v_{1}-v_{2}}
\end{gathered}
$$

Die Stoßzahl e kennzeichnet den Anteil der kinetischen Energie. Für den ideal elastischen Stoß gilt $\mathrm{e}=1$, hingegen wird bei $\mathrm{e}=0$ die gesamte kinetische Energie dissipiert. Das bedeutet für das Tribosystem VS/SR, dass die gesamte Aufschlagskraft $F_{\text {Schlag }}$ als Werkstoffbelastung dissipiert wird. In Verbindung mit dem Aufsetzen der VS ist das Phänomen des Valve Bouncings bekannt [140]. Abbildung 1.16 (a) veranschaulicht den Ventilhub über Grad Kurbelwinkel mit Valve Bouncing im Bereich $180^{\circ} \mathrm{KW}$. Der Begriff Valve Bouncing beschreibt das Zurückprallen der VS vom SR nach dem ersten Auftreffen, wobei ein mehrfaches Entkoppeln, meist zwei- bis dreimal, erfolgen kann. Ursachen können eine Durchbiegung oder Torsion der Nockenwelle, zu hohe Schließgeschwindigkeiten, unzureichende Steifigkeiten der Ventilfedern oder Elastizitäten im Ventiltrieb sein. Die dissipierte Stoßenergie bewirkt im Kontaktbereich neben lokalen Formänderungen wie plastischen Deformationen auch Stoffveränderungen als Folge der mechanischen Aktivierung, die zu Temperaturerhöhungen führen und somit tribochemische Reaktionen fördern können [14].

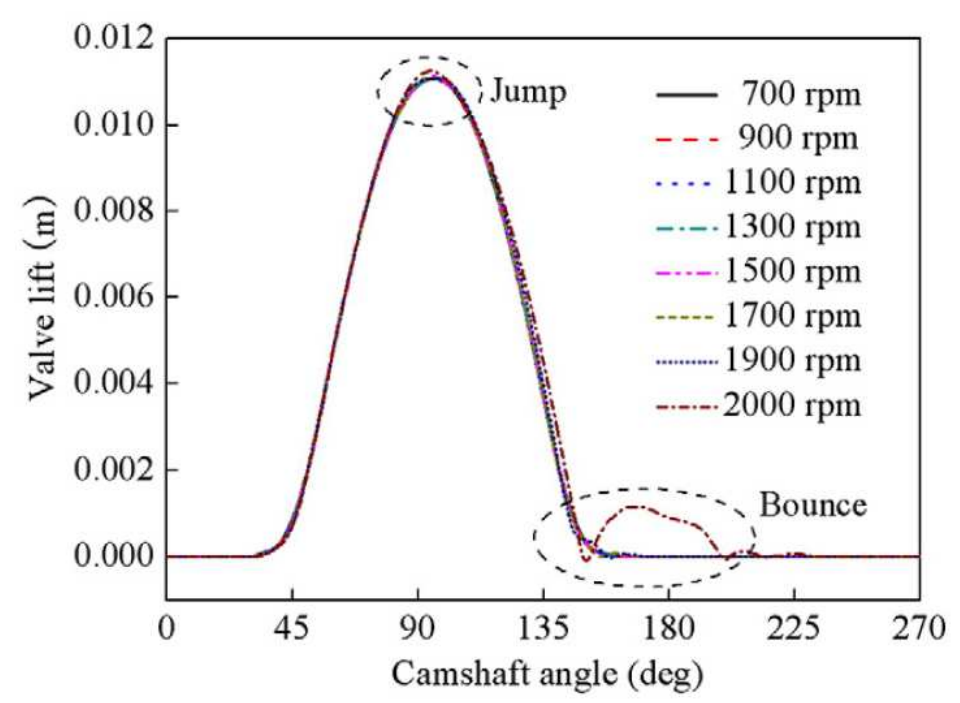

(a)

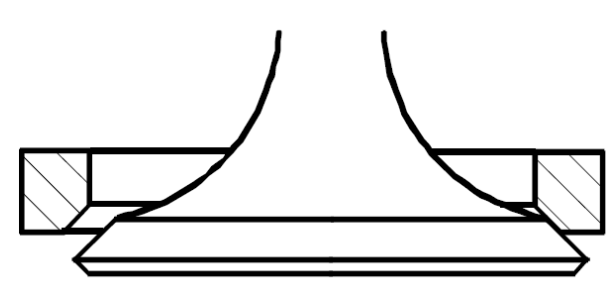

(b)

Abbildung 1.16: (a) Valve Bouncing nach [140], (b) außermittiger Sitz aufgrund Nichtkoaxialität von VS- und SR-Achse nach [43]

Ootani et al. untersuchten 1995 in [141] den Einfluss von Gleitgeschwindigkeit und Temperatur auf den Stoßverschleiß an Luft detaillierter. Modellversuche mit Ring- und Scheibenproben verdeutlichten keinen eindeutigen Trend. Bei Raumtemperatur wiesen die Ringproben und bei $200{ }^{\circ} \mathrm{C}$ die Scheibenproben die größten Verschleißvolumina auf. Bei $400{ }^{\circ} \mathrm{C}$ wurden bei beiden Proben aufgrund der hohen Oberflächenoxidation, so vermuten die Autoren, die kleinsten Volumina gemessen. Lewis et al. berichten 1998 in [52] von einem 
rapiden Anstieg des Verschleißes von VS und SR nach Erweiterung der experimentellen Versuche zum Verbrennungsdruck um das Ventilschließen. Spätere Arbeiten von Chun et al. 2007 in [142] und Slatter et al. 2009 in [143] stützen den signifikanten Einfluss der Schließgeschwindigkeit.

Der Gleitverschleiß infolge Verbrennungsdruck wird signifikant durch die Faktoren Ventiltellergeometrie, Verbrennungsdruck und Koaxialität von VS- und SR-Achse bestimmt [138]. Zinner nahm 1963 in [144] zunächst das Ventilschließen als wichtigsten Faktor für den Ventilverschleiß an. In der Schlussfolgerung seiner Untersuchungen unter verschiedenen Belastungsbedingungen insbesondere zum mittleren Arbeitsdruck korrigierte er seine These und sah einen größeren Einfluss der Gleitreibung infolge Verbrennungsdruck. Pope stützte 1967 in [145] die These von Zinner. Seine Ergebnisse von 20fach höheren gemessenen Spannungen im Ventilteller unter Verbrennungsdruck im Vergleich zum Ventilschließen erweiterten die Problemursache durch die Erkenntnis, dass der Ventilverschleiß infolge einer Relativbewegung zwischen den Kontaktflächen von VS und SR unter Einfluss des Verbrennungsdrucks bewirkt wird. Mathis et al. berichten 1990 in [146] infolge Verbrennungsdrucks von einem Anstieg der Flächenpressung und einem Mikrogleiten von wenigen Mikrometern zwischen den Kontaktflächen von VS und SR. Als Ursache wird die elastische Durchbiegung des Ventiltellers unter Verbrennungsdruck gesehen. Wang et al. untersuchten 1996 in [147] den Effekt von Zyklenanzahl, Verbrennungsdruck und Temperatur. Man fand heraus, dass der Verschleiß mit steigendem Verbrennungsdruck zunimmt. Hingegen wurde ein reduzierter Verschleiß mit steigenden Temperaturen beobachtet. Die Autoren führen dies auf die Bildung von metallischen Oxidfilmen auf den Kontaktflächen zurück. Forsberg et al. stützten 2014 in [148] die These von Mathis et al. und ermittelten aus experimentellen Komponententests an polierten Proben einen proportionalen Zusammenhang zwischen Gleitweg und Verbrennungsdruck. Es wurden Gleitwege von $6 \mu \mathrm{m}$ bei 60 bar und $13 \mu \mathrm{m}$ bei 200 bar für denselben Ventiltyp ermittelt.

Wie erwähnt, werden in Großmotoren Ventildrehvorrichtungen für eine homogene Temperaturverteilung im Ventilteller verwendet. Untersuchungen zur Ventilrotation weisen einen signifikanten Einfluss auf den Ventilverschleiß nach [52], [139], [142]. Daneben lassen Untersuchungen zu den beiden Faktoren Zyklenanzahl (Lebensdauer) und Frequenz nicht eindeutige Trends erkennen. Wang et al. 1996 in [147] und Chun et al. 2007 in [142] schlussfolgern, dass sich der Verschleiß mit steigender Zyklenanzahl vergrößert, wobei die Frequenz gegenüber der Zyklenzahl überwiegt. Dem entgegen stehen die Studien von Mascarenhas et al. 2015 in [139]. Man konnte keinen Einfluss der Frequenz auf den Verschleiß feststellen.

Neben den mechanischen Belastungen Ventilschließen und Verbrennungsdruck ist ein weiterer Aspekt für den Ventilverschleiß von Wichtigkeit. Die Bildung von verschleißminimierenden Triboschichten kann Reibung und Aufschlagskräfte reduzieren. De Wilde schlussfolgerte bereits 1967 in [149] aus seinen Untersuchugen, dass ein Mikrogleiten zwischen den Kontaktflächen zu Hochverschleiß führt, wenn der Reibwert nicht durch die Bildung von Metalloxiden wie $\mathrm{FeO}$ und $\mathrm{Cr}_{2} \mathrm{O}_{3}$ reduziert werden kann. Weiterhin erkennt er eine Abhängigkeit zwischen Bildung von Metalloxiden, Werkstoffwahl und 
Betriebsbedingungen. Zhao et al. führten 1997 in [150] vergleichende Untersuchungen zum Verschleißverhalten verschiedener Werkstoffpaarungen bei $538^{\circ} \mathrm{C}$ und $649{ }^{\circ} \mathrm{C}$ durch. Die Autoren führten die beobachtete Abrasion auf Verschleißpartikel zurück, die sich aufgrund fehlender Koaxialität zwischen VS und SR sowie Gleitverschleiß gebildet hatten. Weiterhin wurde eine temperaturabhängige Korrelation von plastischer Deformation und Oxidation der Kontaktflächen geschlussfolgert. Wang et al. untersuchten 1998 in [151] mit dem gleichen Prüfstand wie [150] weitere Werkstoffkombinationen mit dem Ziel eines Rankings. Basierend auf den mikrostrukturellen Untersuchungen kamen die Autoren zur Aussage, dass Ventilverschleiß eine komplexe Kombination aus Adhäsion, Scherbelastungen und Abrasion ist, wobei die Bildung schützender Oxidfilme auf den Verschleißflächen entscheidend für die Lebensdauer ist. Liang et al. beobachteten 1999 in [152] neben der Entstehung und Ausbreitung von Rissen in der tribologisch beanspruchten Randzone unter Scherbelastung eine Oxidation der verschlissenen Oberflächen. Applikationsabhängig wurden die beiden Verschleißmechanismen Oberflächenzerrüttung für Gasmotoren und Tribooxidation für Dieselmotoren festgestellt, wobei die untersuchten intermetallischen Legierungen weniger Verschleiß aufgrund des kleineren Reibkoeffizienten aufwiesen, so die Autoren. Chun et al. analysierten 2007 in [142] die chemische Zusammensetzung von Triboschichten aus experimentellen Komponententests an verbrannter LPG-Atmosphäre. So wurden auf VS und SR neben O, C, S, V und Al jeweils Elemente des Gegenkörpers detektiert. In der Triboschicht der VS wurde $\mathrm{Cu}$ aufgrund Werkstoffübertragung vom SR nachgewiesen. Spätere Grundlagenuntersuchungen von Saunders et al. erweitern 2008 in [153] die Erkenntnisse von [142]. So hängt die Bildung von Oxiden auf Metallen und Legierungen signifikant von der umgebenden Gasatmosphäre und deren Wasserdampfgehalt ab. Karamangil et al. untersuchten 2008 in [154] den Einfluss unterschiedlich dicker kohlenstoffhaltiger Schichten auf Temperatur- und Spannungsverteilung im Ventilteller mittels numerischer Simulation. Man erkannte, dass mit steigender Dicke der kohlenstoffhaltigen Schichten Wärmeverluste, Ventiltemperaturen und Temperaturgradienten im Ventilteller sinken. Für eine zukünftig rußärmere Verbrennung schlussfolgerten die Autoren höhere Ventiltemperaturen. Mantey et al. beobachteten 2009 in [155], dass der Einsatz des Kraftstoffs LPG zu erhöhtem Verschleiß und vorzeitigem Funktionsausfall des Tribosystem VS/SR führt. Einen reduzierten Verschleiß bei Verwendung alternativer Werkstoffe erklärten die Autoren durch die Bildung von Oxidschichten auf den Kontaktflächen, wobei die Art der Oxide nicht näher beschrieben wurde. Studien von Forsberg et al. 2011 in [156] und 2013 in [12] fokussierten auf die Rolle von Triboschichten auf AVS und ASR für die Applikation Heavy-Duty. In [156] wurden aus verschiedenen Betriebsbedingungen desselben Motortyps zwei unterschiedliche Triboschichten auf typgleichen VSn nachgewiesen. Für die eine Triboschicht mit glatter Oberfläche wurde eine $5 \mu \mathrm{m}$ dicke Schicht aus Ca, O, P, S und Zn detektiert. Hingegen bestand die zweite Triboschicht mit rauerer Oberfläche aus einem zweilagig metallischen Oxidfilm. Die Probe mit metalloxidischer Triboschicht stammte aus einem Motor mit weniger Verbrennungsrückständen. Eine vergleichende Aussage zum Verschleiß der unterschiedlichen Triboschichten wurde nicht gemacht. In [12] wurden kompaktierte Triboschichten bestehend aus Rückständen verdampftem Motoröls, Metalloxiden und anderen Kohlenwasserstoffen auf 
VSn mit mildem Verschleiß nachgewiesen. Im Vergleich von VS und SR konnten dickere Triboschichten auf Seite der VS beobachtet werden. Lehmann und Renz untersuchten 2016 in [157] dimensionsgleiche VSn mit unterschiedlicher Panzerung am Ventilsitz aus demselben Großgasmotor mit schwerem (Stellite ${ }^{\mathrm{TM}}$ 12) und mildem (Tribaloy ${ }^{\mathrm{TM}}$ T400) Verschleiß. An der Oberfläche der VSn mit mildem Verschleiß aus Tribaloy ${ }^{\mathrm{TM}}$ T400 konnte ein metallischer Oxidfilm aus $\mathrm{Si}, \mathrm{Cr}$, Mo, $\mathrm{Zn}$ nachgewiesen werden.

Zusammenfassend sind aus dem Literaturstudium zwei relevante Aspekte festzuhalten. Zum einen sind Einflüsse und anteilige Zuordnungen der beiden Hauptparameter Ventilschließen und Verbrennungsdruck nicht hinreichend verstanden, zum anderen besteht ein signifikanter Einfluss von Triboschichten auf das Verschleißverhalten des Tribosystems VS/SR. In Bezug auf die Triboschicht werden drei Varianten deutlich; metallische Oxidfilme, kompaktierte Schichten aus Verbrennungsrückständen mit oder ohne Werkstoffübertragung und Schichtsysteme aus Mischformen bestehend aus Metalloxiden, Werkstoffübertragung und/oder Rückständen. Beim Belastungskollektiv gehen die Meinungen der Autoren hinsichtlich der eigenschaftsbestimmenden tribologischen Belastung auseinander. Einige betrachten den Stoßverschleiß infolge Ventilschließen als dominierend [137], [141], andere sehen den Gleitverschleiß als Folge des Verbrennungsdrucks als maßgebend an [144], [146]. Zusätzlich überlagern weitere Faktoren die tribologischen Vorgänge. Hier sind unzureichende Lagetoleranzen wie eine fehlende Koaxialität der Achsen von VS und SR, verschiedene Atmosphären, weniger Verbrennungsrückstände, temperaturinduzierte Effekte oder die Art der Verschleißpartikel zu nennen. Für den Aspekt Triboschicht darf festgestellt werden, dass wenn diese vorhanden ist, milder Verschleiß in allen Untersuchungen beobachtet werden konnte. Abbildung 1.17 veranschaulicht nach Ansicht des Autors drei Quellen für potenzielle Edukte zur Bildung einer Triboschicht. Erstens können teilweise verbrannte und unverbrannte Verbrennungsrückstände aus Kraftstoff und/oder Motoröl Ablagerungen auf den Kontaktflächen von VS und SR bilden [12]. Als zweite Quelle können Verschleißpartikel aus einer Werkstoffübertragung von VS oder SR resultieren [152], [151]. Drittens können tribochemische Reaktionen an den Kontaktflächen mit der Umgebungsatmosphäre zu Verschleißpartikeln führen [158], [155], [142], wobei insgesamt eine anteilige Zunahme von Oxidschichten mit steigender Temperatur angenommen werden darf. In Abhängigkeit von den beiden Faktoren Ventilfrequenz und Flächenpressung werden Verschleißpartikel unterschiedlich zu verschleißminimierenden Triboschichten kompaktiert.

Als Verschleißmechanismen wurden für den Ventilverschleiß alle vier Hauptmechanismen, Abrasion, Adhäsion, Oberflächenzerrüttung und tribochemische Reaktion, identifiziert [147], [159], [152], [156], [160]. Die Untersuchungen zum Einfluss der Temperatur auf das Verschleißverhalten des Tribosystems VS/SR verdeutlichen gemeinsam, dass steigende Temperaturen verschleißminimierend wirken [142], [147], [149], [151], [152] insbesondere oberhalb von $400^{\circ} \mathrm{C}$ [141]. Diese Ergebnisse werden durch eine erhöhte Bildung von metallischen Oxidfilmen erklärt. 


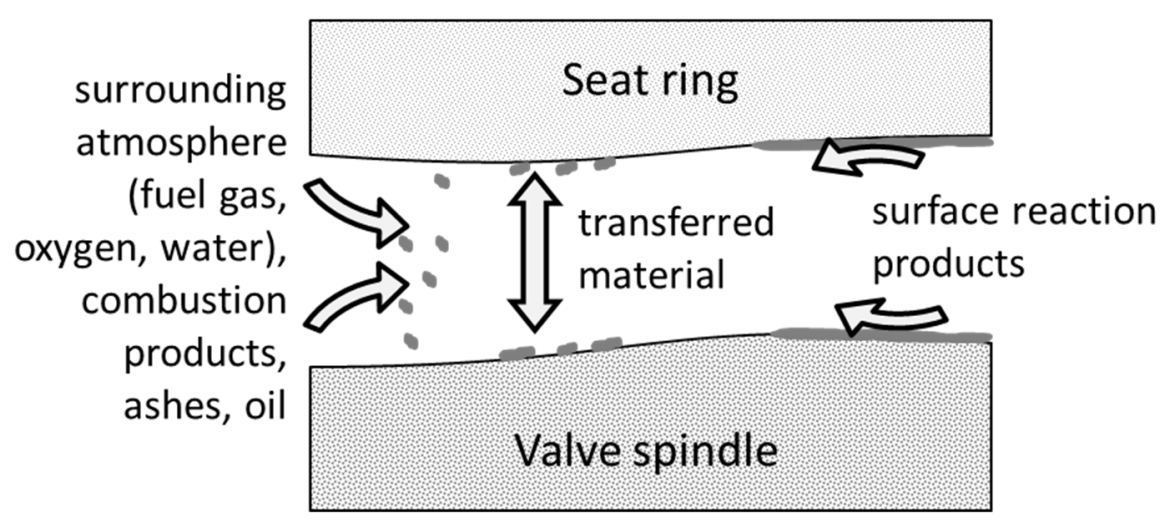

Abbildung 1.17: Quellen möglicher Edukte zur Bildung einer verschleißminimierenden Triboschicht auf den Sitzkontaktflächen von VS und SR nach [60]

\subsection{Ventilverschleißprüfstände}

Gaswechselventile in Pilzform werden seit über 120 Jahren in Verbrennungsmotoren eingesetzt [161]. In der wissenschaftlichen Literatur wird von sieben VVPen auf Komponentenebene berichtet. Davon können den Bereichen Automotive und Heavy-Duty fünf bzw. zwei zugeordnet werden. Verschleißprüfungen können nach Czichos [14] in sechs Kategorien unterteilt werden. Vom Komponenten- zum Modellversuch wird die Systemstruktur zunehmend mit dem Nachteil einer sinkenden Sicherheit der Ergebnisübertragbarkeit verändert. Der Modellversuch bietet messtechnisch eine bessere Zugänglichkeit zum Tribokontakt. Nach [143] und [162] sind Modellversuche zum Tribosystem VS/SR eingeschränkt aussagefähig.

Obwohl es in den letzten Jahrzehnten ein deutliches Interesse an dem Thema Ventilverschleiß gab [163], [164] und [165], war bis 1993 kein Komponentenprüfstand zur experimentellen Simulation des Ventilverschleißes wissenschaftlich dokumentiert. Eine erste Arbeit stellt Williams 1937 in [166] zur Untersuchung des Verschleißes von SRen im Bereich Automotive vor. Malatesta et al. führten 1993 in [138] einen auf Schadensfalluntersuchungen basierenden Prüfstand im Bereich Heavy-Duty ein, wobei man sich auf den mechanischen Verschleiß infolge Lagetoleranzen fokussierte. Die Prüfanordnung, welche später von Wang et al. 1996 in [147] weiterverwendet wurde, besitzt keine Ventilrotation und baut auf eine 2-säulige, servohydraulische Universalprüfmaschine mit feststehender VS auf. Der Einfluss von parallel versetzten und geschränkten VS- und SR-Achsen wurde abhängig von Temperatur und Verbrennungsdruck untersucht, wobei die Temperatur durch Verbrennung eines Propan-LuftGemischs erzeugt und mittels sechs Thermoelementen überwacht wurde. Die Versuche kombinierten Ventilschließen und Verbrennungsdruck. Als Verschleißkenngröße wurde die maximale Tiefe der Verschleißmarkenbreite definiert. Ein signifikanter Verschleiß wurde mit dem Erreichen einer Profiltiefe von 0,0254 mm oder größer nach $3 \times 10^{6}$ oder weniger Zyklen definiert. Die Prüfstandsvalidierung basierte auf einer vergleichenden Untersuchung der Verschleißerscheinungsformen von VSn aus Simulation und befeuerten Motortests. Angaben zu den Betriebsbedingungen von Prüfstand und Motortest sind nicht genannt worden. Verschleißraten wurden nicht verglichen. Lewis et al. stellen 1998 in [52] einen Prüfstand zur 
Simulation des Verbrennungsdrucks im Bereich Automotive vor. Der Prüfaufbau basiert auf einer servohydraulischen Universalprüfmaschine, wobei das Ventilöffnen mithilfe einer Feder erfolgte. Die Prüfanordnung erlaubt die Durchführung von Versuchen mit und ohne Entkopplung von VS und SR, wobei verschiedene Belastungsprofile (sinus- oder dreiecksförmige Lastkurven) gefahren werden können. Untersuchungen zum Verbrennungsdruck, Temperatur, Lastspielzahl, Koaxialität und Ventilrotation soll der Prüfstand ermöglichen. Mit der verwendeten Heißluftheizung können, wie später in [159] berichtet wird, Temperaturen bis zu $130{ }^{\circ} \mathrm{C}$ erreicht werden. Für die Prüfstandsvalidierung diente ein qualitativer Vergleich der Verschleißerscheinungsformen mit Teilen aus einem befeuerten Motorversuch nach 250 Betriebsstunden bei Motordrehzahl $4800 \mathrm{~min}^{-1}$ (entspricht $40 \mathrm{~Hz}$ Ventilfrequenz) zusammen mit Beschreibungen aus der Literatur. Nähere Angaben zur Prüffrequenz und Temperatur wurden nicht gemacht. Aus [159] darf eine Prüffrequenz von $10 \mathrm{~Hz}$ vermutet werden. Eine quantitative Verschleißbewertung in Form einer Profilometerie wurde für Teile aus dem Motortest durchgeführt. Die Autoren schlußfolgern aus den Verschleißerscheinungen, dass eine kombinierte Belastung aus Ventilschließen und Verbrennungsdruck eine verbesserte Simulation bieten würde als eine getrennte Simulation des Verbrennungsdrucks. Später erweiterten Lewis und Dwyer-Joyce 2002 in [159] die Versuchsanordnung um eine zweite elektrisch angetriebene Prüfstandseinheit zur Simulation des Ventilschließens, wobei die Schließgeschwindigkeit durch verschiedene Ventilspiele mittels unterschiedlich dicker Sitzringhalter variiert wurde. Der Prüfaufbau, bestehend aus einem Zylinderkopf eines Vierzylinder 1,8I-Dieselmotor montiert auf einem Stahlrahmen, wurde nicht validiert. Vielmehr wurden die Verschleißergebnisse aus den experimentellen Simulationen gleich zur Validierung von numerischen Simulationsergebnissen herangezogen. Bei Raumtemperatur wurde der Einfluss der Schließgeschwindigkeit auf den Ventilverschleiß über 160.000 Zyklen untersucht. Eine Antwort, inwieweit die Übertragbarkeit der bei Raumtemperatur durchgeführten Versuche auf den befeuerten Motorbetrieb zulässig ist, lassen die Autoren offen. Satish et al. beschreiben 2003 in einer Patentschrift [167] einen hydraulisch und/oder pneumatisch angetriebenen Prüfstand im Bereich Heavy-Duty mit getrennter Aktuatorik für Ventilschließen und Verbrennungsdruck. Weitere Informationen wurden in der wissenschaftlichen Literatur nicht publiziert. Chun et al. führen 2007 in [142] und [158] einen servohydraulischen Prüfstand auf Basis einer 2-säuligen Universalprüfmaschine zur Simulation von Temperatur, Frequenz, Zyklenanzahl, Verbrennungsdruck und Schließgeschwindigkeit im Bereich Heavy-Duty ein. Eine Ventilfeder dient zur Entkopplung von VS und SR. Mittels eines Propan-Luft-Gemisches kann die VS auf bis zu $900{ }^{\circ} \mathrm{C}$ erwärmt werden. Die genannten $760^{\circ} \mathrm{C}$ für den Ventilsitz wurden aus Literaturangaben von den Autoren abgeschätzt. Ventilschließen und Verbrennungsdruck wurden kombiniert simuliert. Nähere Angaben zur Ventilrotation wurden nicht gemacht. Als Verschleißkenngröße wurde der Rauheitswert Rmax definiert. Die vorgestellten Prüfstandsergebnisse wurden weder quantitativ in [142] noch qualitativ in [158] validiert. Motiviert durch die kosteneffektive Möglichkeit des Laserhärtens im Ventilsitzbereich eines Zylinderkopfs aus Grauguss als potenzielle Substitution von SRen stellen Slatter et al. 2009 in [143] einen Prüfstand im Bereich Automotive vor. Die Autoren betrachten das Ventilschließen 
als dominierenden Einflussfaktor. Der Prüfaufbau ermöglicht das gleichzeitige Testen von zwei VSn unter Variation von Schmierung, Schließgeschwindigkeit und Ventilspiel. Zur Reduktion der Versuchsanzahl gingen den Komponententests Modellversuche zum Stoßverschleiß mit dem Ziel eines Werkstoffrankings voraus. Als Verschleißkenngröße wurde der volumetrische Verschleiß definiert. Validiert wurde der Prüfstand durch einen Vergleich der Verschleißtiefen von Teilen aus einem befeuerten Motortest nach $1500 \mathrm{~h}$ und Prüfstandstest nach $100 \mathrm{~h}$ bei Raumtemperatur, wobei sich der Verschleiß an EVS und AVS umgekehrt darstellte; größte Verschleißtiefe an der EVS aus dem Motorversuch vs. größte Verschleißtiefe an der AVS aus dem Prüfstandsversuch. Nähere Angaben zur Prüffrequenz wurden nicht genannt. Die Autoren schlussfolgerten aus den unterschiedlichen Versuchsergebnissen, dass der Modellversuch zum Stoßverschleiß bedingt geeignet ist. Forsberg et al. führen 2012 in [168] einen servohydraulischen Prüfstand im Bereich Heavy-Duty ein. In [169] werden bis zu 25 kN Schließkraft für diese Prüfanordnung genannt. Im Versuchsbetrieb, so die Autoren, ist kein kombinierter Betrieb von Ventilrotation und Temperaturmessung an der VS möglich. Nähere Angaben, ob die Versuche mit oder ohne Ventilrotation bzw. Temperaturmessung erfolgten, wurden nicht gemacht. Aufgrund der Aussage in [169], dass die Temperaturmessung nur für Kalibrierungen eingesetzt wurde, darf eine Ventilrotation während der Versuche vermutet werden. Die maximale Prüffrequenz betrug $10 \mathrm{~Hz}$. Für die Validierung wurden als Kriterien ausbruchartige Verschleißerscheinungen am SAD, Werkstoffübertragung und die Bildung von Triboschichten herangezogen. Ausbrüche am SAD der VS und eine eisenhaltige Werkstoffübertragung vom SR auf die VS wurden für VSn aus Motor- und Prüfstandsversuch gleichermaßen beobachtet. Im Sitzbereich bestand die VS aus einer Stellite ${ }^{\mathrm{TM}}$ F-Panzerung und der SR aus Eisenbasis. Ferner konnte eine vergleichbare Triboschicht auf den VSn aus Prüfstand- und Motorversuch hinsichtlich Dicke, Zusamensetzung und Struktur nachgewiesen werden. Eine Verschleißrate wurden nicht gemessen. Mascarenhas et al. stellen 2015 in [139] einen durch das Phänomen Downsizing im Bereich Automotive motivierten Prüfstand zur realitätsnahen und zeitbeschleunigten Evaluation von Werkstoffen vor. Die auf einen Stahlrahmen aufbauende Prüfanordnung kann bis zu vier VS/SR-Paarungen gleichzeitig testen. Eine hydraulische Aktuatorik leitet über einen Druckstempel eine druckäquivalente Kraft auf die VS ein. Detaillierte Informationen zum Ventilschließen werden nicht genannt. Das Ventilöffnen wird mittels Ventilfedern realisiert. Für zeitbeschleunigte Untersuchungen wurde eine Nichtkoaxialität von Ventil- und Sitzringachse gewählt. Als Verschleißkenngrößen definierten die Autoren, wie Chun et al. in [142], die beiden Rauheitswerte Rmax und Rt, wobei die Größen gegenüber einem befeuerten Motortest nicht validiert wurden. Gleichsam wird das genannte Entwicklungsziel eines beschleunigten Verschleißtests für ein schnelles Werkstoffranking in der Publikation nicht validiert, vielmehr werfen die unterschiedlichen Versuchszeiten von $30 \mathrm{~h}$ für Experiment 1 und $10 \mathrm{~h}$ für Experiment 2 Fragen zur generellen Vergleichbarkeit der Testergebnisse auf. Darüber hinaus wurden die verwendeten Stellite ${ }^{\mathrm{TM}}$ gepanzerten Ventile aus SAE EV12 21-2N bei untypisch hoher Temperatur von $750{ }^{\circ} \mathrm{C}$ für austenitische Ventilstähle getestet. Tabelle 1.1 gibt eine Übersicht der dokumentierten VVPe auf Komponentenebene in der wissenschaftlichen Literatur bis 2015. 
Tabelle 1.1: Übersicht dokumentierter Ventilverschleißprüfstände in der wissenschaftlichen Literatur auf Komponentenebene bis 2015

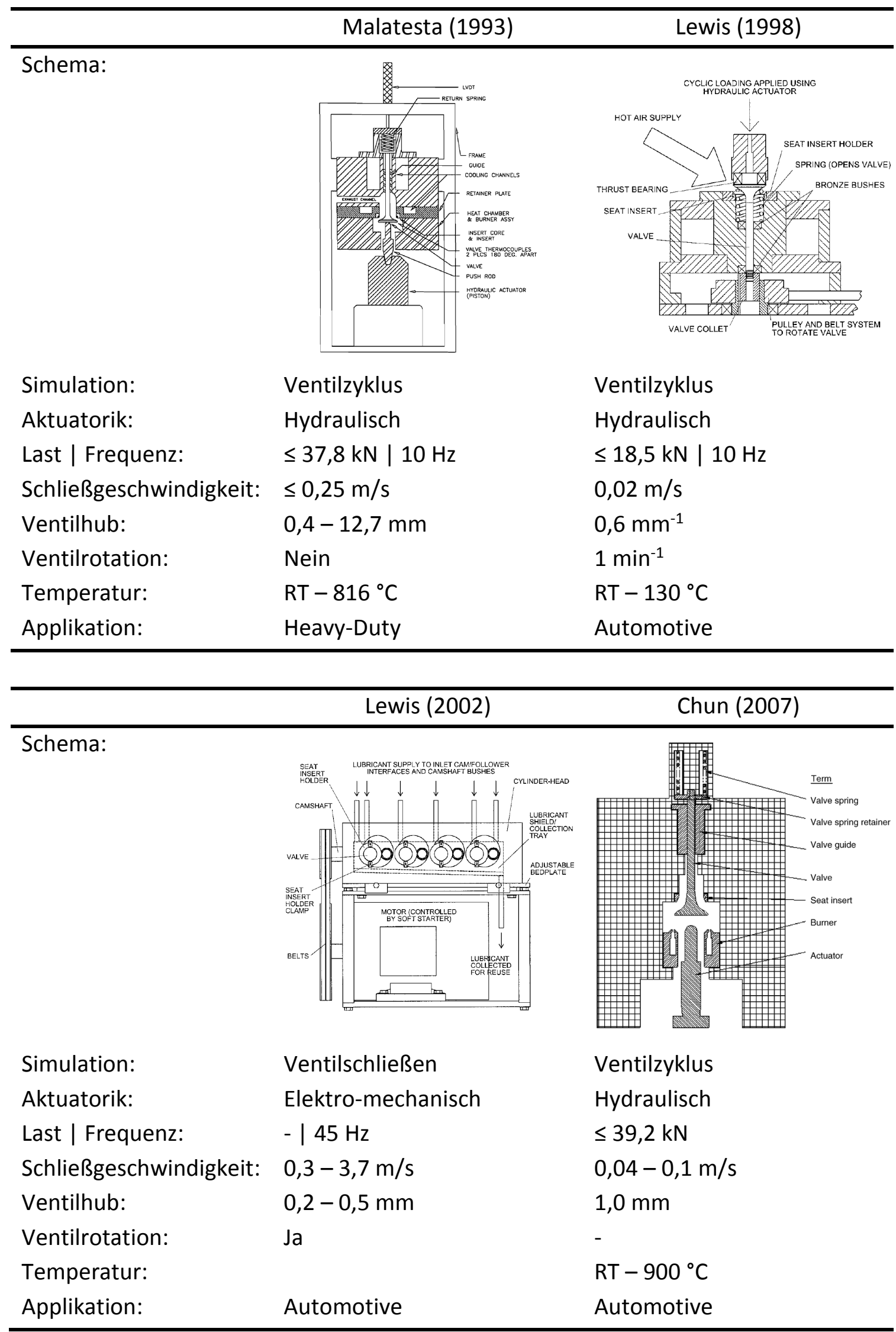




\begin{tabular}{l}
\hline Slatter (2009) \\
\hline Schema: \\
Simulation:
\end{tabular}

Mascarenhas (2015)

Schema:

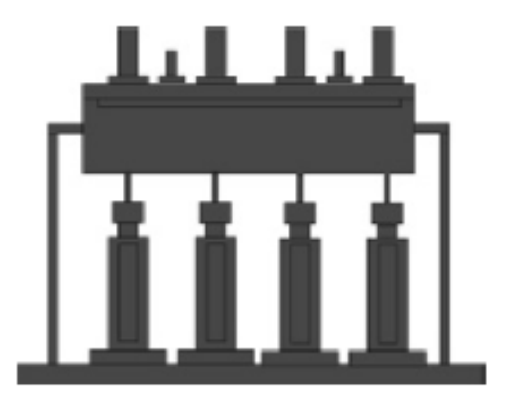

Simulation: Ventilzyklus

Aktuatorik: Hydraulisch

Last | Frequenz: $\quad 1-25 \mathrm{kN} \mid 50 \mathrm{~Hz}$

Schließgeschwindigkeit: $0,6 \mathrm{~m} / \mathrm{s}$

Ventilhub: $\quad 0,1-10 \mathrm{~mm}$

Ventilrotation: $\quad 4 \mathrm{~min}^{-1}$

Temperatur: $\quad \mathrm{RT}-900^{\circ} \mathrm{C}$

Applikation: Automotive 


\subsection{Zukünftige Trends in der Großgasmotorenentwicklung}

Zur besseren Einordnung dieser Arbeit soll in diesem Kapitel kurz auf zukünftige Trends in der Entwicklung von Großmotoren eingegangen werden, die im Wesentlichen von zwei Aspekten bestimmt wird. Einerseits gilt es den Wirkungsgrad weiter zu verbessern, andererseits sind die gesetzlichen Emissionsvorgaben zu erfüllen. Zukünftig sind höhere Ladedrücke von $\geq 12$ bar [111] im Verbund mit optimierten Ventilsteuerzeiten wie Miller-Valve-Timing [120] zu erwarten. Daneben ist mit einem zunehmenden Einsatz von variablen Ventilsteuersystemen vor dem Hintergrund einer steigenden Applikationsdiversifizierung zu rechnen [170]. Derzeitig wird das erreichbare Wirkungsgradpotenzial von Großgasmotoren auf ca. $60 \%$ geschätzt [171], wobei in den nächsten Jahren durch verbesserte brennverfahrensseitige Maßnahmen Zünddrücke von 300 bar zu erwarten sind [172]. Zur Einhaltung der Abgasgesetzgebung werden neben optimierten Verbrennungsprozessen die Abgasnachbehandlung [120], die Hybridisierung des Antriebs [173] und alternative Brennstoffe wie Wasserstoff [174] eine wichtige Rolle spielen. Parallel ist anzunehmen, dass an der Verlustschmierung über Kolben und insbesondere Ventilschaft weitergearbeitet wird. Im Vergleich zum Dieselmotor wird der Gasmotor aufgrund der geringeren Rohemissionen zukünftig an Bedeutung gewinnen. Aus den genannten Maßnahmen zur Wirkungsgraderhöhung leiten sind höhere thermische, mechanische und chemische Belastungen für das Tribosystem VS/SR ab. Auf der anderen Seite wird eine weitere Emissionsreduzierung insbesondere der Ruß- und Feinstaubpartikel zu weniger Verbrennungsrückständen führen, die zur Bildung verschleißminimierender Triboschichten auf den Kontaktflächen von VS und SR zur Verfügung stehen.

Im Zeitalter der Energiewende entstehen neue unterschiedliche Anforderungen an Großmotoren. Bislang waren Großmotoren auf Dauerbetrieb ausgelegt. Der Einsatz im hybriden Betrieb mit erneuerbaren Energien wie Windkraft und Photovoltaik führt zu neuen Lastprofilen und Betriebsverhalten mit hunderten Ein- und Ausschaltvorgängen pro Jahr [11]. Neben dem Grundlastbetrieb gewinnen schnelles Hochfahren zum Abdecken kurzfristiger Bedarfe bei Ausfall von Sonnen- oder Windenergie an Bedeutung. Zusammenfassend darf festgehalten werden:

(1) Erhöhung der Turboladerdrücke auf $\geq 12$ bar,

(2) steigende Zünddrücke von $\geq 300$ bar,

(3) optimierte Verbrennungsprozesse $\rightarrow$ minimierte Verbrennungsrückstände,

(4) reduzierte Verlustschmierung über Ventilschaft,

(5) Diversifizierug der Applikationen Marine, Lokomotive und Kraftwerk,

(6) parallele Entwicklung von Diesel- und Gasmotor,

(7) zunehmende Bedeutung des Gasmotors,

(8) Einsatz und Entwicklung von Abgasnachbehandlungssystemen,

(9) Hybridisierung des Antriebs und

(10) neue Lastprofile mit hunderten Ein- und Ausschaltvorgängen pro Jahr. 


\subsection{Ziel und Ansatz der Arbeit}

Das übergeordnete Ziel dieser Dissertation ist durch Entwicklung eines VVPs ein verbessertes Verständnis über das Tribosystems VS/SR in hocheffizienten Großgasmotoren während der Phasen Ventilschließen und Verbrennungsdruck zu erlangen.

Für eine systematische Untersuchung der tribologischen Grundlagen zur Identifikation der eigenschaftsbestimmenden Verschleißmechanismen versucht diese Arbeit einen neuen VVP auf Komponentenebene zu entwickeln. Die Wirkmechanismen für Schließen und Zünddruck sollen in separaten Prüfanordnungen untersucht und quantitativ bewertet werden können. Der VVP soll dazu befähigen die tribologisch dominierenden Verschleißerscheinungsformen und typischen Verschleißraten, wie sie aus Motorversuchen bekannt sind, zu simulieren.

In der wissenschaftlichen Literatur sind nur Ventilprüfstände auf Komponentenebene für die Applikationen Automotive und Heavy-Duty dokumentiert. Einzig der aus dem Patent von [167] bekannte VVP könnte die Fähigkeit besitzen, großmotorische VSn tribologisch zu untersuchen. Als häufige Herangehensweise für die Entwicklung der literaturbekannten Prüfstände wurde entschieden, die zu untersuchenden Parameter so realistisch wie möglich zu simulieren [52], [138], [139], [167]. Die zum Großteil auf Universalprüfmaschinen mit servohydraulischen Antrieb basierenden Prüfaufbauten hatten alle das Ziel den Einfluss von potenziellen Einflussfaktoren auf den Ventilverschleiß zu untersuchen, wobei nicht alle Prüfstände validiert wurden, vielmehr hatten die Versuche oftmals das Ziel eines Werkstoffrankings [151], [139], [143], [158]. Zur Validierung dienten qualitative Vergleiche der Verschleißerscheinungen und quantitative Messungen von Verschleißmarkenbreite oder Rauheit im Bereich der Sitzkontaktfläche [158], [52], [143], [175]. Keiner der Prüfstände wurde einer kombinierten Validierung von Verschleißerscheinungsformen und Verschleißrate unterzogen. Vereinzelt wurden Bauteilversuchen Modellversuche mit nicht übertragbaren Ergebnissen vorangestellt [143] oder zeitbeschleunigte Versuche mittels höherer Prüffrequenzen ohne Abgleich mit dem befeuerten Motorbetrieb durchgeführt [139], wodurch die Korrelationsprüfungen innerhalb der tribologischen Prüfkette verletzt wurden [14]. Bis auf [52] und [143] wurde das Phänomen Ventilverschleiß stets als kombinierter Ventilzyklus der beiden Phasen Schließen und Verbrennungsdruck untersucht. Beide Autoren untersuchten für die Applikation Automotive das Ventilschließen ohne die Ergebnisse mit Teilen aus einem befeuerten Motorbetrieb zu vergleichen. Die Verschleißkenngröße Verschleißrate mit Werkstoffabtrag pro Zeiteinheit wurde in keiner Publikation zum Thema Ventilverschleiß angewendet, wobei gerade diese eine geeignete Vergleichbarkeit zwischen Prüfstand- und Motorversuch schafft. Inwieweit die gemachten Aussagen aus Prüfstandsversuchen ohne kombinierte Validierung von Verschleißerscheinungsformen und Verschleißrate auf den Betriebsversuch übertragbar sind, ist fraglich. Daher wird als methodischer Ansatz zur Entwicklung eines neuen VVPs für großmotorische Applikationen für diese Arbeit im ersten Schritt eine mikrostrukturelle Untersuchung der tribologisch beanspruchten Randschicht von VSn aus dem Feld gewählt, um die eigenschaftsbestimmenden Verschleißmechanismen des Tribosystems VS/SR in hocheffizienten Großgasmotoren zu identifizieren. Im zweiten Schritt wird abhängig von den 
Erkenntnissen aus der Mikrostrukturuntersuchung in Verbindung mit dem Stand der Wissenschaft und Technik über VVPe das Konzept für den zu entwickelnden VVP abgeleitet. Als dritter Schritt folgen Entwicklung, Konstruktion und Aufbau eines neuartigen VVPs mit getrennter Simulation der beiden Phasen Ventilschließen und Verbrennungsdruck für Großgasmotoren. Mittels getrennter Simulation der verschleißrelevanten Phasen soll ein verbessertes Verständnis der dominierenden Verschleißmechanismen in den Einzelphasen erreicht werden. Im letzten Schritt wird der neuentwickelte VVP validiert und erste Ergebnisse vorgestellt. Die beiden Kenngrößen Verschleißerscheinungsformen und Verschleißrate stehen dabei im Fokus. Ziel dieser Arbeit ist es, einen VVP zu entwickeln, der die eigenschaftsbestimmenden Verschleißmechanismen nachbilden und vergleichbar hohe Verschleißraten in Verbindung mit typischen Verschleißerscheinungen, wie sie aus dem Motorversuch bekannt sind, erzeugen kann. 


\section{Untersuchung der Verschleißmechanismen zur Prüfstandsentwicklung}

\subsection{Probenmaterial}

Die Untersuchungen wurden an insgesamt vier typgleichen VSn aus dem Feld mit unterschiedlicher Laufzeit und Verschleiß durchgeführt. Von vergleichbar ähnlichen Belastungskollektiven darf ausgegangen werden, da die vier dimensionsgleichen Proben aus demselben Großgasmotor stammten. Tabelle 2.1 gibt eine Übersicht von Probenbezeichnung, Panzerung im Sitzbereich, Position und Betriebsstundenanzahl. Es sei angemerkt, dass die Proben A3 und A4 zeitgleich im selben Zylinderkopf liefen.

Tabelle 2.1: Übersicht der untersuchten Proben

\begin{tabular}{ccccc}
\hline Probe & Komponente & Panzerung & Position & Betriebsstunden \\
\hline A1 & VS & Stellite $^{\mathrm{TM}} 12$ & Einlass & $3 \mathrm{~h}$ \\
A2 & VS & Stellite $^{\mathrm{TM}} 12$ & Einlass & $30 \mathrm{~h}$ \\
A3 & VS & Stellite $^{\mathrm{TM}} 12$ & Einlass & $250 \mathrm{~h}$ \\
A4 & VS & Stellite $^{\mathrm{TM}} 12$ & Auslass & $250 \mathrm{~h}$ \\
\hline
\end{tabular}

Die Auswahl der Proben erfolgte mit dem Ansatz, neben einem möglichen Einlaufverhalten die Verschleißentwicklung über die Zeit und die Ursachen der bis dato unbekannt großen Verschleißraten von bis $\mathrm{zu} 11,8 \mathrm{~mm}^{3} / \mathrm{h}$ zu verstehen. In Tabelle 2.2 ist die chemische Zusammensetzung der PTA-auftraggeschweißten Co-Basislegierung Stellite ${ }^{\text {TM }} 12$ genannt.

Tabelle 2.2: Nominell chemische Zusammensetzung von Stellite ${ }^{\mathrm{TM}} 12$ in Gew.-\%

\begin{tabular}{lcccccccc}
\hline & $\mathbf{C}$ & $\mathbf{S i}$ & $\mathbf{M o}$ & $\mathbf{C r}$ & $\mathbf{N i}$ & $\mathbf{W}$ & $\mathbf{F e}$ & Co \\
\hline Stellite $^{\mathrm{TM}} 12$ & 1,6 & 1,2 & - & 28,0 & $<3,0$ & 8,0 & $<1,0$ & Bal. \\
& - & - & & - & & - & & \\
& 1,8 & 1,7 & & 32,0 & & 10,0 & & \\
\hline
\end{tabular}

In der industriellen Praxis wird der Ventilverschleiß als Ventilrückgang in überwiegender Zahl mittels Tiefenmaß gemessen. Dies entspricht streng genommen dem Systemverschleiß von VS und SR. Bei den Proben A1 bis A4 darf versichert werden, daß der gemessene Ventilrückgang nahezu dem Komponentenverschleiß der VS entsprach. Damit stellt die VS für diese Untersuchung das bestimmende Element dar. Die zugehörigen Gegenkörper standen für eine Untersuchung nicht zur Verfügung. Bei den Proben A1 bis A4 handelt es sich um reibgeschweißte Bimetall-VSn aus dem aufgestickten austenitischen Nickel-Chrom-ManganStahl X50CrMnNiNbN21-9 (1.4882) im Bereich des Ventiltellers und dem martensitischen Ventilstahl X45CrSi9-3 (1.4718) im Bereich des Ventilschafts. Tabelle 2.3 gibt die chemischen Zusammensetzungen wieder. 
Tabelle 2.3: Nominell chemische Zusammensetzungen der Ventilwerkstoffe in Gew.-\%

\begin{tabular}{|c|c|c|c|c|c|c|c|c|}
\hline & C & $M n$ & $\mathrm{Cr}$ & $\mathrm{Ni}$ & Nb & Si & $\mathbf{N}$ & $\mathrm{Fe}$ \\
\hline \multirow[t]{3}{*}{1.4882} & 0,45 & 8,0 & 20,0 & 3,5 & 1,8 & $<0,5$ & 0,4 & Bal. \\
\hline & - & - & - & - & - & & - & \\
\hline & 0,55 & 10,0 & 22,0 & 5,0 & 2,5 & & 0,6 & \\
\hline \multirow[t]{3}{*}{1.4718} & 0,40 & $<0,6$ & 8,5 & $<0,5$ & - & 2,7 & - & Bal. \\
\hline & - & & - & & & - & & \\
\hline & 0,50 & & 10,0 & & & 3,3 & & \\
\hline
\end{tabular}

In Abbildung 2.1 ist die typische Gefügeausbildung einer PTA-Auftragschweißung von Stellite $^{\mathrm{TM}} 12$ dargestellt. Charakteristisch für die auftraggechweißte Hartlegierung ist eine untereutketische Erstarrung mit dendritischer Struktur aus $\mathrm{Cr}$ - und W-Karbiden (dunkle Phase) eingebettet in metallischen Zellen aus Co-Cr-Mischkristall (helle Phase), wobei in Abhängigkeit vom Abkühlungsgradienten Sigma-Phasen (weiße Phasen) auftreten können. Der tribotechnische Werkstoff Stellite ${ }^{\mathrm{TM}} 12$ wird in großer Zahl am Ventilsitz eingesetzt.

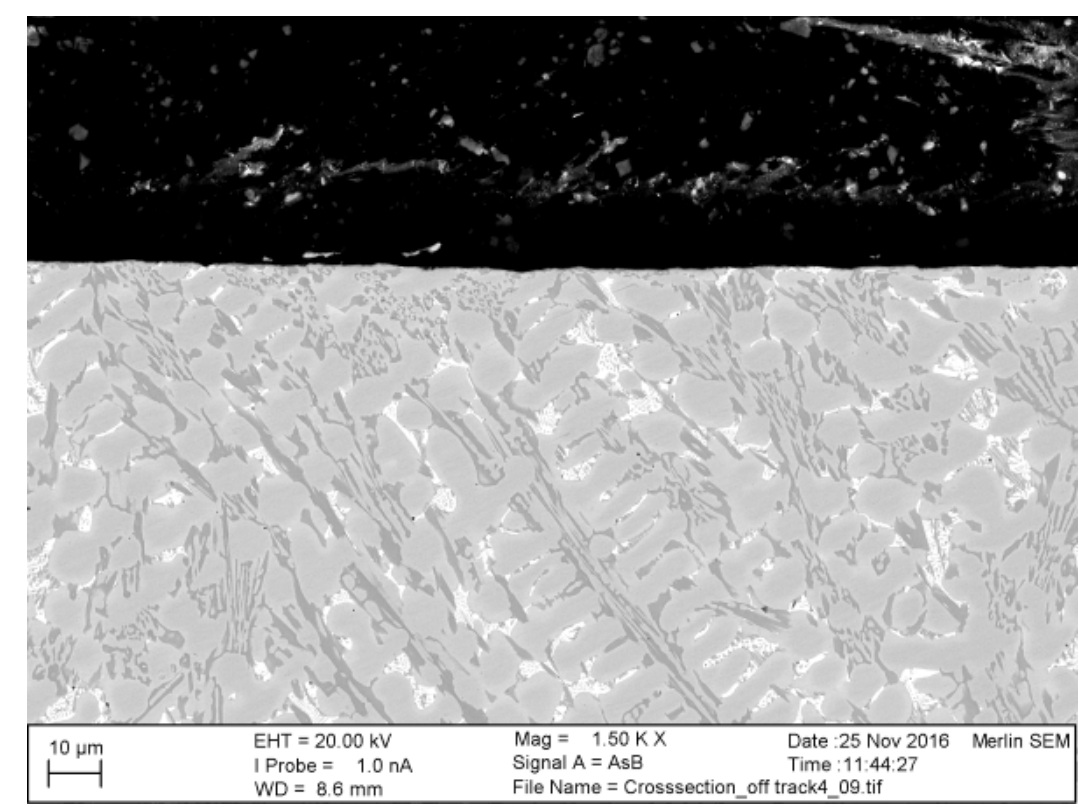

Abbildung 2.1: Typisches Gefüge einer PTA-Auftragschweißung von Stellite ${ }^{\mathrm{TM}} 12$; Vergrößerung 1500fach

In Tabelle 2.4 ist die chemische Zusammensetzung des schleudergegossenen SR-Werkstoffs Pleuco $12 \mathrm{MV}$ für die ersten Prüfstandsversuche wiedergegeben.

Tabelle 2.4: Nominell chemische Zusammensetzung von Pleuco $12 \mathrm{MV}$ in Gew.-\%

\begin{tabular}{lccccccc}
\hline & $\mathbf{C}$ & $\mathrm{Si}$ & $\mathrm{Mn}$ & $\mathrm{Cr}$ & $\mathbf{N i}$ & $\mathrm{Mo}$ & $\mathrm{Fe}$ \\
\hline Pleuco 12 MV & 1,8 & 0,8 & $\leq 0,6$ & 12,0 & $\leq 0,5$ & 2,0 & Bal. \\
& - & - & & - & & - & \\
& 2,3 & 1,0 & & 14,0 & & 2,5 & \\
\hline
\end{tabular}




\subsection{Mikrostrukturanalyse}

\subsubsection{Durchführung und Mikroskopie}

Für die mikroskopischen Untersuchungen von Verschleißspur in Draufsicht und tribologisch beanspruchter Randschicht im Querschliff kam ein Lichtmikroskop vom Typ Eclipse E 600 der Firma Nikon Instruments Inc. sowie ein Rasterelektronenmikroskop vom Typ Supra 40 VP der Firma Carl Zeiss Microscopy GmbH zur Anwendung. Zur Charakterisierung im REM war es im ersten Schritt erforderlich die VSn aufgrund ihrer Bauteilgröße im Übergang Hohlkehle/Schaft mittels Trennschleifen zu teilen. Da beim Trennschleifen Kontaminationen in die Oberfläche infolge des Kühlschmierstoffs eingetragen werden, erfolgte die Probenentnahme im nächsten Arbeitsschritt durch Heraustrennen einer ca. $5 \mathrm{~mm}$ dicken Probe mittels Drahterodieren. In Abbildung 2.2 ist die Probenentnahme mit drei markierten Untersuchungsbereichen am SID, SM und SAD an einer VS für die Oberflächen- und Mikrostrukturanalyse dargestellt. Anschließend wurden die erodierten Proben nach dem Schleifen mit 1200er SiCNassschleifpapier in einem mehrstufigen Polierprozess beginnend mit 9 über 6, 3 und $1 \mu \mathrm{m}$ Diamantsuspension fertigpräpariert. Ausgewählte Proben wurden zur Sichtbarmachung von Scherbändern geätzt. Die Ätzprozedur wurde als spannungsloses Wischätzen (kein potentiostatisches Ätzen) mit einer Lösung aus $100 \mathrm{ml}$ destilliertem Wasser, $10 \mathrm{ml}$ Salpetersäure (65 \%), 50 ml Salzsäure (32\%) und 10 g Eisen(III)-Chlorid durchgeführt. Hierbei wurde die Probe mit einem in der Ätzlösung getränkten Wattestäbchen bestrichen, mittels Lichtmikroskop kontrolliert und bis zum gewünschten Ätzgrad wiederholt behandelt, wobei die Schliffe zwischendurch mit warmen Leitungswasser abgespült wurden. Nach dem Ätzen erfolgte eine Probenreinigung mittels Ethanol im Ultraschallbad. Proben für REMUntersuchungen wurden final zur Vermeidung von Aufladungen mit Platin besputtert. Die Prozessdauer je Probe betrug ca. $20 \mathrm{~min}$. Proben für eine weiterführende Röntgenphotoelektronenspektroskopie wurden vor dem Einschleusen nur mit Ethanol gereinigt und bei Heißluft getrocknet.

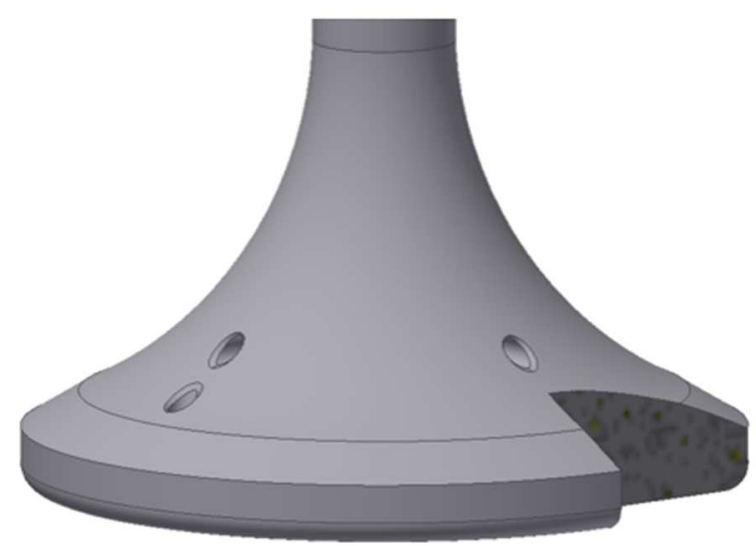

(a)

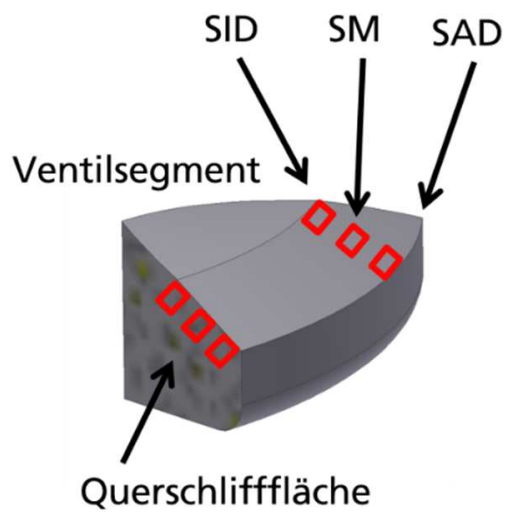

(b)

Abbildung 2.2: Probenentnahme, (a) schaftgetrennte VS mit erodierter Probe, (b) Ventilsegment mit drei markierten Untersuchungsbereichen am SID, SM und SAD 


\subsubsection{Spektroskopie}

Für das bessere Verständnis eines Tribosystems ist die Bestimmung der chemischen Zusammensetzung in der tribologisch beanspruchten Randschicht ein relevantes Werkzeug. Aus den Informationen zur Chemie von Mikrostruktur und Triboschicht lassen sich wichtige Erkenntnisse zu den eigenschaftsbestimmenden Verschleißmechanismen ableiten. Zur quantitativen Analyse der metallischen Elemente wurde die energiedispersive Röntgenspektroskopie (EDS) verwendet. Für die EDS kam ein Detektor des Typs Silizium Drift X-MAX 50 der Firma Oxford Instruments zur Anwendung. Die verfahrensbedingte Unschärfe der EDS-Analyse aufgrund der einerseits hohen erforderlichen Anregungsspannung von 10 bis 20 kV mit nur wenig emittierter Röntgenenergie der leichten Elemente, welche zum Großteil von den schweren Elementen wie $\mathrm{W}$ und $\mathrm{Cr}$ in den Hartlegierungen absorbiert wird und dem großen Wechselwirkungsvolumen (Anregungsbirne) aufgrund der Strahlintensität des fokussierten Primärelektronenstrahls, wurde die Elementanalyse um die hochauflösende Röntgenphotoelektronenspektroskopie (XPS) erweitert. Die Erfassung von chemischen Veränderungen mit hoher Empfindlichkeit und Tiefenauflösung in der Mikrostruktur mittels XPS schafft neben der quantitativen Bestimmung aller Elemente, außer Helium und Wasserstoff, die Möglichkeit zur Tiefenanalyse von Sauerstoff. Für die XPS wurde ein Spektrometer des Typs PHI 5000 VersaProbe der Firma ULVAC-PHI verwendet. Die Oberflächenchemie wird auf Basis des äußeren photoelektrischen Effekts bestimmt. Die verwendete Strahlungsquelle erzeugt vorzugsweise Röntgenstrahlung des Typs Mg-K $\alpha$ - oder Al-K $\alpha$ (1253,6 eV bzw. 1486,6 eV) und regt somit die Rumpfelektronen der inneren Schalen eines Atoms an. Als Messergebnis werden die charakteristischen Röntgenlinien der emittierten Photoelektronen ohne Energieverlust und deren freie Weglängen (energieabhängig), die im Bereich weniger $\mathrm{nm}$ liegen, analysiert. Abhängig von der Anregungsenergie beträgt die Informationstiefe ca. 0,5 bis $5 \mathrm{~nm}$ [176], d.h. wenige Atomlagen. Als Leistungsgrenze sei eine elementabhängige Nachweisgrenze von 0,1 bis 1 at.- \% genannt. Für die Tiefenprofilanalyse der Proben wurde ein Röntgenstrahl mit einer Spotgröße von $200 \mu \mathrm{m}$ verwendet, wobei eine Fläche von $2 \times 2 \mathrm{~mm}$ mit einem $3 \mathrm{kV}$ Argonionenstrahl gerastert wurde. Der Strahlstrom betrug 2500 bis $4000 \mathrm{nA}$, um eine Sputterrate von 10 bis $20 \mathrm{~nm} / \mathrm{min}$ basierend auf dem $\mathrm{SiO}_{2}$-Standard zu erreichen. Nach jedem Sputterstep wurde eine XPS-Messung durchgeführt. Für die Oberflächenreinigung wurde ein $3 \mathrm{kV} 2500 \mathrm{nA}$ Argonstrahl als lonensputter eingesetzt. Zur Vergleichbarkeit der Messergebnisse wurden die Messpositionen an den verschlissenen Oberflächen möglichst an derselben Position im Bereich des SADs durchgeführt. Die Tiefenprofilanalysen für Kohlenstoff werden gemischt als $\mathrm{C} / \mathrm{CHx}$ (Kohlenstoff / Kohlenwasserstoff) dargestellt, da nicht zwischen gebundenem (karbidisch) und ungebundenem (Ruß) Kohlenstoff unterschieden werden kann. 


\subsubsection{Profilometrie}

Zur Bestimmung der Kenngröße Verschleißrate wurde die taktile und optische Profilometrie eingesetzt. Die Anwendung des taktilen Tastschnittverfahrens erfolgte für die Proben A1 bis A4 sowie anfangs für die Proben der experimentellen Simulation. Als Messstart für alle Proben wurde ein Teilkreis mit einem radialen Abstand von $1 \mathrm{~mm}$ zum SID in Richtung Ventilachse definiert. Für die taktile Verschleißprofilmessung wurde ein Profilometer vom Typ HommelEtamic T800 Contour der Firma Jenoptik Group AG verwendet, wobei eine Nachbearbeitung der Profildaten aufgrund der hohen Profilschriebqualität nicht erforderlich war. Die taktile Profilmessung ist an demontierten Proben gut durchführbar. In der Versuchspraxis hingegen war diese Art der Messung mit einem erheblichen Zeitaufwand wegen Demontage und Montage der VS verbunden. Ferner stellt ein wiederholter Aus- und Zusammenbau für die Profilmessung neben einer möglichen Fehlerquelle für die weitere Versuchsdurchführung einen vermeidbaren Eingriff in das laufende Tribosystem VS/SR dar, wodurch sich unterschiedliche Ausgangszustände einstellen könnten. Daher wurde im weiteren Verlauf der Versuchsdurchführung nach fünf Vergleichsmessungen an derselben VS ohne signifikantem Unterschied auf die optische Profilometrie gewechselt. Für die optische Erfassung der Verschleißprofile wurde von den Kontaktflächen ein Negativabdruck mit einer Abdruckmasse vom Typ Reprint 2000 Base der Firma Cloeren Technology GmbH 2000 genommen. Das generierte Negativprofil wurde zur Auswertung mit einem konfokalem 3DLaserscanningmikroskop vom Typ VK-9710K der Firma Keyence in ein Segment geschnitten und anschließend eine Profilmessung durchgeführt. Zur Vermeidung von Peaks im Verschleißprofil infolge von Verunreinigungen oder anderen Messartefakten wurde das weiterverwendete Profil aus 100 Einzelmessungen gemittelt. Abbildung 2.3 veranschaulicht die Methodik zur Bestimmung der verschlissenen Fläche $A_{I, x h}$. Die Differenz aus der Fläche im Neuzustand $A_{I, 0 h}$ und der verschlissenen Fläche nach x-Stunden $A_{I, x h}$ ergibt die Verschleißfläche $A_{W, x h}$. Das Profil bildet eine geschlossene Kurve. Somit entspricht das Umlaufintegral dem Verschleißvolumen $V_{W, x h}$. Der Quotient aus Verschleißvolumen $V_{W, x h}$ und Zeit $\mathrm{t}$ ist definitionsgemäß die Verschleißrate W. Nach 0, 10, 25, 40, 55, 70 und $100 \mathrm{~h}$ wurde das Verschleißprofil an der VS erfasst. Aufgrund der Einbausituation vom SR wurde dessen Profil am Anfang und am Ende eines Versuchs gemessen.

$$
\begin{gathered}
A_{W, x h}=A_{I, 0 h}-A_{I, x h} \\
\oiiint d V_{W, x h}=\int_{0}^{2 \pi}(A \times r) d \varphi=A_{W, x h} \times U=A_{W, x h} \times 2 \times \pi \times r
\end{gathered}
$$

mit

$$
r=\frac{r_{a}+r_{i}}{2}
$$




$$
W=\frac{V_{W, x h}}{t}
$$

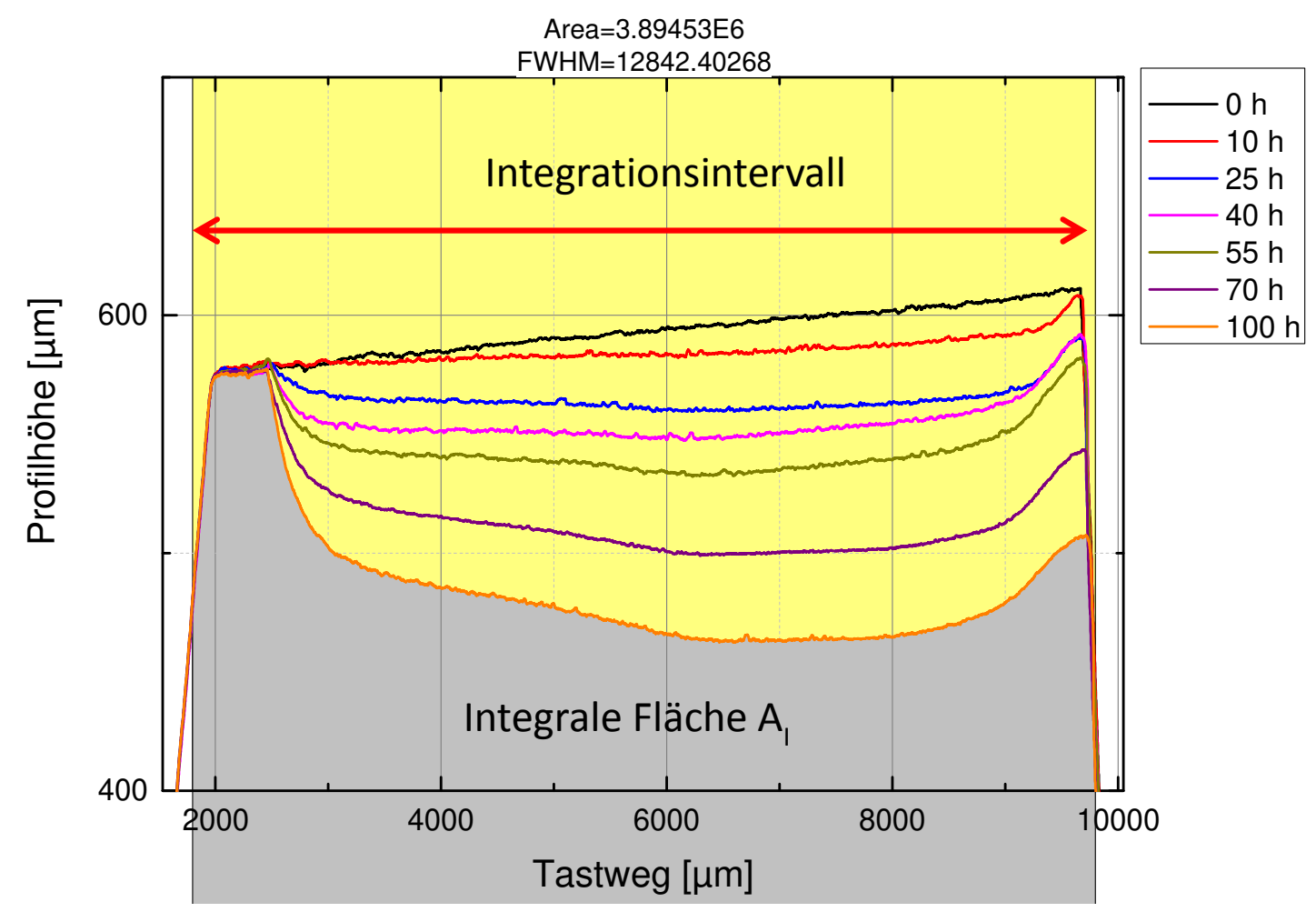

Abbildung 2.3: Bestimmung der verschlissenen Fläche $A_{I, x h}$ 


\subsection{Stellite ${ }^{\mathrm{Tm}} 12$-Ventilspindeln mit Verschleißraten von bis zu $11,81 \mathrm{~mm}^{3} / \mathrm{h}$}

\subsubsection{Mikroskopie}

Abbildung 2.4 veranschaulicht die schaftgetrennten Ventilteller der Proben A1 bis A4 nach der Probenentnahme. Man beobachtet auf den Makroaufnahmen der Proben A1 bis A3 laufzeitabhängige Ablagerungen von ölkohlehaltigen Verbrennungsrückständen im Bereich der Hohlkehle. Hingegen finden sich an der Probe A4 nach 250 Betriebsstunden nahezu keine Verbrennungsrückstände, vielmehr ist eine angelaufene, korrodierte Oberfläche im Bereich der Hohlkelhe zu erkennen.

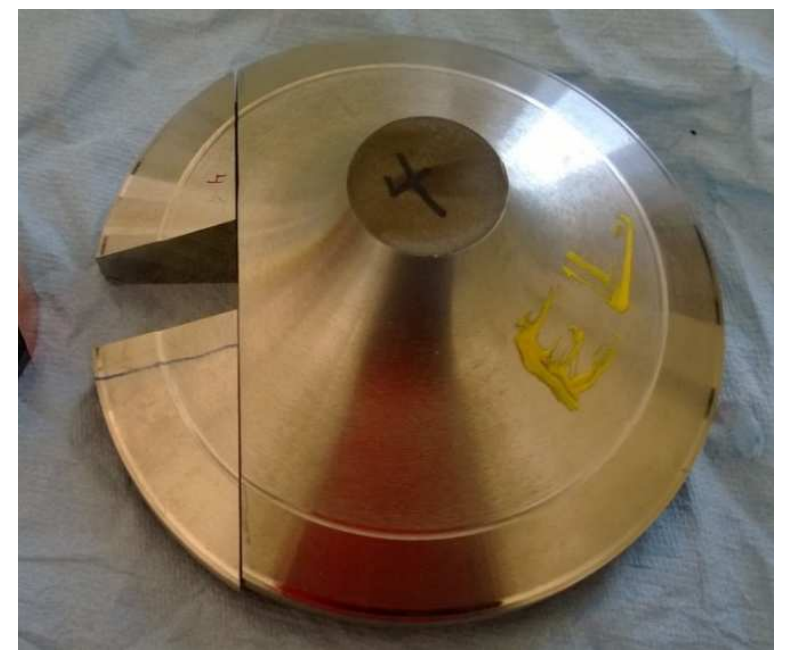

(a) A1, 3 Betriebsstunden

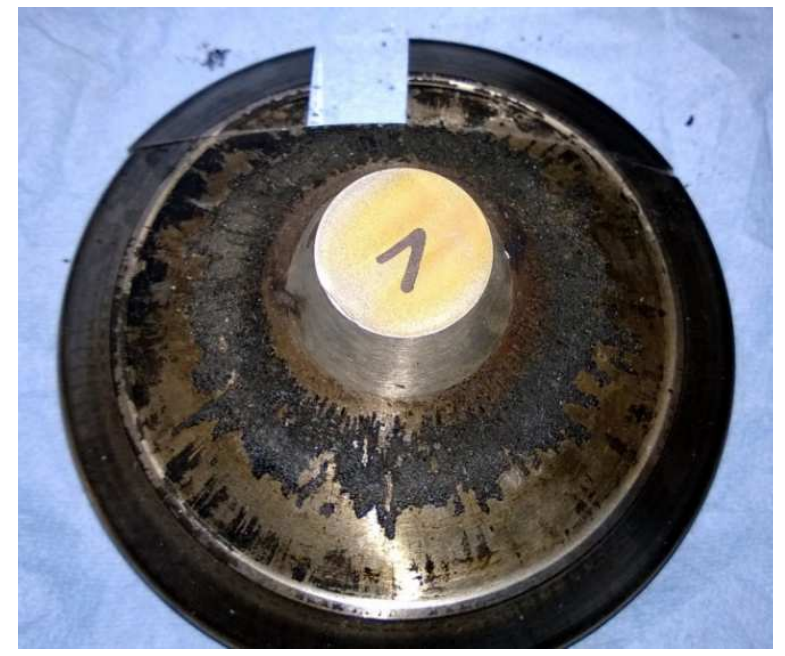

(c) A4, 250 Betriebsstunden

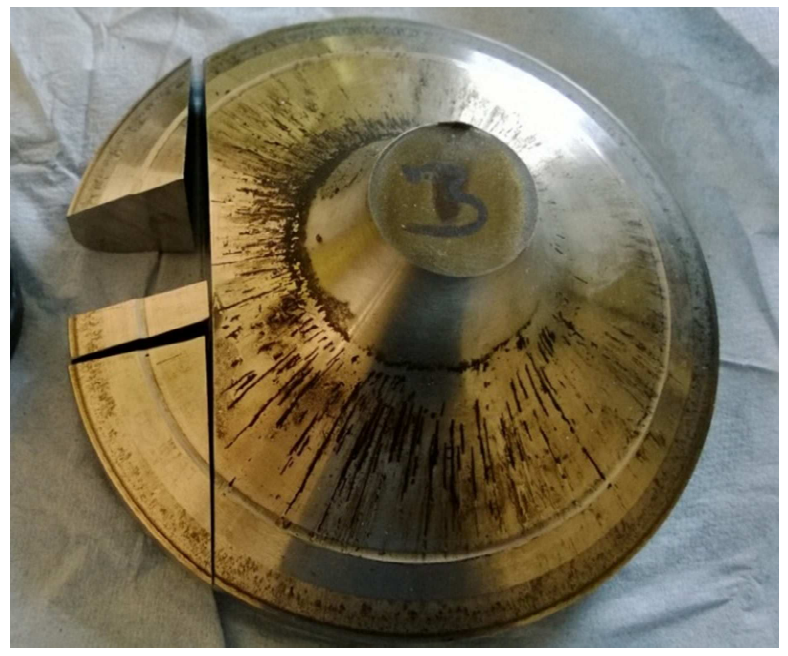

(b) A2, 30 Betriebsstunden

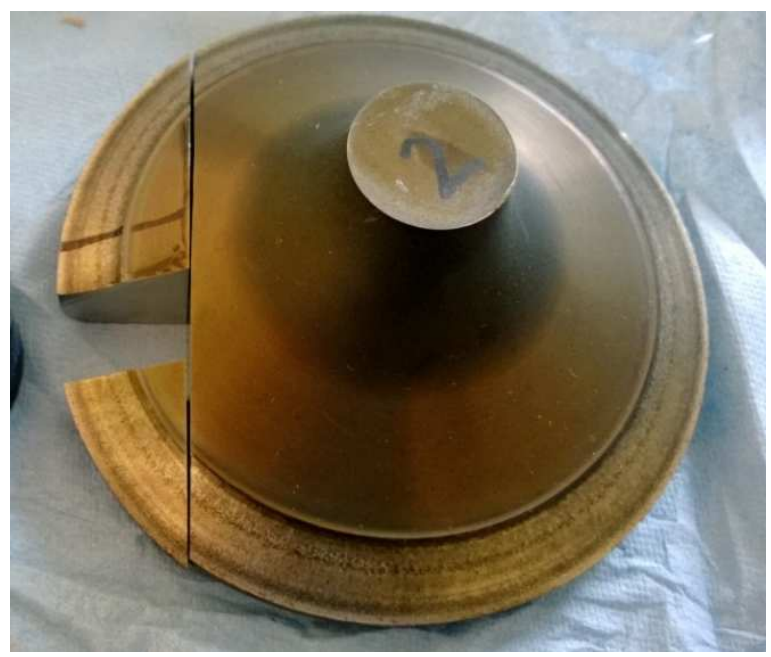

(d) A4, 250 Betriebsstunden

Abbildung 2.4: Probenübersicht, A1 bis A3 mit laufzeitabhängigen ölkohlehaltigen Ablagerungen, (a) A1 ohne erkennbare Ablagerungen, (b) A2 mit „Rußspritzern“ im Bereich der Hohlkehle, (c) A3 mit intensiven Ölkohleablagerungen in der Hohlkehle, (d) A4 mit korrodierter Oberfläche und ohne erkennbare Ölkohle 


\section{Probe A1 nach 3 Betriebsstunden}

Der Ventilteller der Probe A1 weist im Bereich der Hohlkehle keine erkennbaren Ablagerungen auf (Abbildung 2.4 (a)). Vielmehr erscheint die gesamte Oberfläche metallisch glänzend wie bei einem Neuteil. In der Detailansicht stellt sich die Kontaktfläche als linienförmige Verschleißspur im Bereich des SADs mit einer Verschleißmarkenbreite von ca. $1 \mathrm{~mm}$ dar (Abbildung 2.5 (a)), wobei das fertigungstypische Schleifbild nach dem Sitzfertigschleifen zu sehen ist. Der Ventilsitz wurde mit der Rauheit Ra 0,4 geschliffen. Man beobachtet in der Detailaufnahme Abbildung 2.6 (a) eine Glättung der Schleifspitzen infolge des Reibkontakts. In radialer Richtung sind makroskopische Verschleißriefen zu erkennen, deren Ursache unklar ist. Die Art der Glättung wird in Abbildung 2.6 (b) als Fältelung deutlich, wobei die gefältelten Schleifspitzen tieferliegende Schleifriefen nicht einglätten. Die Riefen können Artefakte eines Kornüberstands beim Sitzfertigschleifen sein. Die Überlagerungen an den Schleifriefen stützen die Beobachtung einer Oberflächenglättung infolge eines radialen Scherens der Schleifspitzen (Abbildung 2.6(b)). Makroskopisch erscheint die Kontaktfläche verschleißlos. Mikrostrukturelle Veränderungen lassen sich erst im geätzten Querschliff in Form von Versetzungen und Zwillingsbildung innerhalb der metallischen Zellen erkennen und bestätigen somit die Beobachtungen einer radialen Scherbeanspruchung aus der Draufsicht (Abbildung 2.9 (a)).

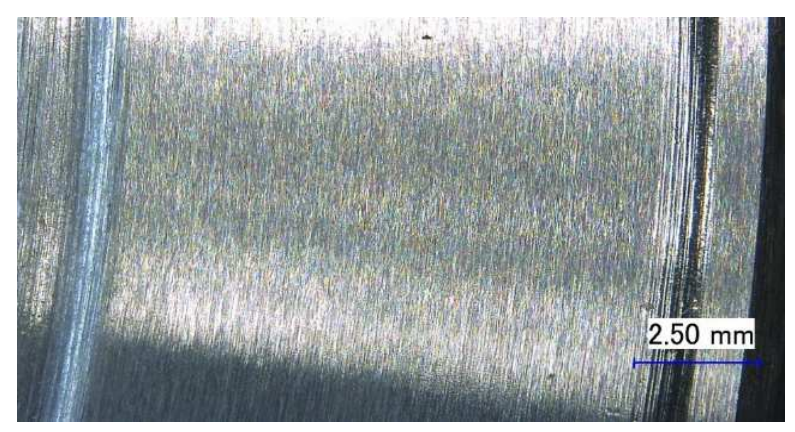

(a)

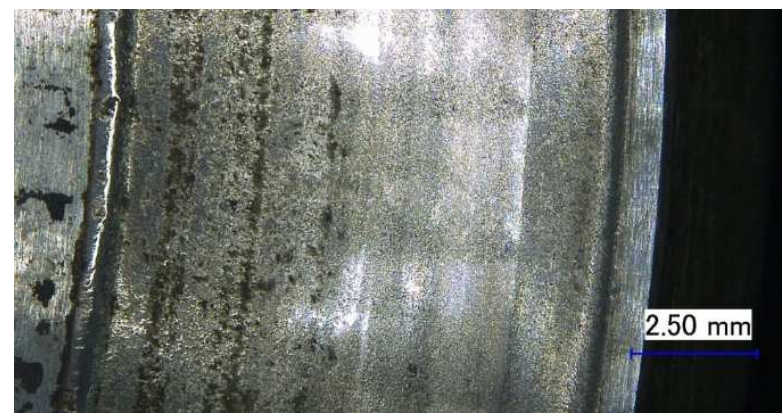

(c)

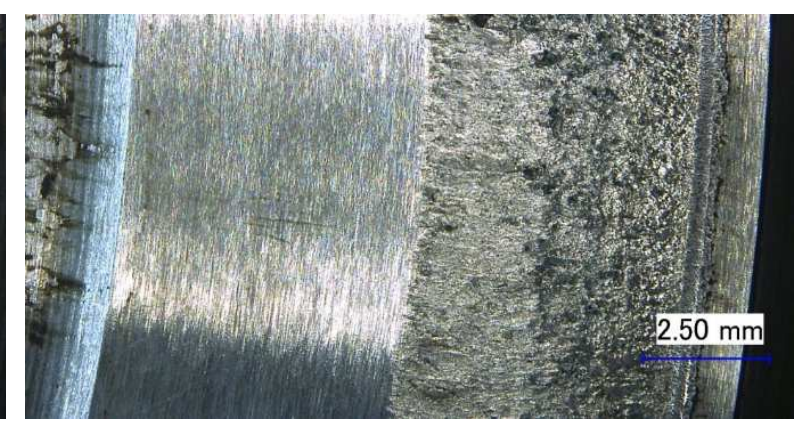

(b)

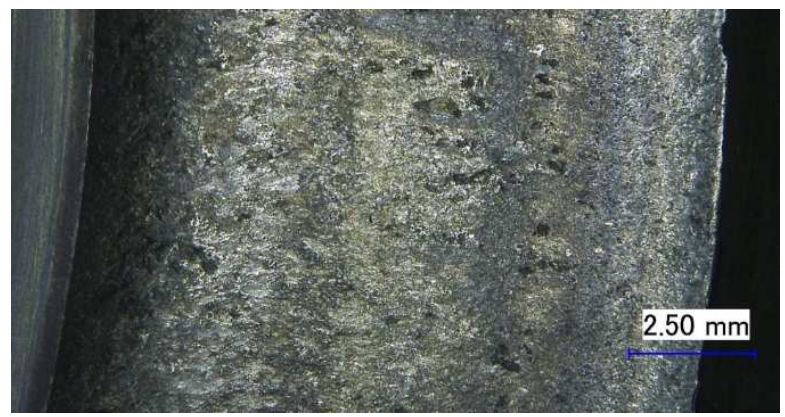

(d)

Abbildung 2.5: Übersicht der Ventilsitzflächen, (a) Probe A1 mit linienförmiger Verschleißspur (Linientrag) im Bereich des SADs, (b) Probe A2 mit aufgerauter Kontaktfläche und Verschleißmarkenbreite von ca. $6 \mathrm{~mm}$, (c) Probe A3 mit geglätteter Verschleißspur und Verschleißmarkenbreite über die gesamte nominelle Ventilsitzbreite, (d) Probe A4 mit aufgerauter Kontaktfläche und größerer Verschleißmarkenbreite als nomineller Ventilsitzbreite 
Neben Scherbändern sind in der Detailaufnahme Abbildung 2.10 (a) vereinzelte Risse in den dendritischen Karbidstrukturen der untereutektischen Stellite ${ }^{\mathrm{TM}}$ 12-Panzerung zu erkennen. Diese können als Folge der akkumulierten Versetzungen an den Karbiden angesehen werden, da die Gleitebenen der metallkeramischen Hartphasen, in der Hauptsache $\mathrm{Cr}$ - und W-Karbide, blockiert sind. Eine verschleißminimierende Triboschicht wurde nicht beobachtet.

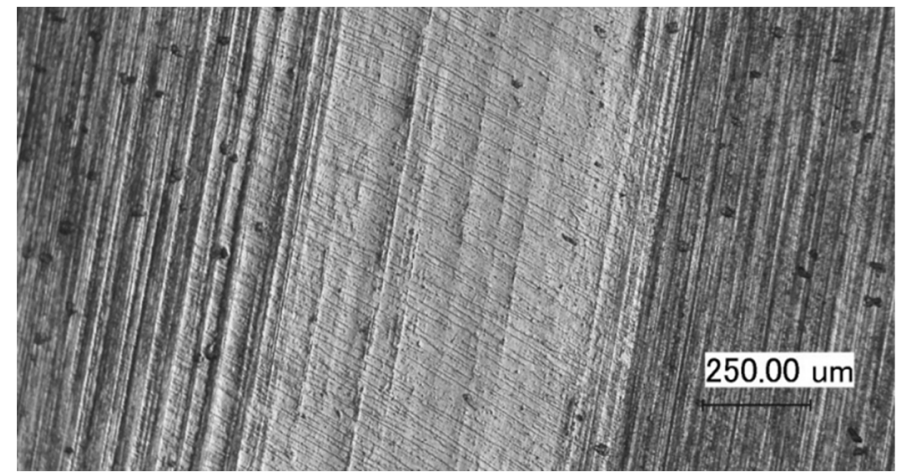

(a)

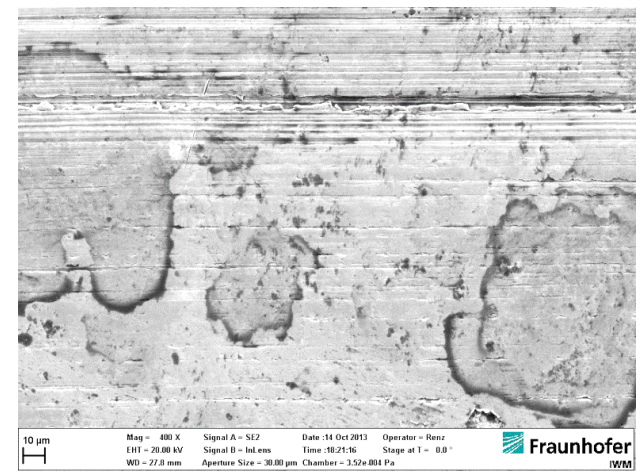

(b)

Abbildung 2.6: (a) REM-Detailaufnahme aus Abbildung 2.5 (a) mit einer Glättung der Schleifspitzen infolge des Reibkontakts, (b) Fältelung der Schleifspitzen in radialer Richtung zum SAD mit erkennbaren Schleifriefen; Vergrößerung $400 \mathrm{fach}$

\section{Probe A2 nach 30 Betriebsstunden}

Am Ventilteller der Probe A2 sind vom Übergang Hohlkehle/Hals bis zum Ventilsitz spritzerartige Verbrennungsrückstände, vermutlich aus verbranntem Öl und Kraftstoff, als Ablagerungen zu beobachten (Abbildung 2.4 (b)). In der lichtmikroskopischen Draufsicht stellt sich die Kontaktfläche mit einer radialen Verschleißmarkenbreite von ca. $6 \mathrm{~mm}$ deutlich breiter im Vergleich zu A1 dar und entspricht damit in etwa der halben nominellen Ventilsitzbreite (Abbildung 2.5 (b)). Daneben sind oberflächlich Mulden und Kuppen erkennbar. Die REM-Aufnahmen charakterisieren die Verschleißspur mit einer eingeglätteten Oberfläche, flachen Ausbrüchen, Grübchen im Bereich des SIDs (Abbildung 2.7 (a)) sowie Kuppen, Schuppen und Löchern im Bereich der SM (Abbildung 2.7 (b)). Es sei angemerkt, dass die Verschleißerscheinungen in den Bereichen SID und SAD vergleichbar sind. Im Querschliff der geätzten Probe wird nach 30 Betriebsstunden neben einer topographisch hervorstehenden Dendritenstruktur ein Dehnungsgradient bis in eine Tiefe von ca. $60 \mu \mathrm{m}$ deutlich. Unterhalb der Oberfläche ist eine mechanisch gemischte Randschicht mit einer Dicke von bis zu $20 \mu \mathrm{m}$ zu beobachten (Abbildung 2.9 (b)). Charakteristisch für die gemischte Schicht ist eine korngefeinte Mikrostruktur aus feinverteilten Karbidbruchstücken eingebettet in eine plastisch verformte Matrix. Darunterliegend ist im Dehnungsgradienten eine gebrochene und radial gerichtete Hartphasenstruktur zu erkennen. In Analogie zur Probe A1 ist keine verschleißminimierende Triboschicht zu finden. 


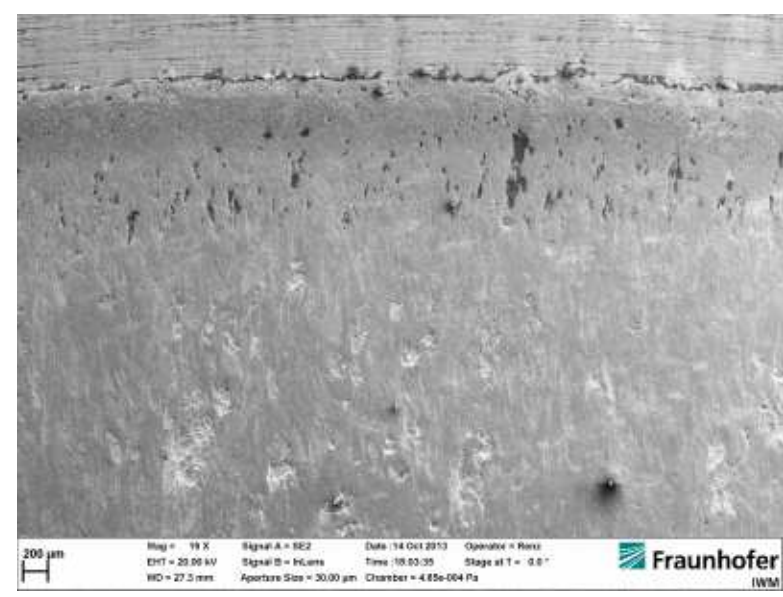

(a)

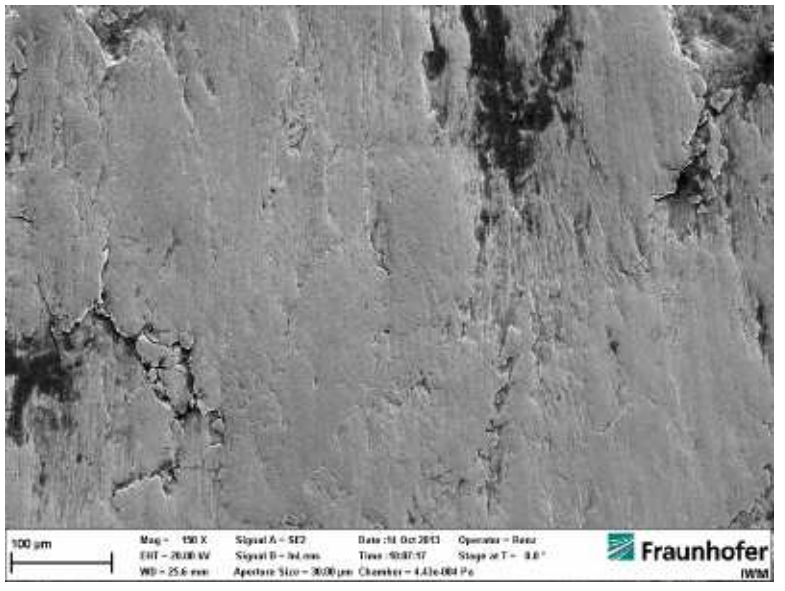

(b)

Abbildung 2.7: REM-Draufsichten der Verschleißspur von Probe A2, (a) SID, (b) SM

\section{Probe A3 nach 250 Betriebsstunden}

Die Probe A3 weist mit der längsten Laufzeit im Vergleich zu A1 und A2 den größten Ablagerungsgrad an Verbrennungsrückständen im Bereich der Hohlkehle auf (Abbildung 2.4 (c)). Die Verschleißmarkenbreite erstreckt sich über den nominellen Ventilsitz hinaus, wobei die Verschleißspur makroskopisch im Kontrast zu A1 und A2 glatt und glänzend erscheint (Abbildung 2.5 (c)). Visuell hat man den Eindruck, daß ein größerer Anteil an Verbrennungsrückständen im Bereich des SIDs (dunkle Bereiche) im Vergleich zum SAD zu erkennen ist. Die REM-Aufnahmen in der Draufsicht aus dem Bereich der SM stützen den makroskopischen Eindruck von einer geglätteten Oberfläche (Abbildung 2.8 (a)), wobei die Verschleißspur mit flachen Löchern, Ausbrüchen, erkennbaren Schuppen und eingedrückten Verbrennungsrückständen charakterisiert werden kann.

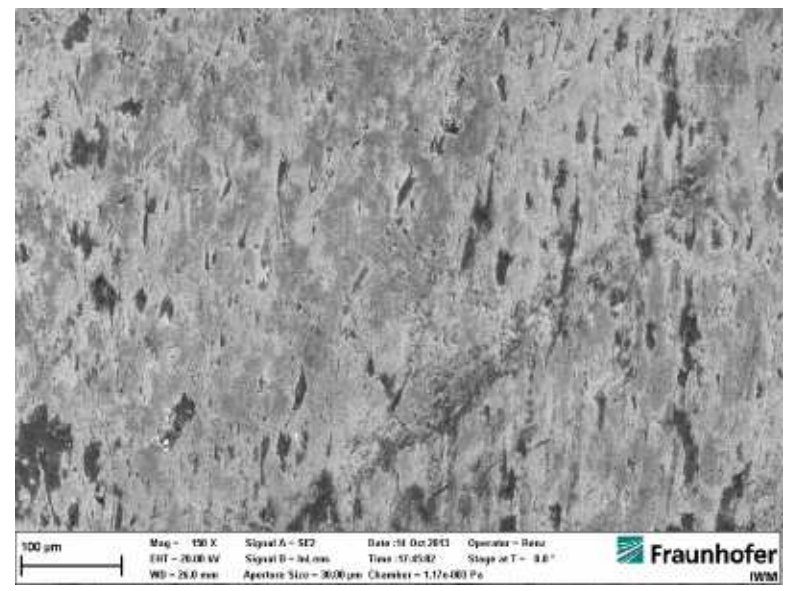

(a)

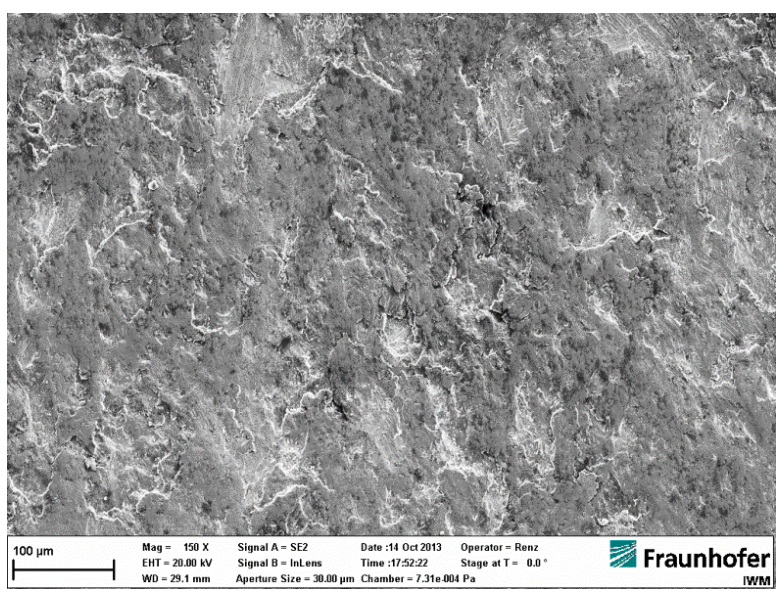

(b)

Abbildung 2.8: REM-Aufnahmen der Draufsicht aus dem Bereich der SM, (a) Probe A3 mit geglätteter Verschleißfläche und eingedrückten Verbrennungsrückständen sowie Löchern, (b) Probe A4 mit deutlich topografierter Oberfläche und Kuppen, Schuppen sowie Löchern 
Abbildung 2.9 (c) veranschaulicht im geätzten Querschliff eine ca. $20 \mu \mathrm{m}$ tiefgehende plastische Scherzone, wobei sich die auftraggeschweißte Hartphasenstruktur in Bezug auf die Dendritenausbildung $z u$ den Proben $A 1$ und $A 2$ unterscheidet. In der Detailaufnahme Abbildung 2.10 (c) ist im Vergleich zur Probe A1 ein Dehnungsgradient mit gebrochenen Hartphasen zu erkennen. Der Gradient weist eine Tiefenausdehnung von ca. $8 \mu \mathrm{m}$ auf und ist radial zum SAD gerichtet. Analog zur Probe A1 sind innerhalb der metallischen Zellen nach Ätzung Scherbänder in Form von Versetzungen zu erkennen. Als Gemeinsamkeit mit den Proben A1 und A2 konnte auf der Oberfläche von A3 keine Triboschicht nachgewiesen werden.

\section{Probe A4 nach 250 Betriebsstunden}

Im Kontrast zu den Proben A1 bis A3 finden sich an A4 im Bereich der Hohlkehle nahezu keine ölkohlehaltigen Ablagerungen (Abbildung 2.4 (d)). Vielmehr erscheint die Oberfläche angelaufen und korrodiert.

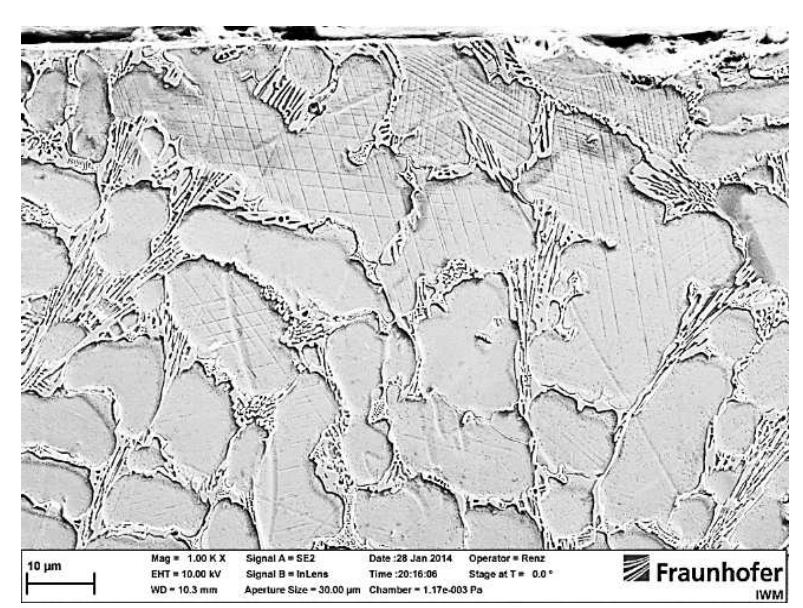

(a), geätzt

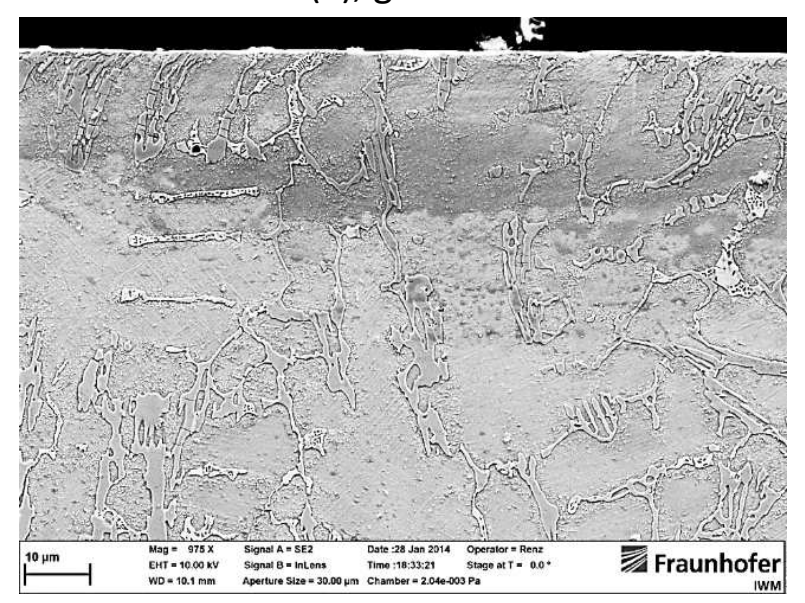

(c), geätzt

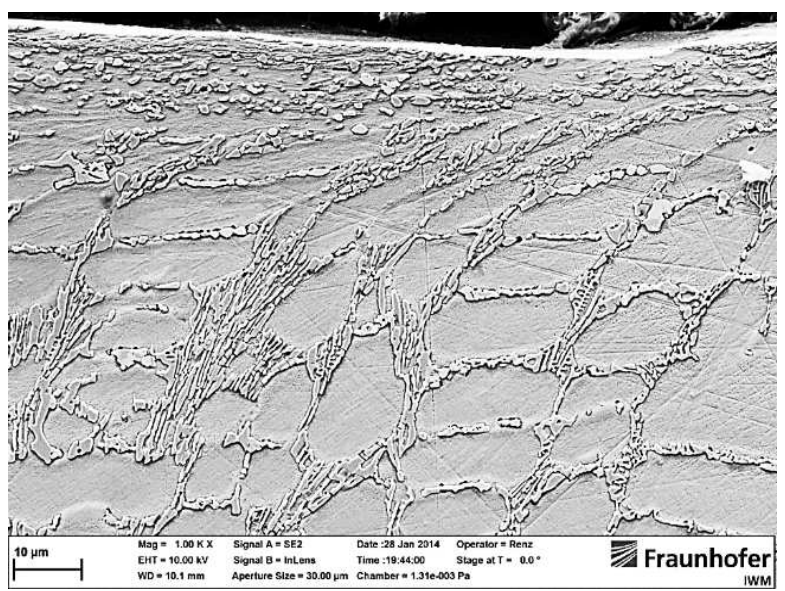

(b), geätzt

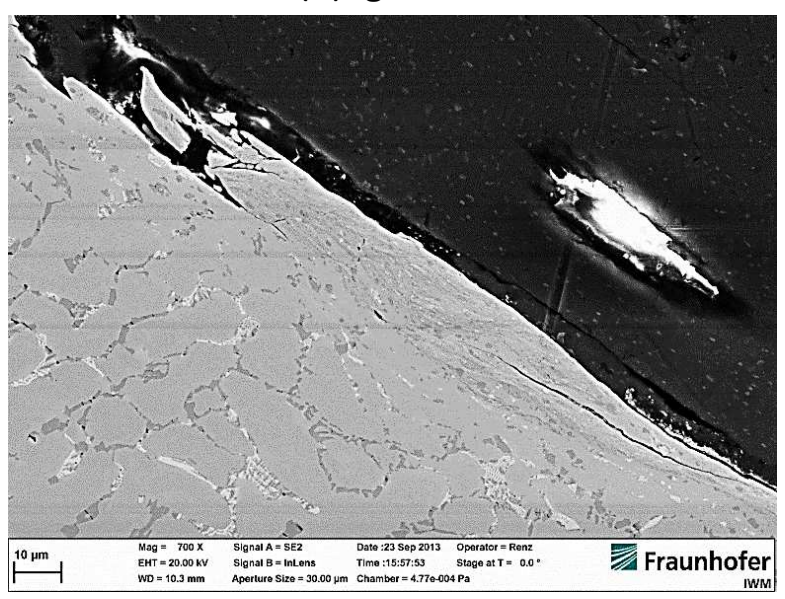

(d), ungeätzt

Abbildung 2.9: REM-Aufnahmen vom Querschliff, (a) A1 mit Scherbändern, (b) A2 mit Dehnungsgradienten bis $60 \mu \mathrm{m}$ Tiefe und mechanisch gemischter Randschicht, (c) A3 mit Dehnungsgradienten, (d) A4 mit parallelen Rissen zur Oberfläche und schuppenförmigem Verschleißpartikel (Delamination) 
Makroskopisch erscheint die Verschleißspur über die gesamte Kontaktbreite deutlich aufgeraut. In der lichtmikroskopischen Draufsicht ist die im Vergleich zur nominellen Ventilsitzbreite vergrößerte Verschleißmarkenbreite auffällig (Abbildung 2.5 (d)). Daneben bestätigt sich die makroskopisch aufgeraute Erscheinung der Verschleißspur in Form von Kuppen und Löchern. In der oberflächlichen REM-Aufnahme präsentiert sich Verschleißfläche mit deutlicher Topographie in Form von Schuppen, flachen Ausbrüchen und Löchern (Abbildung 2.8 (b)). Im Querschliff beobachtet man neben kleineren einen großen schuppenförmiger Verschleißpartikel, flache Ausbrüche und Anrisse (Abbildung 2.9 (d)). Das schuppenartige Partikel weist eine Länge von mehreren Zehntelmikrometern auf, wobei die Mikrostruktur als plastisch verformt, korngefeint und mechanisch gemischt mit feinverteilten Hartphasenbruchstücken charakterisiert werden kann. Die Anrisse starten in der mechanisch gemischten Zone.

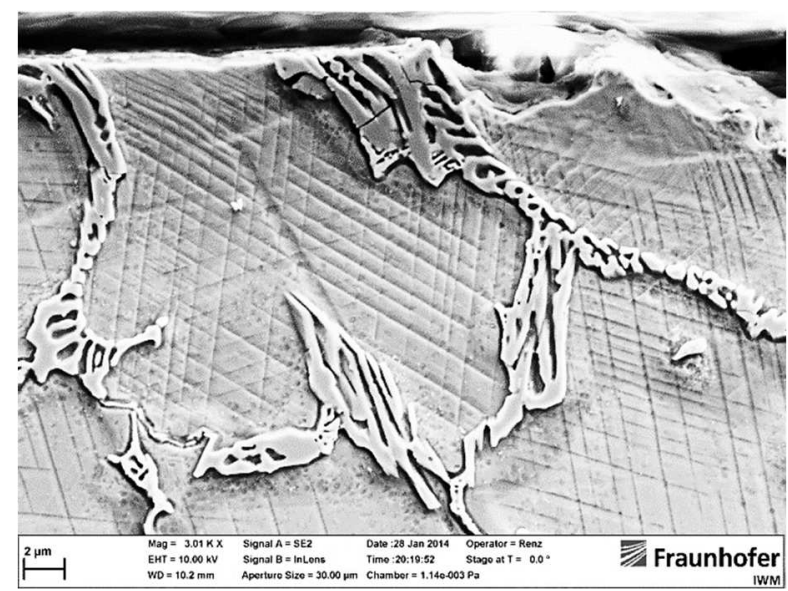

(a)

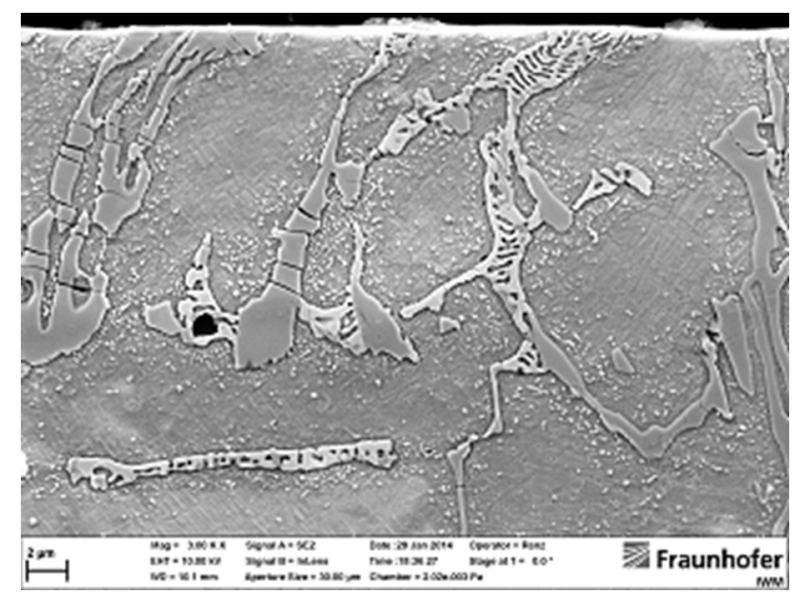

(c)

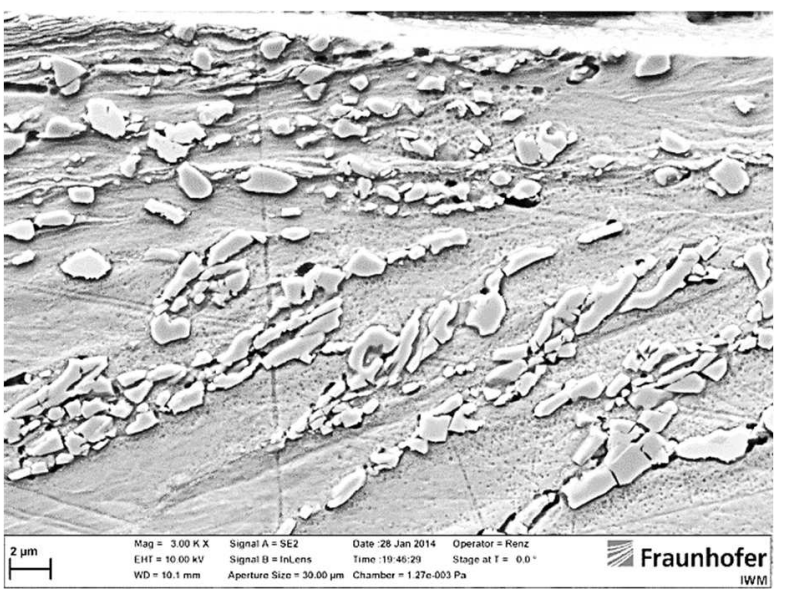

(b)

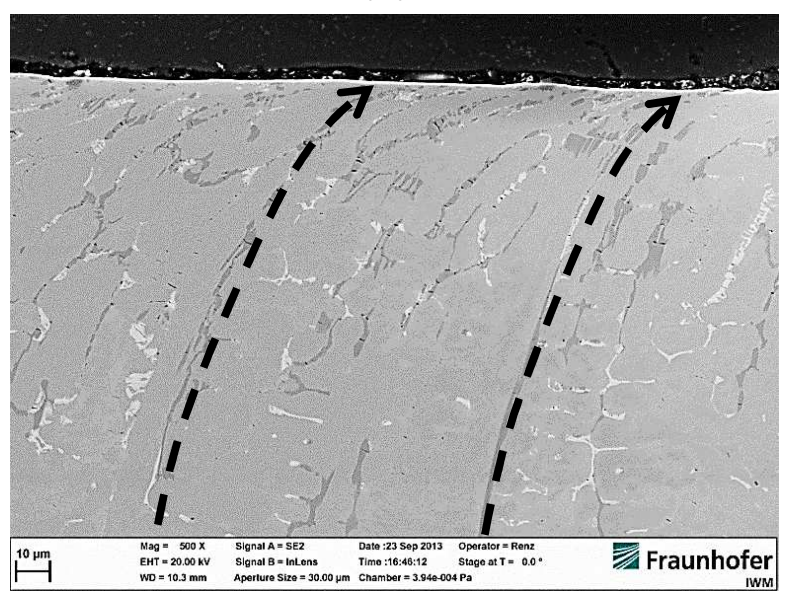

(d)

Abbildung 2.10: REM-Detailaufnahmen, (a) aus Abbildung 2.9 (a) mit Versetzungen und Zwillingsbildung in den metallischen Zellen, (b) aus Abbildung 2.9 (b) mit korngefeinter und metallisch gemischter Randschicht, (c) aus Abbildung 2.9 (c) mit zerbrochenen Karbiden im Dehnungsgradienten, (d) Probe A4 mit Dehnungsgradienten 
Kennzeichnend für Anrisse und Rissausbreitung sind ein paralleler Verlauf zur Oberfläche. Ein Dehnungsgradient ist in diesem Fall nicht zu beobachten. An einer zweiten Position der Probe A4 findet sich hingegen ein Dehnungsgradient mit radial zum SAD gerichteten Scherstrukturen und zerbrochenen Hartphasen wieder (Abbildung 2.10 (d)).

\subsubsection{Profilometrie}

In Abbildung 2.11 sind die Verschleißprofile der Proben A1 bis A4 dargestellt. Die Kontaktsituation ist im Neuzustand mit Kontakt am SAD definiert, wobei die originalen Teilkreise der SID bei allen Proben gleich sind. Außer bei der verschleißbedingten Probe A4 finden sich die originalen SID in einer radialen Distanz von ca. 1,5 $\mathrm{mm}$ in den Profilen wieder. Hingegen ist der originale SAD nur bei der Probe A1 in einer Distanz von 13,7 mm erhalten geblieben. Man beobachtet bei den Proben A1 und A2 eine kleinere Verschleißmarkenbreite im Vergleich zur nominellen Ventilsitzbreite. Bei A3 sind beide gleich groß, wohingegen bei A4 eine größere Verschleißmarkenbreite als die nominelle Sitzbreite erreicht wird. In radialer Distanz von 3 und $13 \mathrm{~mm}$ sind Peaks im Profilverlauf von A3 zu sehen, die Beginn und Ende der Verschleißmarkenbreite markieren. Mit steigender Betriebsstundenanzahl scheinen Profiltiefe und Verschleißmarkenbreite anzusteigen. Der Profilverlauf verändert sich über die Betriebsdauer. So verändert sich die Kontur anfangs für $A 1$ und $A 2$ von parallel zu konvex, um dann mit größerem Verschleiß für $A 2$ und $A 3$ von konvex zu konkav zu wechseln. Aufgrund der qualitativen Ähnlichkeit der Tiefenprofile von A3 und A4 darf für die AVS A4 ein ähnliches Verschleißverhalten angenommen werden.

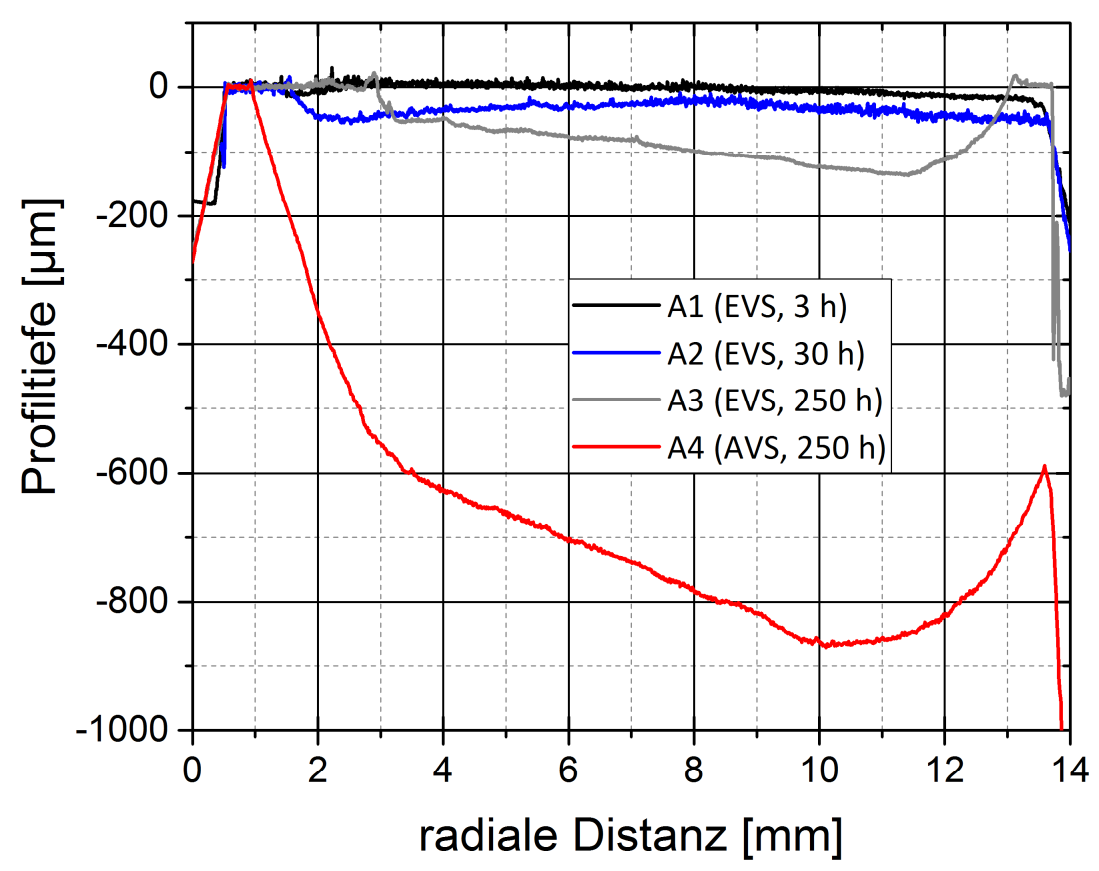

Abbildung 2.11: Profilometrie an den Ventilsitzflächen der Proben A1 bis A4, SID bei $1,5 \mathrm{~mm}$ und SAD bei $13,7 \mathrm{~mm}$ 
Die intensivere Konkavität von A4 ist mit einer maximalen Profiltiefe von $870 \mu \mathrm{m}$ in radialer Distanz von 10,1 mm in etwa um den Faktor 6 größer im Vergleich zu A3 mit $137 \mu \mathrm{m}$ an der Stelle $11,4 \mathrm{~mm}$. Für beide Proben wurden die größten Profiltiefen im Bereich des SADs gemessen. Am Tellerdurchmesser wurden deutliche Profilspitzen für A3 und A4 mit $18 \mu \mathrm{m}$ bei $13,1 \mathrm{~mm}$ bzw. - 588,7 $\mu \mathrm{m}$ bei 13,6 mm gemessen. Vergleicht man die Profile im Bereich des SIDs, scheint sich mit steigendem Verschleiß ein steilfallender Verlauf einzustellen. Bei Betrachtung aller Tiefenprofile scheint der Verschleiß am SAD zu starten.

Die ermittelten Werte für Verschleißfläche und Verschleißrate sind in Abbildung 2.12 dargestellt. Ein direkter Vergleich der beiden Kenngrößen über alle Proben ist aufgrund unterschiedlicher Belastungskollektive nicht zulässig. Es sei daran erinnert, dass die Proben A1 bis A3 EVSn und A4 AVS sind. Betrachtet man die Verschleißflächen von A1 bis A3, scheinen diese über die Zeit nichtlinear anzusteigen (Abbildung 2.12 (a)). Diese Annahme wird durch die unterschiedlich großen Verschleißraten gestützt (Abbildung 2.12 (b)). So steigt der Verschleiß von $A 1$ auf $A 2$ von 0 auf $3,95 \mathrm{~mm}^{3} / \mathrm{h}$ und reduziert sich um den Faktor 2,7 für A3 auf $1,45 \mathrm{~mm}^{3} / \mathrm{h}$. Für A4 wurde mit $11,81 \mathrm{~mm}^{3} / \mathrm{h}$ die größte Verschleißrate ermittelt. Betrachtet man die Proben A3 und A4 mit gleicher Betriebszeit, so sind Verschleißfläche und Verschleißrate von A4 in etwa um den Faktor 8 größer. Die Ergebnisse lassen einen Einfluss von Belastungsdauer und Temperatur auf das Verschleißverhalten des Tribosystems VS/SR erkennen. Darüber hinaus darf aus der Verschleißentwicklung ein nichtlineares Verschleißverhalten vermutet werden.

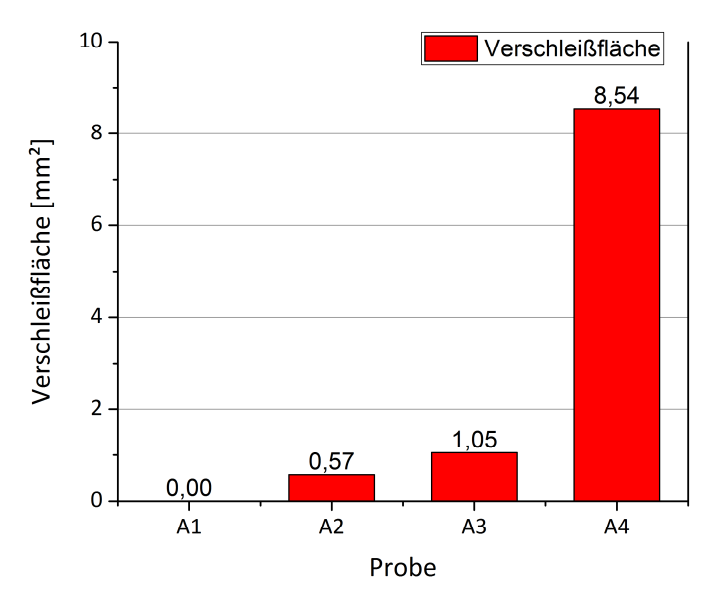

(a)

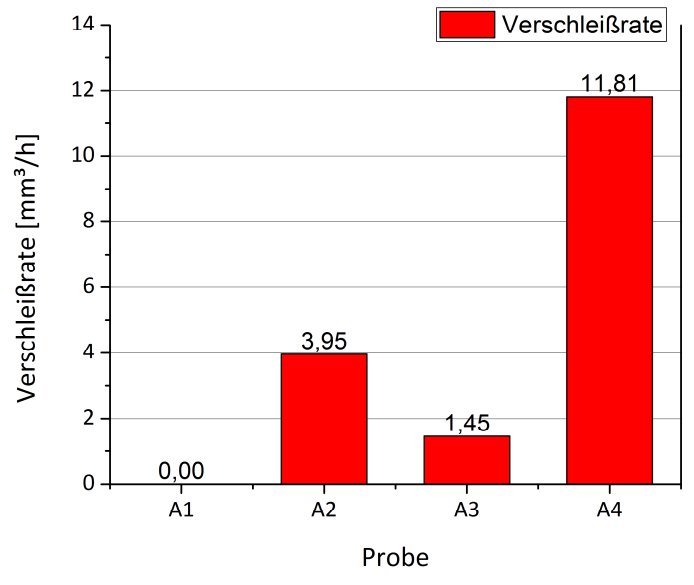

(b)

\section{Abbildung 2.12: Verschleißkenngrößen der Proben A1 bis A4, (a) Verschleißflächen in $\mathrm{mm}^{2}$, (b) Verschleißraten in $\mathrm{mm}^{3} / \mathrm{h}$}




\subsubsection{Röntgenphotoelektronenspektroskopie}

Abbildung 2.13 veranschaulicht an der Position 1 eine Referenzmessung und an der Position 2 eine repräsentative Messung in der Verschleißspur. Position 1 diente zur Bestimmung der chemischen Zusammensetzung der Stellite ${ }^{\mathrm{TM}}$ 12-Panzerung. An der Referenzposition beobachtet man für $\mathrm{O}$ bis in $20 \mathrm{~nm}$ Tiefe eine steilfallende Konzentrationskurve auf 3,5 at.-\%, die im weiteren Verlauf in eine flachfallende Kurve wechselt (Abbildung 2.13 (a)). Das gegenläufige Verhalten von $\mathrm{O}$ zu Cr korreliert mit der gemessenen Chromoxidkonzentration. $\mathrm{Cr}$ liegt oberflächlich als Oxid vor, hingegen wird Co nur als metallisch gebunden gemessen. Ab einer Tiefe von ca. $20 \mathrm{~nm}$ wird die typisch chemische Zusammensetzung von Stellite ${ }^{\mathrm{TM}} 12$ erreicht. Im Vergleich zur Referenzmessung wurde an der Position 2 in der Verschleißspur am SAD eine ähnlich hohe Sauerstoffkonzentration von ca. 3,5 at.- \% bis in eine Tiefe von $170 \mathrm{~nm}$ gemessen (Abbildung 2.13 (b)). Der Nachweis von Chromoxid bis in $60 \mathrm{~nm}$ Tiefe ist im Vergleich zur Messposition 1 um den Faktor 3 größer und weist auf eine höhere Oxidationsstufe hin. Daneben wurden die Oxide der Elemente Ca und Zn in der Verschleißspur nachgewiesen.
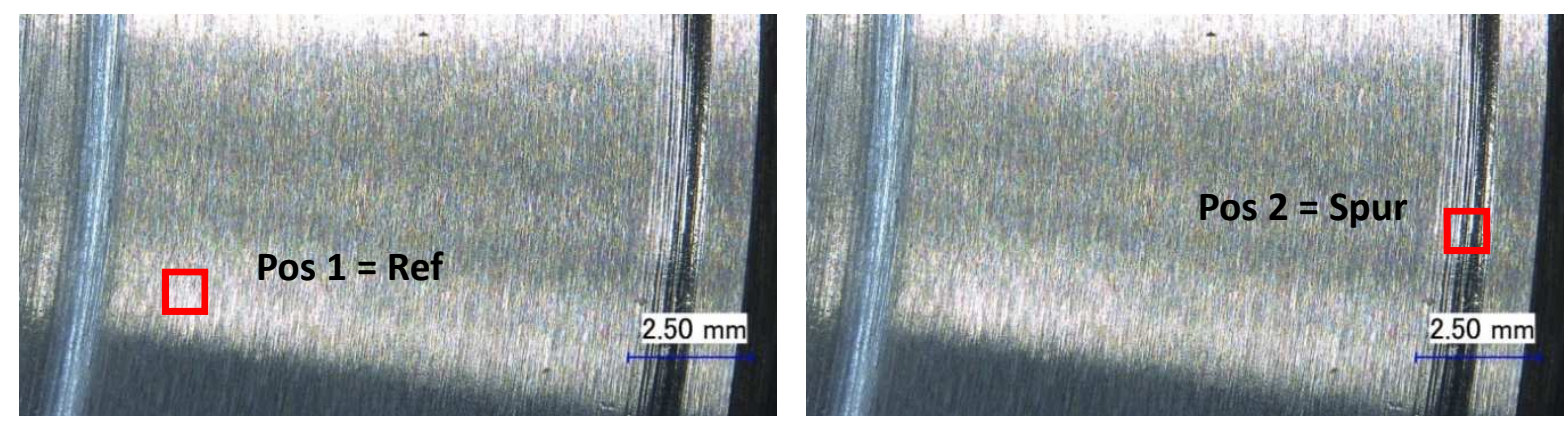

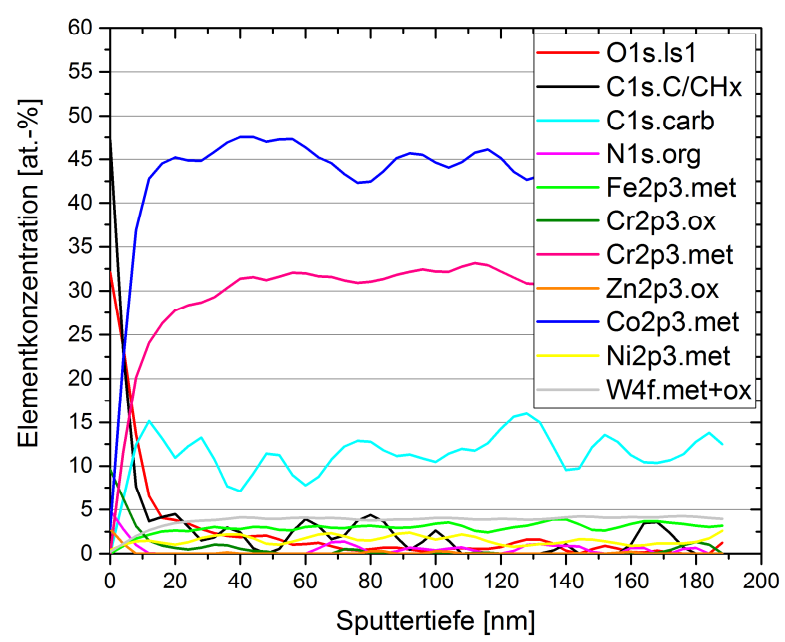

(a)

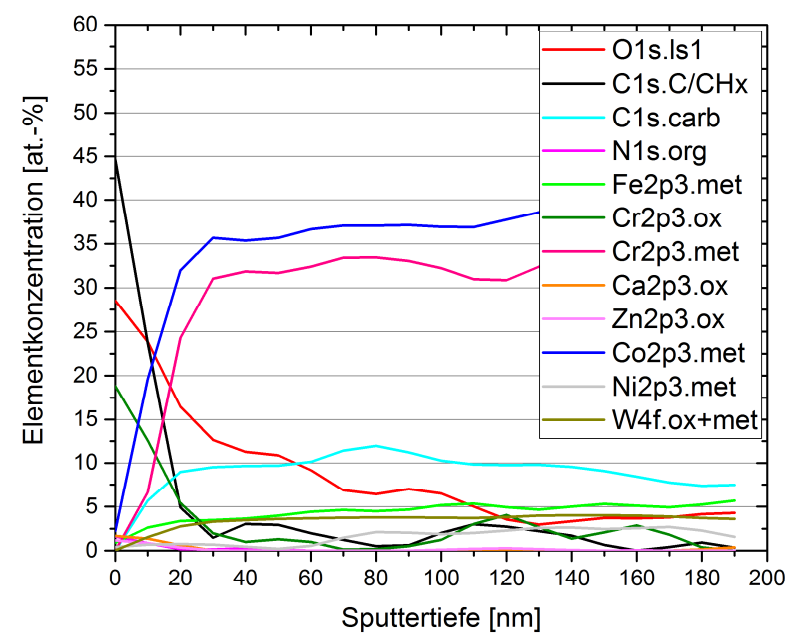

(b)

Abbildung 2.13: Atomkonzentrationen in \% als Funktion der Sputtertiefe in nm; XPSMessungen an der Probe A1, (a) Messposition 1 neben der Verschleißspur, (b) Messposition 2 in der Verschleißspur am SAD; Skalierung $60 \%$ 
Eine geänderte Skalierung der Tiefenachse von Nano- in Mikrometer verdeutlicht für die Proben A2 und A3 eine andere Größenordnung der Oxidation im Vergleich zu A1 (Abbildung 2.14). Für $A 2$ wurde bis in $0,2 \mu \mathrm{m}$ Tiefe mit 13,5 at.- $\%$ eine steilfallende Sauerstoffkonzentrationskurve gemessen, die erst am Messende in $2 \mu \mathrm{m}$ Tiefe in eine flachfallende Kurve mit 7,1 at.-\% wechselt (Abbildung 2.14 (a)). Das Sauerstoffprofil indiziert eine steigende Oxidation von Stellite ${ }^{\mathrm{TM}} 12$ mit längerer Betriebszeit. Neben $\mathrm{O}$ ist ein großer Gradient für die $\mathrm{C} / \mathrm{CHx}$-Verbindungen zu beobachten. Dieser fällt oberflächlich von 67 auf 20 at.- \% innerhalb der ersten $120 \mathrm{~nm}$ und ist bis in eine Tiefe von 1,84 $\mu \mathrm{m}$ nachweisbar, wobei in einigen Sputtertiefen die Nachweisgrenze erreicht wurde. Daneben ist ein schwankender Gehalt an karbidisch gebundenem Kohlenstoff über die gesamte Messung festzustellen.

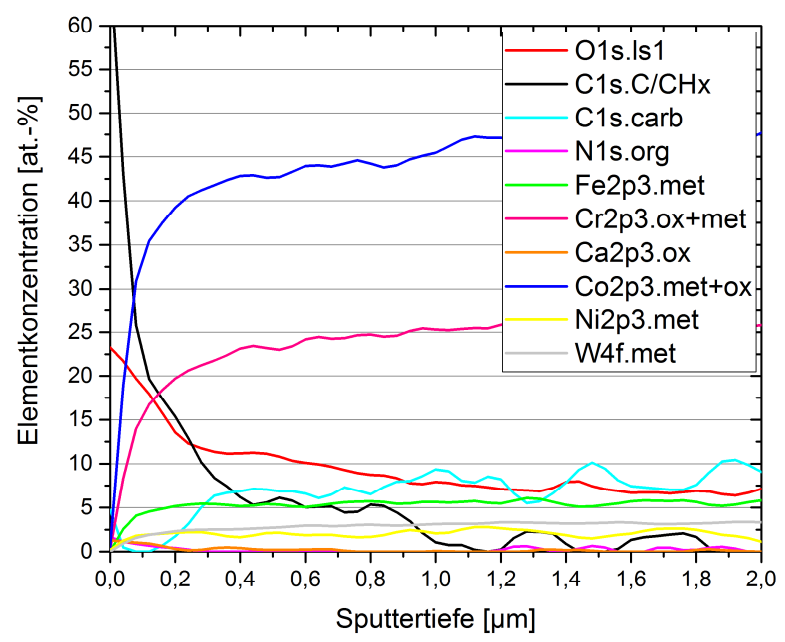

(a)

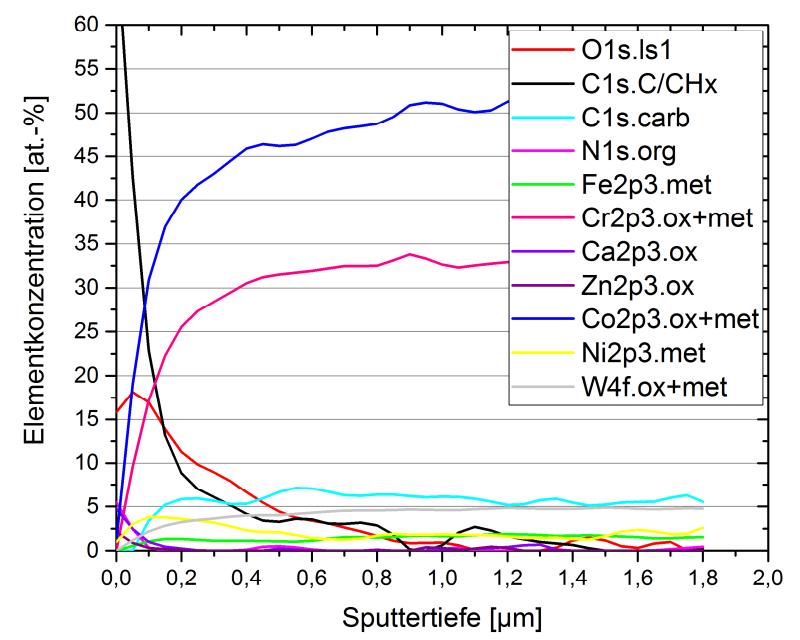

(b)

Abbildung 2.14: Atomkonzentrationen in \% als Funktion der Sputtertiefe in $\mu \mathrm{m}$; XPSMessungen im Bereich des SADs, (a) Probe A2 und (b) Probe A3; Skalierung 60 \%

An der Probe A3 kann nach 250 Betriebsstunden im Vergleich zu A2 eine höhere Oxidation an ähnlicher Position am SAD nicht nachgewiesen werden, vielmehr wurde ab 0,4 $\mu \mathrm{m}$ Tiefe ein Sauerstoffgehalt von 7 at.- \% nicht mehr überschritten (Abbildung 2.14 (b)). Als Gemeinsamkeit weisen Sauerstoff- und Chromoxidkonzentrationsprofile Unstetigkeiten auf. Eine erste Nachweisgrenze wurde für $\mathrm{O}$ in einer Sputtertiefe von 1,15 $\mu \mathrm{m}$ gemessen, wobei in 1,7 $\mu \mathrm{m}$ Tiefe wieder ein Gehalt von 1,0 at.- \% nachgewiesen werden konnte. Chromoxid war mit Nachweisgrenzen in Tiefen von 0,9 und 1,4 $\mu \mathrm{m}$ bis zum Messende in 1,8 $\mu \mathrm{m}$ Tiefe detektierbar. Hingegen verdeutlicht eine zweite Messung im Bereich des SIDs ein anderes Bild (Abbildung 2.15 (a)). Bis kurz vor Messende in $3 \mu \mathrm{m}$ Tiefe konnte $\mathrm{O}$ mit Unstetigkeiten bis in eine Tiefe von 2,94 $\mu \mathrm{m}$ nachgewiesen werden. Der Sauerstoffgehalt lag bis $0,78 \mu \mathrm{m}$ oberhalb von 7 at.- \%. Nachweisgrenzen wurden in drei Sputtertiefen bei 2,16, 2,22 und 2,64 $\mu \mathrm{m}$ gemessen. Daneben wurden Metalloxide von $\mathrm{Cr}$, Co und Ca detektiert, wobei eine getrennte Darstellung von metallisch und oxidisch gebundenem Co nicht möglich war. Abbildung 2.15 (b) veranschaulicht das Tiefenprofil von A4 mit einer auffällig hohen Sauerstoffkonzentration. 
$\mathrm{Neu}$ in diesem Untersuchungskontext ist die flachfallende Konzentrationskurve von $\mathrm{O}$ von oberflächlich 36,1 auf 17,3 at.- \% bis zum Messende in einer Tiefe von $3 \mu \mathrm{m}$ nach einem Anstieg innerhalb der ersten $100 \mathrm{~nm}$ von 13,2 auf 36,1 at.- \%.

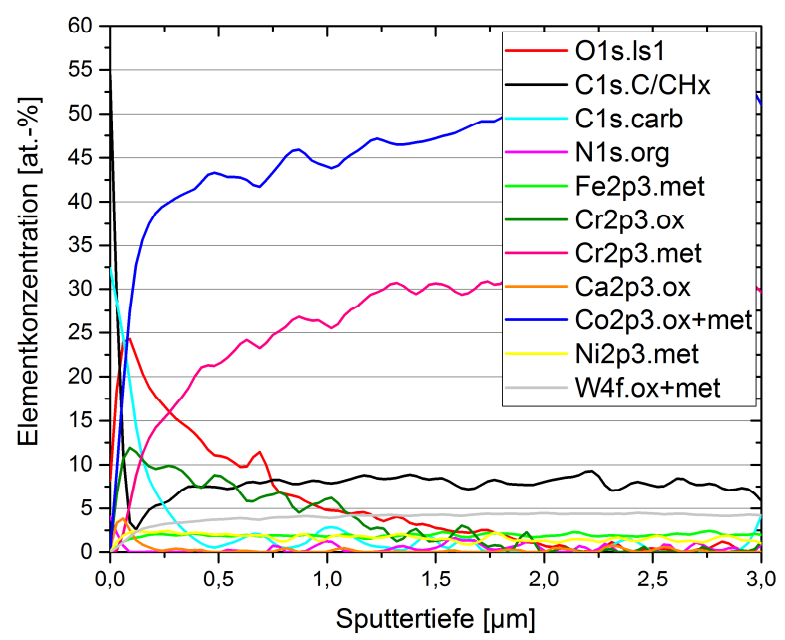

(a)

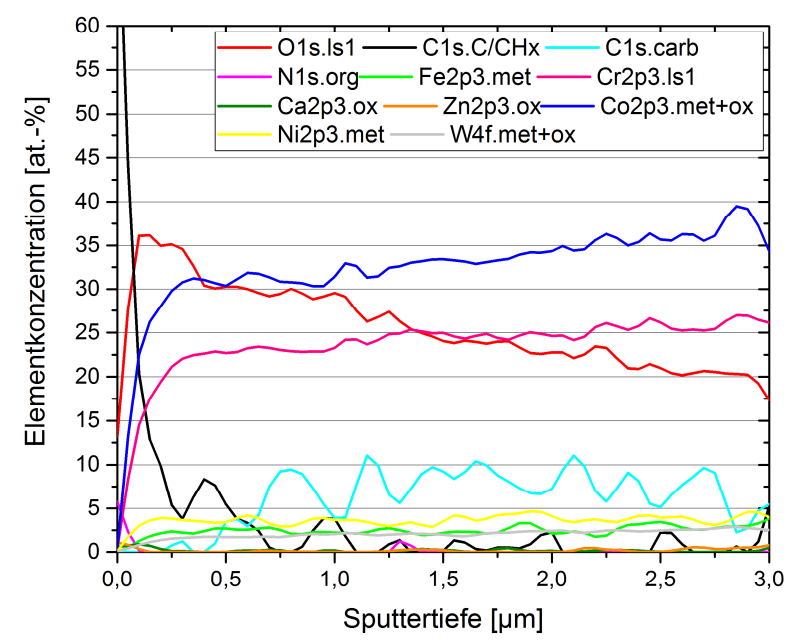

(b)

Abbildung 2.15: Atomkonzentrationen in \% als Funktion der Sputtertiefe in $\mu \mathrm{m}$; XPSMessungen, (a) Probe A3 im Bereich des SIDs, (b) Probe A4 im Bereich des SADs ; Skalierung $60 \%$

In Tabelle 2.5 ist ein Vergleich der Sauerstoffgehalte für die Proben A3 und A4 wiedergegeben. Interessant ist, der vielfach höhere Sauerstoffgehalt von A4 ab 0,05 $\mu \mathrm{m}$ Tiefe.

Tabelle 2.5: Vergleich der Sauerstoffkonzentrationen von A3 mit A4 am SAD

$\begin{array}{cccc}\text { Tiefe }[\boldsymbol{\mu m}] & \text { A3 [at.-\%] } & \text { A4 [at.-\%] } & \text { A4/A3 } \\ 0,00 & 15,8 & 13,2 & 0,84 \\ 0,25 & 9,8 & 35,1 & 3,6 \\ 0,50 & 4,4 & 30,2 & 6,9 \\ 0,75 & 2,2 & 29,5 & 13,4 \\ 1,00 & 0,9 & 29,5 & 32,8 \\ 1,25 & - & 27,4 & - \\ 1,50 & 1,2 & 24,0 & 20,0 \\ 1,80 & - & 24,0 & -\end{array}$




\subsection{Zusammenfassung und Schlussfolgerungen}

Am Beispiel von vier typgleichen VSn aus dem Feld mit unterschiedlicher Laufzeit wurden die mikrostrukturellen Veränderungen in der tribologisch beanspruchten Randschicht im Hinblick auf die eigenschaftsbestimmenden Verschleißmechanismen untersucht. Die Proben waren am Ventilsitz mit Stellite ${ }^{\mathrm{TM}} 12$ gepanzert und stammten alle aus demselben Großgasmotor. Typgleich bedeutet, dass die VSn im Neuzustand dimensions- und werkstoffgleich waren. Es wurden Verschleißraten von bis zu 3,95 und 11,81 mm³ $/ \mathrm{h}$ für EVS bzw. AVS ermittelt. Aus den gewonnenen Erkenntnissen wurden die Anforderungen an die Entwicklung eines neuen VVPs für großgasmotorische Applikationen definiert:

1) Aufbau eines Prüfstands auf Komponentenebene, um die Systemstruktur des tribotechnischen Systems weitestgehend beizubehalten, da Ergebnisse aus Modellversuchen eine eingeschränkte Übertragbarkeit zeigen [143]. Ferner verursachen Motor- oder Betriebsversuche in der tribologischen Prüftechnik einen hohen Kostenaufwand. Es sei angemerkt, dass die Anwendung einer tribologischen Prüfkette interessant jedoch zum Zeitpunkt des Projektstarts nicht aufwandskonform erschien.

2) Getrennte Untersuchung des Einflusses der Ventilphasen Schließen und Verbrennungsdruck auf das Verschleißverhalten des Tribosystems VS/SR, da nach Quellenlage diese Parameter als die beiden Hauptfaktoren betrachtet werden [43]. Darüber hinaus sind die Zuordnungen der Verschleißanteile am Gesamtventilverschleiß und die eigenschaftsbestimmenden Verschleißmechanismen für Schließen und Verbrennungsdruck nicht vollständig verstanden, vgl. [137], [141] vs. [146], [138].

3) Anwendung einer Hochtemperaturheizung zur Untersuchung des Einflusses der Temperatur auf die beiden Hauptparameter Ventilschließen und Verbrennungsdruck aufgrund fehlender Literatur für Großgasmotoren,

4) Kontrollierte Versuchsdurchführung und Parametervariation zur Beurteilung der Signifikanz einzelner Parameter auf das Verschleißverhalten,

5) Versuche mit anwendungsnahen Prüffrequenzen und Belastungen, da die bisherigen Arbeiten keinen klaren Trend erkennen lassen vgl. [142] und [147] vs. [139],

6) Einsatz einer Klimakammer zur Untersuchung des Einflusses von unterschiedlichen Atmosphären insbesondere im Hinblick auf Oxidation der tribotechnischen Werkstoffe sowie zur Bildung verschleißminimierender Triboschichten,

7) Konstruktion eines biegesteifen Prüfrahmens zur Vermeidung elastizitätsbedingter Messeinflüsse auf das Verschleißverhalten.

\section{Proben A1 bis A4 mit unterschiedlicher Laufzeit:}

Mechanisch-dominierte Mechanismen bewirken bei den Proben A1 bis A4 mit Stellite ${ }^{\mathrm{TM}} 12-$ Panzerung am Ventilsitz Verschleißraten von bis zu $11,8 \mathrm{~mm}^{3} / \mathrm{h}$. Die anfängliche Kontaktsituation verändert sich über die Zeit vom Linien- zum Flächenkontakt. Im Kontakt 
bewirken die auftretenden mechanischen Belastungen Scherspannungen in der tribologisch beanspruchten Randschicht. Wie Greenwood in [37] berichtet, wird das Verschleißverhalten im ungeschmierten Kontakt von der Deformation der Mikrokontakte bestimmt. Das untersuchte Tribosystem wurde konstruktiv mit einem Differenzwinkel zwischen VS und SR auf Außenkontakt ausgelegt (Abbildung 1.12 (b)). Daher verteilt sich der Kontakt am Anfang auf wenige Mikrokontakte im Bereich des SADs, woraus lokal hohe Flächenpressungen resultieren. Die Kontaktsituation führt zu plastischen Dehnungen, die sich an der Oberfläche durch eine Zunahme der Verschleißmarkenbreite und im Querschliff in Form von Scherbändern in den metallischen Zellen einstellt. Mikroskopisch bewirken die auftretenden Scherspannungen oberflächlich im ersten Schritt eine Fältelung der Schleifspitzen und führen in der Mikrostruktur zu Versetzungen, wie sie bei Probe A1 zu erkennen sind. Im weiteren Verlauf bilden sich auf Basis der planaren Gleitebenen in den metallischen Zellen des Co-CrMischkristalls Scherbänder aus. Die Aufakkumulation plastischer Dehnungen führt zu einem Dehnungsgradienten, wobei die eingebetteten metallkeramischen Hartphasen, in der Hauptsache W- und Cr-Karbide, aufgrund blockierter Gleitebenen zerbrechen. Bei anhaltender Belastung kommt es in Abhängigkeit von der Kontaktsituation zur Bildung einer mikrometerdicken plastisch verformten Schicht unterhalb der Oberfläche, die anschließend mechanisch gemischt wird, vgl. Probe A2 (Abbildung 2.9 (b)). Es darf angenommen werden, dass die zerbrochenen Karbide durch zyklisch auftretende Scherprozesse im Kontakt in die plastisch verformte Randzone transportiert werden. Die plastisch deformierte Randschicht hat somit ihre Ursache in einer zyklisch adiabatischen Scherung mit einer zum SAD gerichteten Dehnungsakkumulation. Aufgrund des Differenzwinkels können entstehende Verschleißpartikel den Reibspalt nur in Richtung des SIDs verlassen. In der Anfangsphase kommt es daher zu einem Materialaufwurf im Bereich der SM, wie es das konkave Verschleißtiefenprofil von A2 verdeutlicht. Im weiteren Verlauf verlassen einige Verschleißpartikel das System und erklären den Werkstoffverlust, andere Partikel verbleiben wegen des zyklischen Kontakts zeitlich unbestimmt im Reibspalt. Die gefundenen Intendationen sowie Kuppen und Schuppen belegen erscheinungsseitig diese Annahme. Mit zunehmenden Werkstoffverlust am SAD darf eine Vergrößerung der Verschleißmarkenbreite bis zum Erreichen der nominellen Ventilsitzbreite angenommen werden, vgl. Probe 3 (Abbildung 2.5 (c)). Verschleißinduzierte Oberflächenveränderungen können zu raueren Oberflächen führen, wodurch die wahre Kontaktfläche reduziert und lokale Scherspannungen vergrößert werden können. Ferner führt eine reduzierte Kontaktfläche zu höheren Temperaturen aufgrund des reduzierten Wärmeübergangs am SR. Lokal hoher Verschleiß kann die Folge sein. Daneben lassen mechanisch-dominierte Verschleißmechanismen, wie sie hier vorliegen, vermuten, dass sich nach wiederholtem Be- und Entlasten kein Steady-State einstellt [177]. Ursache können sich verändernde Kontaktgeometrien infolge plastischer Deformationen und verbleibende Verschleißpartikel im Reibspalt sein. Der mittlere Kontaktdruck $p_{m}$ muss demnach 3,69-mal größer sein als die Fließspannung unter reiner Scherspannung $\tau_{y}$. Berücksichtigt man, dass sich nach [178] die reale Kontaktfläche unter kombinierter Last von Normal- und Tangentialkraft vergrößert, wird klar, das selbst dieser Effekt bei der herrschenden mechanisch-dominierten Kontaktsituation im Hinblick auf eine 
Verschleißminimierung wirkungslos bleibt. Die vom Tellerdurchmesser in Richtung Schaft zunehmende Biegesteifigkeit bewirkt im Kontakt Stick-Slip-Effekte. Anhaften am SAD und Gleiten in den Bereichen SM und SID sind vorstellbar. Dadurch kann erhöhter Verschleiß im Bereich des SADs erzeugt werden, wie es an den konkaven Verschleißprofilen der Proben A3 und A4 zu erkennen ist. Die Einbauposition von A3 war Einlass, die von A4 war Auslass. Der mit steigender Temperatur sinkende E-Modul führt zu einer reduzierten Biegesteifigkeit des Ventiltellers, wodurch sich die Stick-Slip-Effekte am Ventilsitz einer AVS erhöhen. Aus der XPSAnalyse konnte keine Bildung reibungsreduzierender Oxide nachgewiesen werden, die nach Quinn [179], [180], [181] und Stott [51] den Verschleiß minimieren können. Daher wird über ein zunehmendes Gleiten mehr Reibleistung induziert. Diese wird vermehrt in plastische Dehnungen umgesetzt und erklärt die Konkavität am SAD. Überlagert wird dieser mechanischdominierte Mechanismus von tribooxidativen Prozessen wie die gemessene Oxidationsstufe der Probe A4 belegt. Nach Woodford und Bricknell [182] darf angenommen werden, dass ein erhöhter Oxidationsgrad zur Reduktion der Haftfestigkeit an der Grenzfläche metallkarbidische Hartphase und Matrix führt. Zerbrochene Karbide können somit herausgelöst werden, woraus eine geänderte Verschleißbeständigkeit folgt. In Erweiterung zur EDS wurde die XPS mit dem Ansatz eingesetzt, um neben der quantitativ chemischen Zusammensetzung der tribologisch beanspruchten Randschicht das Eindringen von Sauerstoff in den tribotechnischen Werkstoff hoch aufzulösen. Die Tiefenprofilanalysen der Probe A1 stützen die Annahme, dass sich infolge des befeuerten Betriebs höhere Oxidationsstufen im Stellite $^{\mathrm{TM}} 12$ einstellen. Wenn gleich bei A2 bis A4 kritisch anzumerken ist, daß die XPSErgebnisse mit einer Informationstiefe von 0,5 bis $5 \mathrm{~nm}$ in Abhängigkeit von der Anregungsenergie [176] zu Fehlinterpretationen führen können. Da die mechanisch gemischte Randzone teilweise mehrere Mikrometer dick ist, können somit Oxide von der Oberfläche in tiefere Zonen eingetragen werden und zu mutmaßlich höheren Sauerstoffkonzentrationen führen. Die nachgewiesenen Unstetigkeiten für Sauerstoff und Chromoxid stützen diese Vermutung. Als Erkenntnis ist festzuhalten, daß eine hochauflösende Analytik für mechanisch gemischte Randschichten zur Bestimmung von Oxidationsstufen eingesetzt werden kann. Temperaturinduzierte Effekte bewirken eine reduzierte Warmfestigkeit sowie einen höheren Oxidationsgrad und können somit Verschleißraten von bis zu $11,81 \mathrm{~mm}^{3} / \mathrm{h}$ für Probe A4 erklären.

Ein von der Oberfläche ansteigender Fe-Gehalt auf ca. 5 at.- \% am Messende ist allen Proben gemein. Dieses Messergebnis erklärt sich zum Großteil aus der Aufmischung der 2-lagigen PTA-Auftragschweißung. Die Mikrostrukturanalyse lieferte keine Hinweise auf eine verschleißminimierende Triboschicht. Neben kleineren Mengen an oberflächlich detektierten Verbrennungsrückständen, die in ihrer Menge nicht ausreichend für die Bildung einer Triboschicht zu sein scheinen, wirkt die mechanisch-dominierte Kontaktsituation der Bildung einer Triboschicht entgegen. In der Folge kommt es zum Metall-Metall-Kontakt mit hohen Verschleißraten und Verschleißerscheinungsformen nach Archard und Hirst [47]. 
Die beobachteten Verschleißerscheinungen wie Mulden, Kuppen und Schuppen an den Oberflächen sowie plastischen Dehnungen mit teilweise ausgeprägten Dehnungsgradienten und plastisch verformten Randschichten unterhalb der Oberfläche sind typisch mikroskopische Merkmale der Adhäsion. Daneben wurden mit parallelen Rissen zur Oberfläche und flachen Löchern an der Oberfläche charakteristische Erscheinungen der Oberflächenzerrüttung gefunden. Als eigenschaftsbestimmend können somit die beiden mechanisch-dominierten Verschleißmechanismen Adhäsion und Oberflächenzerrüttung mit Delamination festgestellt werden.

Adhäsion und Oberflächenzerrüttung bestimmen mit unterschiedlicher Gewichtung die beiden Verschleißarten Gleit- und Stoßverschleiß. Die Werkstoffanforderungen an Gleit- und Stoßverschleiß sind unterschiedlich und können eingeschränkt in einem Werkstoff zusammengeführt werden. Daher sind die Zuordnungen der Verschleißmechanismen und Verschleißanteile zu den beiden Ventilphasen Schließen und Verbrennungsdruck insbesondere in quantitativer Hinsicht für ein besseres Verständnis des tribologischen Verhaltens von VS/SR bedeutend. Die Entwicklung, Konstruktion und der Aufbau eines neuartigen VVPs mit getrennter Untersuchung der Ventilphasen Schließen und Verbrennungsdruck hatte diese Zuordnungen zum Ziel und wird im folgenden Kapitel vorgestellt. 


\section{Entwicklung, Konstruktion und Aufbau eines neuartigen Ventilverschleißprüfstands}

Aus dem Literaturstudium und den Erkenntnissen aus Kapitel 2 gehen eine Vielzahl potenzieller Einflussgrößen auf das Tribosystem VS/SR hervor, wobei Signifikanzen bekannt jedoch im Detail nicht vollständig verstanden sind. Die Entwicklung des neuartigen VVPs fokussierte auf die beiden Hauptparameter Ventilschließen und Verbrennungsdruck. Ziel war der Aufbau eines Prüfstands auf Komponentenebene, der nicht möglichst genau die realen Betriebsbedingungen nachbildet, sondern eine Prüfanordnung schafft, die eine experimentelle Simulation der eigenschaftsbestimmenden Verschleißmechanismen und Verschleißraten für die beiden Ventilphasen Schließen und Verbrennungsdruck ermöglicht.

\subsection{Prüfstandskonzept}

Die technische Umsetzung zur Untersuchung der Einflüsse der beiden Ventilphasen Schließen und Verbrennungsdruck auf das Verschleißverhalten des Tribosystems VS/SR bedeutet zwei getrennte Prüfsysteme. Da in der wissenschaftlichen Literatur kein derartiges Prüfsystem für großmotorische Applikationen auf Komponentenebene beschrieben ist, bedeutet diese Aufgabe eine Neuentwicklung. Prüfmodus I soll das Ventilschließen und Prüfmodus II den Verbrennungsdruck simulieren. Für die Realisierung sind grundsätzliche Überlegungen zur Krafteinleitung und zum Antrieb der Prüfeinrichtung anzustellen. Zu den Prüfeinrichtungen gehören Werkstoffprüfmaschinen und Prüfanlagen. Nach DIN 51220 sind Werkstoffprüfmaschinen Maschinen und Geräte, mit denen infolge einer mechanischen Beanspruchung mechanisch-technologische Eigenschaftswerte von Proben, Formelementen, Bauteilen und -gruppen bestimmt werden. Maschinen und Geräte zur Verschleißprüfung fallen nicht unter diese Definition. Erismann beschreibt in [183] den Unterschied zwischen Werkstoffprüfmaschinen und Prüfanlagen darin, dass Prüfmaschinen Prüfsysteme mit fester räumlicher Anordnung der kraftführenden Teile und Prüfanlagen Systeme mit von Versuch zu Versuch veränderter Anordnung der kraftführenden Teile sind. Definitionsgemäß ist der zu entwicklende VVP somit eine Prüfmaschine. Nach [183] bestehen Prüfmaschinen aus Krafteinleitung, Antrieb, Steuerung, Reaktionsstruktur, Energieversorgung, Messgeräten, Datenausgabe und einer Programmierung. Temporäre Teilsysteme werden nach [184] als Zusatzgeräte bezeichnet. Der Krafteinleitung kommt eine besondere Rolle zu insbesondere dem Teilsystem, das direkt auf den Prüfling wirkt. Jeder Fehler bei der Konstruktion oder Anwendung kann das Messergebnis potenziell beeinflussen und damit zu falschen Schlussfolgerungen führen.

Die Krafteinleitung im Prüfmodus I soll, in Analogie zur realen Anwendung, über das Ventilschaftende erfolgen. Dies erscheint im Hinblick auf die Systemstruktur des Prüfsystems relevant. Hingegen soll die experimentelle Simulation von Prüfmodus II nicht mittels Gasdruck, wie im realen Motorbetrieb, sondern über eine druckäquivalente Stempelkraft nachgebildet werden. Gründe hierfür sind einerseits die höheren Kosten für den mechanischen Aufbau 
einer Kolbenmaschine zur Gasdruckerzeugung durch die Baugruppe Kolben, Pleuel, Kurbelwelle und Zylinder sowie andererseits die vorhandene Erfahrung bei der Aktuatorik von Stempelzylindern. Darüber hinaus wird kein erkenntnisrelevanter Mehrwert bei Anwendung von Gasdruck erwartet.

\subsubsection{Maschinengestell und Aktuatorik}

Auf dem Gebiet der Prüfstandstechnik unterscheidet Kutter 2013 in [185] zwei Arten von Maschinengestellen, die Universal- oder Sonderlösung. Sonderlösungen sind im Aufbau effektiv, jedoch unflexibel in Bezug auf andere Prüfanwendungen und eignen sich gut für gleichartige Aufgaben bei einer wirtschaftlich hohen Auslastung. Für diese Arbeit standen zwei Prüfgestelle zur engeren Auswahl. In Abbildung 3.1 sind die beiden Varianten einer Universalund Sonderlösung mit möglichen Versuchsaufbauten als CAD-Modell dargestellt.

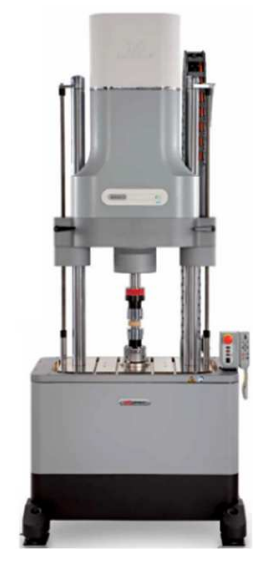

(a)

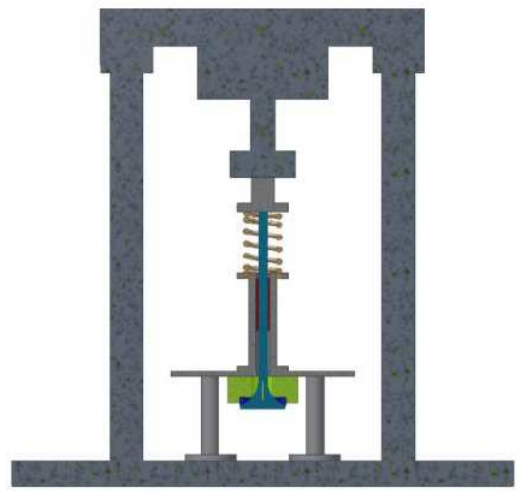

(c)

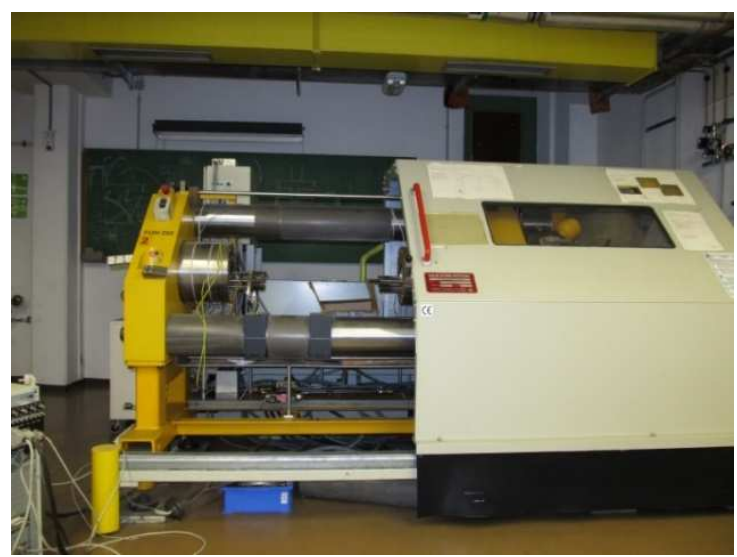

(b)

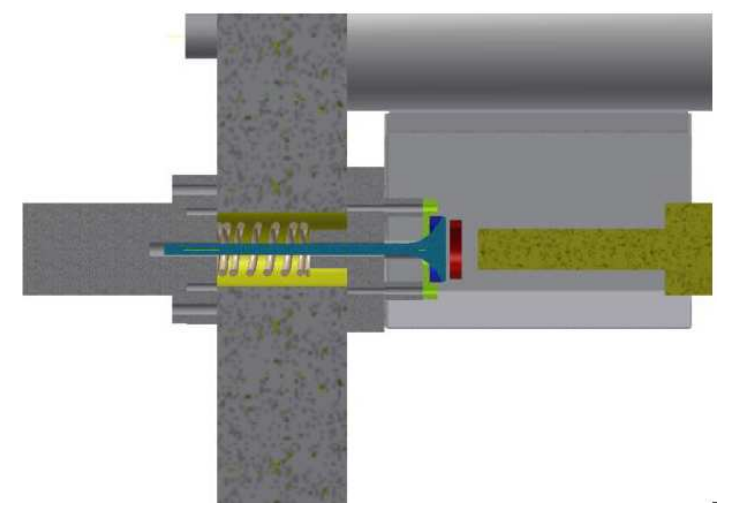

(d)

Abbildung 3.1: (a) elektrodynamische Universallösung, (b) servohydraulische Sonderlösung, CAD-Modelle möglicher Prüfaufbauten für (a) und (b) sind (c) bzw. (d)

Die elektrodynamische Universallösung ist interessant hinsichtlich der möglichen Einbaulage des Prüfsystems und Prüffrequenzen, da sie dem realen Betrieb entsprechen. Hingegen begrenzen Versuchsraum und darstellbare Prüfkräfte die Möglichkeiten. Das Potenzial der Sonderlösung entspricht bis auf die Einbaulage allen Anforderungen mit größter Übereinstimmung. Zur Einbaulage, Großmotoren werden als Reihen- oder V-Motoren gebaut. 
Aus Erfahrung des Autors kann im Feld in überwiegender Zahl ein höherer Ventilverschleiß für V-Motoren beobachtet werden. Betrachtet man vor diesem Hintergrund die vertikale Einbaulage in der Sonderlösung als Extremfall eines V-Motors, so kann sich dieser Umstand sogar als positiv herausstellen, da möglicherweise neue Erkenntnisse zum Verschleißverhalten des Tribosystems VS/SR gewonnen werden können. Darüber hinaus können elastizitätsbedingte Einflüsse durch den steifen Prüfrahmen der Sonderlösung minimiert werden. In Tabelle 3.1 sind die Auswahlkriterien für Universal- und Sonderlösung wiedergegeben.

Tabelle 3.1: Auswahlkriterien für Universal- und Sonderlösung

\begin{tabular}{lcc}
\hline & Universallösung & Sonderlösung \\
\hline Einbaulage des Ventils & vertikal & horizontal \\
Ventilrotation & ja & ja \\
Versuchsraum & + & +++ \\
Prüfkräfte & $\leq 10 \mathrm{kN}$ & $<160 \mathrm{kN}$ \\
Schließgeschwindigkeit & $<0,5 \mathrm{~m} / \mathrm{s}$ & $<1,2 \mathrm{~m} / \mathrm{s}$ \\
Prüffrequenz & $\leq 100 \mathrm{~Hz}$ & $\leq 10 \mathrm{~Hz}$ \\
Ventilmodifikation & ja & nein \\
Hochtemperatur & - & +++ \\
Hub & $\leq 60 \mathrm{~mm}$ & $\leq 30 \mathrm{~mm}$ \\
\hline
\end{tabular}

Der Umstand, dass bei Verwendung eines Stempels, die Kräfte über ein Koppelelement in die VS eingleitet werden sollen, führte zur Anforderung einer spielfreien, koaxialen Verbindung zwischen Stempel und VS. Für die Realisierung kam ein Passstift zwischen Koppelelement und VS mit entsprechend dimensionierter Übergangspassung zur Gewährleistung der Koaxialität und somit querkraftfreien Krafteinleitung zur Anwendung. Antrieb und Steuerung bestimmen die Leistungsfähigkeit einer Prüfmaschine. Typische Auswahlkriterien sind:

- Bewegungsform: Der Antrieb muss eine dreiecks- und sinusförmige Bewegung ermöglichen, Dreiecksform im Prüfmodus I und Sinusform im Prüfmodus II.

- Richtung: Der zu verwendende Antrieb muss einfachwirkend sein.

- Lastspielzahl: Der Antrieb muss für wiederholte Belastungen ausgelegt sein, um Zyklenzahlen in der Größenordnung von $10^{7}$ durchführen zu können.

- Höchstbelastung: Antriebsseitig sind Lasteinleitungen von bis zu 260 bar bei einem Tellerdurchmesser von ca. $90 \mathrm{~mm}$ zu realisieren.

- Arbeitshub: Vom Antrieb sind Hübe von bis zu $25 \mathrm{~mm}$ zu ermöglichen.

- Programmierbarkeit: Die Steuerung muss eine automatische Versuchsdurchführung für beide Prüfmodi von bis zu $1000 \mathrm{~h}$ ermöglichen.

Vom Antrieb einer Prüfmaschine sind nach [183] bestimmte Bedingungen hinsichtlich der Belastung zu erfüllen. Als wesentlich für einen VVP werden die folgenden drei angesehen. Der 
Antrieb der Prüfmaschine muss die Belastung auf den Prüfling von Null aus ansteigend bis zu einem Höchstwert möglichst ohne Stoßwirkung und stufenlos einleiten können. Die auf den Prüfling einzuleitende Last muss in bestimmten Stufen wählbar und möglichst ohne Stoßwirkung aufzubringen sein. Lastzyklen müssen in ihrem zeitlichen Ablauf steuerbar sein. Die Einstellbarkeit der für die Belastung der Probe vorgesehenen mechanischen Arbeit (z.B. Pendelschlagwerke) und die Steuerbarkeit der schwingenden Belastung zwischen beliebigen Grenzwerten (z.B. Dauerschwingprüfmaschinen) sind nicht erforderlich. Von den nach [185] sechs etablierten Aktuatoren, mechanisch, Resonanz, elektromechanisch, servopneumatisch, elektrodynamisch und servohydraulisch, werden folgend die für den VVP drei wichtigsten kurz vorgestellt:

Hydraulische Antriebe: Als hydraulisch wird ein Antrieb bezeichnet, wenn das energietragende Medium eine im Wesentlichen inkompressible Flüssigkeit, meist Hydrauliköl, ist. In der Hauptsache wird heute zwischen servohydraulischem Antrieb und servohydraulischem Resonanzantrieben unterschieden. Der servohydraulische Antrieb ist ein Konzept, das über eine breite Programmierbarkeit und hohe Flexibilität verfügt. Kräfte bis $10 \mathrm{MN}$ und Frequenzen bis $200 \mathrm{~Hz}$ sind realisierbar. Der hohe Energieverbrauch verursacht vergleichsweise hohe Versuchskosten. Man unterscheidet zwischen einfach- und doppeltwirkender Servohydraulik.

Elektromechanische Antriebe: Als elektromechanisch bezeichnet man einen Antrieb, der hauptsächlich aus mechanischen Elementen besteht, die aber durch einen elektrischen Primärantrieb in Bewegung gesetzt werden. Ein Regelverstärker verarbeitet die vom Sollwertund Istwert-Geber kommenden Signale und leitet das Ergebnis an den Antriebsmotor weiter. Dieser bewirkt über Zwischenglieder eine Krafteinleitung in der Weise, dass der Istwert dem Sollwert folgt.

Elektromagnetische Antriebe: Als elektromagnetisch wird ein Antrieb bezeichnet, bei dem die von einem Elektromagneten auf einen Anker oder stromdurchflossenen Leiter wirkende Kraft ohne Zwischenglieder zur Bewegung verwendet wird. Bei genügend kleinem Luftspalt kann mit einem Elektromagneten eine relativ große Anziehungskraft auf einen in dessen Feld lokalisiertem Anker ausgeübt werden. Daher ist der elektromagnetische Antrieb für hochfrequente Prüfungen mit kleinen Hüben die bevorzugte Wahl.

Ein elektromechanischer Antrieb bietet Vorteile hinsichtlich seines einfachen Aufbaus mit Spindelantrieb oder Linearmotor. Hohe Prüfkräfte bis $150 \mathrm{kN}$ und lange Hübe können nicht realisiert werden, hingegen ist eine Bandbreite von 0 bis ca. $30 \mathrm{~Hz}$ an Prüffrequenzen sowie beliebigen Signalformen umsetzbar. Elektromagnetische Antriebe sind interessant für höhere Prüffrequenzen bis $300 \mathrm{kHz}$. Die darstellbaren Hübe von wenigen Millimetern und Prüfkräfte begrenzen seine Anwendbarkeit. Die oben beschriebenen Möglichkeiten des servohydraulischen Antriebs bilden für die zu entwickelnde Prüfmaschine einen Schwerpunkt. Im Hinblick auf Kraft und Hub sind mechanische Antriebe interessant. Da bei mechanischen Aktuatoren Weg- und Kraftänderungen einen Umbau bedingen und nur ein sinusförmiger Kraftverlauf möglich ist, fiel die Entscheidung auf einen servohydraulischen Antrieb. In Tabelle 3.2 ist eine Bewertung der Auswahlkriterien für alle Aktuatoren wiedergegeben. 
Tabelle 3.2: Bewertung der Auswahlkriterien für Aktuatoren

\begin{tabular}{lccccc}
\hline Aktuator & Kraft & Hub & Frequenz & Signale & Kosten \\
\hline mechanisch & ++ & +++ & - & Sinus & - \\
Resonanz (mechanisch) & ++ & + & + & Block & - \\
Resonanz (magnetisch) & + & -- & ++ & Block & - \\
Elektromechanisch & + & - & - & BF & ++ \\
Servopneumatisch & - & ++ & - & BF & ++ \\
Elektrodynamisch & ++ & - & +++ & BF & ++ \\
Servohydraulisch & +++ & +++ & ++ & BF & +++ \\
\hline
\end{tabular}

Eine Lasteinleitung über die Tellerplanfläche mittels Hydraulikstempel bewirkt im Vergleich zum Gasdruck bei Anwendung einer druckäquivalenten Kraft eine andere Tellerdurchbiegung. Daher wurden zur Dimensionierung des Stempeldurchmessers Berechnungen mittels Finite Element Methode (FEM) durchgeführt. Als Referenz für die Durchbiegung wurde der Gasdruck definiert. Im ersten Schritt erfolgte eine Parameterstudie mit unterschiedlich feinen Netzen zur Festlegung einer geeigneten Vernetzung für die FEM-Berechnungen. In Abbildung 3.2 sind die Durchbiegungen in Ventilachsrichtung für die drei Netzgrößen 50, 25 und $10 \mu \mathrm{m}^{2}$ dargestellt. Die ermittelten Durchbiegungen sind mit $49 \mu \mathrm{m}$ in allen Fällen gleich groß.

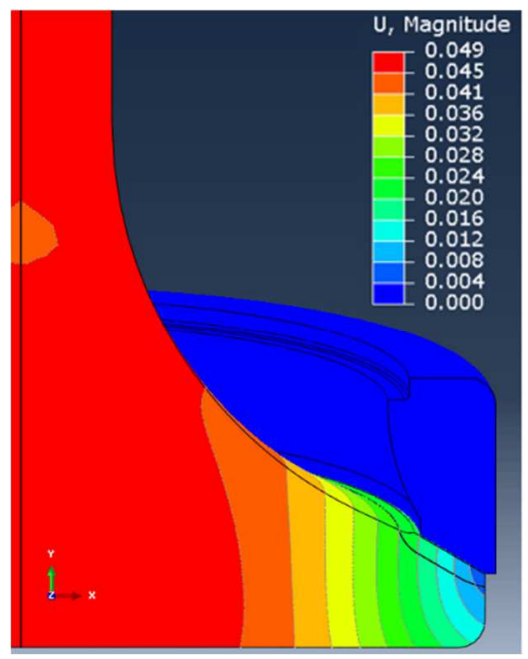

Mesh 1: $50 \mu \mathrm{m}^{2}$

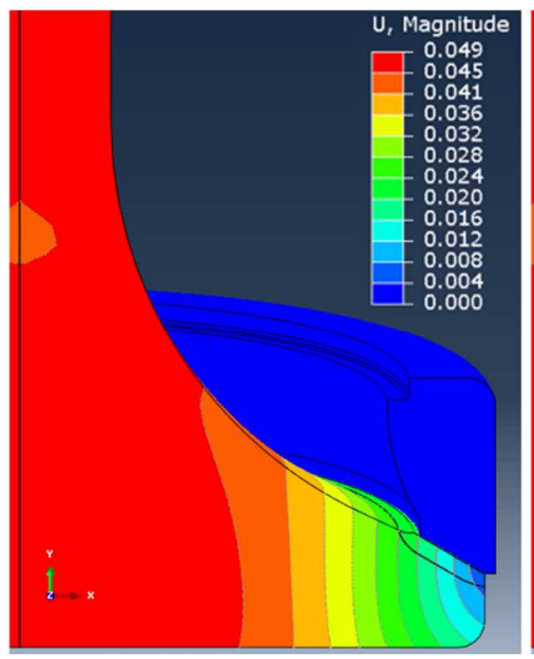

Mesh 2: $25 \mu \mathrm{m}^{2}$

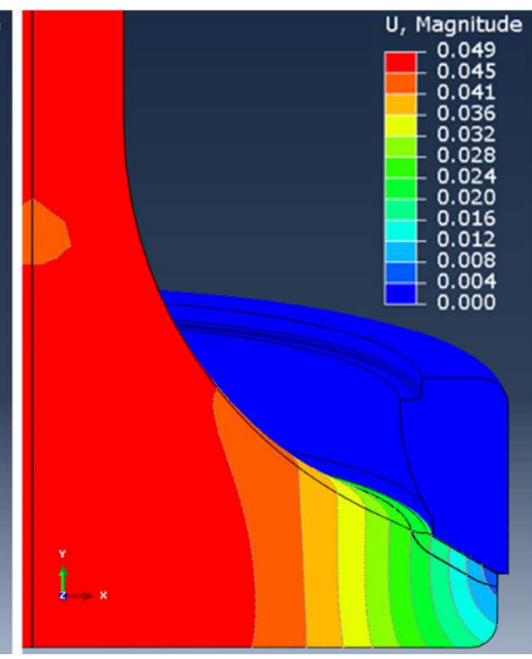

Mesh 3: $10 \mu \mathrm{m}^{2}$

\section{Abbildung 3.2: Parameterstudien zur Vernetzung}

Im nächsten Schritt wurden die Durchbiegungen von experimenteller Simulation und Gasdruck aufeinander abgestimmt. Abbildung 3.3 veranschaulicht beispielhaft die Ergebnisse zur Durchbiegung infolge Stempelkraft und Gasdruck. Somit werden während der experimentellen Simulationen zum Verbrennungsdruck annähernd gleiche Durchbiegungen zwischen VS und SR im Vergleich zum Motorbetrieb erreicht. 


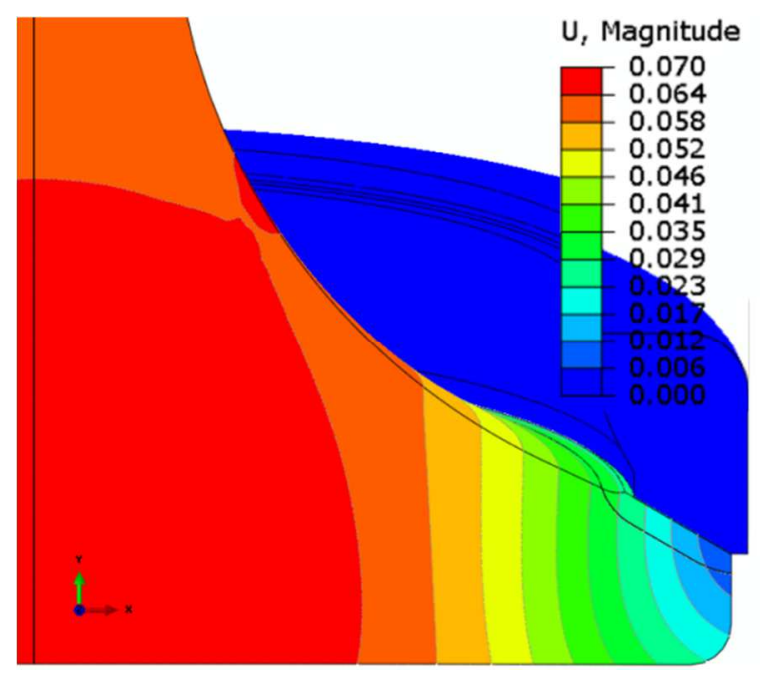

(a)

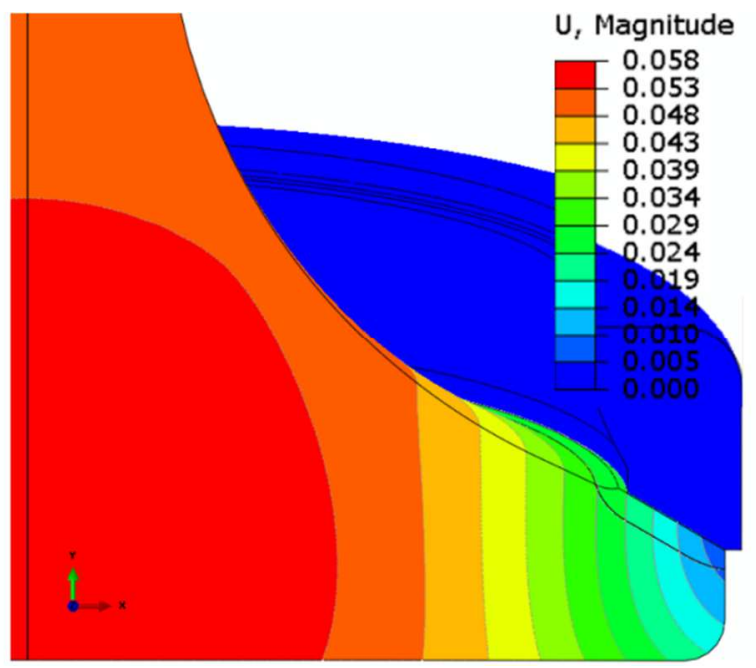

(b)

Abbildung 3.3: Vergleichende Finite Element Analysen zur Tellerdurchbiegung infolge Stempelkraft (a) und Gasdruck (b)

\subsubsection{Prüfmodus I und II}

Für die experimentellen Simulationen sind bei Frequenzen bis $10 \mathrm{~Hz}$ im Prüfmodus I Schließgeschwindigkeiten bis $1,2 \mathrm{~m} / \mathrm{s}$ und im Prüfmodus II Verbrennungsdrücke bis 260 bar gefordert. Als Referenz wurden Versuche bei $380{ }^{\circ} \mathrm{C}$ an Luft unter $0,6 \mathrm{~m} / \mathrm{s}$ und 180 bar definiert. Neben den beiden Hauptparametern sollen später Temperatur und Atmosphäre kontrolliert variiert werden können. Beide Prüfmodi sollen möglichst einflussfrei vom jeweils anderen untersucht werden können. Das gewählte Tribosystem VS/SR stammt aus der Einlassseite eines Großgasmotors mit einer Motordrehzahl von $1000 \mathrm{~min}^{-1}$. Somit ergibt sich eine Prüffrequenz von 8,3 Hz für beide Prüfmodi. Die im Kapitel 2.3 ermittelte Verschleißrate von $3,45 \mathrm{~mm}^{3} / \mathrm{h}$ ist aus Erfahrung des Autors untypisch hoch für EVSn. Daher fokussieren sich die Untersuchungen in dieser Arbeit auf das Verschleißverhalten des einlassseitigen Tribosystem VS/SR. Grohe nennt in [110] bis zu $500^{\circ} \mathrm{C}$ für EVSn. Aus Erfahrung des Autors sind in überwiegender Zahl Temperaturen um die $380^{\circ} \mathrm{C}$ für EVSn in Großgasmotoren typisch. Daher wurde für spätere Versuchsreihen eine 3-stufige Variation von 330,380 und $430^{\circ} \mathrm{C}$ definiert. Die Wahl einer adiabatischen Erwärmung durch komprimierte Luft mittels Kolbenmaschine wäre ein anwendungsnaher Ansatz, wird jedoch aufgrund der oben genannten Gründe nicht weiter betrachtet. Aus diesem Grund wurde als zuverlässige und robuste Wärmequelle eine Induktionsheizung gewählt. Unter dem Aspekt zukünftiger Versuchsreihen wurde für das Heizsystem eine Maschinenfähigkeit von maximal $900^{\circ} \mathrm{C}$ vereinbart. Tabelle 3.3 gibt eine Übersicht des definierten Parameterkennfelds wieder. 
Tabelle 3.3: Übersicht des Parameterkennfelds für den VVP bezogen auf einen Tellerdurchmesser von ca. $90 \mathrm{~mm}$ bei einer Masse von ca. $1300 \mathrm{~g}$

\begin{tabular}{|c|c|c|c|}
\hline Parameter & Einheit & $\begin{array}{c}\text { Prüfmodus I, } \\
\text { Ventilschließen }\end{array}$ & $\begin{array}{c}\text { Prüfmodus II, } \\
\text { Verbrennungsdruck }\end{array}$ \\
\hline Schließgeschwindigkeit & {$[\mathrm{m} / \mathrm{s}]$} & $0,1-1,2$ & $\leq 0,1$ \\
\hline Prüfkraft & {$[\mathrm{kN}]$} & $1-10$ & $\leq 150$ ( $\equiv 260$ bar $)$ \\
\hline Ventilhub & {$[\mathrm{mm}]$} & $\leq 25$ & $\leq 10$ \\
\hline Prüffrequenz & {$[\mathrm{Hz}]$} & $\leq 10$ & $\leq 8,3$ \\
\hline Temperatur & {$\left[{ }^{\circ} \mathrm{C}\right]$} & \multicolumn{2}{|c|}{ RT - 900} \\
\hline
\end{tabular}

Im Hinblick auf Vergleichbarkeit der Prüfergebnisse wurde bei der Konstruktion eine Gleichteilestrategie angewendet, d.h. möglichst viele gleiche Komponenten sollen in beiden Prüfmodi zur Anwendung kommen. Daneben ergibt sich aus diesem modularen Bausystem auch eine Kosteneffizienz. Eine Neupositionierung von VS und SR infolge Probenentnahme zur intervallmäßigen Messung des Verschleißprofils sollte vermieden werden. Daneben war ein einfacher Ein- und Ausbau von VS und SR wichtig, um Stillstandszeiten bei der Versuchsdurchführung zu minimieren. Auch im Fall von Störungen ist eine einfache Handhabung vorteilhaft. Zur Veranschaulichung des Konstruktionsprozesses ist exemplarisch in Abbildung 3.4 eine Mind Map für Prüfmodus II dargestellt, welche Teil der Konzeptphase war. Die Festforderungen für die Konstruktion sind rot eingerahmt.

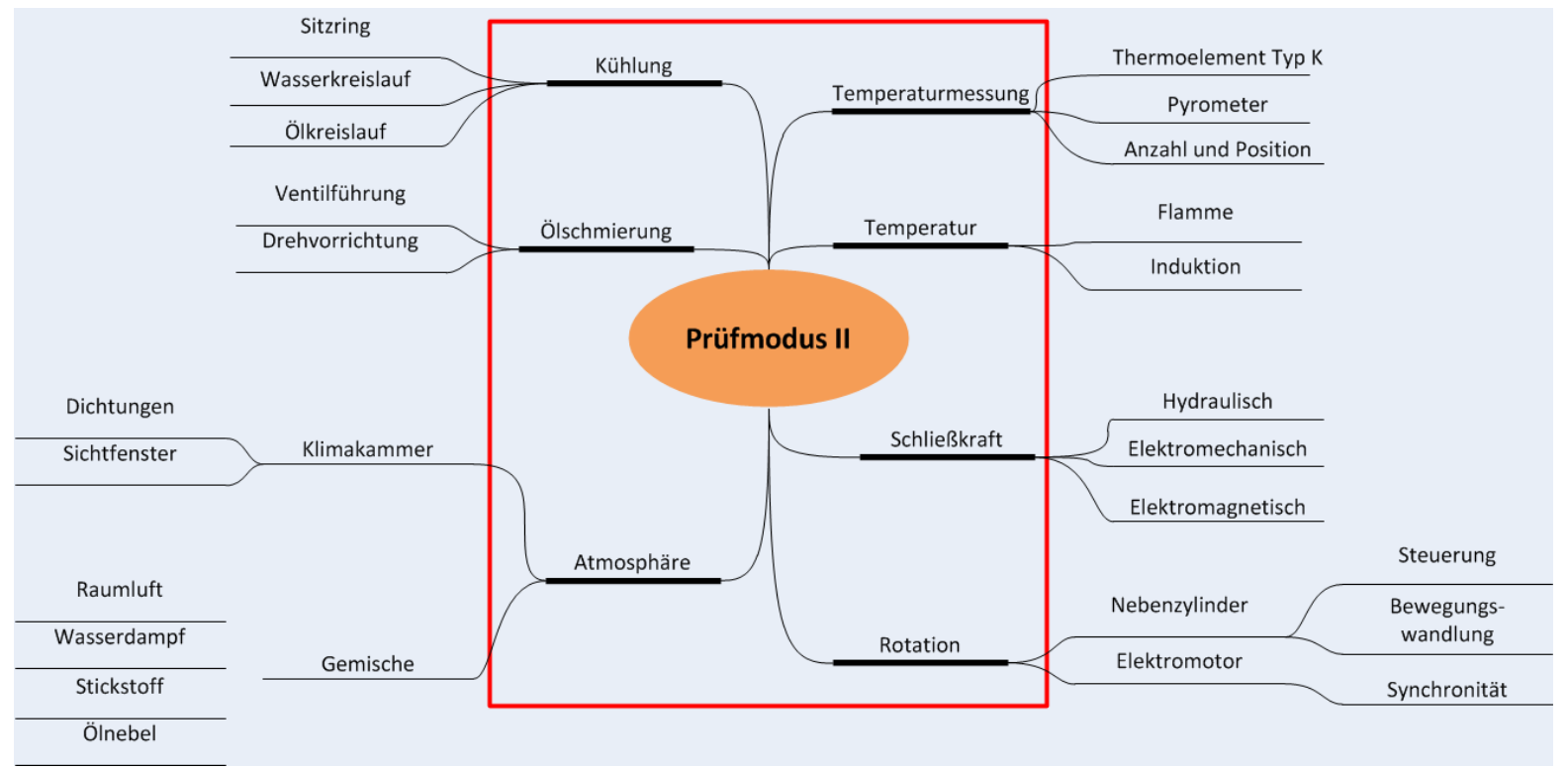

\section{Abbildung 3.4: Mind Map als Teil der Konzeptphase von Prüfmodus II}

Während der Detallierung des Prüfstandskonzepts in Unterbaugruppen wurde deutlich, dass für Prüfmodus II ein anderer Ventildrehmechanismus im Vergleich zu Modus I erforderlich wird. Die Anforderungen zur Verwendung der originalen Ventildrehvorrichtung und Schließgeschwindigkeiten bis $1,2 \mathrm{~m} / \mathrm{s}$ konnten im Prüfmodus I mit einem weggeregelten 
Hydraulikzylinder umgesetzt werden. Hingegen können die Anforderungen hoher Prüfkräfte bis $150 \mathrm{kN}$ und Ventilhübe bis $25 \mathrm{~mm}$ bei Verwendung der originalen Drehvorrichtung im kraftgeregelten Betrieb von Prüfmodus II nicht zusammengeführt werden. In der Folge wurde die Konstruktion eines neuen Drehmechanismus erforderlich. Als Aktuator konnte der Hydraulikzylinder aus Prüfmodus I in den Mechanismus integriert werden.

Im Ergebnis wurde ein Prüfstandskonzept erarbeitet, mit dem die experimentellen Untersuchungen zu den Ventilphasen Schließen und Verbrennungsdruck getrennt bei der geforderten Prüffrequenz bis $10 \mathrm{~Hz}$ ermöglicht werden konnte. In Abbildung 3.5 ist eine schematische Darstellung der beiden Prüfmodi veranschaulicht.

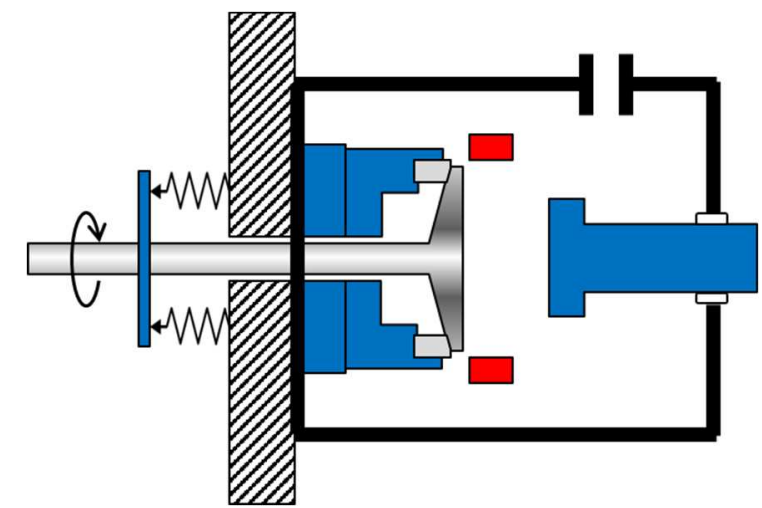

(a)

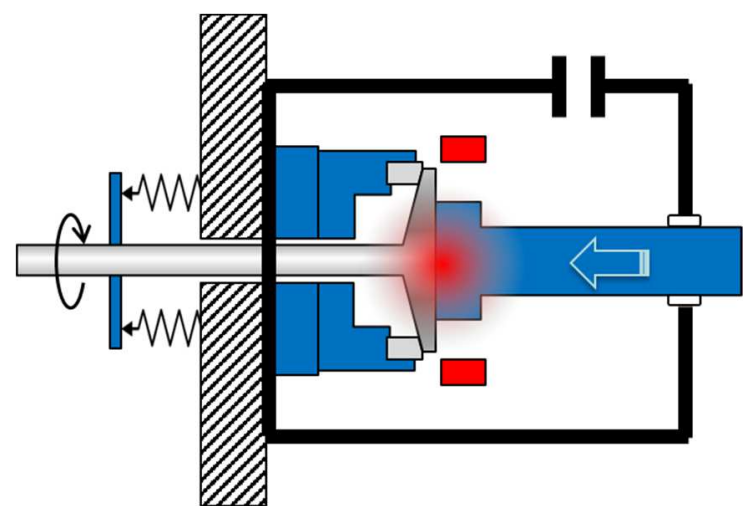

(b)

Abbildung 3.5: Schematische Darstellung von Prüfmodus I (a) und Prüfmodus II (b)

\subsection{Aufbau des Ventilverschleißprüfstands}

\subsubsection{Prüfgestell}

Das verwendete Prüfgestell des VVPs basiert auf einem $250 \mathrm{kN}$ steifen Prüfrahmen mit zwei massiven Jochen an den Außenseiten verbunden durch zwei Rundrohre mit Durchmesser $180 \mathrm{~mm}$. Die hohe Steifigkeit wurde gewählt, um den negativen Einfluss von auftretenden Elastizitäten und Verformungen des Prüfrahmes während der Versuche insbesondere bei hohen Prüfkräften bis $150 \mathrm{kN}$ im Prüfmodus II zu minimieren. Ursprünglich wurde diese Prüfmaschine für Innenhochdruckversuche von Aluminiumrohren mit einer horizontalen Prüfachse verwendet, wobei ein Hydraulikzylinder in einem abgeschlossenen Rohr den Hochdruck erzeugte. Dieser Umstand erklärt die horizontale Prüfanordnung. Abbildung 3.6 (a) veranschaulicht den ausgewählten Prüfrahmen vor dem Aufbau. Aus Gründen des Arbeitsumfangs sollen an dieser Stelle nur die wichtigsten Meilensteine des Aufbaus genannt werden. In einem ersten Schritt wurde das Prüfgestell vollständig für eine Bestandsaufnahme demontiert. Nach Vereinbarung der Aufbauarbeiten erfolgte die vollständige Instandsetzung der Hydraulikleitungen. Anschließend wurden die Modifikationen an den Jochen zur Montage der Prüfaufbauten durchgeführt. Neben dem vollständigen Austausch der hydraulischen Verrohrung aufgrund von Ablagerungen und Korrosion wurden neue Steuerungskomponenten wie Hydraulikventile verbaut. 


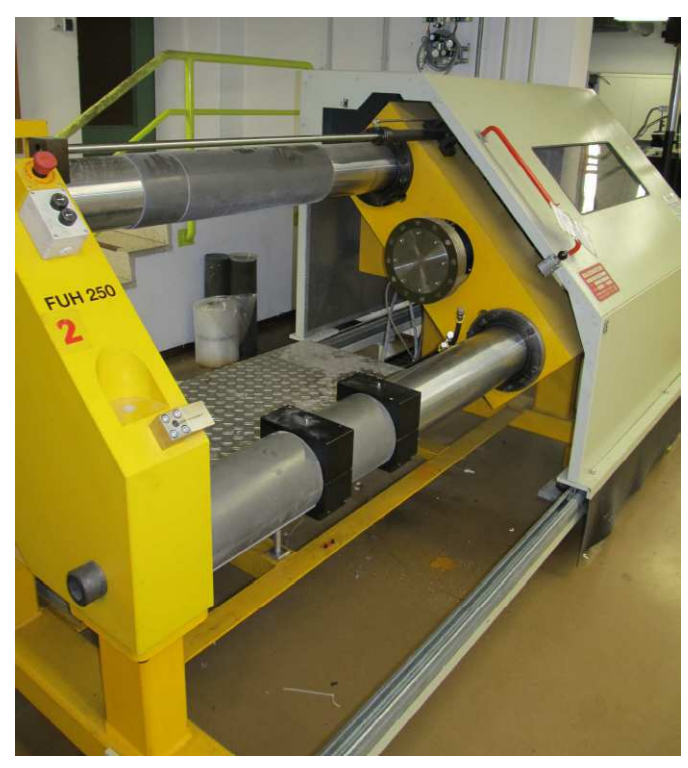

(a)

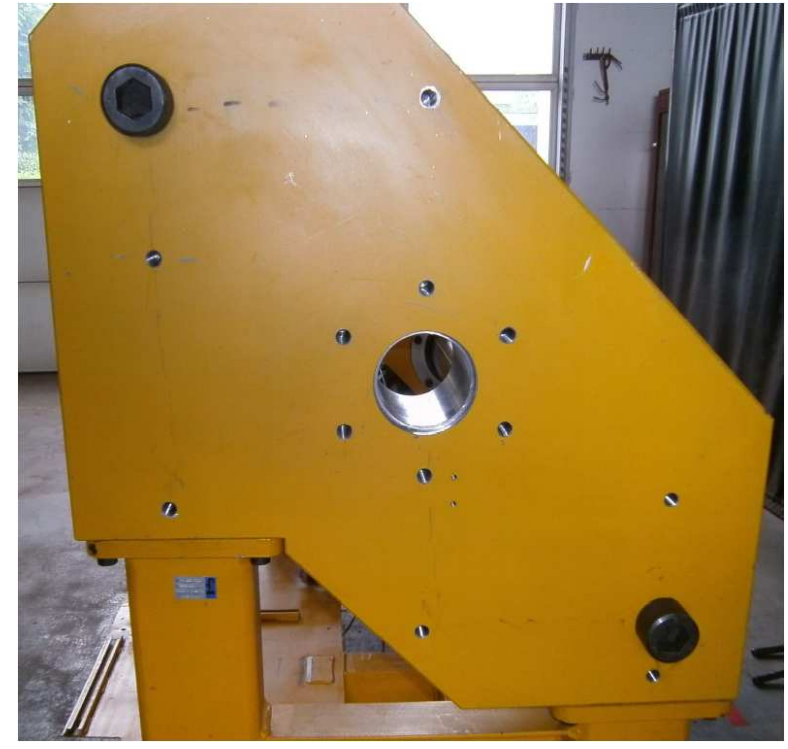

(b)

Abbildung 3.6: (a) Prüfrahmen vor Aufbau, (b) Modifikation des linksseitigen Jochs

Abbildung 3.7 veranschaulicht den Prüfstand vor und nach der Instandsetzung. Darüber hinaus wurde der Schallschutz nach dem Umbau blau lackiert, in seiner Tiefe für einen Raumgewinn zur Installation der Induktionsheizung vergrößert und die Elektrik im Verbund mit den Hydraulikkomponenten den aktuellen Richtlinien angepasst. Ein wichtiger Punkt war die Modifikation des linksseitigen Maschinenjochs für die Montage des Prüfaufbaus im Modus I. Abbildung 3.6 (b) stellt die Außenseite des überarbeiteten Maschinenjochs dar. Nach dem Herstellen einer zentralen Durchgangsbohrung im Joch wurden stirnseitig Übermaßpassungen zur Ausrichtung der später anzuschraubenden Flansche gefertigt. Hierbei wurde besonders auf Koaxialität geachtet, um spätere Fluchtungsfehler im Prüfaufbau zu vermeiden. Konzentrisch zur Durchgangsbohrung wurden auf der Außenseite sechs Stück Gewinde M12x50 auf einem Teilkreis von 200 mm zur Befestigung der Prüfaufbauflansche in das Joch geschnitten.

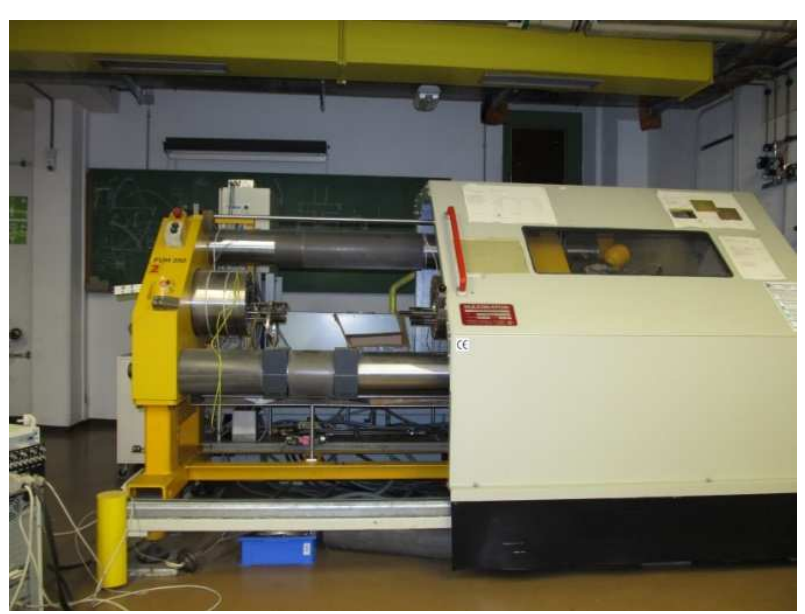

(a)

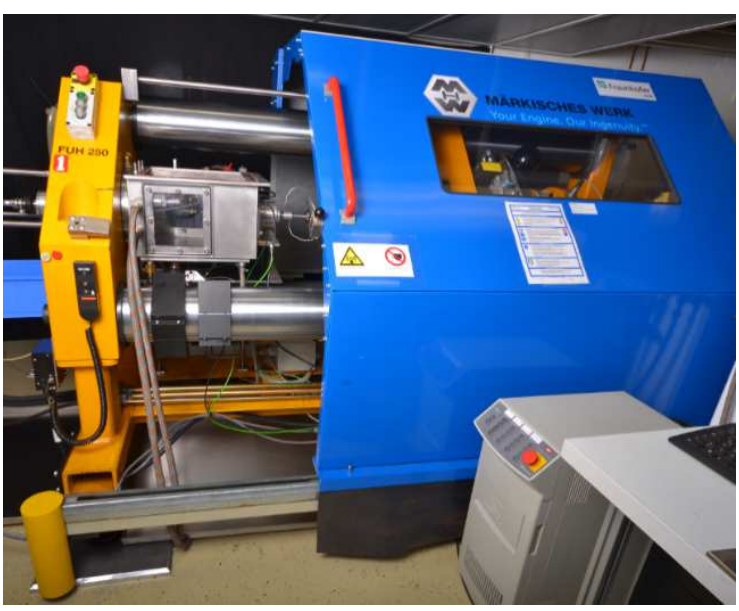

(b)

Abbildung 3.7: Prüfrahmen vor (a) und nach (b) der Instandsetzung 


\subsubsection{Tastversuche zur Induktionsheizung}

Wesentliche Komponenten einer induktiven Heizung sind Generator und Induktor. Der Induktor transferiert das vom Generator erzeugte magnetische Wechselfeld auf den ferromagnetischen Werkstoff, induziert Wirbelströme, die zu einer Erwärmung des Bauteils führen. Der Induktor wurde als einschleifige Spule ausgeführt. Abbildung 3.8 (a) stellt den Versuchsaufbau dar. Erkennbar sind die drei Komponenten Ventilteller (1), Induktor (2) und die applizierten Thermoelemente (3). Der Induktor wurde mit einem Koppelabstand von $1 \mathrm{~mm}$ an der Tellerplanseite montiert.

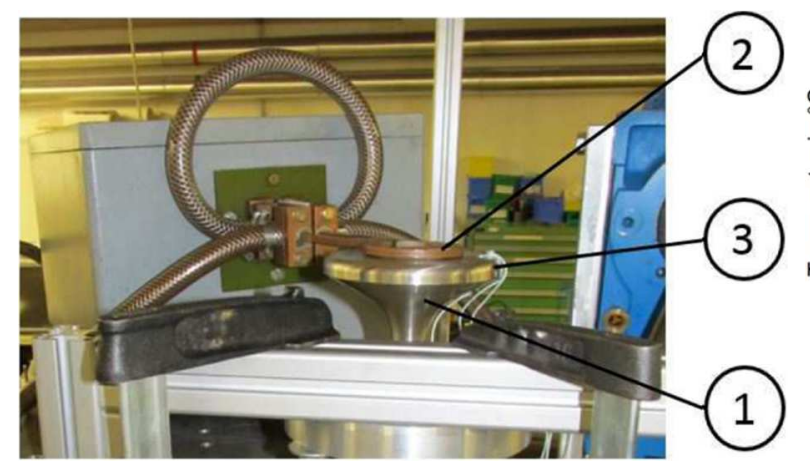

(a)

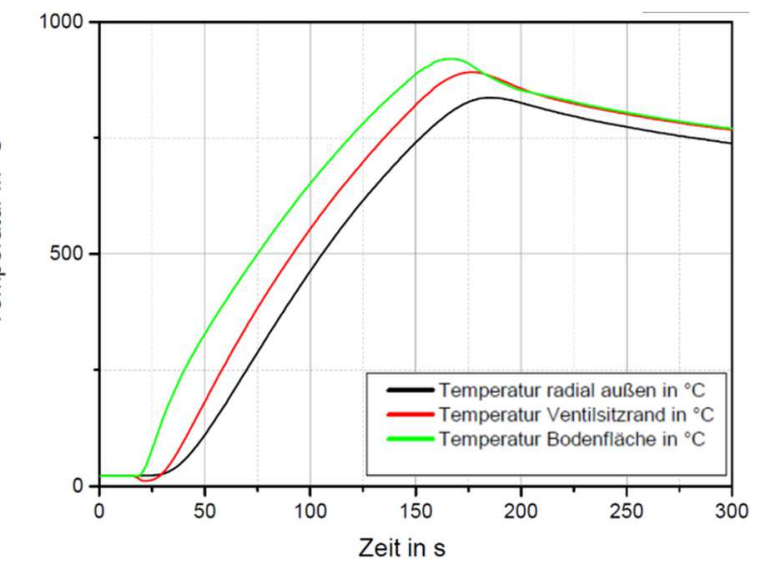

(b)

Abbildung 3.8: (a) Versuchsaufbau der Tastversuche zur induktiven Heizung, (b) Temperatur-Zeitverlauf am Ventilteller an den drei Positionen Tellermitte (grün), Tellerdurchmesser (schwarz) und SID (rot) bei maximaler Generatorleistung

In einem ersten Schritt wurden Tastversuche mit einer am Fraunhofer IWM vorhandenen Induktionsheizung zur Leistungsüberprüfung des Hochfrequenzgenerators durchgeführt (Abbildung 3.9). Die Auswertung der Temperaturverläufe über die Zeit bei maximaler Generatorleistung ist in Abbildung 3.8 (b) dargestellt. Nach einer Zeit von $160 \mathrm{~s}$ wurde eine Temperatur von $900{ }^{\circ} \mathrm{C}$ an der Tellerplanfläche erreicht.

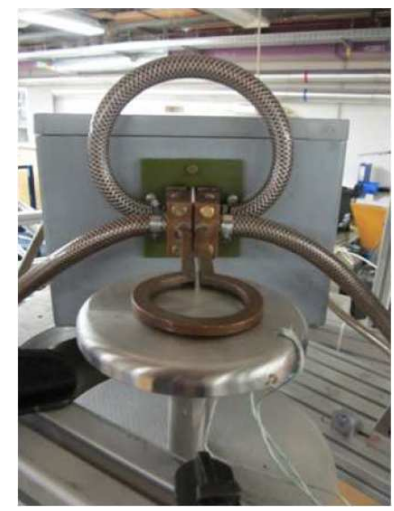

(a)

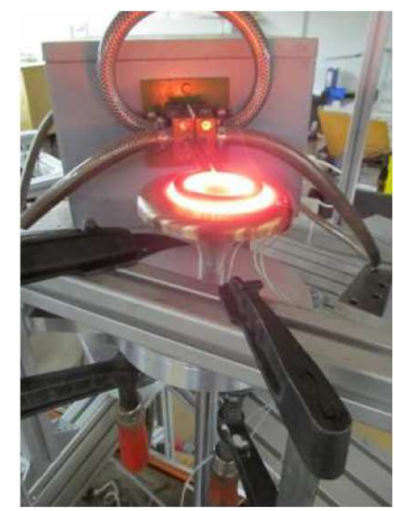

(b)

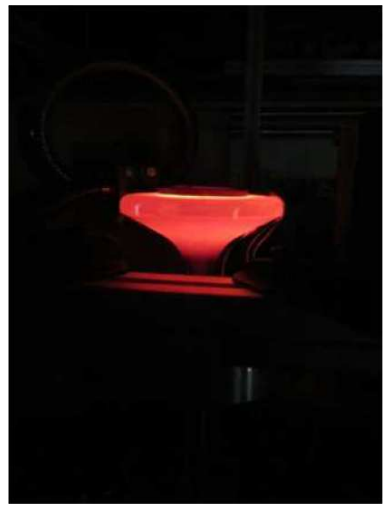

(c)

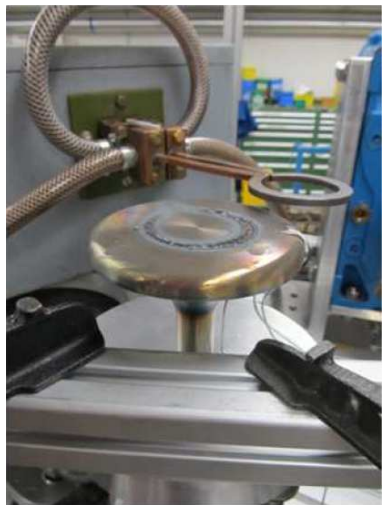

(d)

Abbildung 3.9: Vorversuche zur Induktionsheizung 
Eine erfolgreiche Validierung der Maschinenfähigkeit Generator konnte somit nachgewiesen werden. In Bezug auf die geforderte maximale Versuchstemperatur von $450{ }^{\circ} \mathrm{C}$ ist die Heizleistung ausreichend und die Versuchsanordnung robust. In Abbildung 3.9 (d) sind lokale Anschmelzungen an der Tellerplanseite erkennbar, die auf eine zu hohe Heizleistung oder zu kleinem Induktor zurückzuführen sind. Bei der späteren Versuchsdurchführung wurde ein größerer Induktor verwendet.

\subsubsection{Gasdichte Klimakammer}

Für die Simulation des Einflusses verschiedener Atmosphären wurde eine gasdichte Klimakammer konstruiert. Bei der in Abbildung 3.10 veranschaulichten kühlbaren Klimakammer handelt es sich um eine Blechkonstruktion aus Edelstahl mit gasdicht umlaufenden Schweißnähten.
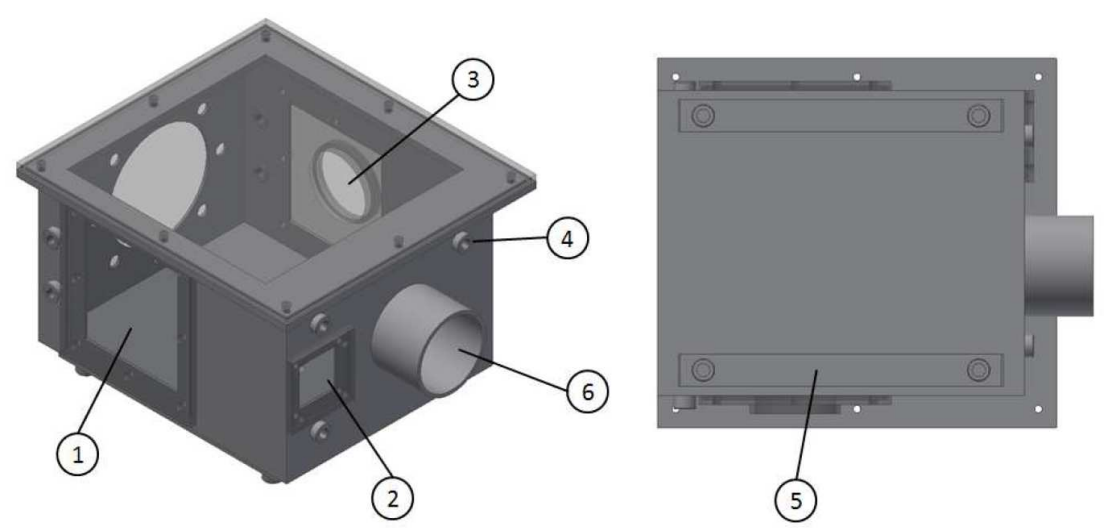

\section{Abbildung 3.10: CAD-Modell der Klimakammer}

Transparent dargestellt ist das große Plexiglasfenster (1) zur visuellen Beobachtung der Versuche. Das kleine Fenster (2) schafft die Möglichkeit für eine berührungslose Temperaturmessung von außen mittels Pyrometer. Fenster (3) dient dem Zugang des Induktors in die Kammer. Nach Vorversuchen mit Polymethylmethacrylat (PMMA) wurde Polycarbonat (PC) als Werkstoff für die Sichtfenster gewählt, welches eine Temperatur bis $135^{\circ} \mathrm{C}$ in der Kammer ermöglicht. Ein eingeklebter Ring dient als Stutzen zur Befestigung eines Faltenbalgs, um an dieser Schnittstelle Gasdichtigkeit zu gewährleisten. Die eingeschweißten Gewindezapfen (4) schaffen die Möglichkeit Gasleitungen anzuschließen. Optional könnte auch Ölnebel als weitere Variation der Atmosphäre eingeleitet werden. An der Unterseite sind Rechteckrohre (5) mit Gewindezapfen zur Kühlung der Kammer angeschweißt. Über den Blechstutzen (6) werden im Prüfmodus I die Signalleitungen der Thermoelemente nach außen geführt, hingegen ermöglicht die Öffnung im Prüfmodus II den Zugang des Hydraulikstempels zur Lasteinleitung an der Tellerplanseite. Ein montierter Faltenbalg sichert im Modus II gegen Gasleckage ab. Abbildung 3.11 (a) veranschaulicht die zusammengebaute Klimakammer als CAD-Modell. Die Kammer besteht im Wesentlichen aus der transparenten Polycarbonatplatte (1) und dem Aluminiumrahmen (2). Zur Abdichtung der Platte wird ein umlaufender Dichtring verwendet, für den eine Nut (3) in die Auflagefläche gefräst wurde. Abschließend erfolgte 
noch ein Planfräsen der Fläche. Für einen gleichmäßigen Anpressdruck wird die Platte mit 16 Stück M8-Schrauben auf dem Rahmen befestigt. Die Gasdichtheit der Klimakammer wurde gemäß DIN EN 1779 nachgewiesen.

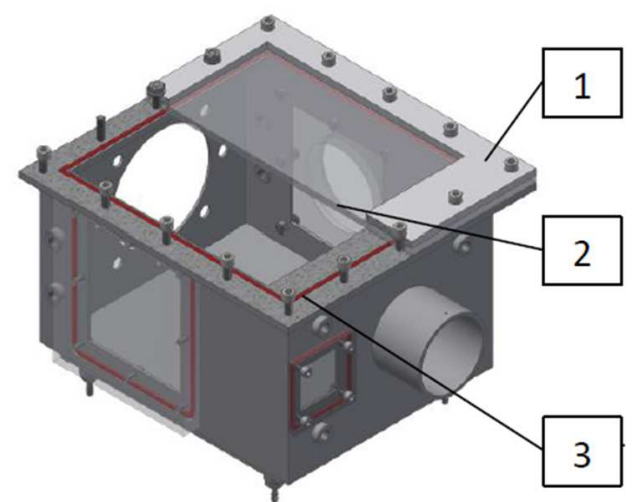

(a)

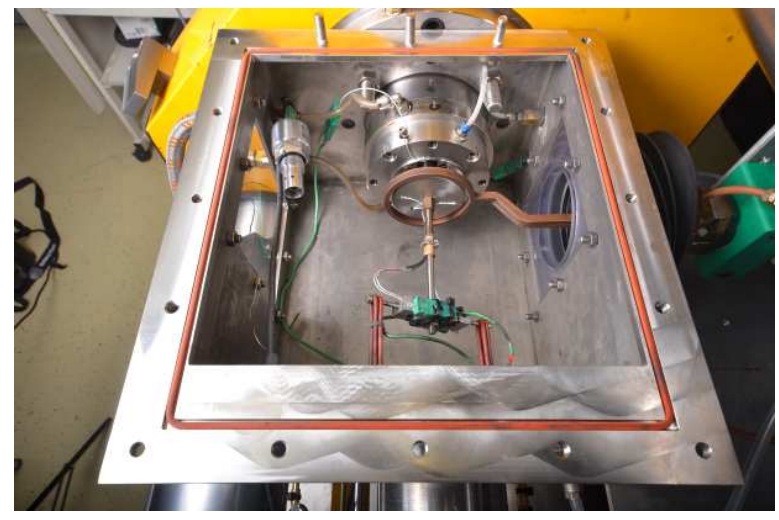

(b)

Abbildung 3.11: Klimakammer als CAD-Modell (a) und Fertigteil in Draufsicht (b)

\subsubsection{Prüfmodus I-Simulation des Ventilschließens}

Für die technische Umsetzung von Prüfmodus I war die Entwicklung einer Versuchsanordnung unter Verwendung der realen Komponenten des Ventilsystems die Vorgabe (vgl. Kapitel 3.1). Das Ventilsystem besteht aus VS, SR, Führung, Ventilfedern, Federteller, Klemmstücken und Drehvorrichtung. Der entwickelte Prüfaufbau wird am linksseitigen Joch montiert (vgl. Abbildung 3.6 (b)). Abbildung 3.12 veranschaulicht Prüfmodus I im Halbschnitt als CADModell. Eine Zusammenbauzeichnung mit Stückliste findet sich im Anhang (Abbildung 8.2).

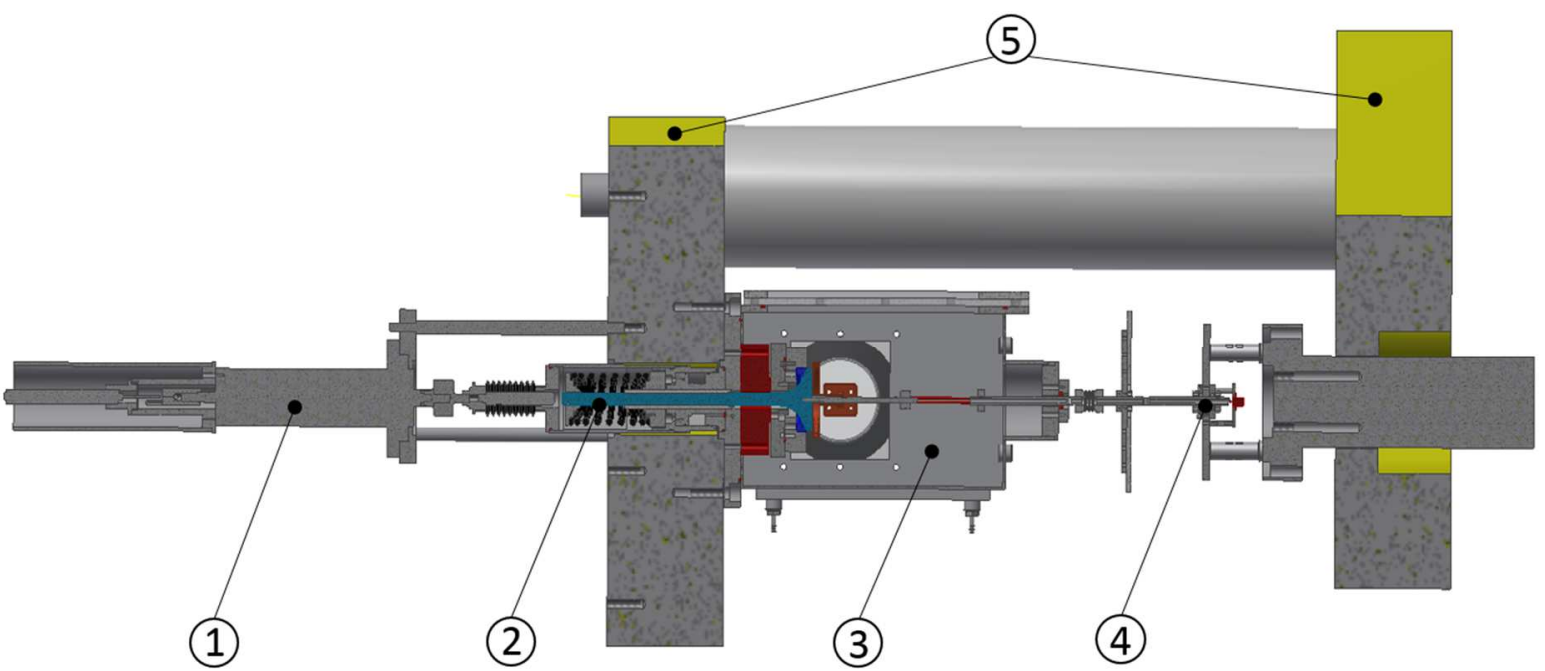

Abbildung 3.12: CAD-Modell von Prüfmodus I im Halbschnitt 
Die Prüfanordnung besteht aus den fünf Hauptbaugruppen Hydraulikzylinder (1), Ventilsystem (2), gasdichte Klimakammer (3), Schleifringüberträger (4) sowie Prüfrahmen (5). Aufgrund des Hauptparameters Ventilschließgeschwindigkeit wird der Hydraulikzylinder im Prüfmodus I weggeregelt betrieben. Die Schließkraft spielt nur eine untergeordnete Rolle. Die VS wird im Prüfmodus I oszillierend vom Zylinder aktuiert, indem ein Stößel auf das Schaftende der VS drückt. Infolge des Ventilhubs werden die Ventilfedern zusammengedrückt, wodurch die mechanische Ventildrehvorrichtung eine Ventilrotation während des Ventilöffnens bewirkt. Die verwendete originale Drehvorrichtung ermöglicht in Kombination mit einem simulierten Hub von $25 \mathrm{~mm}$ eine anwendungstypische Ventilrotation von ca. 11 Umdrehungen pro Minute. Die Hauptbaugruppe Ventilsystem (2) wird in der Durchgangsbohrung des linken Jochs positioniert, über die links- und rechtsseitigen Passungen der Durchgangsbohrung koaxial ausgerichtet und beidseitig am Joch verschraubt. Die prüfstandsspezifische Klimakammer wird innenseitig am linksseitigen Joch montiert. Abbildung 3.13 (a) veranschaulicht den Hydraulikzylinder mit Wegmesssystem am linken Maschinenjoch. Für einen kontrollierten Ventilhub wurde ein verfahrbarer Maschinentisch (1) konstruiert, auf dem der hydraulische Antriebszylinder (3) mit dem Wegmesssystem (2) linear verschoben werden kann. Die Versuche sollen bei kontrollierten Ventiltemperaturen durchgeführt werden. Daher wurde zur Regelung der Kühlkreisläufe und induktiven Heizung eine Temperaturüberwachung für VS und SR erforderlich. Jeweils zwei applizierte Thermoelemente an VS und SR übernehmen diese Aufgabe. Aufgrund der Ventilrotation war eine Transmissionsschnittstelle zu schaffen, um die Signalleitungen in ein kabelloses Signal zu transferieren. Die Leitungen werden über eine Hohlwelle aus der Klimakammer herausgeführt. Die Transmission des elektrischen Messsignals wurde durch einen Schleifringüberträger umgesetzt. Abbildung 3.13 (b) stellt den Aufbau im CAD-Modell dar. Die Messdatenerfassung erfolgte mittels LabVIEW.

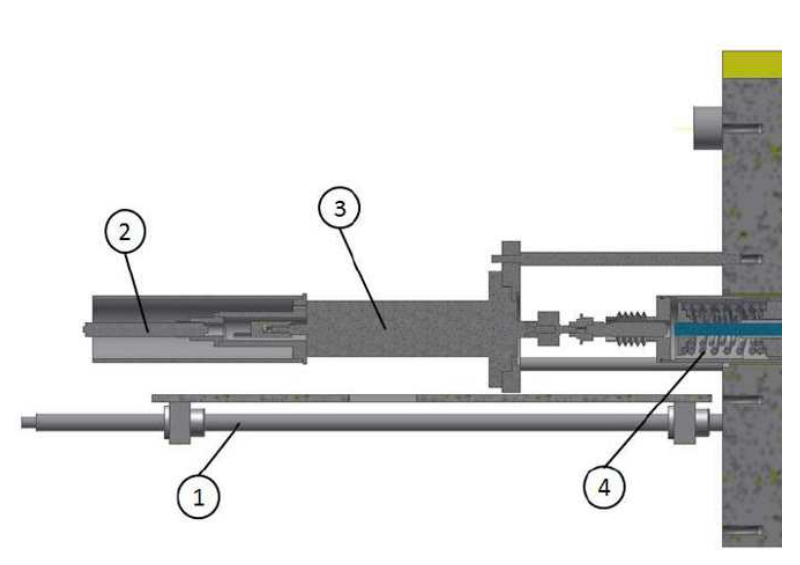

(a)

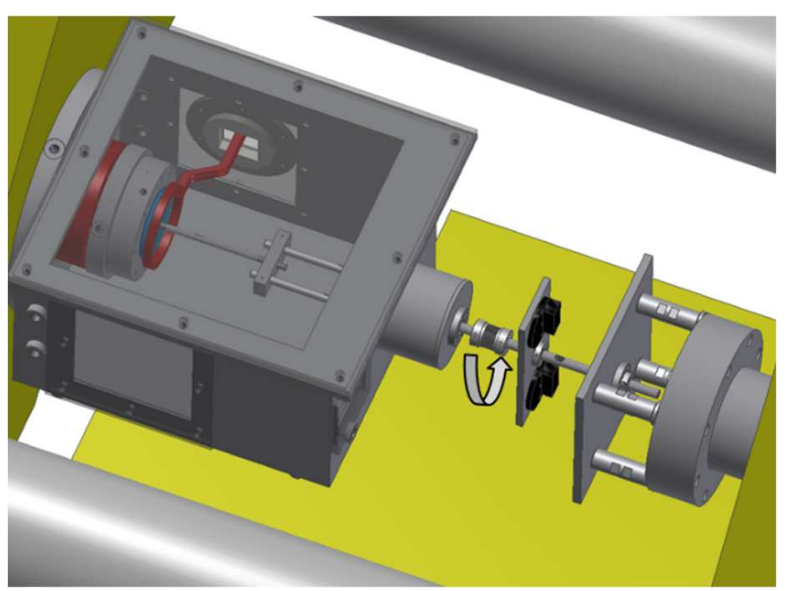

(b)

\section{Abbildung 3.13: CAD-Modelle des Hydraulikzylinders mit Wegmesssystem (a) und der Klimakammer mit Slip-Ring-Transmitter (b)}




\subsection{5 Ölschmierung und Kühlung}

Abbildung 3.14 stellt die Einbausituation von VS und SR hinsichtlich Ölschmierung und Kühlung dar. Neben dem Aspekt der Kühlung wurde zur Vermeidung von Reibung und Verschleiß an Ventilfedern und Drehvorrichtung ein Ölbad im Hohlzylinder (1) integriert. Kompression und Dekompression der Ventilfedern infolge Ventilhub bewirken hierbei einen einfachen Ölkreislauf und sichern die Schmierung der Komponenten. An der Ober- und Unterseite des Zylinders sind Öffnungen zum Befüllen bzw. Ablassen des Schmieröls angebracht.

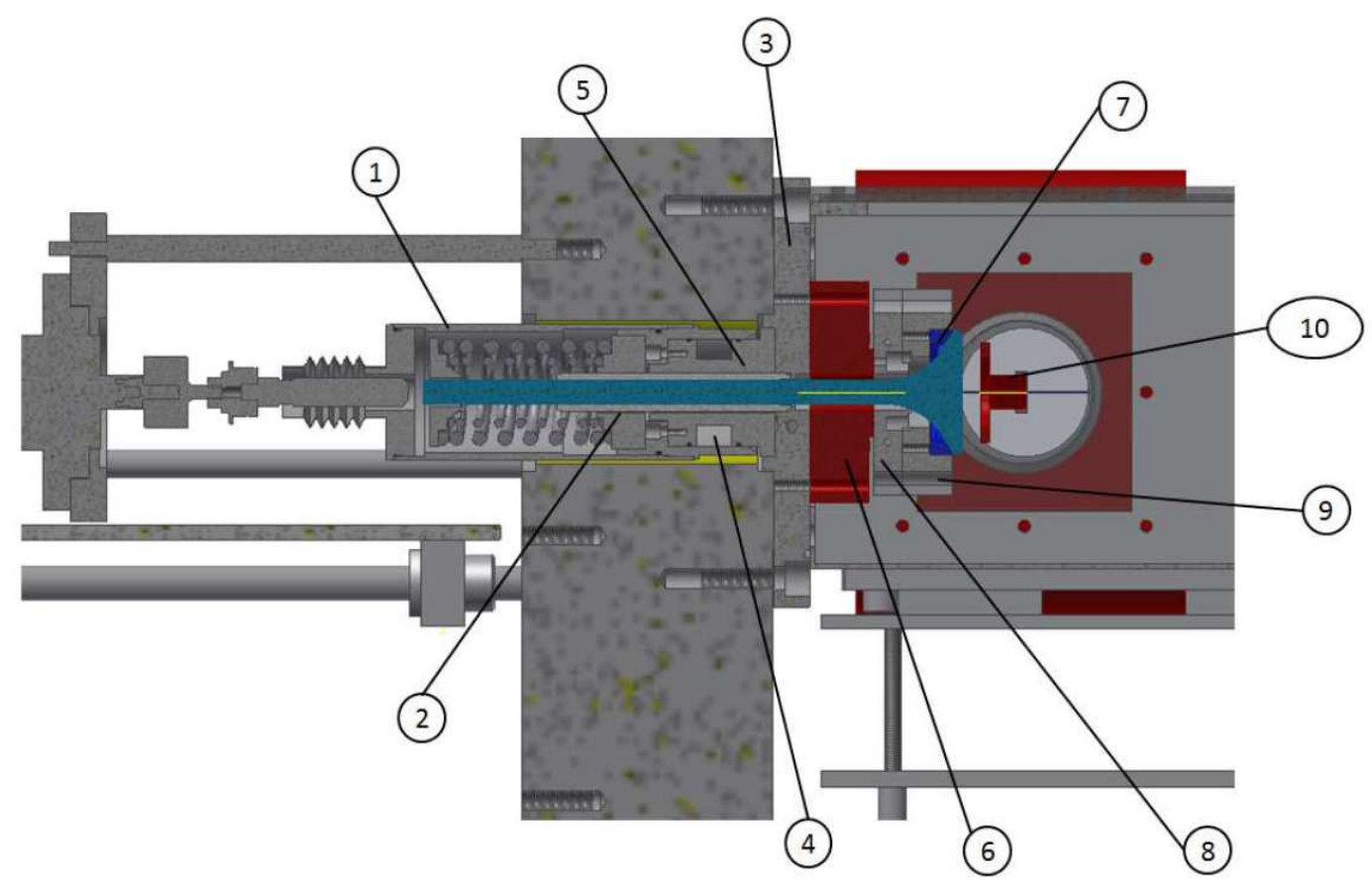

\section{Abbildung 3.14: CAD-Modell von Ölschmierung und Kühlung}

Aus [138] ist bekannt, dass Fluchtungsfehler in Bezug auf Koaxialität und Winkligkeit zwischen VS und SR zu mechanischem Verschleiß führen, daher kommt der Kühlung eine wichtige Aufgabe zu, um kühlungsbedingte Verzüge in der Ventilführung zu vermeiden. Neben der Ölkühlung verfügt der Prüfaufbau daher über zwei Wasserkreisläufe zur Kühlung von SR (7) und Ventilführung (2). Die Detailaufahme in Abbildung 3.15 veranschaulicht die drei Kühlkreisläufe aus Abbildung 3.14. Die Wasserkühlung der Ventilführung erfolgt über innenliegende Bohrungen des aus Edelstahl gefertigten Hauptflanschs (3), über welche das Kühlmedium zu den Kühlkanälen (4) strömen kann. Neben der Kühlung dient der Hauptflansch zur Positionierung eines Hohlzylinders (5), in welchen die Ventilführung eingepresst wird. Bei der Fertigung von Hauptflansch und Hohlzylinder sind die Form- und Lagetoleranzen Koaxialität und Planparallelität kritisch. Der im Prüfaufbau integrierte Kraftaufnehmer (6) dient zur Messung der Aufschlagskraft während des Ventilschließens. Da das Funktionsprinzip des verwendeten Kraftaufnehmers auf dem elektrischen Widerstand beruht, wird dieser über einen zusätzlichen Kühlflansch (8) gekühlt. Analog dem Hauptflansch besitzt der Kühlflansch innenliegende Bohrungen, die vom Kühlmedium durchströmt werden und somit konstante Temperaturen am Kraftaufnehmer gewährleisten. Für den Versuchsbetrieb ist festzuhalten, 
dass der Kraftaufnehmer während der Dauerversuche zur Vermeidung von Sensorschäden durch einen Dummy ersetzt wird. Der SR wird wie im realen Zylinderkopf in eine Tasche des Sitzringhalters (9) eingepresst und am Kühlflansch angeschraubt. Zur Erleichterung der Demontage dienen Löcher an der Halterrückseite zum Auspressen. Eine konzentrische Positionierung des Induktors (10) an der Tellerplanseite wird über eine gasdichte Öffnung in der Klimakammer realisiert (vgl. Abbildung 3.13 (b)).

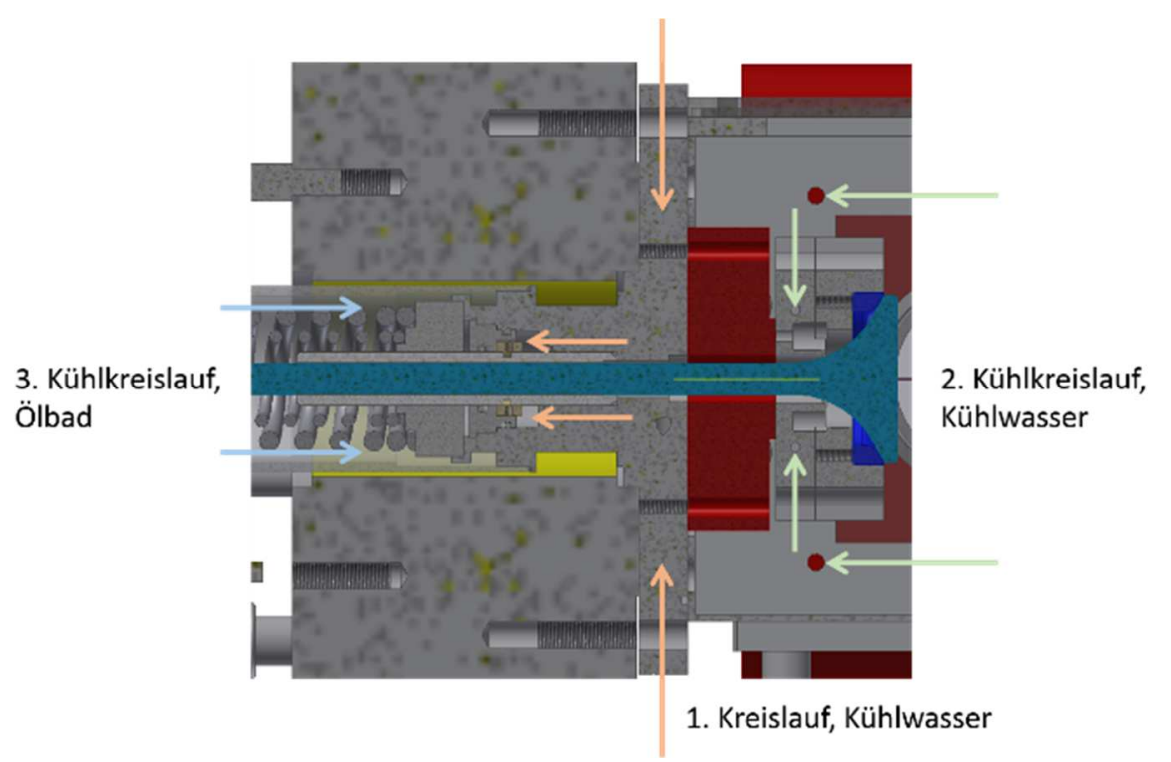

Abbildung 3.15: Detailaufnahme der Kühlkreisläufe aus Abbildung 3.14

\subsubsection{Vorversuche zum Kühlsystem}

Für die Überprüfung des Kühlsystems wurden Vorversuche zur Temperaturmessung durchgeführt. Zu diesem Zweck wurden Thermoelemente an VS und SR appliziert, wobei die VS zwei (je 1x Tellerdurchmesser und neben der Sitzfläche) und der SR vier Elemente (je 2x am Außen- und Innendurchmesser gegenüberliegend) erhielt. Zusätzlich kam ein Pyrometer an der Tellerplanfläche zur Anwendung, um für spätere Versuchsreihen ohne Applikation der Thermoelemente Vergleichswerte zu erhalten. Abbildung 3.16 (a) veranschaulicht den Versuchsaufbau zur validierten Temperaturmessung. Nach erfolgreicher Verkabelung und Installation der Regelung des Induktionsschwingkreises wurde die VS mittels der Software LabView kontrolliert auf eine Temperatur von $500{ }^{\circ} \mathrm{C}$ erwärmt. In Abbildung 3.17 sind die gemessenen Temperaturverläufe für VS und SR über die Zeit dargestellt. Die Temperaturerfassung mittels Pyrometers (schwarze Kurve) lag nach Lackieren der Planfläche mit schwarzem und hitzebeständigem Lack in einem ähnlichen Bereich wie die der beiden Thermoelemente. 


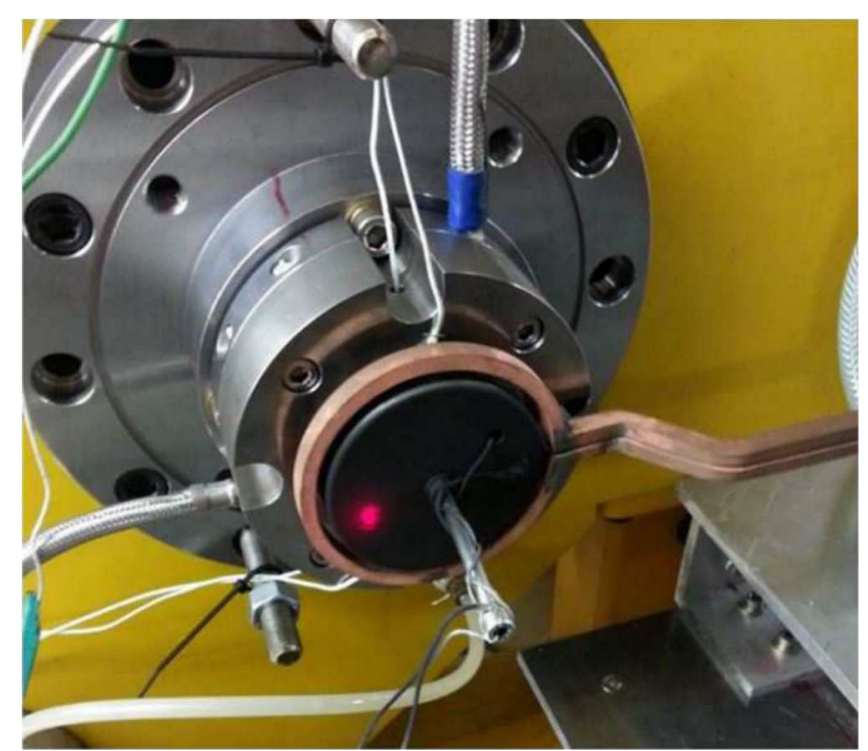

(a)

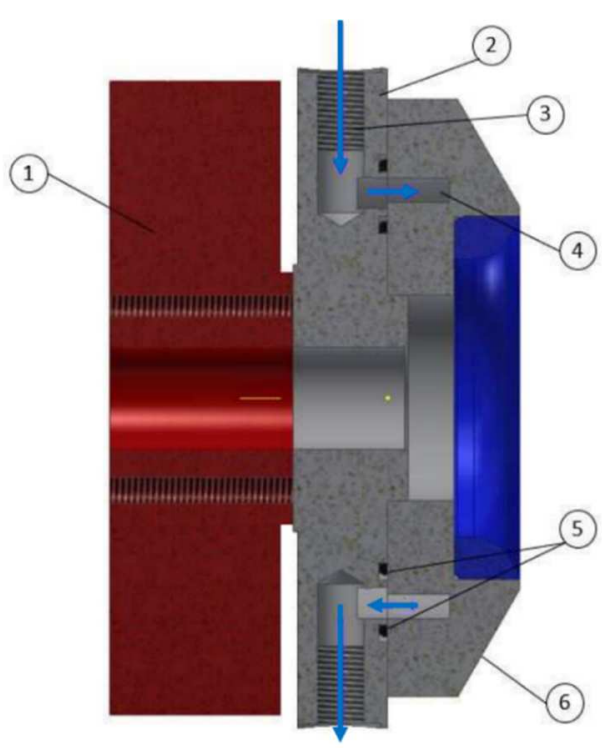

(b)

\section{Abbildung 3.16: Versuchsaufbau zur validierten Temperaturmessung (a) und überarbeitetes Kühlsystem (b)}

Im Temperaturvergleich von VS und SR ist nach einer Zeit von $1500 \mathrm{~s} z \mathrm{u}$ beobachten, dass bei einer Ventiltemperatur von ca. $450^{\circ} \mathrm{C}$ an der Tellerplanfläche (rote Kurve) der SR eine Temperatur von ca. $125^{\circ} \mathrm{C}$ (magenta Kurve) bei maximaler Kühlleistung erreicht. Das Offset von ca. $25 \mathrm{~K}$ an der Messposition „oben, außen“ (rote Kurve) vom SR erklärt sich aus der Einkopplung des Schwingkreises an dieser Stelle. Im Ergebnis war festzustellen, dass das Kühlsystem die vorgegebene Soll-Temperatur Tsoll, SR von $90^{\circ} \mathrm{C}$ bei maximaler Kühlleistung nicht gewährleisten konnte.

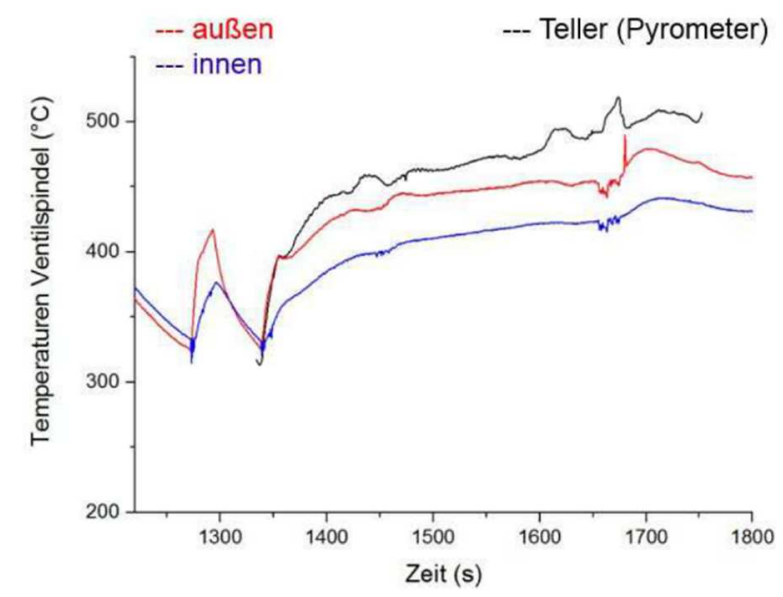

(a)

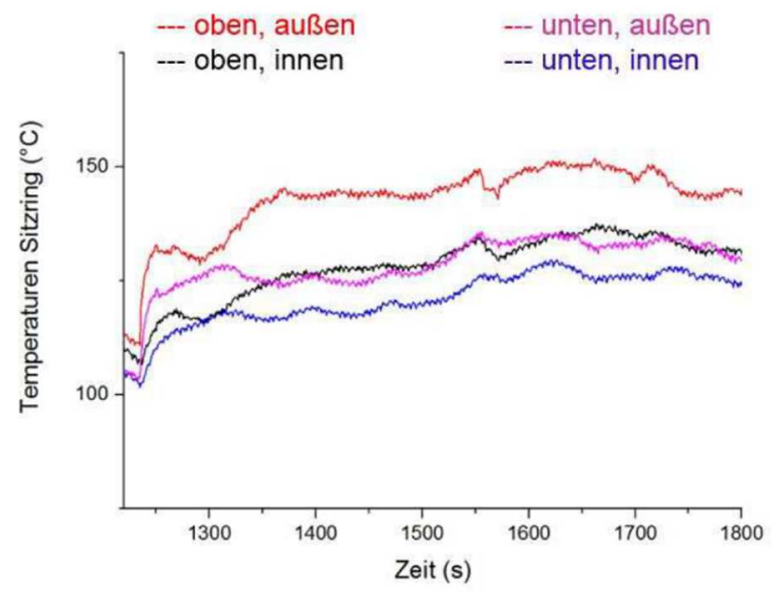

(b)

\section{Abbildung 3.17: Validierte Temperaturverläufe an VS (a) und SR (b)}

In Abbildung 3.16 (b) ist die Einbausituation nach Überarbeitung des Kühlsystem dargestellt. Hierfür wurde der Abstand zwischen Kraftsensor (1) und Kühlflansch (2) hinsichtlich einer besseren Zugänglichkeit sowie einer leichteren Applikation der Thermoelemente vergrößert. Zur Erhöhung der Kühlleistung wurden die Gewindebohrugen (3) des Kühlflanschs auf zwei 
größere G1/4"-Bohrungen erweitert. Sitzringhalter (6) und Kühlflansch erhielten zusätzliche KühInuten (4), wodurch der Sitzringhalter direkt gekühlt werden kann. Zwei temperaturbeständige O-Ringe (5) Typ Viton gewährleisten die Abdichtung. Einem zusätzlichen Wärmeeintrag am Sitzringhalter infolge Einkopplung des Induktionsschwingkreises wird mit einer vergrößerten Fase (6) entgegengewirkt.

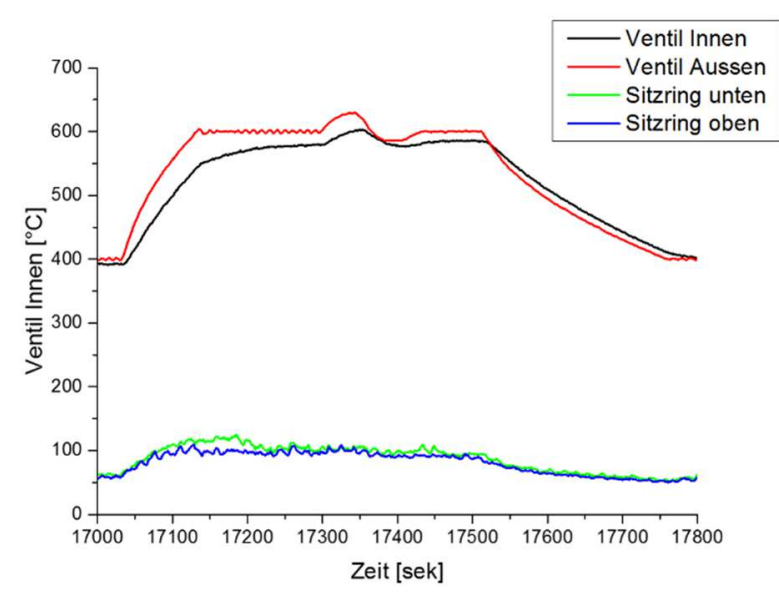

(a)

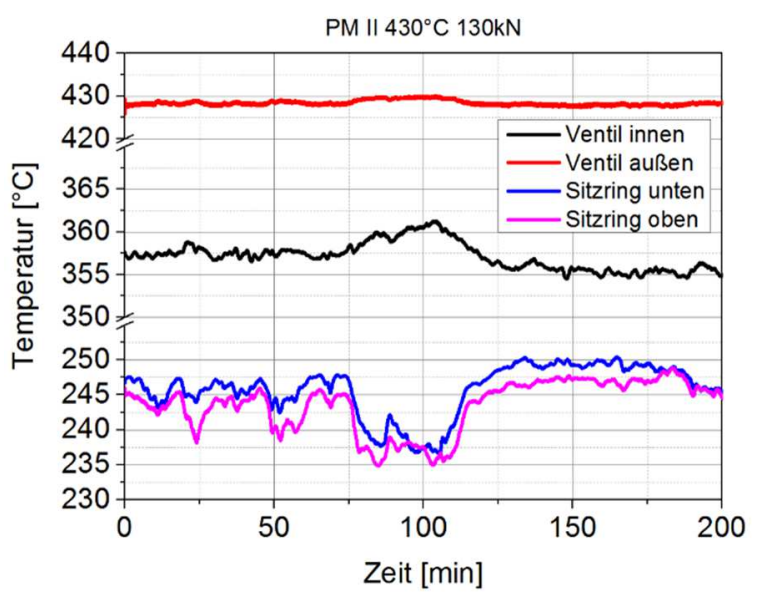

(b)

Abbildung 3.18: Validierte Temperaturmessungen; (a) Auszug aus einer 5-stündigen Prüfmessung bei verschiedenen Temperaturen bis $600^{\circ} \mathrm{C}$ im Prüfmodus I unter $0,6 \mathrm{~m} / \mathrm{s}$, (b) Auszug aus einem Vorversuch im Prüfmodus II unter $130 \mathrm{kN}$ bei $430{ }^{\circ} \mathrm{C}$

In Abbildung 3.18 (a) ist ein Auszug aus einer 5-stündigen Prüfmessung des überarbeiteten Kühlsystems dargestellt. Bei der erneuten Validierung konnte eine hinreichende Kühlleistung für Versuchstemperaturen bis zu $600{ }^{\circ} \mathrm{C}$ unter $0,6 \mathrm{~m} / \mathrm{s}$ Schließgeschwindigkeit nachgewiesen werden. Im Prüfmodus II wirken infolge größerer Prüfkräfte höhere Flächenpressungen und längeren Kontaktzeiten zwischen VS und SR, wodurch der Wärmeübergang beeinflusst wird. Die erhöhte Wärmezufuhr im Vergleich zu Modus I erfordert eine höhere Kühlleistung. Abbildung 3.18 (b) veranschaulicht den Auszug einer Temperaturmessung unter $130 \mathrm{kN}$ bei $430{ }^{\circ} \mathrm{C}$. Das gemessene Delta zwischen VS und SR beträgt abhängig von der Messposition ca. 180 bis $195 \mathrm{~K}$.

\subsubsection{Prüfmodus II - Simulation des Verbrennungsdrucks}

Für die Konstruktion der Versuchsanordnung von Prüfmodus II war ebenso wie für Prüfmodus I die Verwendung von realen Komponenten die Vorgabe (vgl. Kapitel 3.1). In Erweiterung zum Prüfmodus I wird der Prüfaufbau von Modus II am linken und rechten Maschinenjoch montiert. Abbildung 3.19 veranschaulicht Prüfmodus II als CAD-Modell im Halbschnitt. Eine Zusammenbauzeichnung mit Stückliste findet sich im Anhang (Abbildung 8.3). Der Prüfaufbau besteht aus den fünf Hauptbaugruppen servohydraulisch-mechanischer Ventildrehmechanismus (1), Ventilsystem (2), gasdichter Klimakammer (3), Druckstempel (4) und Prüfrahmen (5). Zur Erzeugung der druckäquivalenten Prüfkraft drückt der Stempel (4) auf die Tellerplanfläche. Damit eine gleichmäßige Kraftübertragung erreicht und mögliche 
Biegemomente infolge Querkräfte vermieden werden, ist eine koaxiale Ausrichtung von Ventil- und Stempelachse entscheidend. Für diesen Zweck wird ein Koppelelement zwischen Stempel und Ventilteller zur Kraftübertragung verwendet. Die Einbausituation ist in der Detailaufnahme Abbildung 3.20 dargestellt. Zur besseren Visualisierung wurde ein virtueller Spalt, hervorgehoben durch ein rotes Rechteck, zwischen den sphärisch gewölbten Adapterstücken (E) und (F) mit einem kontaktseitigen Radius von $500 \mathrm{~mm}$ eingefügt. Die so erzeugte Gelenkpfanne zwischen Hydraulikstempel (G) und Koppelelement (D) soll einen möglichen axialen Versatz ausgleichen. Als Werkstoff für das Koppelelement wurde ein nichtleitender Werkstoff gewählt. Dadurch wird eine Entkopplung des Stempels vom Induktor und somit eine ausschließliche Erwärmung des Ventiltellers erreicht. Am Anfang kam Feinglimmer, ein Elektroisolierstoff aus silikonharzimprägniertem Glimmerpapier, später aufgrund der kurzen Standzeiten Siliziumnitrid zum Einsatz.

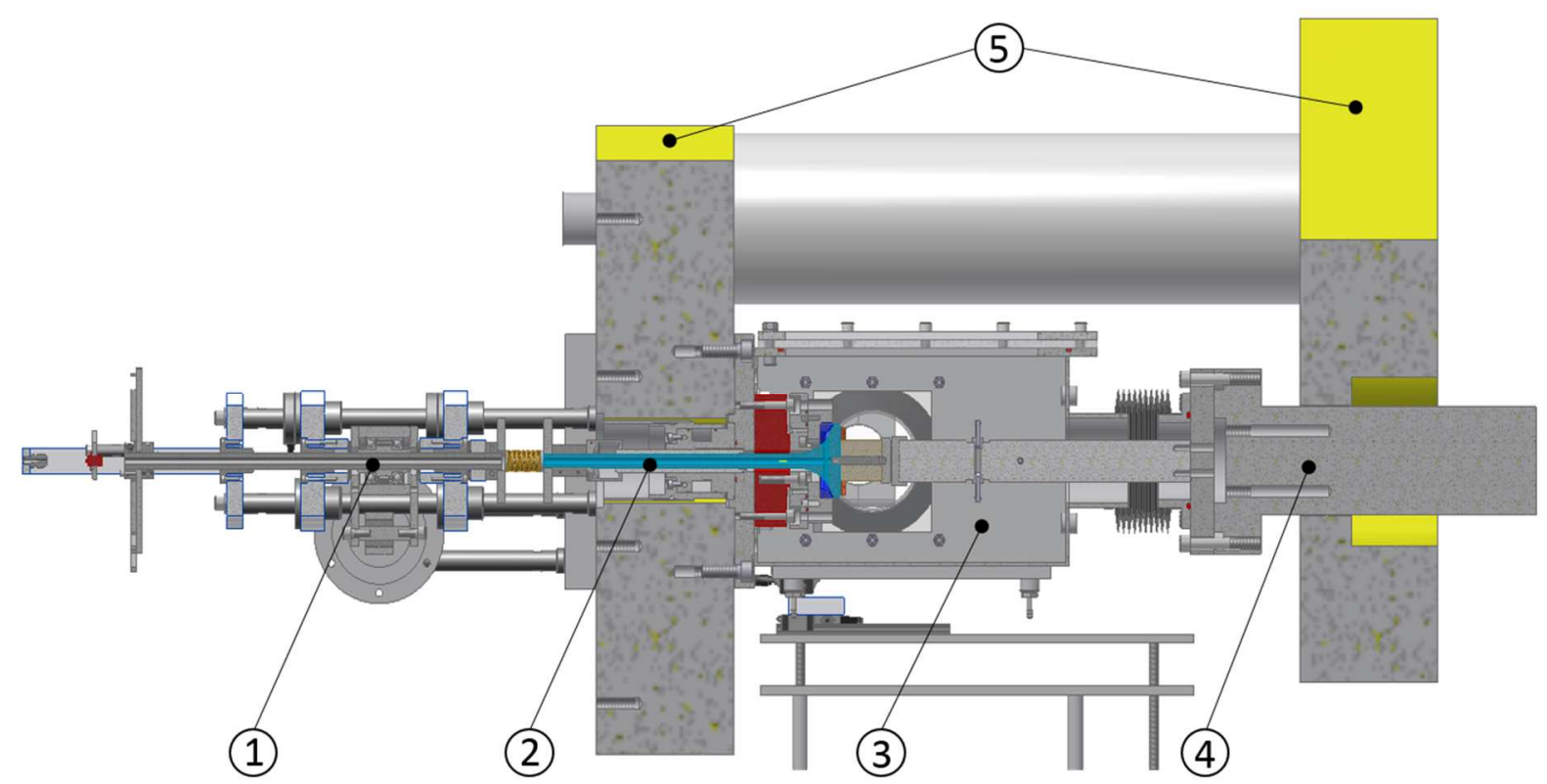

\section{Abbildung 3.19: CAD-Modell von Prüfmodus II im Halbschnitt}

Zur querkraftfreien Lasteinleitung kommt ein Passungsbolzen (C) mit einer spielfreien Übergangspassung zur Anwendung. Als positiver Nebeneffekt vereinfachte sich dadurch der Probenwechsel. Über die in der VS (B) erkennbaren Bohrungen werden die Signalleitungen der oberflächennah applizierten Thermoelemente des Typs $\mathrm{K}$ geführt. Wie im Kapitel 3.1.2 erwähnt, wurde während der Konzeptphase deutlich, dass der benötigte Ventilhub von $25 \mathrm{~mm}$ in Kombination mit geforderten Prüffrequenz von 8,3 Hz und Prüfkraft bis $150 \mathrm{kN}$ nicht umsetzbar ist. In der Folge konnte die originale Ventildrehvorrichtung nicht verwendet werden, da diese ventilhubabhängig funktioniert. An der Stelle sei erwähnt, dass das Funktionsprinzip der Drehvorrichtung eine bestimmte Ventilfederkraft bedingt. Die Ventilfederkräfte steigen linear mit dem Ventilhub und drücken mit Erreichen einer bestimmten Ventilfederkraft die Tellerfeder in der Drehvorrichtung herunter, wodurch die 
Kugeln im Gehäuse der Drehvorrichtung konzentrisch um die Ventilachse rotieren und eine Ventilrotation bewirken.

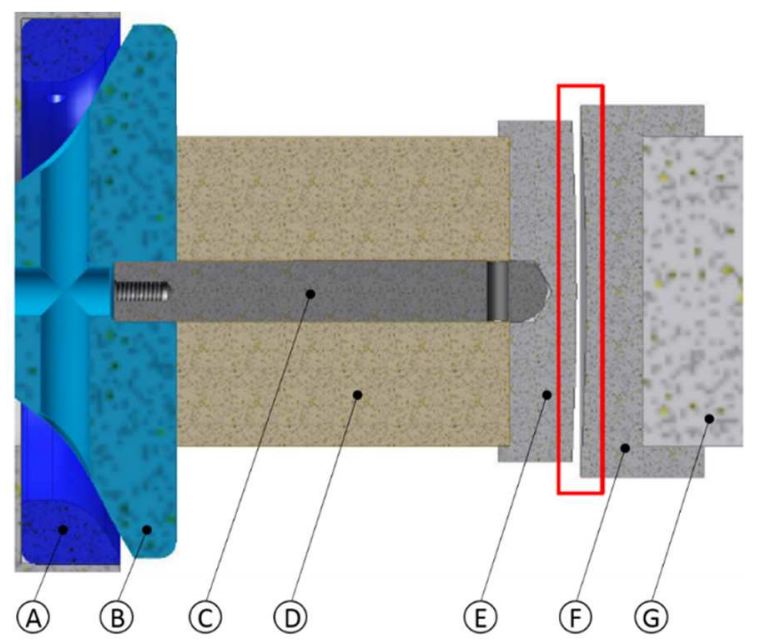

\section{Abbildung 3.20: Detailaufnahme aus Abbildung 3.19; Einbausituation von VS/Druckstempel im Halbschnitt als CAD-Modell}

Für die technische Umsetzung der Ventilrotation wurde daher ein zusätzliche Baugruppe erforderlich. Der servohydraulisch-mechanische Drehmechanismus ist in Abbildung 3.21 dargestellt. Die Wahl eines zweiten Hydraulikzylinders als Aktuator schafft einerseits hinsichtlich der Steuerung die Möglichkeit, die Ventilrotation in Abhängigkeit vom Stempelzylinder phasenverschoben genau zu regeln, andererseits konnte der Zylinder aus Prüfmodus I verwendet werden. Die technische Herausforderung ergibt sich aus dem phasenverschobenen Betrieb beider Hydraulikzylinder.

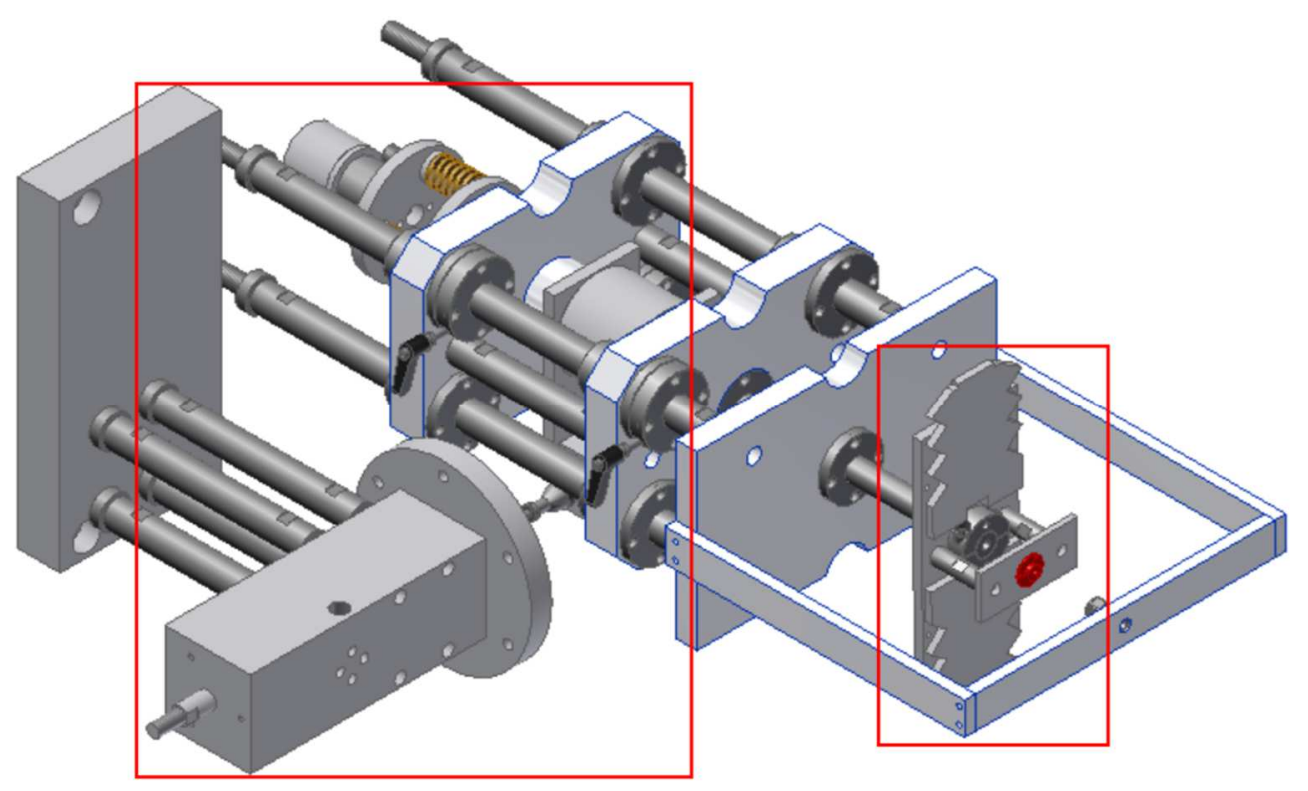

Abbildung 3.21: Hydraulisch-mechanischer Drehmechanismus als CAD-Modell 
Zum Zeitpunkt der Entlastung des Hauptzylinders muss die Belastung des Nebenzylinders erfolgen. Die Baugruppe Ventildrehmechanismus wurde kompakt konstruiert. Sie kann von der Baugruppe Ventilsystem z.B. für Wartungs- oder Ausrichtungszwecke getrennt und um $100 \mathrm{~mm}$ linear verschoben werden. Somit ist bei Entkopplung der beiden Baugruppen eine ausreichende Zugänglichkeit zu den Komponenten sichergestellt. Die durch das kleine rote Rechteck hervorgehobene Unterbaugruppe in Abbildung 3.21 ist für die Entkopplung der kabelgebundenen Thermoelemente wichtig. Die in einer Hohlwelle rotierende Signalleitungen werden über einen Schleifringüberträger entkoppelt. Zuvor werden die Signale über Messumformer auf 0 bis $10 \mathrm{~V}$ verstärkt, um mögliche Übertragungsverluste des Schleifringüberträgers zu reduzieren. Abbildung 3.22 veranschaulicht eine reduzierte Darstellung, der in Abbildung 3.21 durch ein großes rotes Rechteck hervorgehobenen Unterbaugruppe. Der sinusförmig angesteuerte Nebenzylinder (I) ist $90^{\circ}$ zur Ventilachse angeordnet und bewegt auf Druck über den Gelenkkopf (II) den Freilauf (III), wobei die Kugelgelenkverbindung zum Ausgleich der Kreisbewegung des Freilaufs dient. Über eine Welle treibt der Freilauf auf Druck das Federpaket (V) an. Der Freilauf schafft konstruktiv die Möglichkeit, die VS während des Ventilöffnens zu drehen. Über die Einstellung des Freilaufs wird die Ventilrotation eingestellt. Positiver Nebeneffekt des Drehmechanismus ist, dass die Ventilrotation gezielt verändert und als Einflussfaktor auf den Ventilverschleiß zu einem späteren Zeitpunkt untersucht werden könnte. In der Ventilphase Schließen erfolgt keine Rotation. Bei Zugbelastung erfolgt ein Loslösen der einzelnen Klemmkörper und der Freilauf dreht sich ohne Rotation der Welle zurück. Am Ende der Welle ist die Scheibe (IV) befestigt, die mit dem Federpaket (V) verbunden ist.

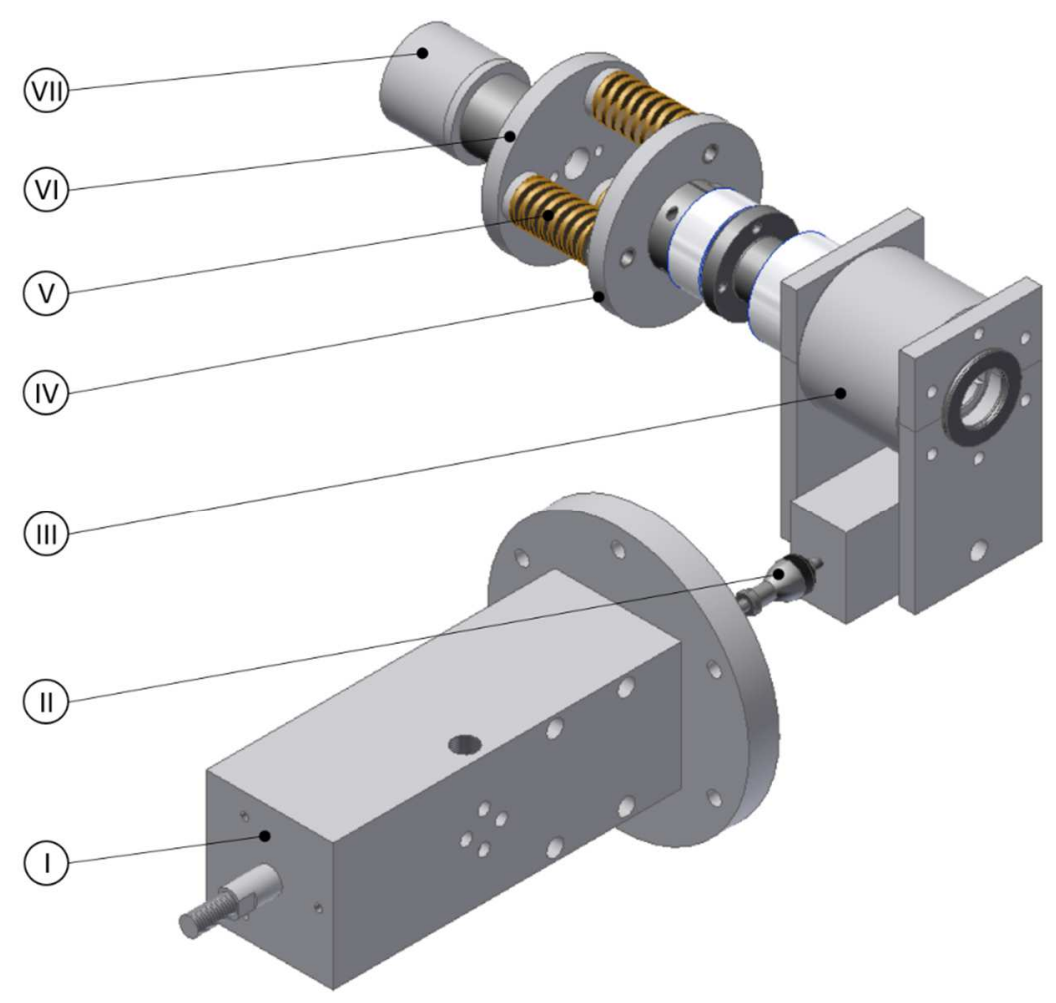

\section{Abbildung 3.22: Reduzierte Darstellung der Baugruppe Ventildrehmechanismus als CAD-Modell}


Um eine Entkopplung der axialen Belastung zu erreichen, wurde die Verbindung über Führungsstifte innerhalb der Federn realisiert. Dadurch ist es möglich, dass die rechte Scheibe (IV) losgelöst von der linken Scheibe (VI) nur eine rotatorische Bewegung ausübt. Die Notwendigkeit der Federn ergibt sich aus der Bedingung, dass die VS in kontinuierlichem Kontakt mit dem Hauptzylinder bleiben soll. Bei der Konfiguration muss der Kolben des Nebenzylinders einen Hub von $12,2 \mathrm{~mm}$ zurücklegen, um einen Drehwinkel von $8^{\circ}$ je Ventilhub zu erreichen. Dieser Winkel entspricht der Rotation der originalen Drehvorrichtung. Nach Formel (3.2) ergibt sich die druckäquivalente Prüfkraft $F_{P}$ als Produkt aus Zünddruck $p_{Z}$ und Tellerplanfläche $A_{T}$ (Formel (3.1).

$$
\begin{gathered}
A_{T}=\frac{\pi}{4} \times d_{T}^{2} \\
F_{P}=p_{Z} \times A_{T}
\end{gathered}
$$

Für einen Zünddruck $p_{Z}$ von 180 bar ergibt sich bei dem in dieser Arbeit verwendeten Ventiltellerdurchmesser $d_{T}$ von $86 \mathrm{~mm}$ eine Prüfkraft $F_{P}$ von 104,56 kN. Vorversuche mit einer Ventilschließgeschwindigkeit von $0,1 \mathrm{~m} / \mathrm{s}$ verdeutlichten keinen Einfluss des Parameters Schließgeschwindigkeit. Aus diesem Grund werden alle Versuche im Prüfmodus II mit dieser Geschwindigkeit gefahren. Das verwendete Hydraulikaggregat ermöglicht bei der geforderten Prüffrequenz von $8,3 \mathrm{~Hz}$ eine Prüfkraft bis $150 \mathrm{kN}$. Dies entspricht einem simulierten Zünddruck $p_{Z}$ von rund 260 bar bei einem Ventiltellerdurchmesser von $86 \mathrm{~mm}$.

\subsubsection{Messtechnik und Peripherie}

Für die Messung der Aufschlagskraft in Prüfmodus I wurde ein Kraftaufnehmer des Typs DR der Firma GTM Testing and Metrology GmbH mit einem Messbereich von $\pm 50 \mathrm{kN}$ verwendet, hingegen kam für Prüfmodus II ein Aufnehmer des Typs $K$ derselben Firma mit einem Messbereich von $\pm 250 \mathrm{kN}$ zur Anwendung. In Abbildung 3.23 ist die Aufschlagskraft als Funktion der Ventilschließgeschwindigkeit am Anfang und nach 4.000 Betriebsstunden dargestellt. Eine signifikante Änderung der Aufschlagskraft ist nicht erkennbar. Messungenauigkeiten aufgrund verschleißender Komponenten wie z. B. Ventilfedern oder Ventilführung sind somit vernachlässigbar klein. Zur Programmierung der Versuchsdurchführung wurde die Software WaveMatrix ${ }^{\mathrm{TM}}$ der Firma INSTRON GmbH für dynamische Versuche verwendet. Diese eignet sich für zyklische Prüfmethoden und ermöglicht eine grafische und matrixbasierte Vorschau des Prüfablaufs. Ferner regelt WaveMatrix ${ }^{\mathrm{TM}}$ den Hydraulikzylinder mittels des induktiven Sensors LVDT (Linear Variable Differential Transformer) zur Wegmessung. Alle Messdaten wurden mit der Software LabVIEW der Firma National Instruments Corporation aufgezeichnet. LabVIEW dient nicht nur zur Regelung der Ventiltemperatur, sondern überwacht Temperaturen an kritischen Stellen des Versuchsaufbaus und löst z. B. bei Ausfall der Kühlung eine Sicherheitskette aus. 


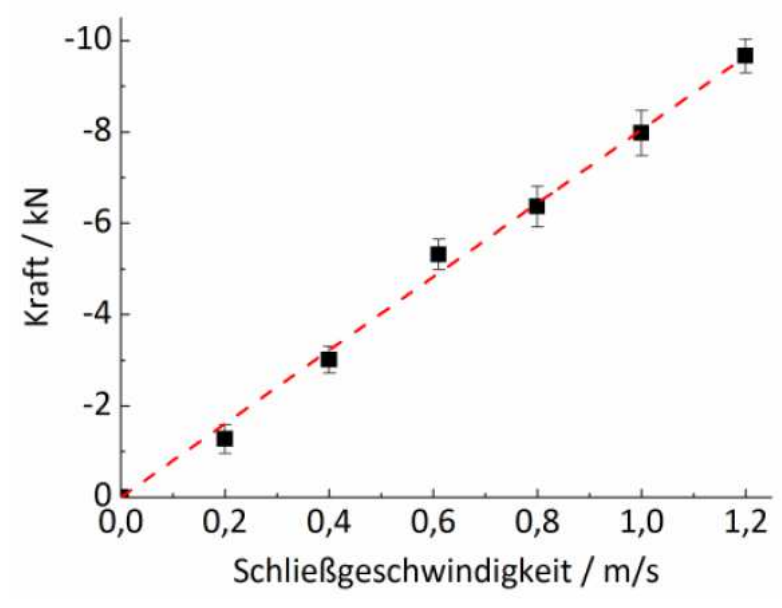

(a)

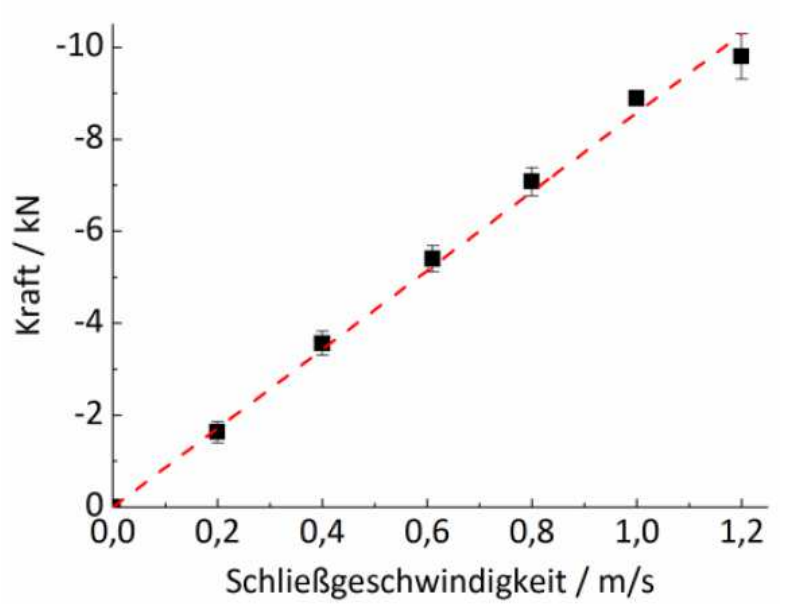

(b)

Abbildung 3.23: Messung der Aufschlagskraft als Funktion der Schließgeschwindigkeit im Prüfmodus I am Anfang (a) und nach ca. 4.000 Betriebsstunden (b)

Im Fall einer Störung schaltet LabVIEW ein Relais durch, dass die Gaszufuhr unterbricht und das Hydraulikaggregat sowie die Induktionsanlage ausschaltet.

Bei Verwendung einer Nockenwelle definiert die Nockenform den Verlauf der Ventilbewegung. Charakteristisch für Nockenkurven sind die positiven und negativen Beschleunigungsrampen zu Beginn und am Ende. Kritisch für den Ventilverschleiß ist die negative Rampe, mit welcher die Ventilschließgeschwindigkeit bestimmt wird. Diese kann vereinfacht als Dreiecksfunktion angenähert werden. Daher wurde aus dem Funktionsgenerator von WaveMatrix ${ }^{\mathrm{TM}}$ eine anwendungsähnliche Dreiecksfunktion als Steuerkurve für den Ventilhub verwendet. Abbildung 3.24 veranschaulicht die Position des weggesteuerten Hydraulikzylinders im Prüfmodus I über die Zeit von drei Messzyklen.

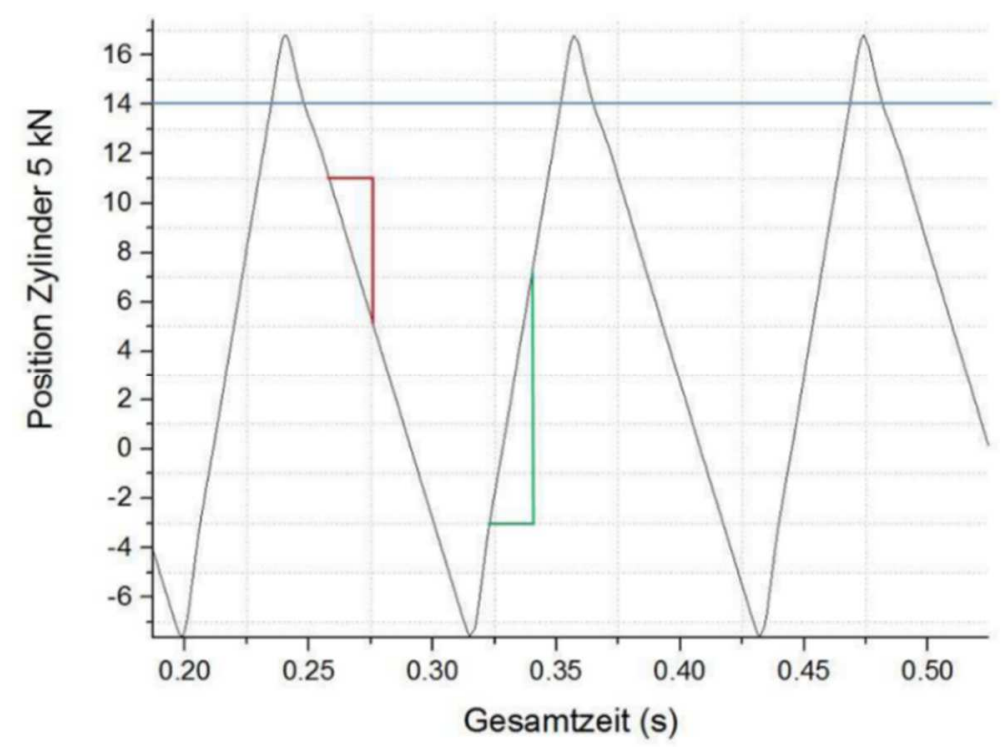

Abbildung 3.24: Ausschnitt einer Wegmessung, Position des weggesteuerten Hydraulikzylinders im Prüfmodus I über die Zeit von drei Messzyklen 
Die blaue Linie bei $14 \mathrm{~mm}$ markiert jene Position des Hydraulikzylinders, bei der Kraftschluss zwischen Stößel und VS herrscht. Mit dem Erreichen dieser Zylinderposition werden die Kontaktflächen im weiteren Verlauf entkoppelt. Die eingezeichneten Steigungsdreiecke beschreiben die Ventilgeschwindigkeiten. Das Öffnen erfolgt mit $0,3 \mathrm{~m} / \mathrm{s}$ (rotes Dreieck), das Schließen mit 0,6 m/s (grünes Dreieck) bei einer Prüffrequenz von 8,3 Hz. Der Ventilhub beträgt $22 \mathrm{~mm}$. Mit dieser Prüfmethode wurden alle Versuche im Prüfmodus I durchgeführt. In Abbildung 3.25 ist eine Weg- und Kraftmessung des Druckstempels im Prüfmodus II über einen Zyklus dargestellt. Die dreiecksförmig eingeleitete Prüfkraft beträgt $130 \mathrm{kN}$ und der sinusförmige Stempelhub ca. $5 \mathrm{~mm}$.

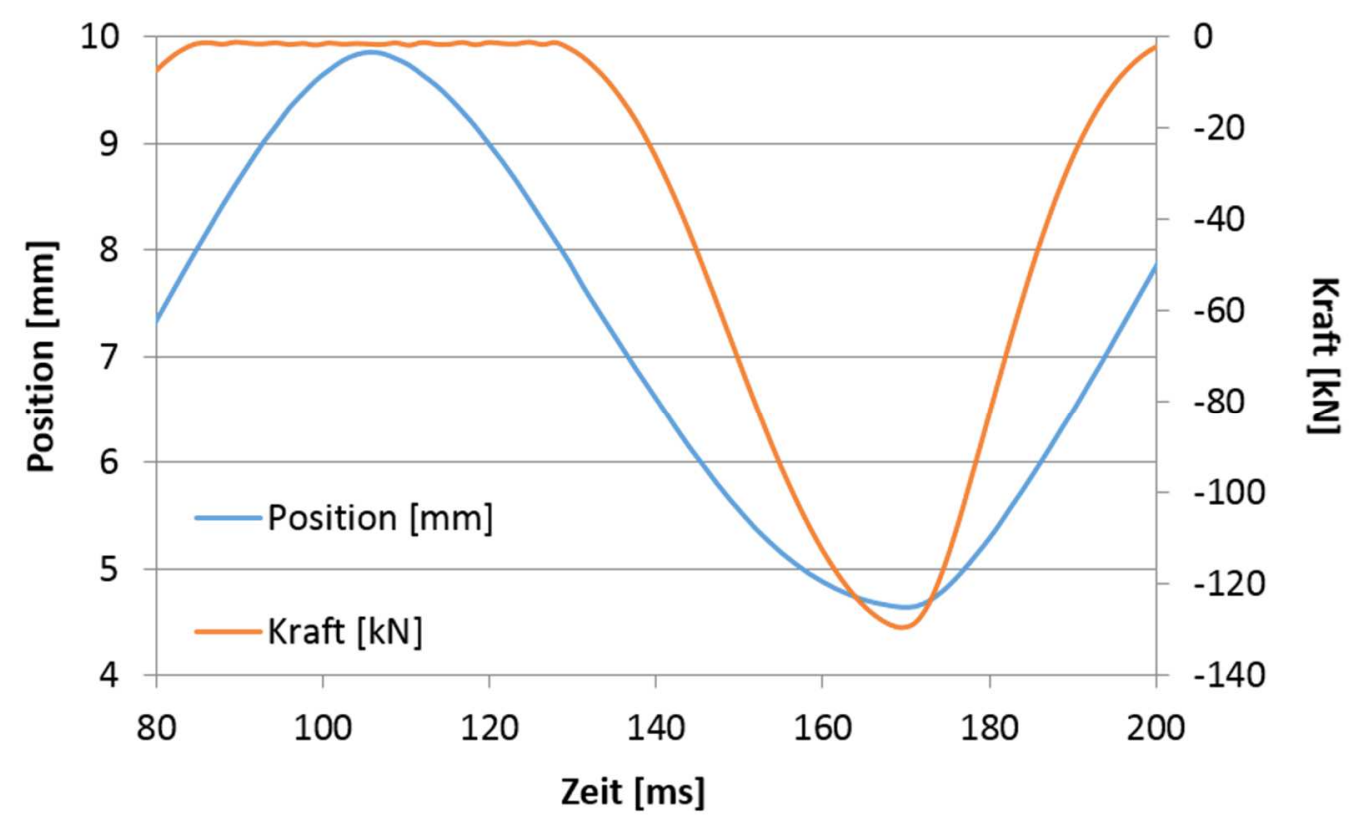

\section{Abbildung 3.25: Weg- und Kraftmessung des Druckzylinders im Prüfmodus II über einen Zyklus}

\subsubsection{Ausrichtung des Prüfaufbaus}

Ein wichtiger Aspekt des Prüfaubaus ist die Koaxialität von VS und SR, um untypische Verschleißerscheinungsformen zu vermeiden. Anderenfalls kann es zu hohem Verschleiß kommen, wie ihn Malatesta et al. in [138] beschrieben haben. Daneben könnten unzulässige Biegemomente infolge Querkraft im Ventilschaft entstehen.

Alle Komponenten wurden mit engen Toleranzen gefertigt. Trotzdem bildet sich beim Zusammenbau mehrerer Bauteile zu einer Baugruppe eine Maßkette, die die Funktionsfähigkeit nach [186] beeinflusst. Die sich ergebende Schließtoleranz kann das Funktionieren eines Zusammenbaus blockieren. Neben den tolerierten geometrischen Maßen sind für die Funktionsfähigkeit Form- und Lagetoleranzen von Bedeutung. Um an der Stelle dem Thema Toleranzverknüpfungen zu begegnen, wurde auf eine Toleranzanalyse und Toleranzsynthese verzichtet. Stattdessen kam eine Einbauhilfe zur Sicherstellung der Koaxialität zur Anwendung. Grundelement für die koaxiale Ausrichtung des Prüfaufbaus ist 
der Hauptflansch am linken Maschinenjoch. Beim Aufbau des Prüfrahmens ist die Durchgangsbohrung im linken Joch als Passung gefertigt worden. Diese dient als Zentrierung des Hauptflansches. Der Hauptflansch widerum wurde mit einer Sacklochbohrung von 25 mm gefertigt. Für die Ausrichtung von Kraftaufnehmer, Kühlflansch und Sitzringhalter wird zu diesem Zweck eine Zentrierwelle als Einbauhilfe in diese Bohrung gesteckt. Da der Sitzringhalter einen größeren Durchmesser als die $25 \mathrm{~mm}$ aufweist, wird für dessen Ausrichtung ein Zentrierring auf die Zentrierwelle geschoben. Abbildung 3.26 veranschaulicht die Montagesituation während des Ausrichtens.

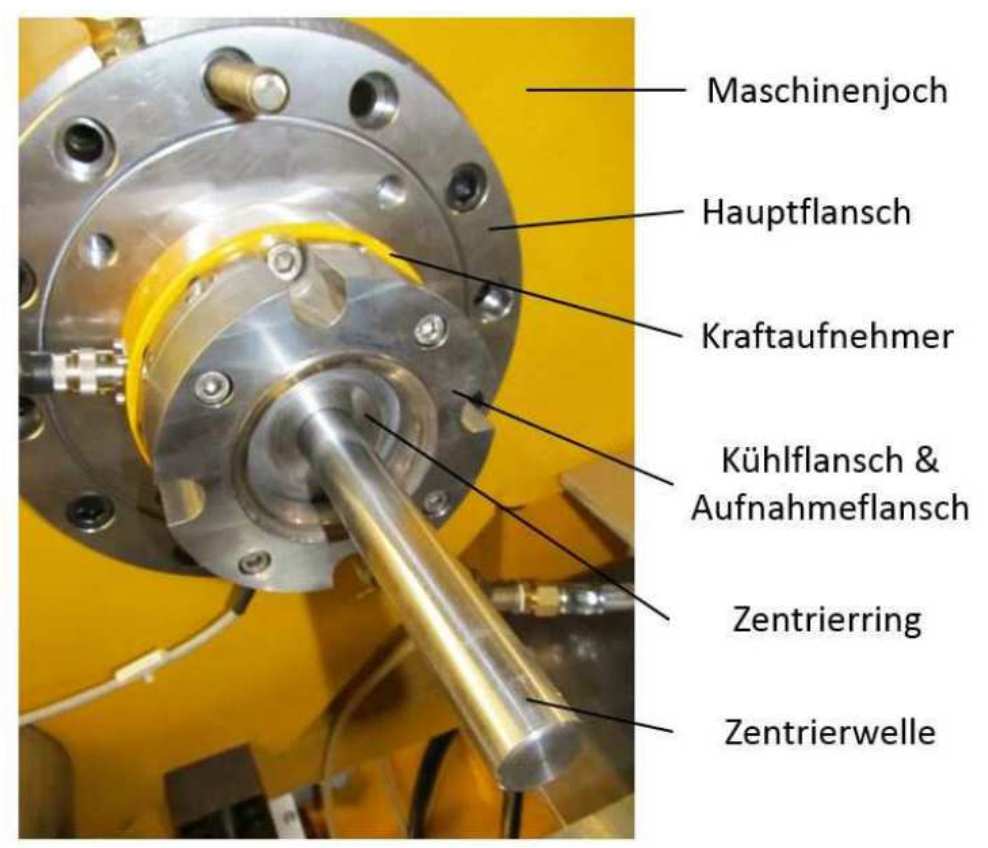

\section{Abbildung 3.26: Ausrichten von Kraftaufnehmer, Kühlflansch und Sitzringhalter mittels Zentrierwelle und Zentrierring}

Neben der Lagetoleranz Koaxialität ist die Winkligkeit funktionsrelevant. Diese wird von der Planparallelität bestimmt. Daher wurden alle Kontaktflächen der Flansche und Halter planparallel geschliffen. Eine schräge Fläche hätte denselben Effekt wie ein koaxialer Versatz.

\subsection{Versuchsablaufplan}

In Abbildung 3.27 ist der 3-stufige Ablaufplan eines typischen $100 \mathrm{~h}$-Versuchs mit den wesentlichen Arbeitsschritten dargestellt. Beginnend mit der Versuchsvorbereitung werden an den neuen Prüfteilen VS und SR Tiefenprofilmessungen durchgeführt. Anschließend werden die Komponenten eingebaut und die Thermoelemente appliziert. Nach dem Einstellen der Versuchsparameter und dem Festlegen der Grenzwerte für die Sicherheitskette startet der Versuch. Die intervallmäßigen Messungen der Verschleißtiefenprofile an der VS erfolgen nach 25, 50, 75 und $100 \mathrm{~h}$. Nach $100 \mathrm{~h}$ wird der Versuch beendet. Die Nachbereitung startet mit der Demontage von VS und SR. Die Tiefenprofile von VS und SR werden ermittelt. Anschließend werden repräsentative Segmente aus den Sitzbereichen beider Komponenten herauserodiert und makro- wie mikroskopisch charakterisiert. 


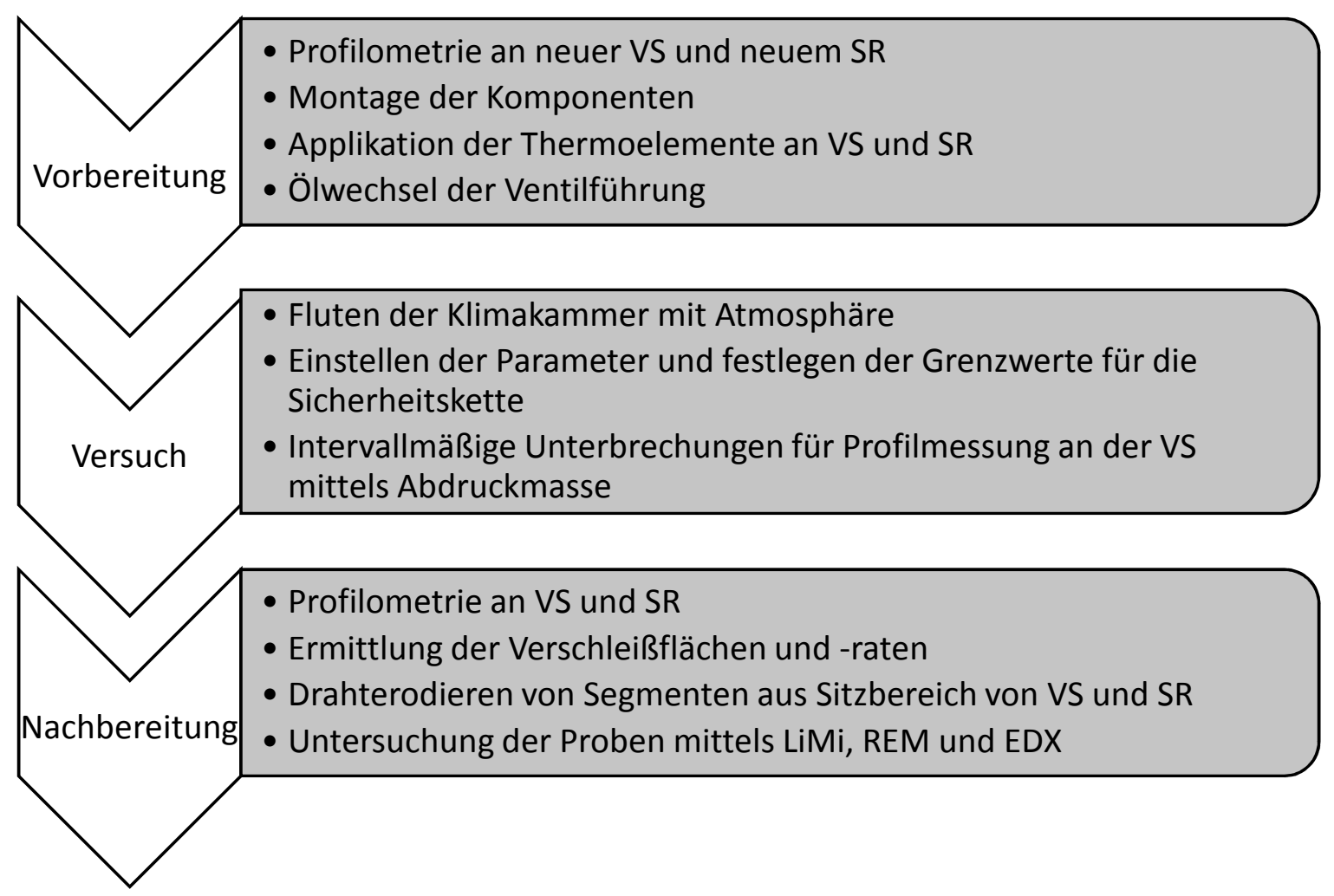

Abbildung 3.27: 3-stufiger Ablaufplan eines typischen $100 \mathrm{~h}-$ Versuchs

\subsection{Validierung des Ventilverschleißprüfstands}

In diesem Kapitel soll die Validierung des VVPs vorgestellt werden. Nach den beiden mechanischen Kriterien Ventilrotation und Kühlsystem sollen die tribologischen Kenngrößen Verschleißerscheinungsform und Verschleißrate betrachtet werden. Die Nachbildung der eigenschaftsbestimmenden Verschleißmechannismen mittels experimenteller Simulation bestimmt die Maschinenfähigkeit des neuentwickelten VVPs.

\subsubsection{Ventilrotation}

Da das Prinzip zur Validierung der Ventilrotation für beide Prüfmodi gleich ist, wird im Folgenden nur die Validierung für den Prüfmodus Ventilschließen vorgestellt. Der integrierte Kraftaufnehmer ermöglicht die Messung von Druck- und Zugkräften in Achsrichtung sowie des Drehmoments um dieselbige. Mit der im Prüfaufbau verwendeten originalen Ventildrehvorrichtung kommt es im realen Motorbetrieb zu einer Rotation während des Ventilöffnens. Dies wurde in Vorversuchen gegengeprüft. Die VS wird vom SR entkoppelt und beginnt zu rotieren. Mit dem Erreichen des maximalen Hubs endet die Rotation. Für den Fall einer Drehmomentmessung müsste es zu einer Ventilrotation während des Ventilschließens und somit zu einem übertragenen Drehmoment kommen. Dies würde eine Fehlfunktion der Prüfanordnung bedeuten. Bei den beobachteten Verschleißuntersuchungen im Kapitel 2.3.1 konnten keine Hinweise auf eine Ventilrotation im Kontakt mit dem SR gefunden werden. Abbildung 3.28 veranschaulicht einen repräsentativen Drehmomentverlauf über vier 
Messzyklen für den verwendeten $5 \mathrm{kN}$-Hydraulikzylinder. Erkennbar ist ein deutliches Rauschen aufgrund der Sensorkalibrierung in Verbindung mit der hohen Dynamik des Systems. Die Größe des gemessenen Drehmoments beträgt $\pm 1 \mathrm{Nm}$ und ist damit vernachlässigbar klein. In Bezug auf die Ventilrotation ist der Prüfaufbau validiert.

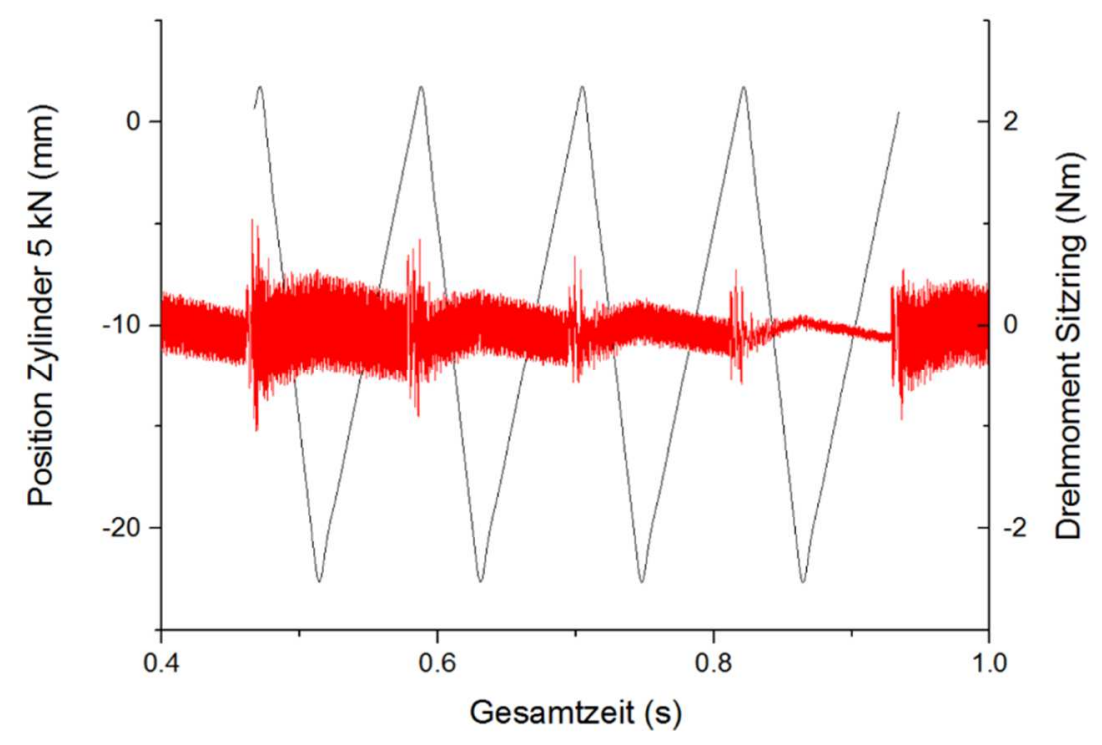

Abbildung 3.28: Validierte Drehmomentmessung zur Ventilrotation über vier Messzyklen im Prüfmodus I

\subsubsection{Kühlsystem}

Für die Validierung des Kühlsystems sind zwei Aspekte entscheidend. Zum einen gilt es die Versuchstemperaturen über die Versuchsdauer konstant zu halten, zum anderen müssen mit dem eingesetzten Kühlsystem die geplanten Temperaturen zuverlässig realisiert werden können. Der Kühlung kommt somit im Hinblick auf eine kontrollierte Parametervariation eine entscheidende Bedeutung zu. In den zu einem späteren Zeitpunkt geplanten Versuchsreihen ist beabsichtigt, die Temperatur in einem typischen Bereich von 330 bis $430{ }^{\circ} \mathrm{C}$ für EVSn in Großmotoren nach [187] zu variieren, wobei der ESR meist um das Delta von ca. 200 bis $300 \mathrm{~K}$ nach [188] kälter ist. Da die Variationen in den späteren Versuchsreihen zum Ventilschließen $0,2,0,6$ und 1,0 m/s und zum Verbrennungsdruck 140, 180 und 220 bar betragen sollen, ergab sich für die Validierung des Kühlsystems eine 3-stufige Parametervariation. Die Validierung bestand somit aus sechs Vorversuchen. Abbildung 3.29 veranschaulicht repräsentative Messverläufe aus den Vorversuchen zu beiden Prüfmodi I und II. An je zwei Messpositionen der VS wurden in den Bereichen des SADs und des SIDs Thermoelemente appliziert, wobei die Temperatur am SAD die Regelgröße für die induktive Heizung war. Am SR wurden die Temperaturen an zwei gegenüberliegenden Positionen (unten und oben) im Bereich des SADs gemessen, wobei die Temperatur an der Stelle oben die Regelgröße für die drei Kühlkreisläufe war. In Tabelle 3.4 sind die Minimal-und Maximalwerte für die einzelnen Messpositionen wiedergegeben. 
Betrachtet man die Temperaturen der Regelgröße am SAD der VS, so betragen die maximalen Deltas $11 \mathrm{~K}$ und $5 \mathrm{~K}$ für Ventilschließen bzw. Verbrennungsdruck. Vergleicht man die minimalen SR-Temperaturen mit den maximalen Werten am SAD der VS, so ergeben sich maximale Deltas von $321 \mathrm{~K}$ und $198 \mathrm{~K}$ für Ventilschließen bzw. Verbrennungsdruck.

Tabelle 3.4: Minimal- und Maximalwerte an den Messpositionen von VS und SR

\begin{tabular}{|c|c|c|c|c|c|c|c|}
\hline \multirow[b]{2}{*}{ Nr. } & \multirow[b]{2}{*}{ Vorversuch } & \multirow[b]{2}{*}{ Einheit } & & \multicolumn{2}{|c|}{ VS } & \multicolumn{2}{|c|}{ SR } \\
\hline & & & & SID & SAD & unten & oben \\
\hline \multirow[t]{2}{*}{1} & $330{ }^{\circ} \mathrm{C}-0,2 \mathrm{~m} / \mathrm{s}$ & ${ }^{\circ} \mathrm{C}$ & Min. & 303 & 319 & 120 & 145 \\
\hline & & & Max. & 320 & 330 & 153 & 168 \\
\hline \multirow[t]{2}{*}{2} & $380{ }^{\circ} \mathrm{C}-0,6 \mathrm{~m} / \mathrm{s}$ & ${ }^{\circ} \mathrm{C}$ & Min. & 361 & 379 & 136 & 131 \\
\hline & & & Max. & 370 & 381 & 155 & 150 \\
\hline \multirow[t]{2}{*}{3} & $430{ }^{\circ} \mathrm{C}-1,0 \mathrm{~m} / \mathrm{s}$ & ${ }^{\circ} \mathrm{C}$ & Min. & 381 & 428 & 117 & 134 \\
\hline & & & Max. & 419 & 438 & 138 & 148 \\
\hline \multirow[t]{2}{*}{4} & $330{ }^{\circ} \mathrm{C}-140$ bar & ${ }^{\circ} \mathrm{C}$ & Min. & 293 & 330 & 153 & 164 \\
\hline & & & Max. & 302 & 335 & 176 & 176 \\
\hline \multirow[t]{2}{*}{5} & $380^{\circ} \mathrm{C}-180$ bar & ${ }^{\circ} \mathrm{C}$ & Min. & 323 & 379 & 203 & 232 \\
\hline & & & Max. & 333 & 381 & 214 & 241 \\
\hline \multirow[t]{2}{*}{6} & $430{ }^{\circ} \mathrm{C}-220$ bar & ${ }^{\circ} \mathrm{C}$ & Min. & 354 & 426 & 236 & 232 \\
\hline & & & Max. & 361 & 430 & 250 & 249 \\
\hline
\end{tabular}

Als Gemeinsamkeit beider Prüfmodi ist eine höhere Temperatur am SAD der VS im Vergleich zum SID auszumachen, wobei das Delta im Prüfmodus Verbrennungsdruck mit ca. $60 \mathrm{~K}$ erkennbar größer ist als im Modus Ventilschließen mit ca. $25 \mathrm{~K}$. Anzumerken ist, dass der Hub im Prüfmodus Ventilschließen mit 25 mm um 15 mm größer als im Modus Verbrennungsdruck ist. 


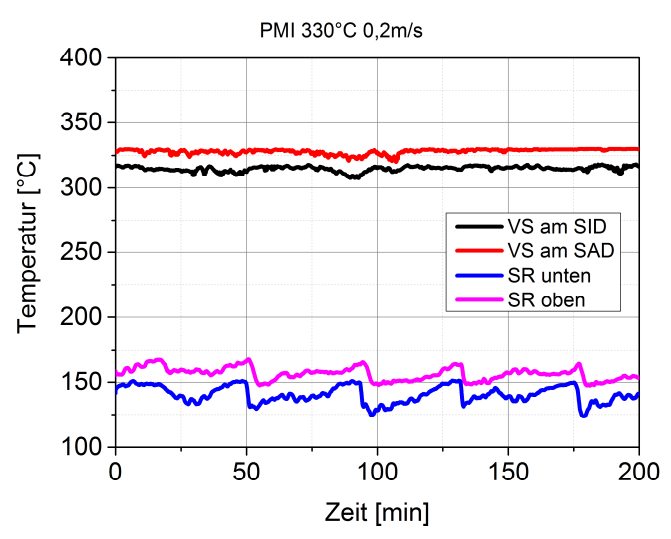

$330^{\circ} \mathrm{C}$ und $0,2 \mathrm{~m} / \mathrm{s}$

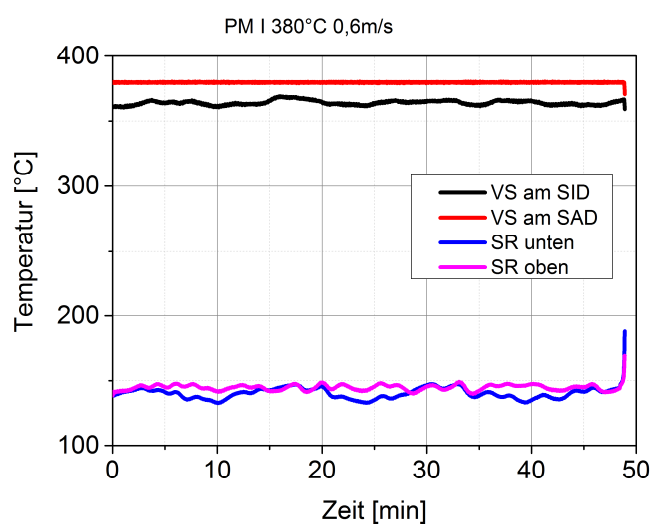

$380^{\circ} \mathrm{C}$ und $0,6 \mathrm{~m} / \mathrm{s}$

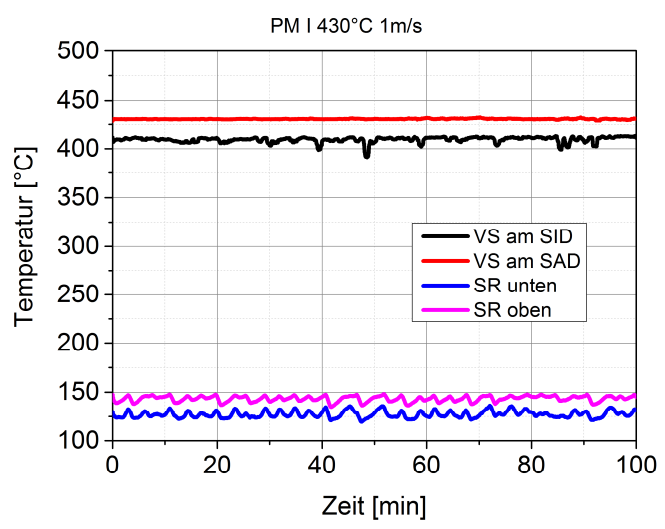

$430{ }^{\circ} \mathrm{C}$ und $1,0 \mathrm{~m} / \mathrm{s}$

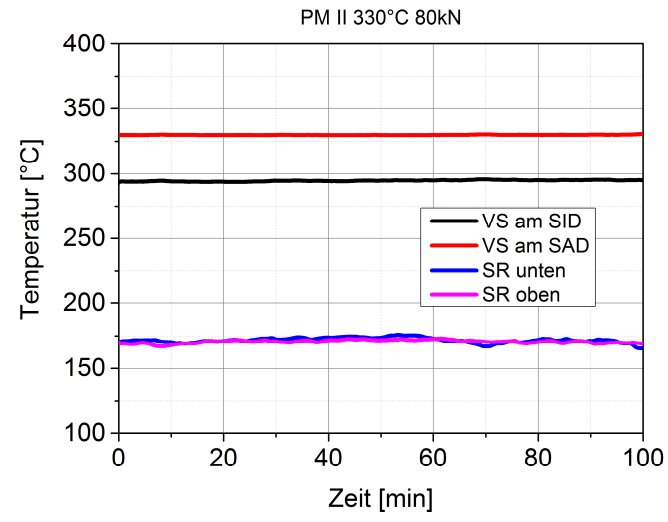

$330{ }^{\circ} \mathrm{C}$ und 140 bar

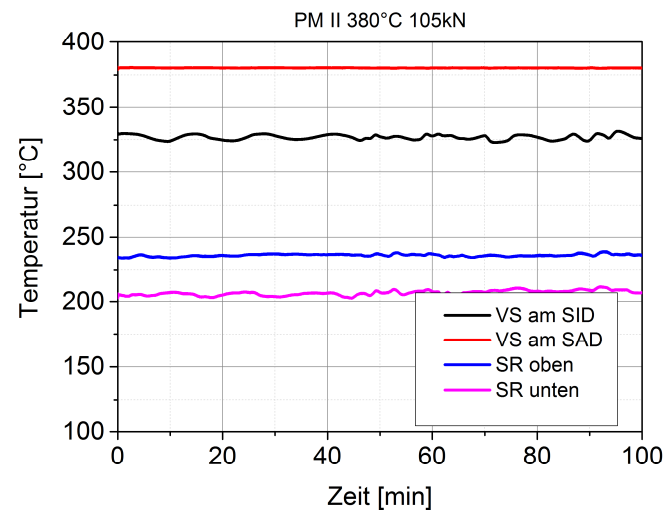

$380{ }^{\circ} \mathrm{C}$ und 180 bar

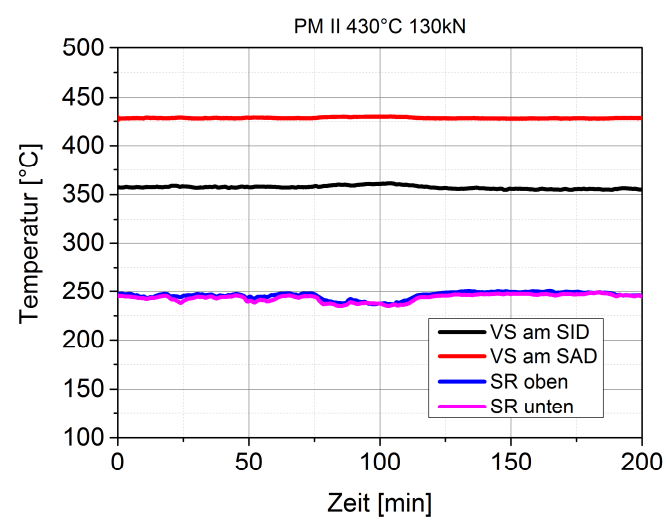

$430{ }^{\circ} \mathrm{C}$ und 220 bar

Abbildung 3.29: 3-stufig validierte Temperaturmessungen zum Kühlsystem in den beiden Prüfmodi I und II

\subsubsection{Verschleißerscheinungsformen}

Verschleißerscheinungen sind Veränderungen der Kontaktflächen eines Körpers sowie die Art und Form der entstandenen Verschleißpartikel infolge einer tribologischen Belastung [15]. Ziel einer erfolgreichen Validierung ist es somit, die für den Ventilverschleiß eigenschaftsbestimmenden Erscheinungen des Gleit- und Stoßverschleiß nachzuweisen. Für die Validierung wurden repräsentative Draufsichten und Querschliffe aus Vorversuchen zu 
den beiden Prüfmodi Ventilschließen und Verbrennungsdruck ausgewählt. Da die späteren Versuchsreihen nur bei Hochtemperatur gefahren werden sollen, ist es wichtig, die Fähigkeit temperaturinduzierte Veränderungen für den VVP zu demonstrieren. Zu diesem Zweck wurden Versuche bei Raumtemperatur und $330^{\circ} \mathrm{C}$ vergleichend untersucht. Abbildung 3.30 veranschaulicht die Erscheinungen unter $0,6 \mathrm{~m} / \mathrm{s}$ für den Prüfmodus Ventilschließen im Bereich des SIDs. Bei Raumtemperatur beträgt die Verschleißmarkenbreite ca. $3 \mathrm{~mm}$ und entspricht in etwa der halben nominellen Ventilsitzbreite (Abbildung 3.30 (a)). Hingegen erreicht sie bei $330^{\circ} \mathrm{C}$ mit $6,6 \mathrm{~mm}$ die nominelle Sitzbreite (Abbildung 3.30 (b)). In der Draufsicht sind bei Raumtemperatur noch die fertigungstypischen Schleifriefen zusammen mit einer Fältelung der Schleifspitzen zu erkennen (Abbildung 3.30 (c)). Wohingegen Schuppen und Kuppen in Verbindung mit kleinen Löchern die Draufsicht des Versuchs bei $330^{\circ} \mathrm{C}$ kennzeichnen (Abbildung 3.30 (d)). In den Querschliffen wird gleichfalls ein unterschiedliches Schädigungsbild erkennbar. Ein deutlicher Dehnungsgradient mit zerbrochenen Hartphasen ist zu beobachten (Abbildung 3.30 (f)). Daneben sind mikrometergroße Verschleißpartikel mit parallelem Rissverlauf zur Oberfläche zu sehen. Hingegen sind bei Raumtemperatur vereinzelte Risse in den Hartphasen ohne Dehnungsgradient zu erkennen (Abbildung 3.30 (e)). Ein Vergleich der Verschleißerscheinungen für den Prüfmodus Verbrennungsdruck unter 180 bar bei Raumtemperatur und $330^{\circ} \mathrm{C}$ ist in Abbildung 3.31 dargestellt. Im Kontrast zum Ventilschließen entspricht die Verschleißmarkenbreite bei Raumtemperatur bereits der nominellen Ventilsitzbreite (Abbildung $3.31(\mathrm{a})$ ). Bei $330{ }^{\circ} \mathrm{C}$ ist sie vergleichbar groß (Abbildung 3.31 (b)). In der Draufsicht bei Raumtemperatur sind noch die typischen Schleifriefen nach dem Sitzfertigschleifen im Bereich des SIDs zu sehen, wobei sich eine plastische Deformation in Form eines Übergleitens der Schleifspitzen darstellt (Abbildung 3.31 (c)). Topografisch erscheint die Verschleißspur bei $330^{\circ} \mathrm{C}$ mit augenscheinlich größeren Schuppen und Kuppen im Vergleich zum Versuch bei Raumtemperatur (Abbildung 3.31 (d)). In den Querschliffen sind keine Dehnungsgradienten in der tribologisch beanspruchten Randschicht zu erkennen. An der Oberfläche des Versuchs bei $330^{\circ} \mathrm{C}$ sind mikrometergroße Verschleißpartikel und eine ca. $1 \mu \mathrm{m}$ strukturlos erscheinende Randschicht zu beobachten (Abbildung $3.31(f)$ ).

Im Vergleich der Verschleißerscheinungsformen von Motor- und Prüfstandsteilen sind für den gleichen tribotechnischen Werkstoff Stellite ${ }^{\mathrm{TM}} 12$ dieselben Schädigungen erkennbar. Der VVP kann daher als validiert betrachtet werden. 


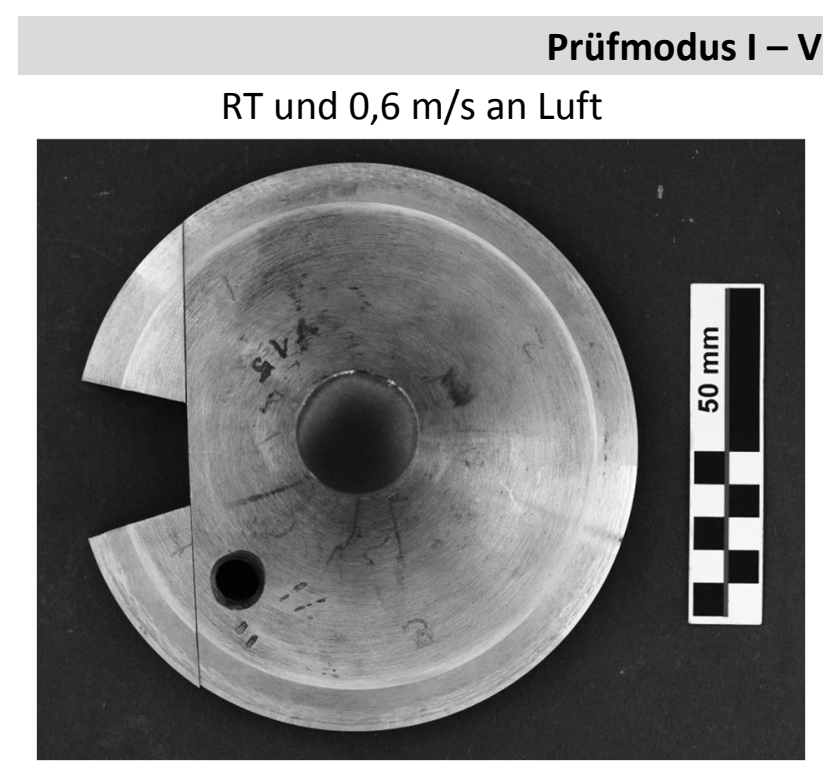

(a)

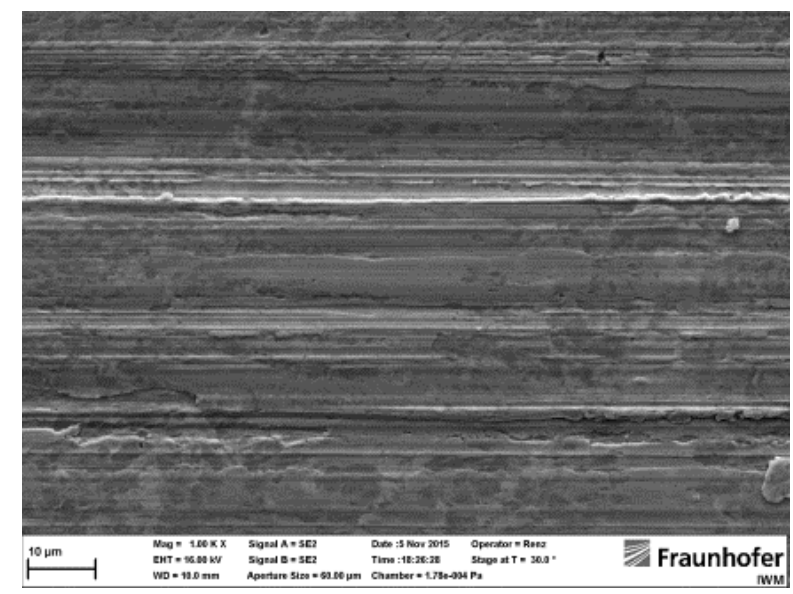

(c)

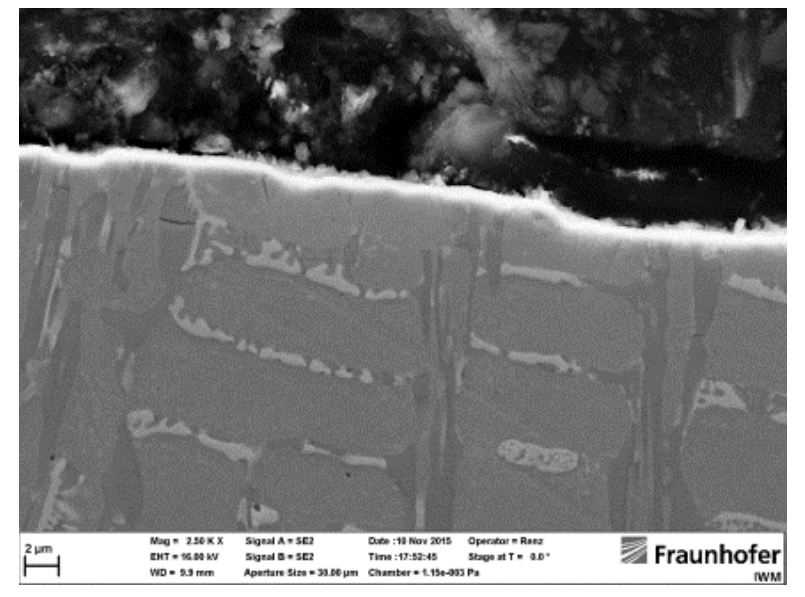

(e)

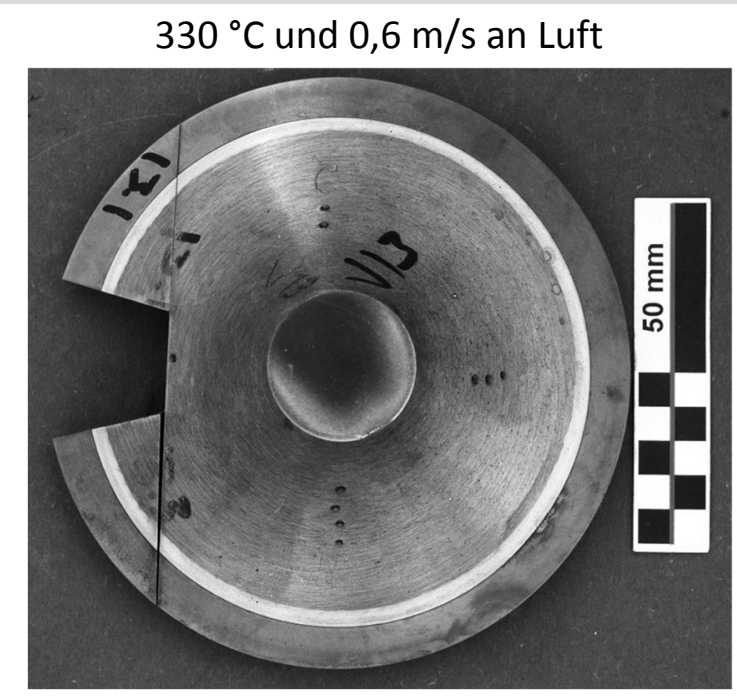

(b)

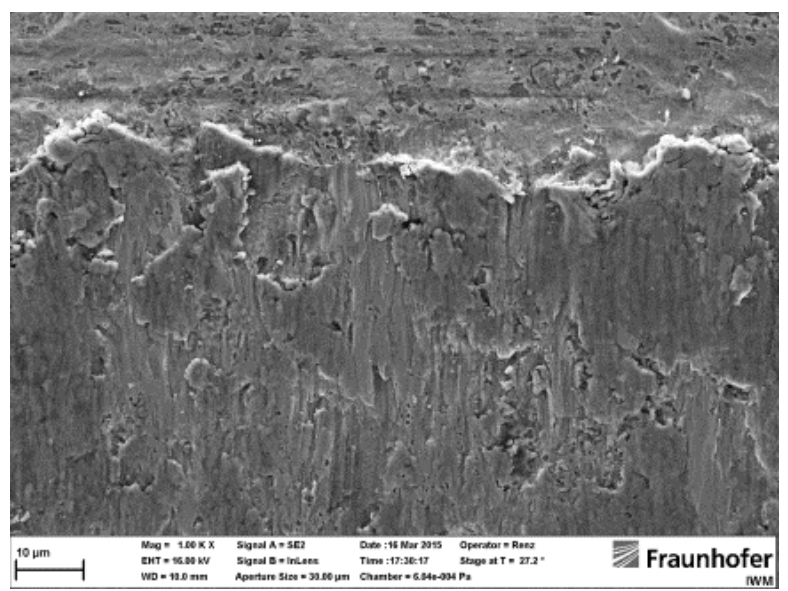

(d)

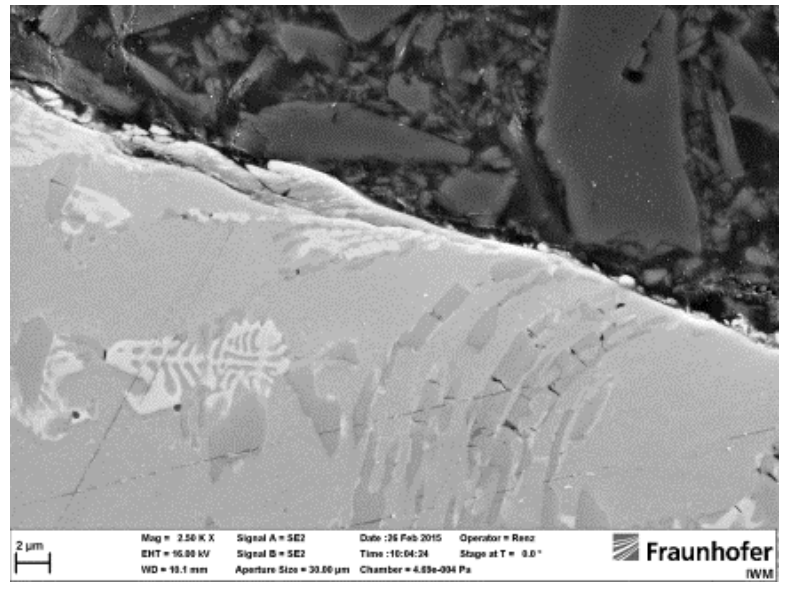

(f)

Abbildung 3.30: Vergleich der Verschleißerscheinungen unter Ventilschließen, Makroaufnahmen von den Ventiltellern (a) und (b), REM-Aufnahmen von den Draufsichten (c) und (d) sowie den zugehörigen Querschliffen (e) und (f) 


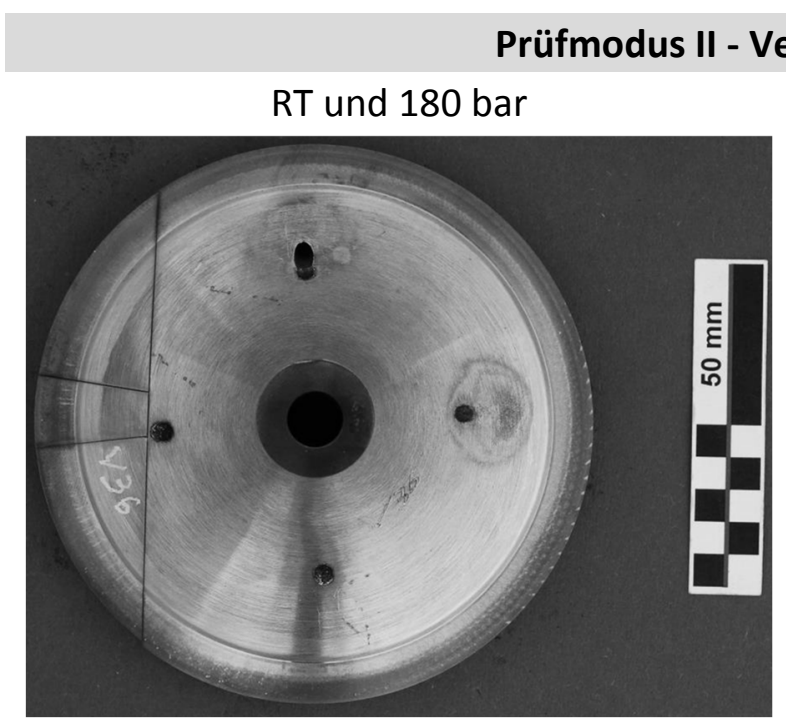

(a)

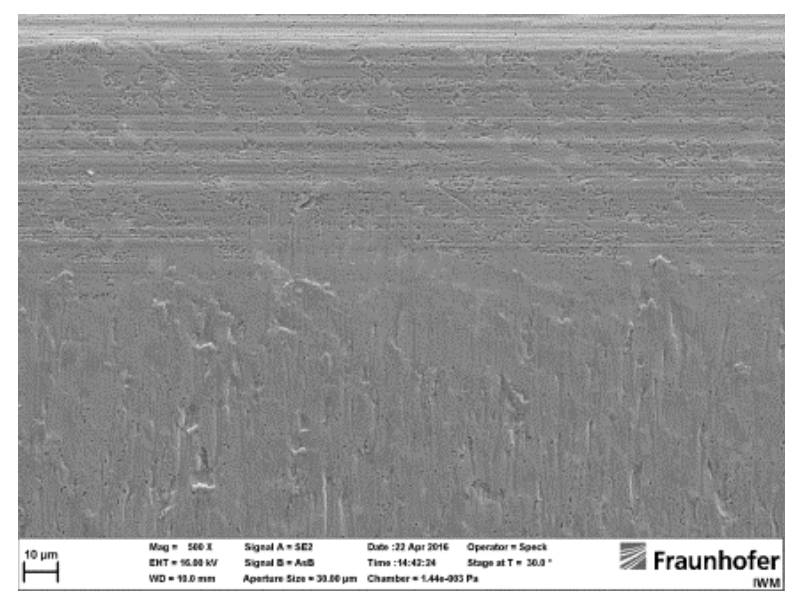

(c)

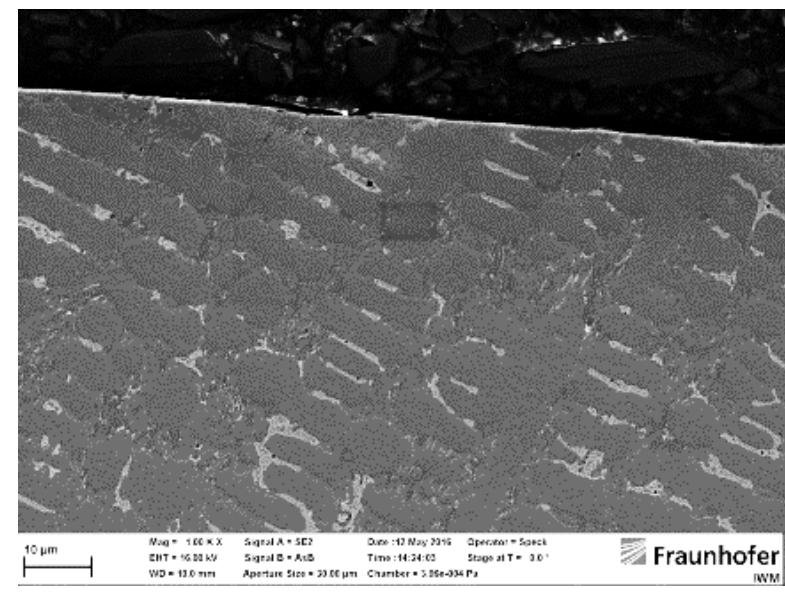

(e)

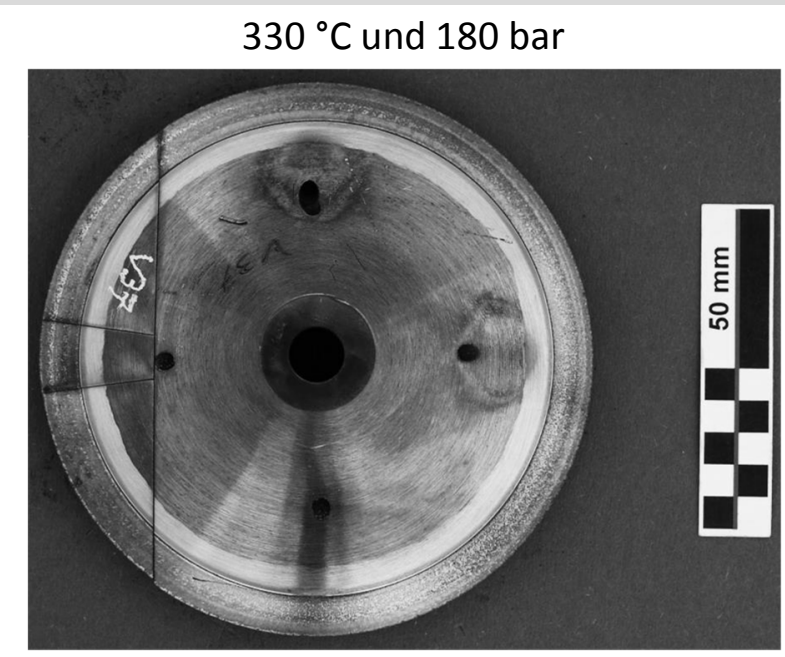

(b)

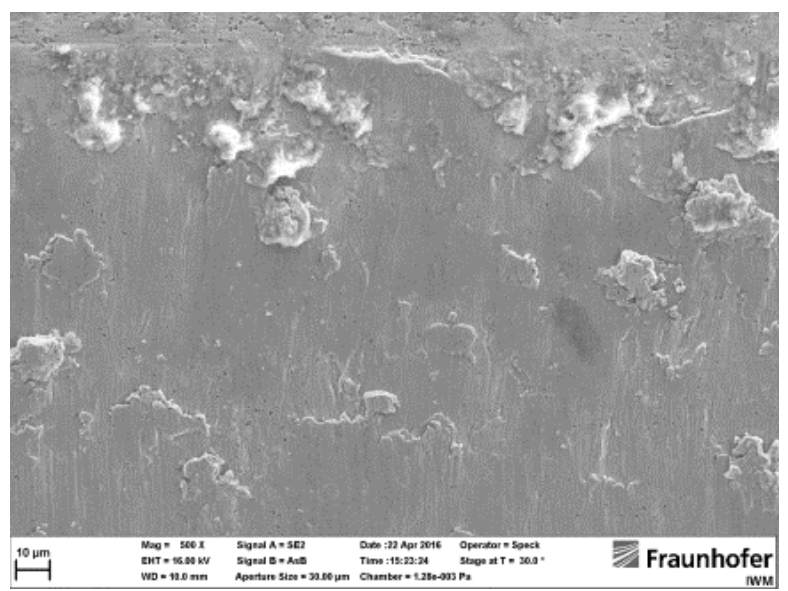

(d)

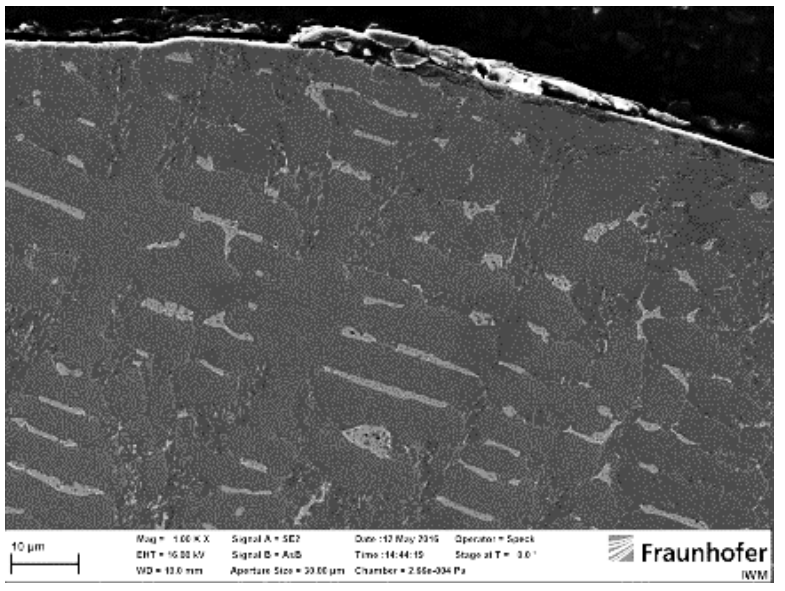

(f)

Abbildung 3.31 Vergleich der Verschleißerscheinungen unter Verbrennungsdruck, Makroaufnahmen von den Ventiltellern (a) und (b), REM-Aufnahmen von den Draufsichten (c) und (d) sowie den zugehörigen Querschliffen (e) und (f) 


\subsubsection{Verschleißrate}

Der Kenngröße Verschleißrate kommt eine entscheidende Bedeutung zur Beurteilung des Verschleißverhaltens $\mathrm{zu}$, da sie ein quantitatives Kriterium zur Vergleichbarkeit von Prüfstandsversuch und befeuertem Motorbetrieb ist. Für die Validierung wurden drei repräsentative Versuche herangezogen, zwei über $100 \mathrm{~h}$ unter $0,6 \mathrm{~m} / \mathrm{s}$ bei RT und $430^{\circ} \mathrm{C}$ sowie ein Langzeitversuch über $447 \mathrm{~h}$ unter $1,2 \mathrm{~m} / \mathrm{s}$ bei $450^{\circ} \mathrm{C}$. Abbildung 3.32 veranschaulicht die Tiefenprofile mit den zugehörigen Verschleißraten. Mit Erhöhung der Temperatur von RT auf $430{ }^{\circ} \mathrm{C}$ steigt die Verschleißrate von 0,06 auf $1,60 \mathrm{~mm}^{3} / \mathrm{h}$ an. Dies entspricht in etwa dem Faktor 27.

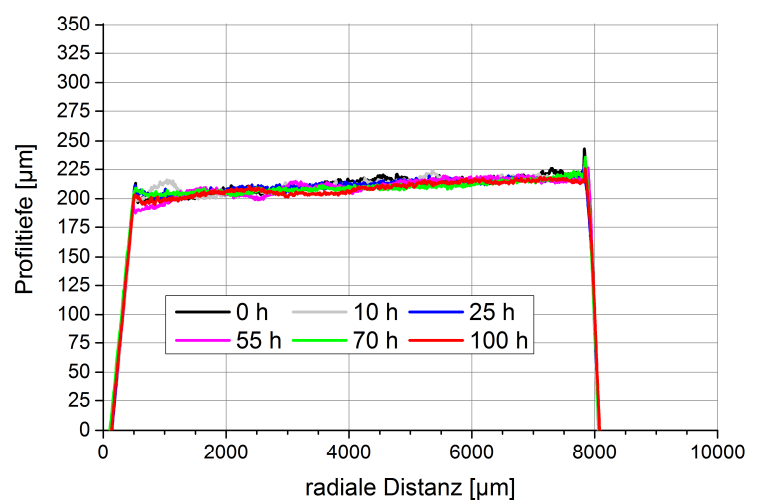

(a), $0,6 \mathrm{~m} / \mathrm{s}$ bei RT

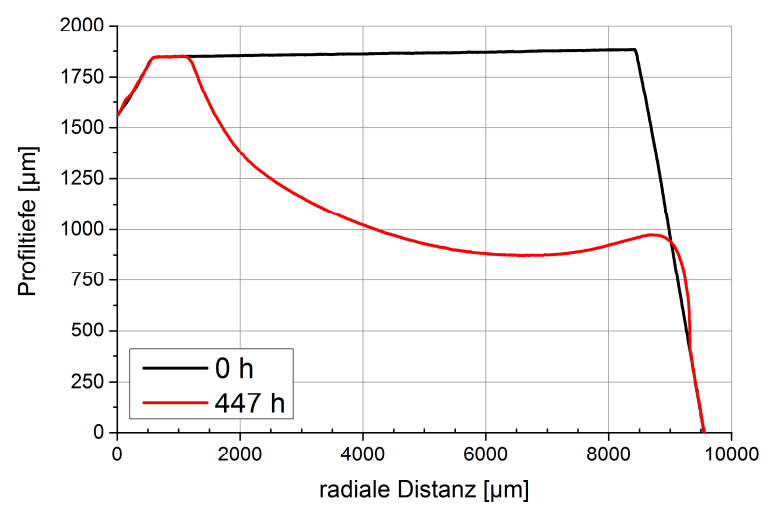

(c), $1,2 \mathrm{~m} / \mathrm{s}$ bei $450^{\circ} \mathrm{C}$

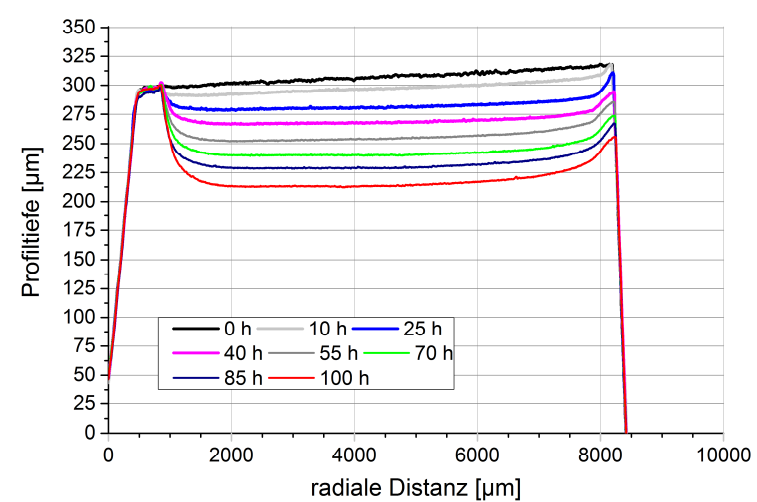

(b), $0,6 \mathrm{~m} / \mathrm{s}$ bei $430{ }^{\circ} \mathrm{C}$

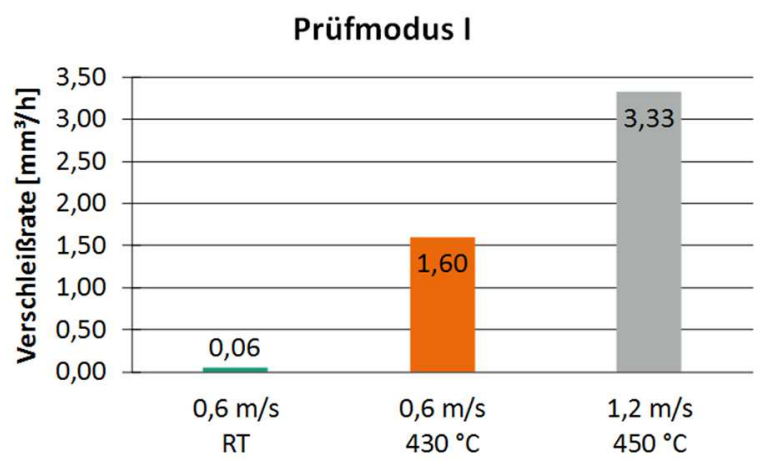

(d)

Abbildung 3.32: Verschleißprofile unter $0,6 \mathrm{~m} / \mathrm{s}$ bei Raumtemperatur (a) und $430^{\circ} \mathrm{C}$ (b) sowie unter $1,2 \mathrm{~m} / \mathrm{s}$ bei $450^{\circ} \mathrm{C}$ (c) und zugehörige Verschleißraten (d)

Betrachtet man die Verschleißprofile unter $0,6 \mathrm{~m} / \mathrm{s}$ bei $430{ }^{\circ} \mathrm{C}$, kann ein lineares Verhalten beobachtet werden. So bewirkt die Verdopplung der Schließgeschwindigkeit von 0,6 auf 1,2 m/s eine Erhöhung der Verschleißrate um den Faktor 2. Im Vergleich mit den Feldteilen liegen die ermittelten Verschleißraten in derselben Größenordnung. Die Verschleißrate unter $1,2 \mathrm{~m} / \mathrm{s}$ mit 3,33 $\mathrm{mm}^{3} / \mathrm{h}$ ist vergleichbar groß zur Probe A3 mit 3,81 mm $3 / \mathrm{h}$. Daher kann der Prüfstand in diesem wichtigen Kriterium als validiert angesehen werden, weil Verschleißrate und Verschleißerscheinungsformen von Prüfstand- und Motorversuch übereinstimmen. 


\section{$4 \quad$ Erste Ergebnisse aus den Prüfstandsversuchen}

Charakteristisches Merkmal des neuentwickelten VVPs ist die getrennte Simulation der Ventilphasen Schließen und Verbrennungsdruck auf Komponentenebene für Großgasmotoren. In diesem Kapitel sollen die ersten Ergebnisse zum Ventilschließen und Verbrennungsdruck vorgestellt werden, wobei die beiden Hauptparameter 3 -stufig bei $380^{\circ} \mathrm{C}$ an Luft variiert wurden. Die vorgestellten Versuche sind typische Versuche, die repräsentativ für die untersuchten tribotechnischen Werkstoffe Stellite ${ }^{\mathrm{TM}} 12$ und Pleuco $12 \mathrm{MV}$ sind. In Tabelle 4.1 ist eine Übersicht der zwei Versuchsreihen wiedergegeben. Für die Prüfungen wurden Serienkomponenten aus mehreren freigegebenen Produktionslosen verwendet. Der Ausgangszustand ist chargenabhängig und streut somit innerhalb der Spezifikationen. Bei der Interpretation der Versuchsergebnisse ist dieser Umstand zu berücksichtigen. Stichproben zur Härteprüfung verdeutlichten, dass der Ausgangszustand der tribotechnischen Werkstoffe variierte. Es wurden Streubreiten für die Stellite ${ }^{\mathrm{TM}}$ 12-Panzerung und dem SR aus Pleuco 12 MV von 3 bzw. 7 HRC im Sitzbereich ermittelt.

Die Mikrostrukturanalyse der Verschleißproben wurde, wie im Kapitel 2.2.1 beschrieben, an drei Positionen durchgeführt. Die ausgewählten Aufnahmen sind repräsentative Aufnahmen für die jeweilige Belastung an Luft nach 100 Versuchsstunden.

Tabelle 4.1: Übersicht der ersten zwei Versuchsreihen zum Ventilschließen und Verbrennungsdruck

\begin{tabular}{|c|c|c|c|c|}
\hline & Nr. & $\begin{array}{l}\text { Schließgeschwindigkeit } \\
{[\mathrm{m} / \mathrm{s}]}\end{array}$ & $\begin{array}{c}\text { Temperatur } \\
{\left[{ }^{\circ} \mathrm{C}\right]}\end{array}$ & $\begin{array}{c}\text { Atmosphäre } \\
{[1]}\end{array}$ \\
\hline \multirow{3}{*}{$\begin{array}{l}\text { Prüfmodus I, } \\
\text { Ventilschließen }\end{array}$} & 1 & 0,2 & \multirow{3}{*}{380} & \multirow{3}{*}{ Luft } \\
\hline & 2 & 0,6 & & \\
\hline & 3 & 1,0 & & \\
\hline & & $\begin{array}{l}\text { Verbrennungsdruck } \\
\text { [bar] }\end{array}$ & $\begin{array}{c}\text { Temperatur } \\
{\left[{ }^{\circ} \mathrm{C}\right]}\end{array}$ & $\begin{array}{c}\text { Atmosphäre } \\
{[1]}\end{array}$ \\
\hline \multirow{3}{*}{$\begin{array}{l}\text { Prüfmodus II, } \\
\text { Verbrennungsdruck }\end{array}$} & 4 & 140 & \multirow{3}{*}{380} & \multirow{3}{*}{ Luft } \\
\hline & 5 & 180 & & \\
\hline & 6 & 220 & & \\
\hline
\end{tabular}




\subsection{Ventilschließen an Luft bei $380^{\circ} \mathrm{C}$}

\subsubsection{Quantitative Verschleißanalyse}

Abbildung 4.1 veranschaulicht vergleichend die Ergebnisse der Profilometrie nach jeweils $100 \mathrm{~h}$. Die Schließgeschwindigkeit wurde in den drei Stufen 0,2, 0,6 und 1,0 m/s variiert.
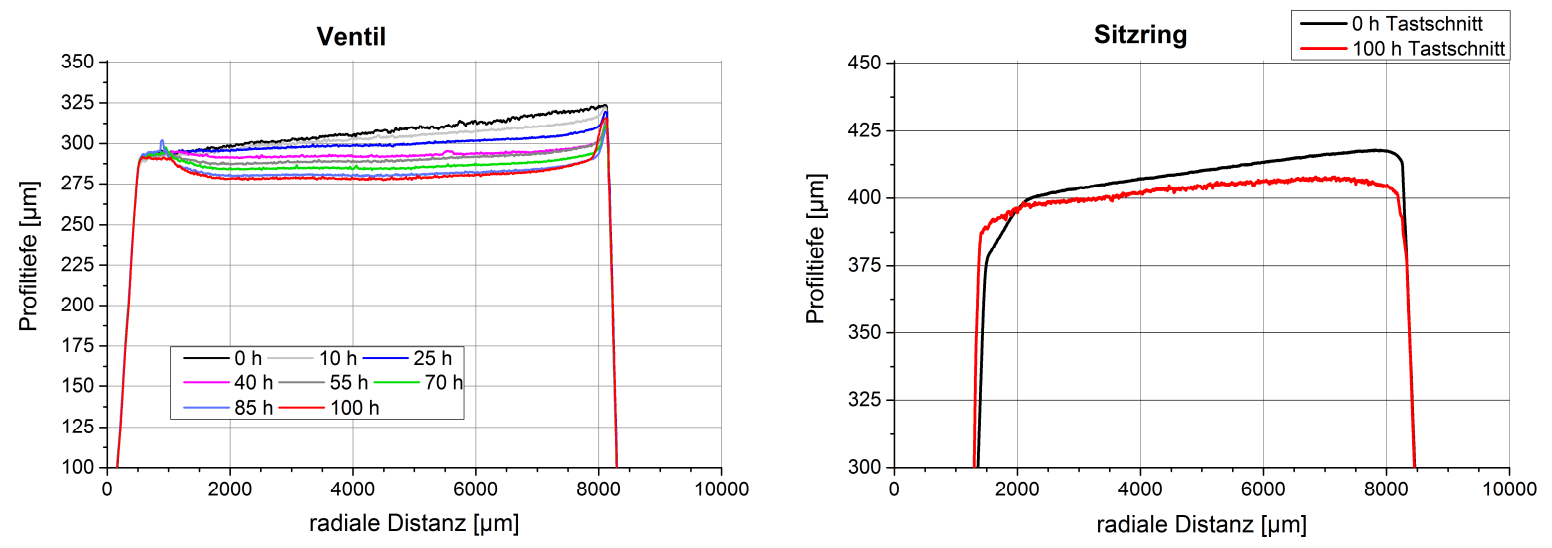

$0,2 \mathrm{~m} / \mathrm{s}$
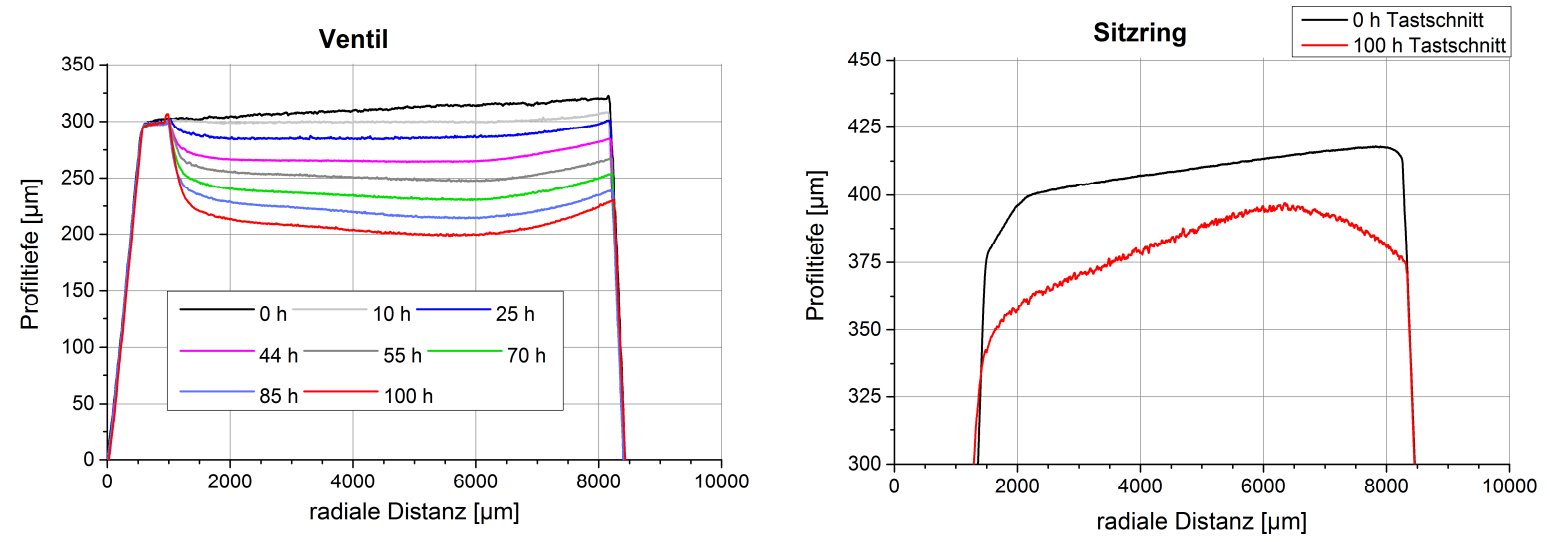

$0,6 \mathrm{~m} / \mathrm{s}$
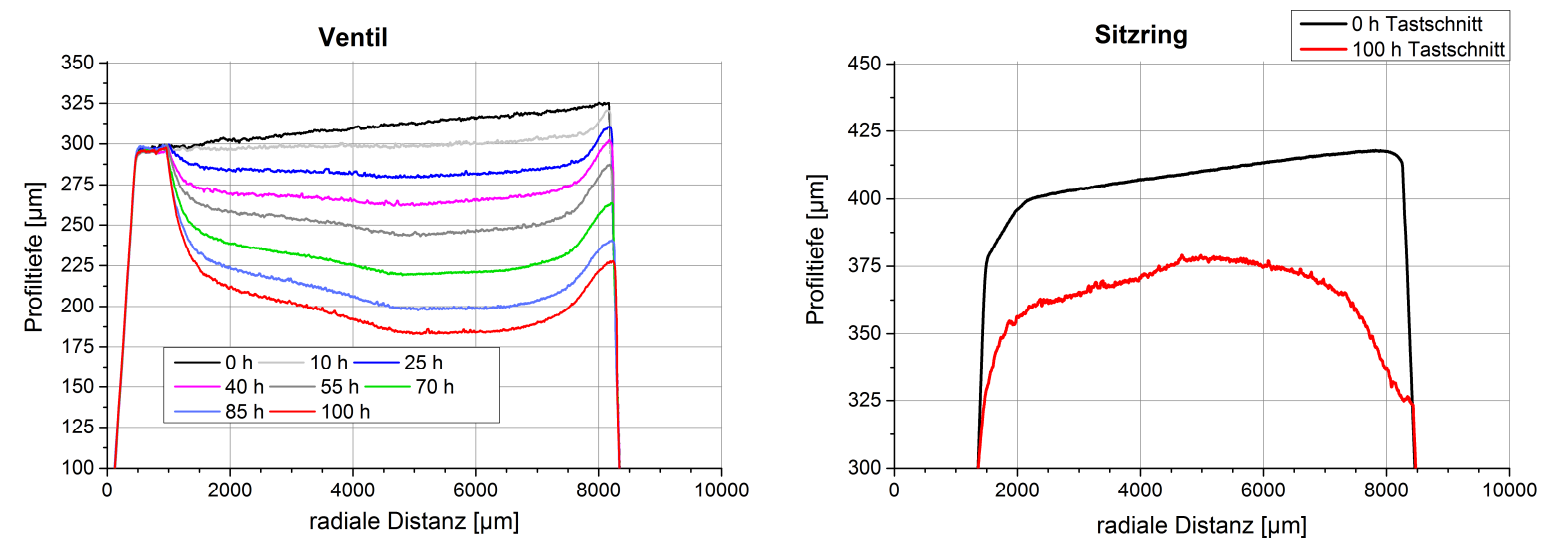

$1,0 \mathrm{~m} / \mathrm{s}$

Abbildung 4.1: Verschleißtiefenprofile von VS (links) und SR (rechts) bei 3-stufiger Variation der Schließgeschwindigkeit unter 0,2, 0,6 und 1,0 m/s bei $380^{\circ} \mathrm{C}$ an Luft 
Zur Orientierung, die SID von VS und SR befinden sich an den Positionen 0 bzw. $1750 \mu \mathrm{m}$. Betrachtet man die Abstände der VS-Profile, so scheinen diese äquidistant zu sein und deuten somit auf ein lineares Verschleißverhalten hin. Unter allen drei Laststufen erreicht die Verschleißmarkenbreite die nominellen Sitzbreiten an VS und SR. In Abhängigkeit von der Belastung scheinen sich die Verschleißprofiltiefen über die Zeitachse zu verändern. Steigt die Schließgeschwindigkeit, vergrößern sich die Profiltiefen und damit der Verschleiß. Ein ausgeprägter Einlaufverschleiß kann bei allen drei Laststufen nicht beobachtet werden. Somit ist ein Einlaufen des Tribosystems VS/SR nicht zu erkennen. Interessant sind die Konturänderungen mit steigender Belastung an VS und SR. Der Werkstoffverlust erfolgt nicht gleichmäßig über die Verschleißmarkenbreite. Vielmehr verändern sich die Tiefenprofile von VS und SR von parallel zu konkav bzw. konvex. Augenscheinlich verhalten sich die Verschleißflächen komplementär zueinander. Mit steigender Schließgeschwindigkeit wird eine ausgeprägte Konkavität im VS-Profil sichtbar. Im Vergleich der VS-Profile unter 0,6 und $1,0 \mathrm{~m} / \mathrm{s}$ ist ein vergleichbar großer Profilrückgang in radialer Distanz von $2000 \mathrm{~mm} \mathrm{zu}$ erkennen, wobei im weiteren Verlauf die Konkavität in Richtung des SADs mit steigender Belastung zunimmt. Vergleicht man die SR-Profile unter 0,6 und 1,0 m/s, scheint sich die Konvexität in Abängigkeit von der Belastung zu verschieben. So befindet sich das Profilmaxima unter $0,6 \mathrm{~m} / \mathrm{s}$ in einer radialen Distanz von ca. $7500 \mu \mathrm{m}$, wohingegen das Maxima unter $1,0 \mathrm{~m} / \mathrm{s}$ in einer Distanz von ca. $5000 \mu \mathrm{m}$ lokalisiert ist. Im Vergleich der SID und SAD von VS und SR ist eine Verrundung für alle SR-Profile festzustellen, wobei mit steigender Schließgeschwindigkeit ein größerer Profilrückgang erkennbar wird. Am SAD der VS weist eine sich ausbildende Profilspitze bei größerem Profilrückgang auf eine beginnende Gratbildung hin.

In Abbildung 4.2 sind die ermittelten Komponentenverschleißraten von VS und SR dargestellt. Für die Versuche unter $0,6 \mathrm{~m} / \mathrm{s}$ ist ein Fehlerindikator aus drei Versuchen bestimmt worden. So betragen Ober- und Untergrenze für die VS 0,72 bzw. 0,63 $\mathrm{mm}^{3} / \mathrm{h}$. Den SR kennzeichnet eine symmetrische Ober-und Untergrenze von $0,24 \mathrm{~mm}^{3} / \mathrm{h}$. VS und SR lassen über alle drei Laststufen keinen gemeinsamen Trend erkennen. Für die VS lässt sich ein steigender Verschleiß mit zunehmender Belastung vermuten. So steigt die Verschleißrate von Laststufe zu Laststufe um den Faktor 2. Hingegen kann für den SR kein Trend im untersuchten Parameterbereich wegen der Verschleißerhöhung von 0,2 auf 0,6 m/s um den Faktor 10 und einer ähnlich großen Verschleißrate unter 0,6 und 1,0 m/s erkannt werden. Aufgrund des großen Fehlerindikators der VS ist eine schärfende Aussage über die Art des Verschleißverhaltens, linear oder exponentiell, nicht ableitbar. Zusammenfassend ist für die VS eine Signifikanz des Einflussfaktors Ventilschließen im betrachteten Parameterbereich festzustellen. Für den SR gilt dies eingeschränkt im Bereich von 0,2 bis $0,6 \mathrm{~m} / \mathrm{s}$. Die Verschleißrate des Versuchs unter $1,0 \mathrm{~m} / \mathrm{s}$ liegt innerhalb des Fehlerindikators vom Versuch unter $0,6 \mathrm{~m} / \mathrm{s}$. 


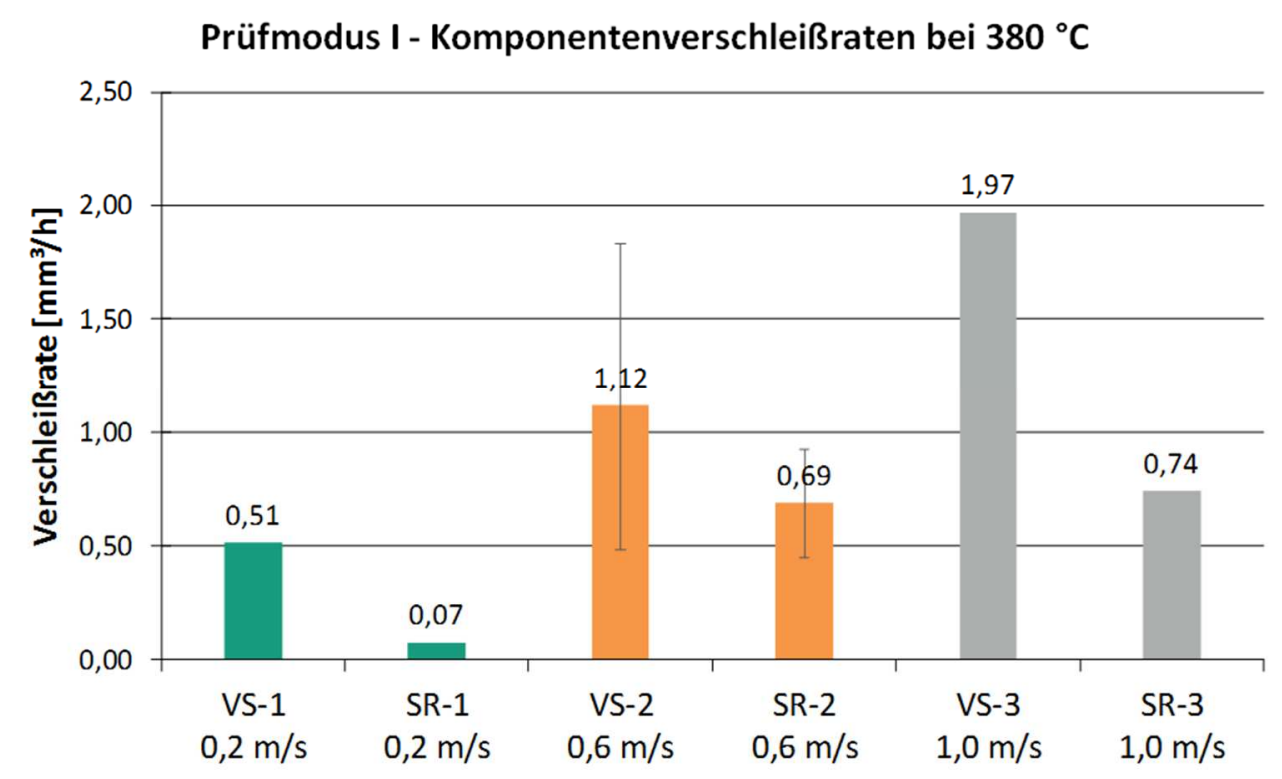

Abbildung 4.2: Komponentenverschleißraten von VS und SR für die Versuchsreihe Ventilschließen unter $0,2,0,6$ und $1,0 \mathrm{~m} / \mathrm{s}$ bei $380^{\circ} \mathrm{C}$ an Luft

Abbildung 4.3 illustriert die Systemverschleißraten zum Ventilschließen. Eine Änderung der anteiligen Komponentenverschleißbeträge von 0,2 auf $0,6 \mathrm{~m} / \mathrm{s}$ ist zu erkennen. So steigt der SR-Verschleiß anteilig von 12 auf $38 \%$. Von 0,6 auf 1,0 m/s ändert sich hingegen das Verhältnis nicht. Diese Beobachtung korreliert mit den ermittelten Verschleißraten vom SR unter 0,6 und $1,0 \mathrm{~m} / \mathrm{s}$. In Bezug auf den Systemverschleiß dominiert der Komponentenverschleiß VS über alle Belastungstufen. Somit kann die VS als bestimmende Größe des Tribosystems VS/SR angesehen werden. Betrachtet man die Systemverschleißbeträge, so scheint sich der Verschleiß des Tribosystems mit steigender Schließgeschwindigkeit zu vergrößern.

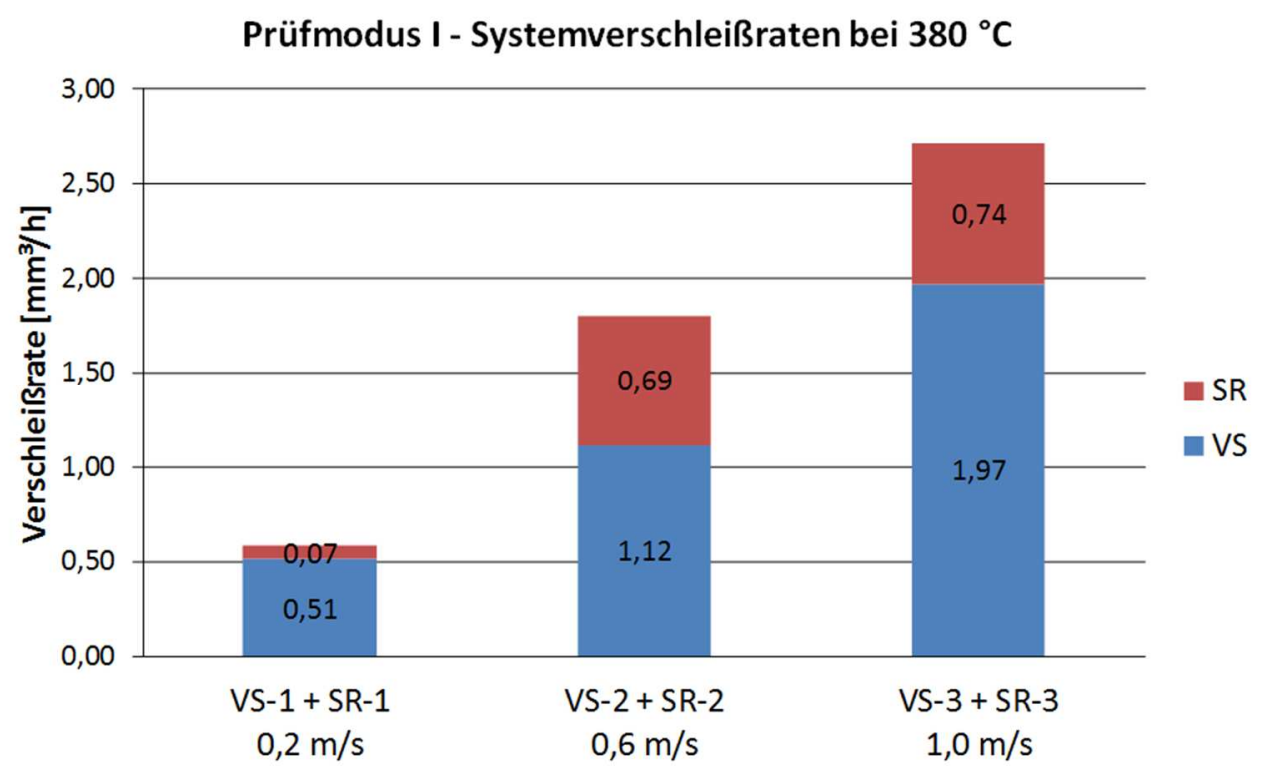

Abbildung 4.3: Systemverschleißraten zur Versuchsreihe Ventilschließen unter 0,2, 0,6 und $1,0 \mathrm{~m} / \mathrm{s}$ bei $380^{\circ} \mathrm{C}$ an Luft 


\subsubsection{Mikrostrukturanalyse}

Abbildung 4.4 veranschaulicht die REM-Aufnahmen von VS und SR in Draufsicht und Querschliff unter 0,2 m/s. Die VS-Oberfläche präsentiert sich unterschiedlich geglättet.

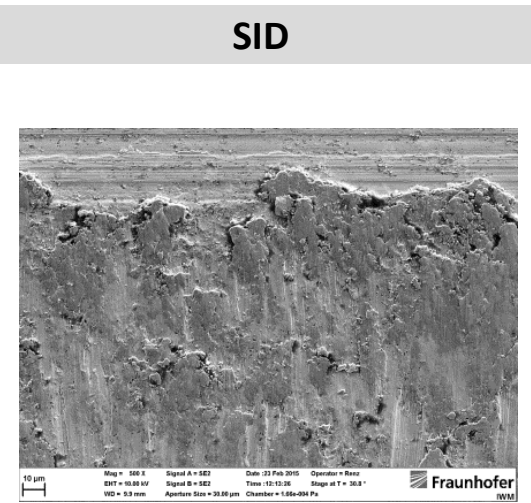

(a)

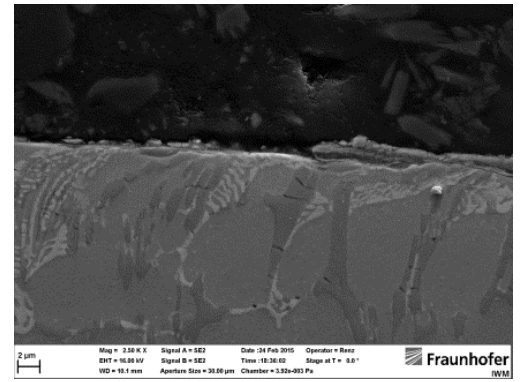

(d)

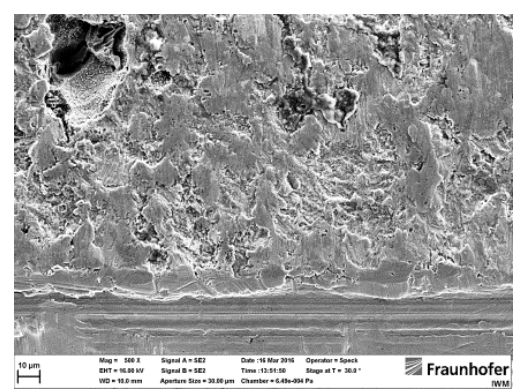

(g)

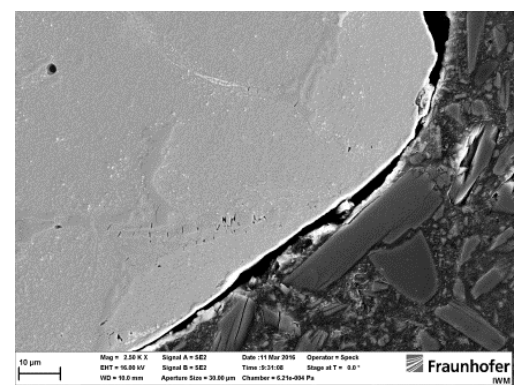

(j)

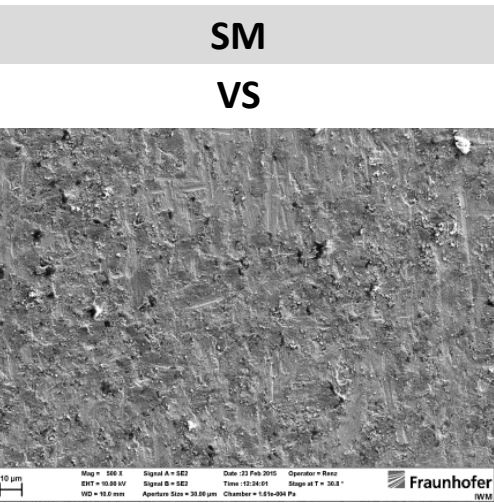

(b)

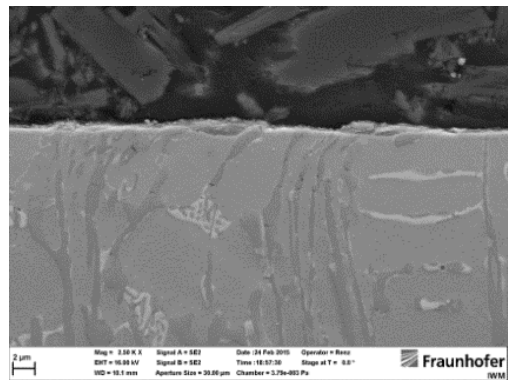

(e)

SR

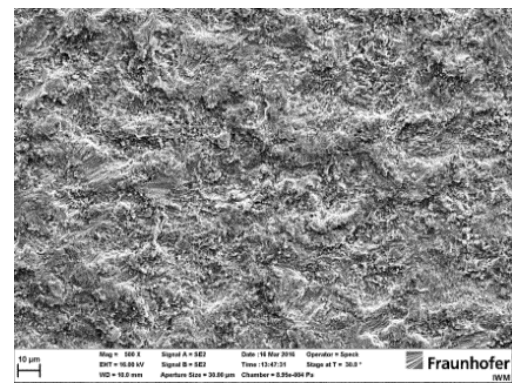

(h)

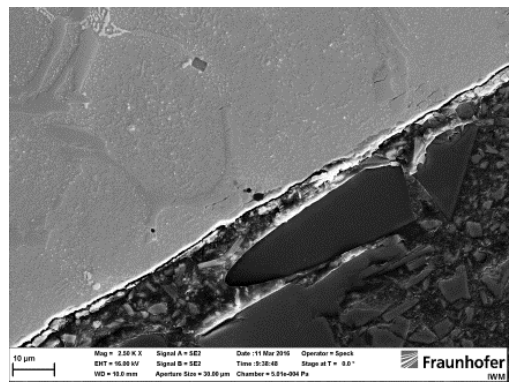

(k)
SAD

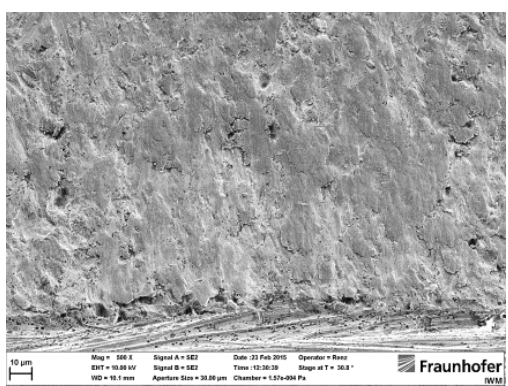

(c)

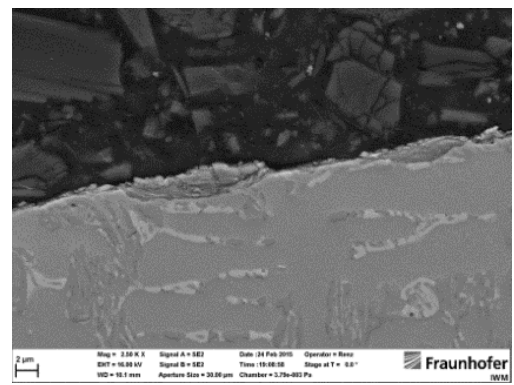

(f)

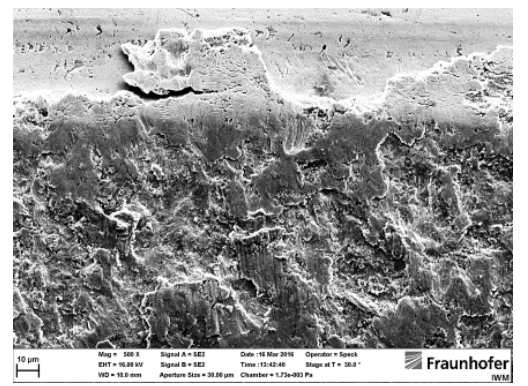

(i)

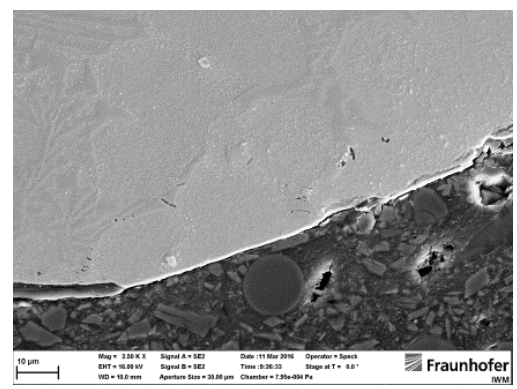

(l)

Abbildung 4.4: REM-Aufnahmen von VS und SR in Draufsicht und Querschliff unter $0,2 \mathrm{~m} / \mathrm{s}$ bei $380^{\circ} \mathrm{C}$ an Luft; 500x (a) - (c) und (g) - (i), 1500x (d) - (f) und (j) - (I) 
Die Verschleißerscheinungsformen der VS stellt sich mit Schuppen, Kuppen und Löchern dar, wobei Schuppen an SID und SM sowie Mulden mit Kuppen am SAD dominieren. Am SID erscheinen die Schuppen wie aufgeschoben. Die Schuppigkeit im Bereich der SM ist erkennbar kleiner, weshalb die Draufsicht aufgerauter erscheint. In den Querschliffen ist in allen Bereichen ein Dehnungsgradient in Verbindung mit Verschleißpartikeln zu sehen, wobei die dendritischen Karbidstrukturen in der tribologisch beanspruchten Randschicht zerbrochen wurden. Auf der Kontaktfläche der VS ist lokal eine Triboschicht zu finden. Im Vergleich dazu weist der Gegenkörper SR in allen drei Bereichen eine rauer erscheinende Kontaktfläche auf. Schuppen und Kuppen sind an SID und SAD zu erkennen. Vielmehr dominieren Mulden in der SM und Ausbrüche an SID und SAD die Verschleißspur. Scherzonen oder Hinweise auf eine Triboschicht sind in den Querschliffen nicht zu beobachten.

In Abbildung 4.5 sind die REM-Aufnahmen von VS und SR unter 0,6 m/s dargestellt. Die Verschleißerscheinungen präsentieren sich im Vergleich zu $0,2 \mathrm{~m} / \mathrm{s}$ in Draufsicht und Querschliff mit Gemeinsamkeiten und Unterschieden. Bei der VS finden sich Schuppen und Kuppen an SID und SM. Als Gemeinsamkeit können aufgeschobene Schuppen am SID beobachtet werden, wobei die Schuppenausprägung unter $0,6 \mathrm{~m} / \mathrm{s}$ geglätteter erscheint. Am SAD dominiert im Gegensatz dazu augenscheinlich eine feinmuldige Oberfläche die Verschleißspur. In den Querschliffen kann in allen Bereichen der Probe ein Dehnungsgradient beobachtet werden. Gleichfalls sind wie unter $0,2 \mathrm{~m} / \mathrm{s}$ zerbrochene Karbide in der tribologisch beanspruchten Randschicht zu sehen. Der Gegenkörper SR präsentiert sich mit ähnlichen Verschleißerscheinungen wie unter $0,2 \mathrm{~m} / \mathrm{s}$ jedoch in unterschiedlichen Bereichen. Im Vergleich zu 0,2 m/s finden sich in den Bereichen SID und SM Schuppen und Kuppen. Insbesondere in der SM bestimmen Schuppen statt Mulden wie unter $0,2 \mathrm{~m} / \mathrm{s}$ die Verschleißspur. Ferner dominieren am SAD unter $0,6 \mathrm{~m} / \mathrm{s}$ Mulden und Ausbrüche das Erscheinungsbild. Eine Triboschicht konnte weder am Grund- noch am Gegenkörper beobachtet werden.

Abbildung 4.6 veranschaulicht die REM-Aufnahmen von VS und SR unter 1,0 m/s. Die Werkstoffantwort der VS verdeutlicht eine unterscheidbare Schädigung der Mikrostruktur im Vergleich zu den Versuchen unter 0,2 und 0,6 m/s. Schuppen und Kuppen bestimmen das oberflächliche Erscheinungsbild der Verschleißspur. Als Gemeinsamkeit können am SID aufgeschobene Schuppen beobachtet werden. Daneben sind am SAD Schuppen und Kuppen zu sehen, wobei Schuppen die Verschleißspur in diesem Bereich augenscheinlich dominieren. Am SAD kann erstmalig ein Riss erkannt werden. Der Bereich der SM wird vergleichbar zu $0,6 \mathrm{~m} / \mathrm{s}$ von Kuppen und Schuppen bestimmt, wobei Kuppen das Bild sichtbar dominieren. Ein Dehnungsgradient kann in allen Querschliffen beobachtet werden. Gleichfalls wurden die dendritischen Karbidstrukturen in der tribologisch beanspruchten Randschicht im Vergleich zu den Versuchen unter 0,2 und 0,6 m/s zerbrochen. Darüber hinaus kann am SAD eine mechanisch gemischte Randschicht bestehend aus feinzerbrochenen Karbiden und metallischer Matrix beobachtet werden. Auf der Kontaktfläche der VS sind eine Vielzahl von Verschleißpartikeln zu sehen. Der Gegenkörper SR präsentiert sich in den Draufsichten deutlich unterschiedlich im Vergleich zu den Versuchen unter 0,2 und $0,6 \mathrm{~m} / \mathrm{s}$. Das Erscheinungsbild der beiden Bereiche SM und SAD wird von einer deutlich eingeglätteten und 
feinmuldigen Oberfläche bestimmt. Vereinzelte Löcher und Ausbrüche sind an der Oberfläche zu sehen. Hingegen sind im Bereich SID aufgeschobene Schuppen zu erkennen. Im Querschliff bestätigt sich diese plastische Erscheinung. So wurde Werkstoff plastisch über den SID hinaus verschoben. Verschleißpartikel und Risse in den Karbiden sowie der Matrix bestimmen die Erscheinung in diesem Bereich erkennbar.

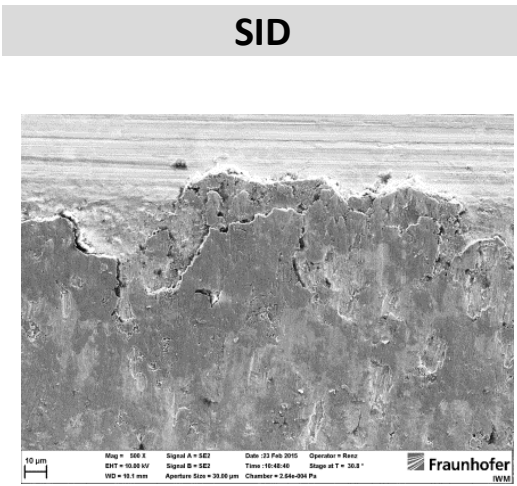

(a)

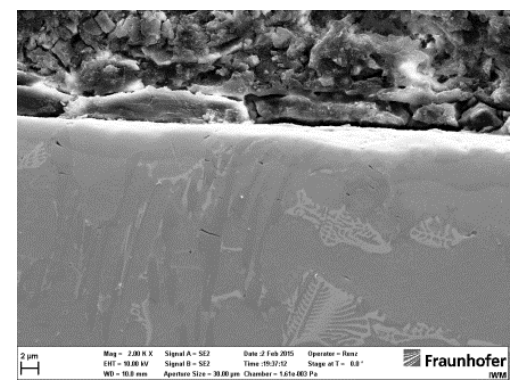

(d)

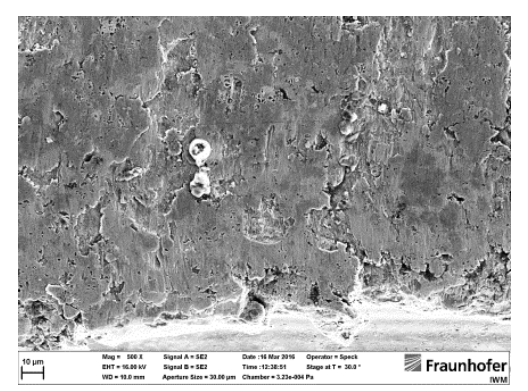

(g)

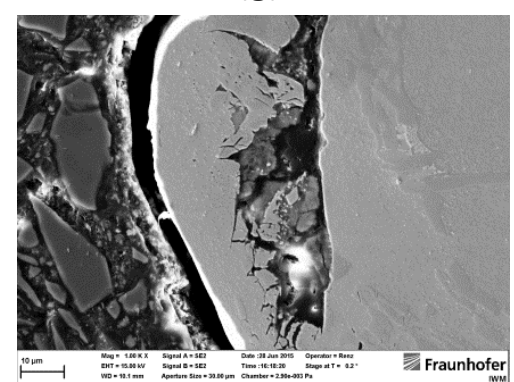

(j)

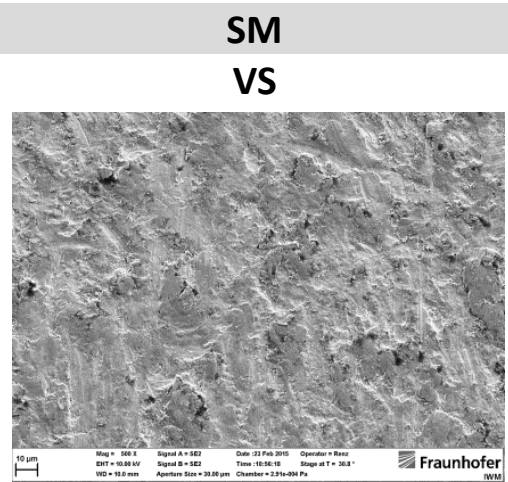

(b)

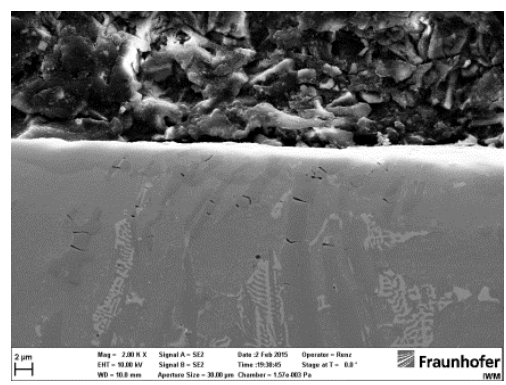

(e)

SR

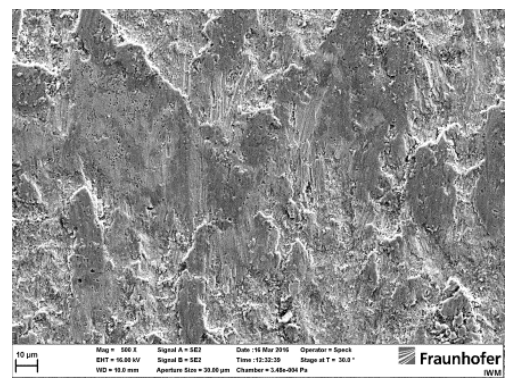

(h)

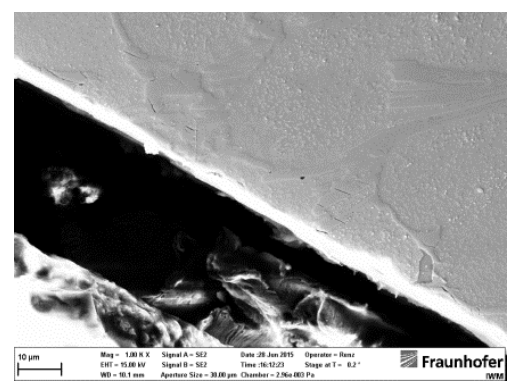

(k)
SAD

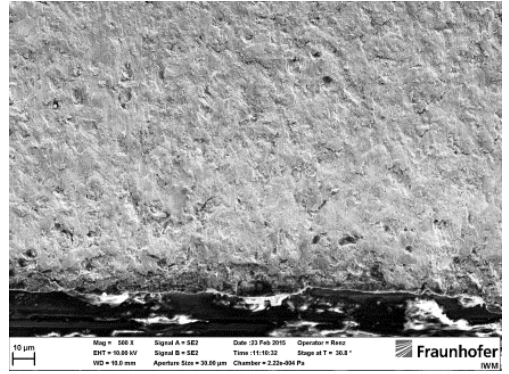

(c)

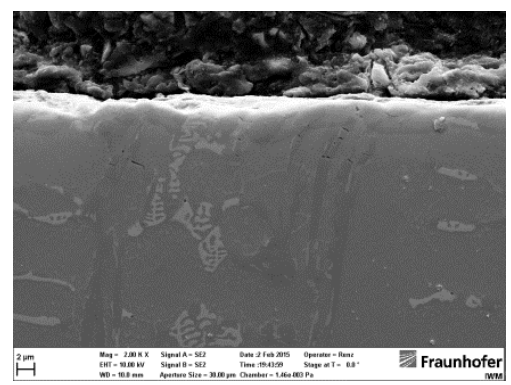

(f)

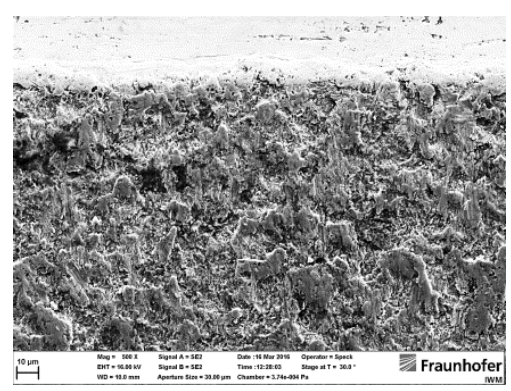

(i)

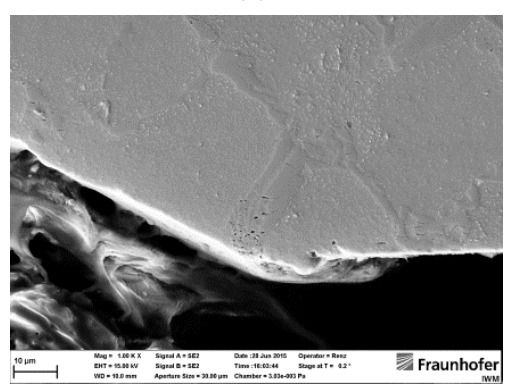

(I)

Abbildung 4.5: REM-Aufnahmen von VS und SR in Draufsicht und Querschliff unter $0,6 \mathrm{~m} / \mathrm{s}$ bei $380^{\circ} \mathrm{C}$ an Luft; 500x (a) - (c) und (g) - (i), 1500x (d) - (f) und (j) - (I) 


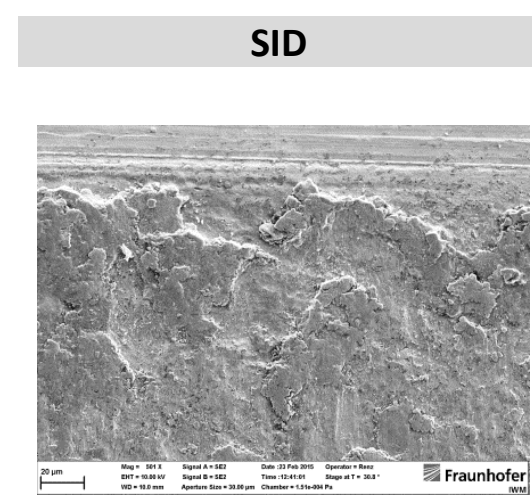

(a)

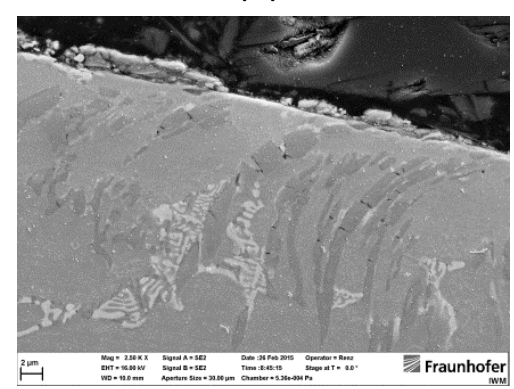

(d)

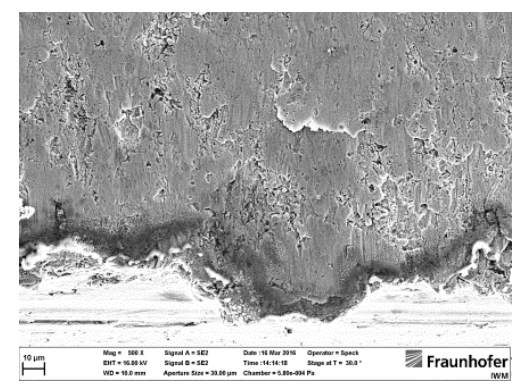

(g)

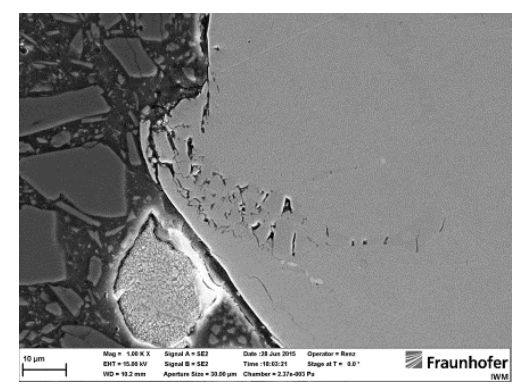

(j)

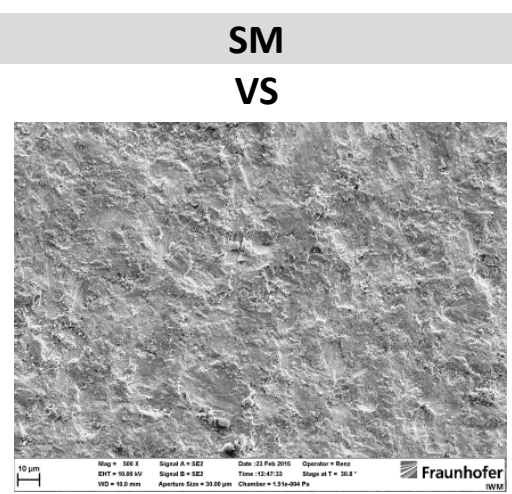

(b)

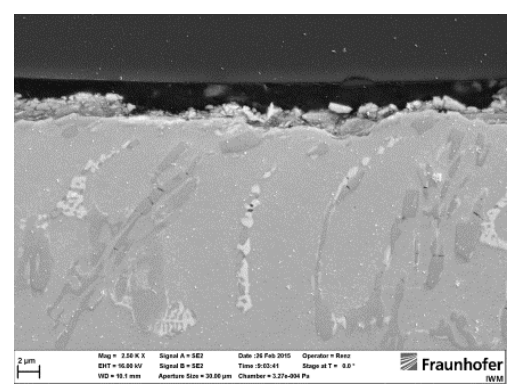

(e)

SR

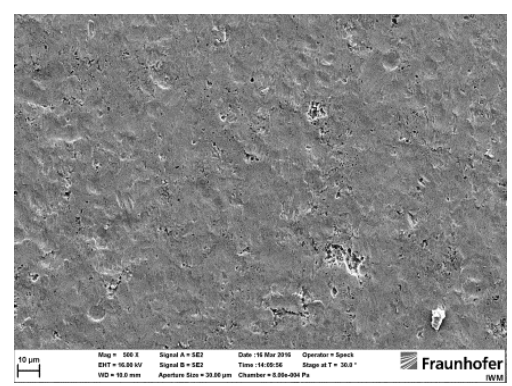

(h)

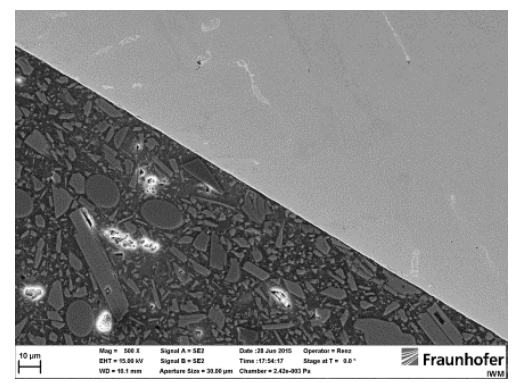

(k)
SAD

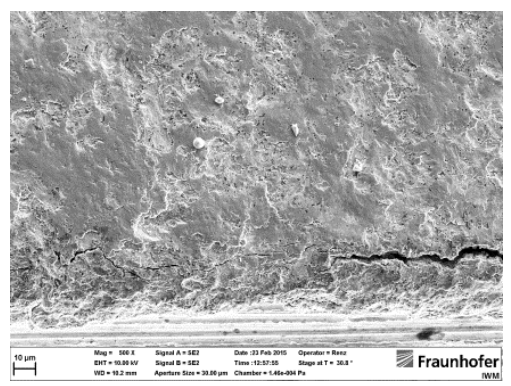

(c)

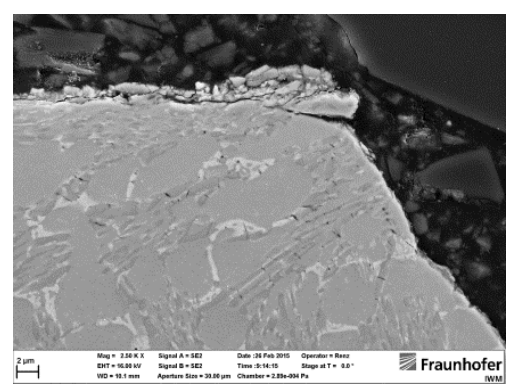

(f)

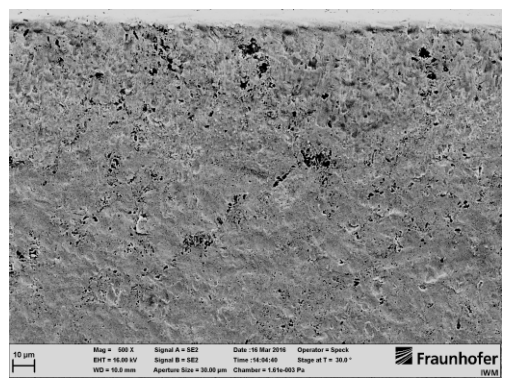

(i)

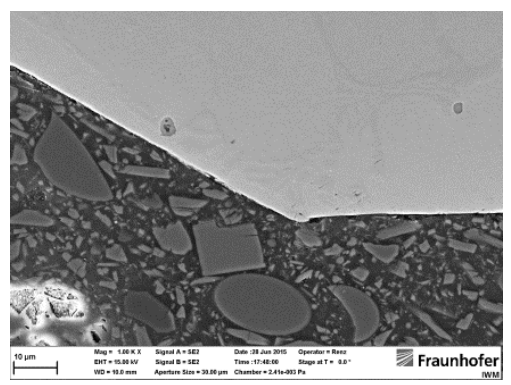

(I)

Abbildung 4.6: REM-Aufnahmen von VS und SR in Draufsicht und Querschliff unter $1,0 \mathrm{~m} / \mathrm{s}$ bei $380^{\circ} \mathrm{C}$ an Luft; 500x (a) - (c) und (g) - (i), 1500x (d) - (f) und (j) - (I) 


\subsection{Verbrennungsdruck an Luft bei $380{ }^{\circ} \mathrm{C}$}

\subsubsection{Quantitative Verschleißanalyse}

Abbildung 4.7 veranschaulicht vergleichend die Ergebnisse der Profilometrie nach jeweils 100 h. Der Verbrennungsdruck wurde in den drei Stufen 140, 180 und 220 bar variiert. Dies war druckäquivalent mit den Stempelkräften 80, 105 und 130 kN.
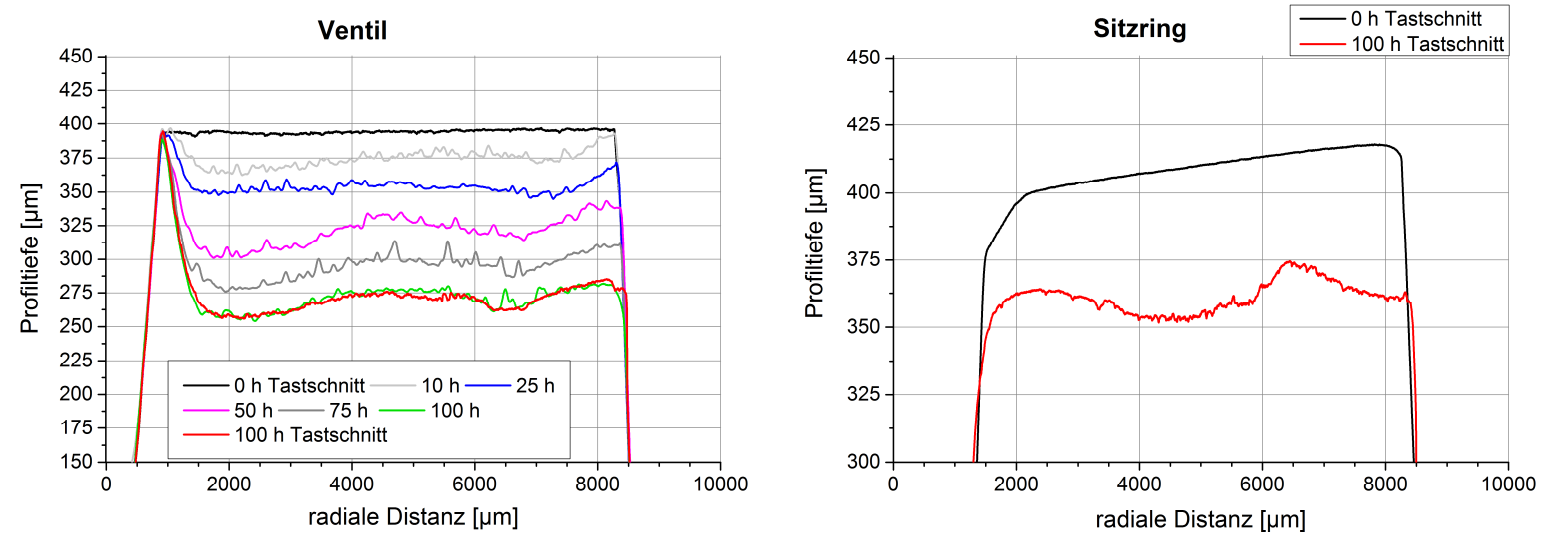

$140 \operatorname{bar}(\equiv 80 \mathrm{kN})$
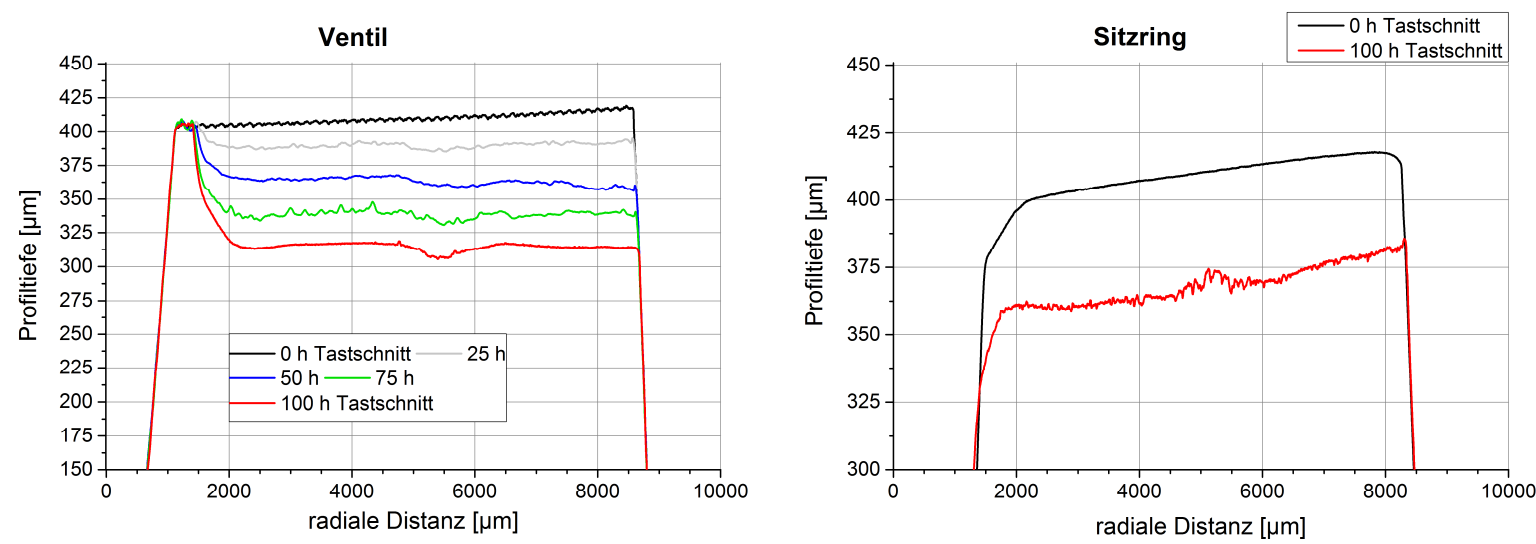

$180 \operatorname{bar}(\equiv 105 \mathrm{kN})$
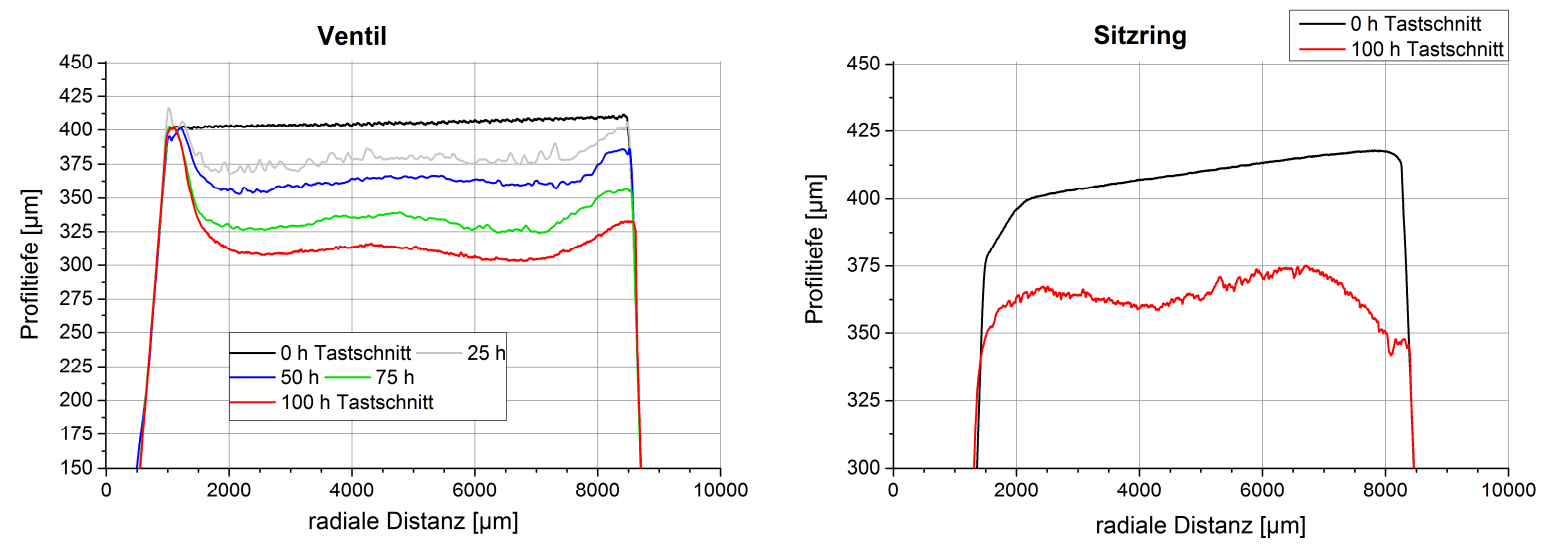

$220 \operatorname{bar}(\equiv 130 \mathrm{kN})$

Abbildung 4.7: Verschleißprofile von VS (links) und SR (rechts) bei 3-stufiger Variation des Verbrennungsdrucks unter 140, 180 und 220 bar bei $380^{\circ} \mathrm{C}$ an Luft 
Die ungeglättet erscheinenden Profilverläufe des Versuchs unter 140 bar erklären sich aus dem Wechsel der angewendeten Methode zur Verschleißprofilmessung im Verlauf der Versuchsreihen. Zur Reduktion des Messaufwands wurde vom Tastschnittverfahren auf die 3D-Laserscan-Farbmikroskopie mittels Abdruckmasse gewechselt. Zur Orientierung, an den Positionen 500 bzw. $1750 \mu \mathrm{m}$ befinden sich die SID von VS und SR. Vergleicht man die Abstände der VS-Profile, so scheinen diese analog zum Ventilschließen äquidistant zu sein und weisen ebenso auf ein lineares Verschleißverhalten hin. Ähnlich wie beim Ventilschließen nehmen die Profiltiefen über die Zeitachse zu. Absolut betrachtet, kann hingegen ein anderes Verschleißverhalten beobachtet werden. So nehmen im Kontrast zum Ventilschließen die Profiltiefen mit steigender Belastung ab. Über alle Laststufen konnte für die betrachtete Versuchsdauer kein erhöhter Anfangsverschleiß gemessen werden. Somit kann in Analogie zum Ventilschließen kein Einlaufen des Tribosystems VS/SR festgestellt werden. Bemerkenswert sind die Konturänderungen in den Profilen von VS und SR mit steigender Belastung. Betrachtet man die SR-Profile, so ist allen Profilen nach $100 \mathrm{~h}$ eine Verrundung im Bereich des SIDs gemein. In Bezug auf die VS scheint als globaler Trend ein eher gleichmäßiger Werkstoffverlust über die ganze Verschleißmarkenbreite typisch zu sein. Die ausgeprägte Konkavität des Profils der VS mit entsprechender Konvexität des Profils vom SR wie unter Ventilschließen kann nicht beobachtet werden. Für die Versuche der VS unter 140 und 220 bar kann bereits nach 10 bzw. $25 \mathrm{~h}$ mit Profilspitzen am SAD ein kleinerer Verschleiß am SAD im Vergleich zur SM festgestellt werden. Dieses Phänomen vergrößert sich mit zunehmender Versuchsdauer. Unter 140 und 220 bar wird im Bereich der SM ein konkaves Profil erkennbar, wohingegen unter 180 bar ein nahezu paralleles Verschleißprofil gemessen wurde. Vergleicht man die Profile von VS und SR über alle drei Belastungsstufen, darf als Gemeinsamkeit eine Komplementarität beobachtet werden. In den konkaven Profilbereichen der VS finden sich konvexe Bereiche des SRs wieder.

Abbildung 4.8 veranschaulicht die ermittelten Komponentenverschleißraten von VS und SR aller drei Laststufen. Analog zum Ventilschließen wurde für die mittlere Laststufe unter 180 bar ein Fehlerindikator aus drei Versuchen ermittelt. Die Obergrenzen für VS und SR betragen 0,16 bzw. $0,87 \mathrm{~mm}^{3} / \mathrm{h}$, die Untergrenzen wurden für VS und SR mit 0,28 bzw. $0,45 \mathrm{~mm}^{3} / \mathrm{h}$ bestimmt. Anzumerken ist, dass sich das relativ große Delta für den SR aus den Verschleißraten von Versuch 1 mit 1,96 $\mathrm{mm}^{3} / \mathrm{h}$ sowie der Versuche 2 und 3 mit 0,66 bzw. $0,64 \mathrm{~mm}^{3} / \mathrm{h}$ ergab. Die Verschleißverhalten der Komponenten VS und SR sind unterschiedlich. Ein globaler Trend ist über die drei Laststufen nicht eindeutig zu erkennen. So kann für die VS von 140 auf 180 bar ein Rückgang um 33 \% festgestellt werden. Hingegen kann von 180 auf 220 bar kein eindeutiger Trend erkannt werden, da die Verschleißrate vom Versuch unter 220 bar mit 1,64 mm³ $/ \mathrm{h}$ im Bereich des Fehlerindikators vom Versuch unter 180 bar liegt. Für den SR kann kein Trend aufgrund des betragsmäßig großen Fehlerindikators abgeleitet werden. Die Frage, inwieweit sich das Verschleißverhalten belastungsabhängig entwickelt, kann aus den Versuchen im betrachteten Parameterbereich nicht beantwortet werden. 


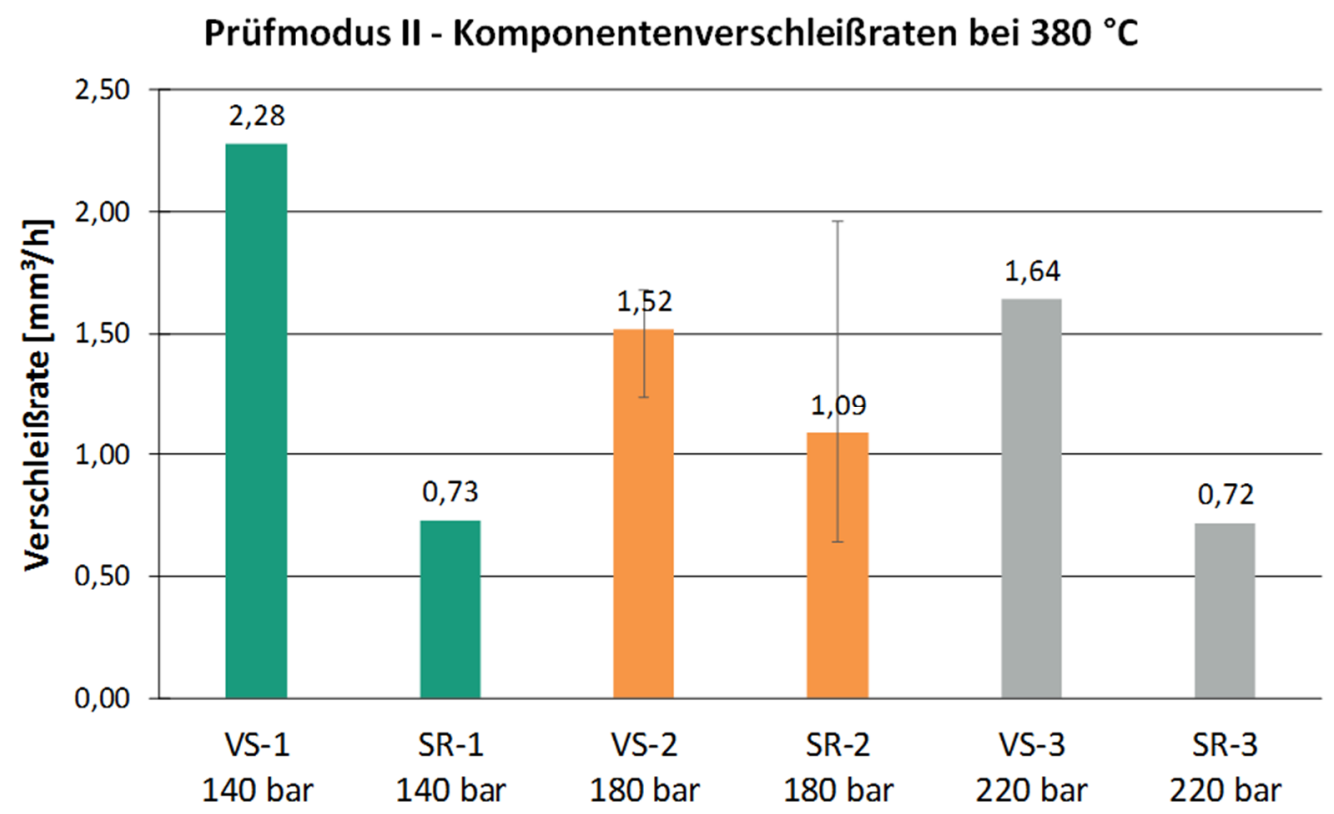

Abbildung 4.8: Komponentenverschleißraten von VS und SR der Versuchsreihe Verbrennungsdruck unter 140,180 und 220 bar bei $380^{\circ} \mathrm{C}$ an Luft

In Abbildung 4.9 sind die Systemverschleißraten zur Versuchsreihe Verbrennungsdruck dargestellt. Man beobachtet von 140 auf 180 bar eine Abnahme des Systemverschleißes, wobei der Komponentenverschleiß SR anteilig von 24 auf $42 \%$ zunimmt. Dieser Trend setzt sich von 180 auf 220 bar nicht fort, vielmehr sinkt der SR-Anteil auf $31 \%$. In Analogie zum Ventilschließen dominiert der Komponentenverschleiß VS den Systemverschleiß. Die VS ist somit die bestimmende Größe unter Verbrennungsdruck. Betrachtet man den Systemverschleiß, so scheint dieser mit steigendem Verbrennungsdruck abzunehmen.

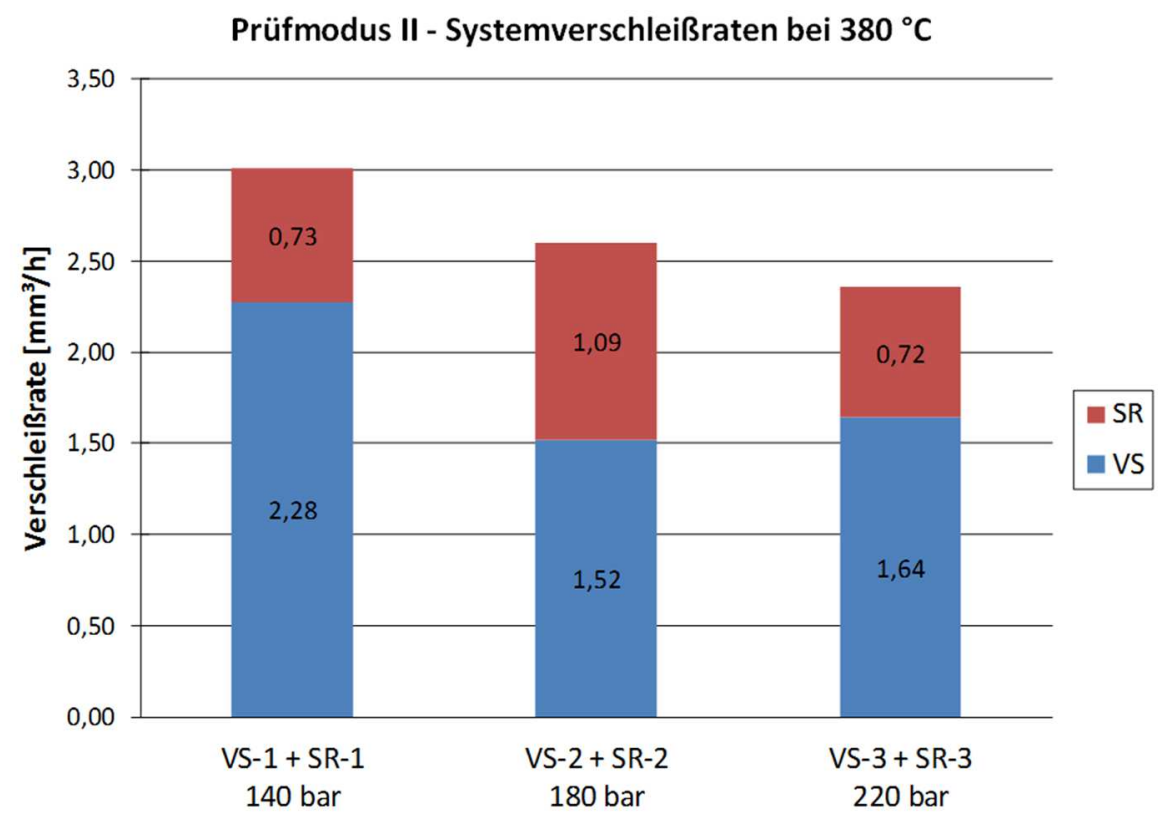

Abbildung 4.9: Systemverschleißraten zur Versuchsreihe Verbrennungsdruck unter 140,180 und 220 bar bei $380^{\circ} \mathrm{C}$ an Luft 
In Abbildung 4.10 ist ein Vergleich der Systemverschleißraten von VS und SR für beide Versuchsreihen Ventilschließen und Verbrennungsdruck dargestellt. In den untersuchten Lastbereichen fällt die gegenläufige Entwicklung der Systemverschleißraten mit steigender Belastung auf. Qualitativ kann man sagen, mit Zunahme der Schließgeschwindigkeit steigt die Systemverschleißrate, hingegen ist eine Abnahme der Systemverschleißrate mit steigendem Verbrennungsdruck festzustellen. Quantitativ erhöht sich der Systemverschleiß um ca. $450 \%$ von 0,2 auf $1,0 \mathrm{~m} / \mathrm{s}$, wohingegen sich der Systemverschleiß von 140 auf 220 bar um ca. $21 \%$ reduziert. Würde ein Motor mit den tribotechnischen Werkstoffen Stellite ${ }^{\mathrm{TM}} 12$ für VS und Pleuco $12 \mathrm{MV}$ für SR unter dem Lastkollektiv $0,2 \mathrm{~m} / \mathrm{s}$ und 140 bar bei $380{ }^{\circ} \mathrm{C}$ an Luft betrieben werden, so würde voraussichtlich der Verschleiß infolge Verbrennungsdruck dominieren. Ändert man das Belastungskollektiv auf 1,0 m/s und 220 bar, hat die im Satz vorher gemachte Aussage keinen Bestand mehr. Vielmehr bestimmen in diesem Fall beide Ventilphasen zu gleichen Teilen den Systemverschleiß. Daher kann für die untersuchten Belastungsbereiche, die typisch für Großmotoren sind, nicht verallgemeinert werden, dass ein Anstieg der Belastungen zu steigendem Verschleiß führt.

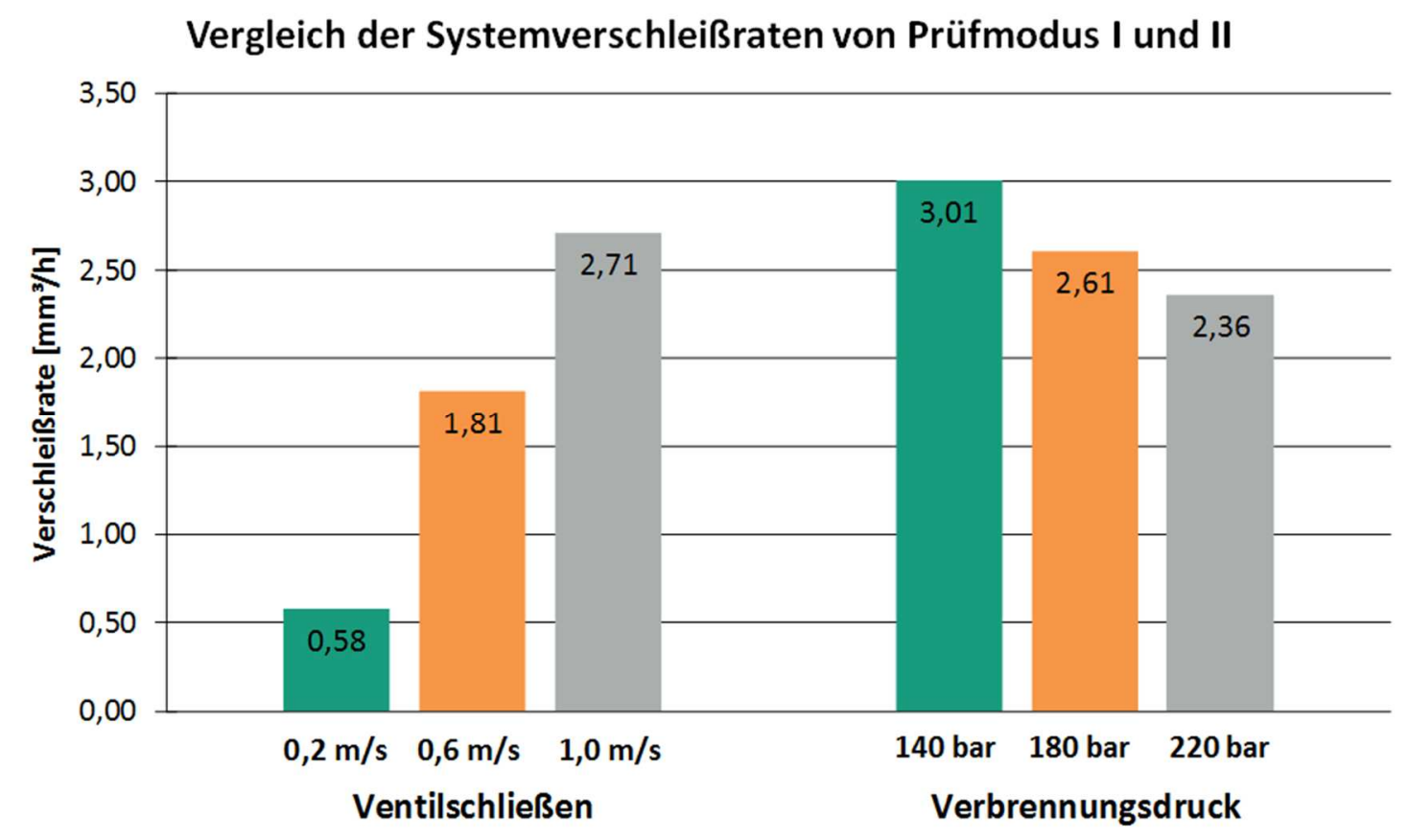

Abbildung 4.10: Vergleich der Systemverschleißraten beider Ventilphasen Ventilschließen und Verbrennungsdruck 


\subsubsection{Mikrostrukturanalyse}

Abbildung 4.11 stellt die REM-Aufnahmen von VS und SR in Draufsicht und Querschliff unter 140 bar dar. Die Verschleißspur der VS präsentiert sich in allen Bereichen der Probe geglättet.

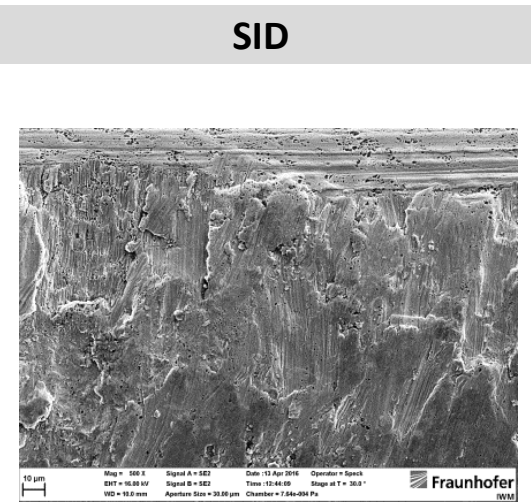

(a)

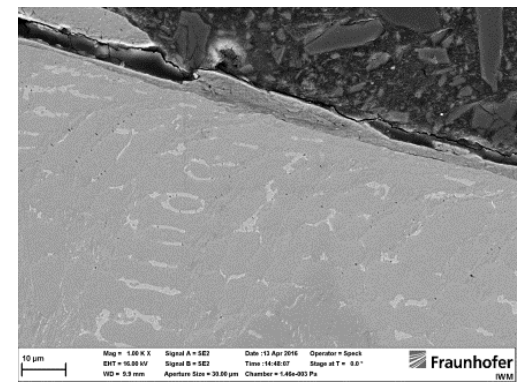

(d)

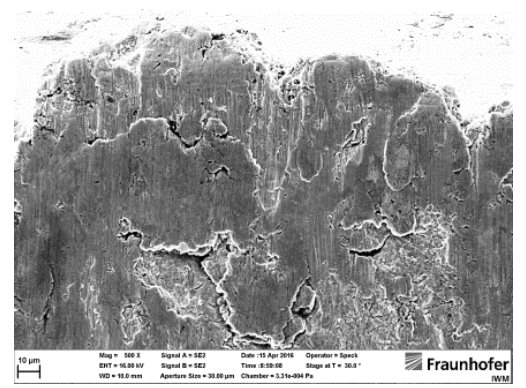

(g)

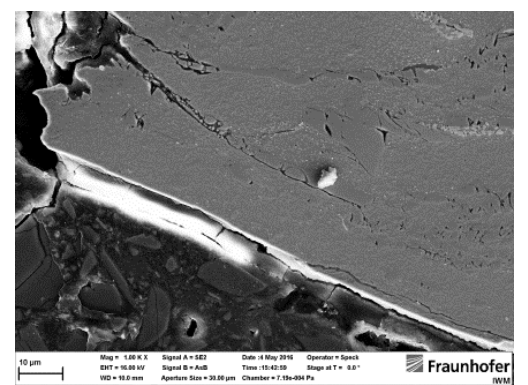

(j)

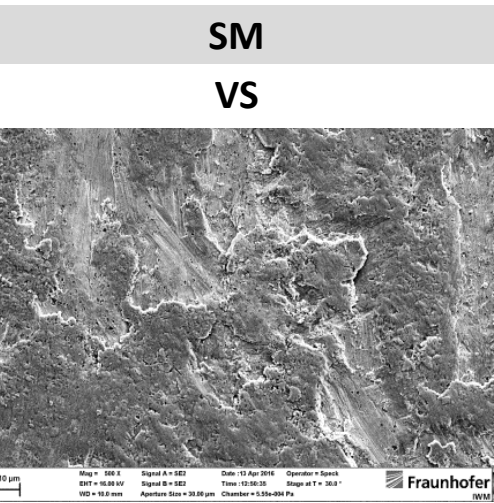

(b)

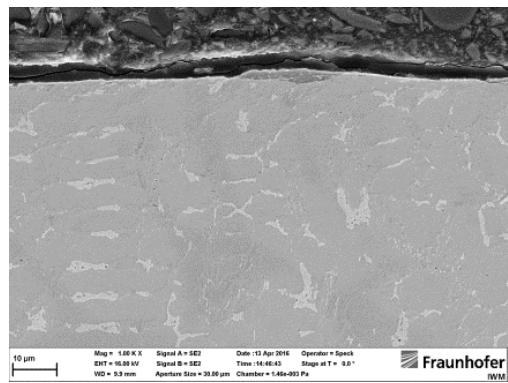

(e)

SR

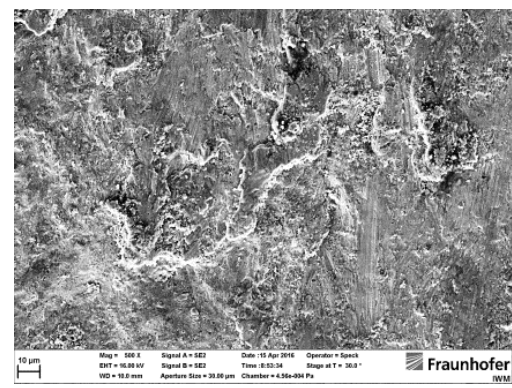

(h)

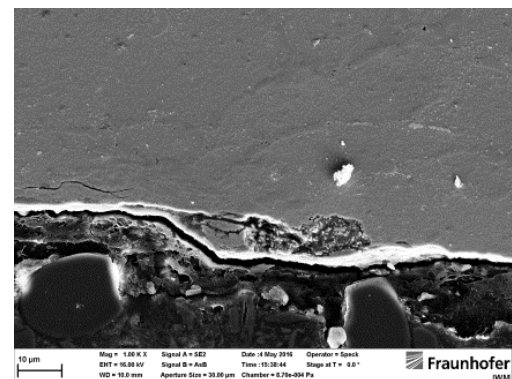

(k)
SAD

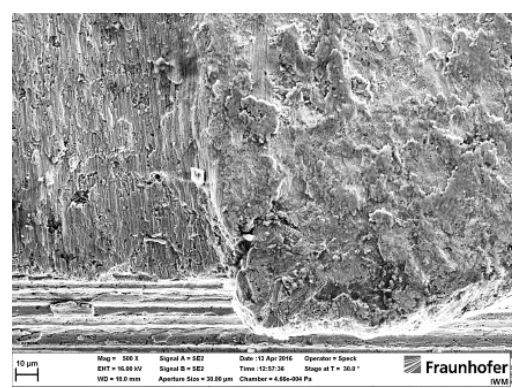

(c)

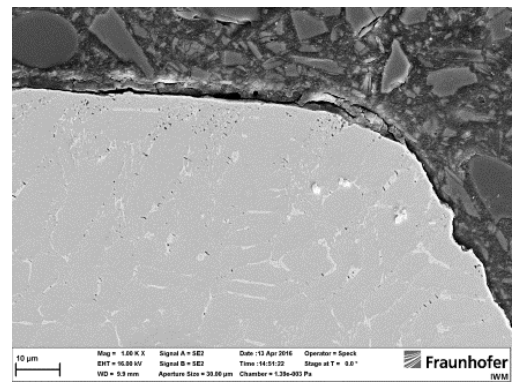

(f)

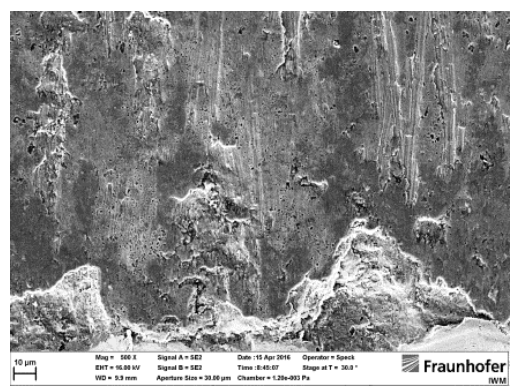

(i)

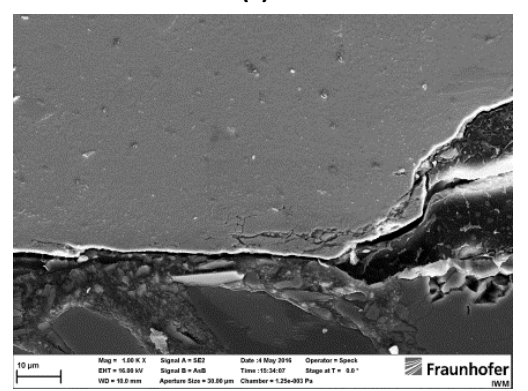

(I)

Abbildung 4.11: REM-Aufnahmen von VS und SR in Draufsicht und Querschliff unter 140 bar bei $380^{\circ} \mathrm{C}$ an Luft; $500 x(\mathrm{a})-(\mathrm{c})$ und (g) - (i), 1000x (d) - (f) und (j) - (I) 
Für den Versuch unter 140 bar stellt sich die Werkstoffantwort der VS mit Schuppen, Löchern und Ausbrüchen an der Kontaktfläche dar, wobei Schuppen im Bereich des SIDs und Schuppen mit Ausbrüchen die Bereiche SM und SAD dominieren. Analog zum Ventilschließen wirken die Schuppen am SID wie aufgeschoben, wobei die Schuppigkeit sichtbar intensiver erscheint. In den Querschliffen der VS kann in allen Bereichen ein Dehnungsgradient beobachtet werden, wobei am SID sowie SM eine lokale Triboschicht zu erkennen ist. Im Bereich des SIDs reicht die plastische Deformation bis in eine Tiefe von ca. $20 \mu \mathrm{m}$. Abbildung 4.12 verdeutlicht zerbrochene Karbide im Dehnungsgradienten. Daneben weist eine EDS-Analyse in der Triboschicht mit einem Fe-Anteil von 26 at.-\% auf eine Werkstoffübertragung vom SR aus Eisenbasis hin. Ferner wurden Co, Cr und C detekiert. Der Nachweis von Co deutet auf eine Kompaktierung von Verschleißpartikeln aus der VS hin. Die Verschleißspur des Gegenkörpers SR ist an SID und SAD von Schuppen sowie in der SM von Ausbrüchen und Kuppen gekennzeichnet. In den Querschliffen sind größere und kleinere parallele Risse zur Oberfläche in allen drei Bereichen zu sehen.
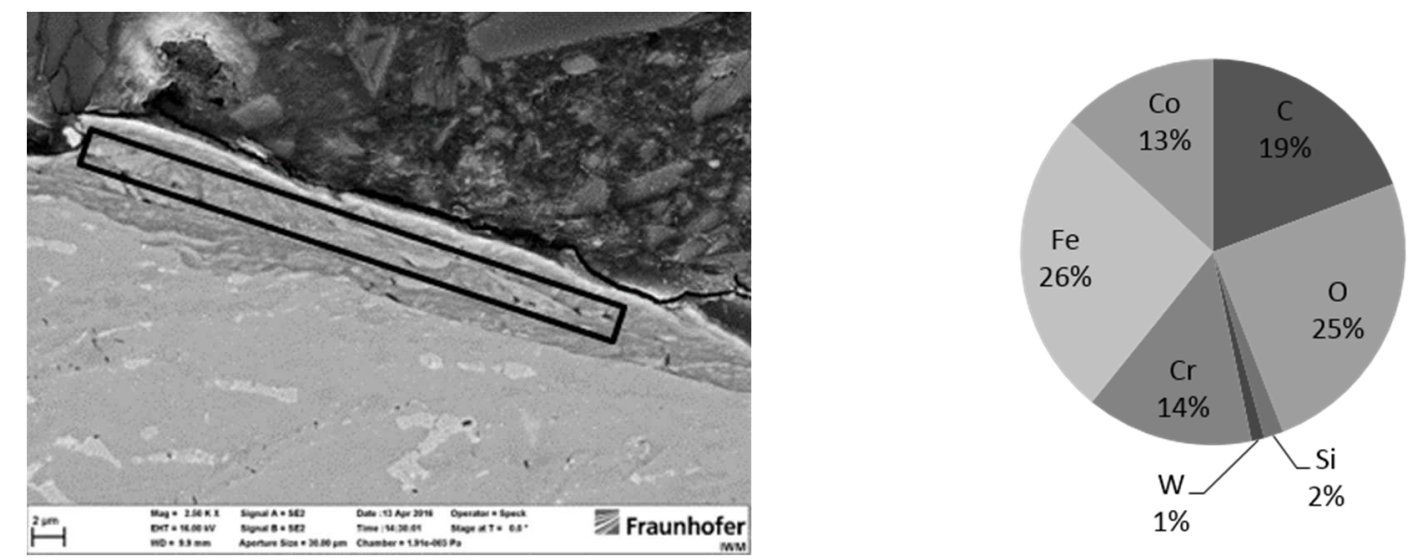

Abbildung 4.12: Detailaufnahme aus Abbildung 4.11 (d) der Triboschicht (links), zugehörige EDS-Analyse in at.-\% des schwarz markierten Rechtecks links (rechts), Fe stammt vermutlich vom Gegenkörper SR aus Fe-Basis

Abbildung 4.13 veranschaulicht die REM-Aufnahmen von VS und SR vom Versuch unter 180 bar. Die Verschleißerscheinungen präsentieren sich vergleichbar zum Versuch unter 140 bar, wobei die Intensität sichtbar kleiner erscheint. Neben Schuppen, Löchern und Mulden bestimmen geglättete Oberflächen die Draufsichten. Im Detail können am SID eine Glättung, im Bereich der SM Schuppen und Löcher sowie am SAD Mulden in Verbindung mit geglätteten Flächen beobachtet werden. Als Gemeinsamkeit mit dem 140 bar Versuch finden sich aufgeschobene Schuppen am SID wieder. Im Querschliff kann ein Dehnungsgradient in allen Bereichen der Probe erkannt werden. Dieser wirkt augenscheinlich weniger ausgeprägt im Vergleich zum Versuch unter 140 bar. Daneben finden sich an der Oberfläche lokal dunkle Bereiche, die auf eine Triboschicht hindeuten. Hier sei angemerkt, dass eine weitere hochauflösende Analytik dieser Bereiche nicht durchgeführt wurde. Der SR lässt ähnliche Verschleißerscheinungen wie unter dem 140 bar Versuch erkennen, wobei die Intensität sichtbar kleiner, ähnlich wie bei der VS unter 140 bar, erscheint. Neben Schuppen und Löchern finden sich Ausbrüche in der Draufsicht. So sind die Bereiche SAD und SID von Schuppen sowie 
Löchern und die SM von Ausbrüchen bestimmt. Die Beobachtung von Ausbrüchen in der Draufsicht wird im Querschliff im Bereich der SM durch lokal begrenzte Vertiefungen gestützt. In allen Querschliffen vom SR sind zerbrochene Karbide zu sehen.

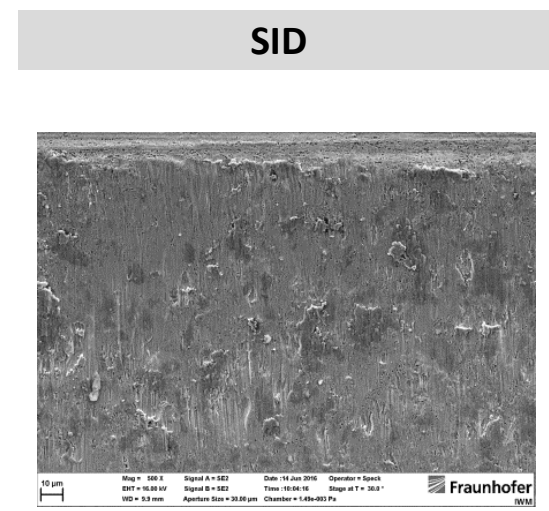

(a)

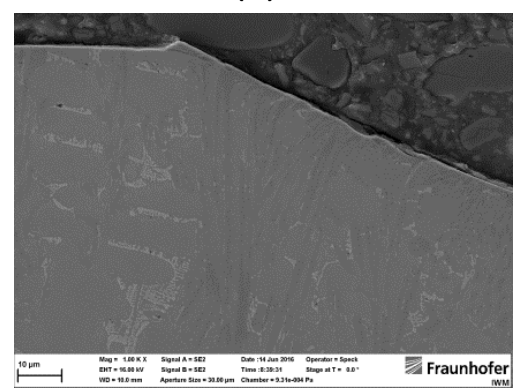

(d)

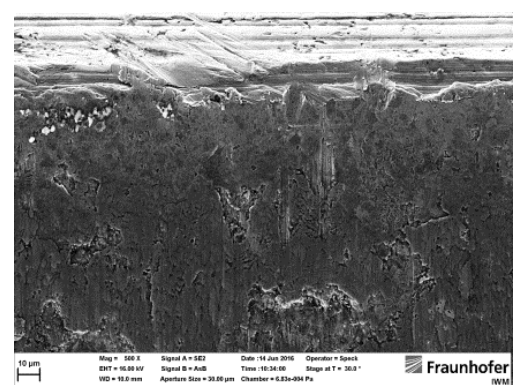

(g)

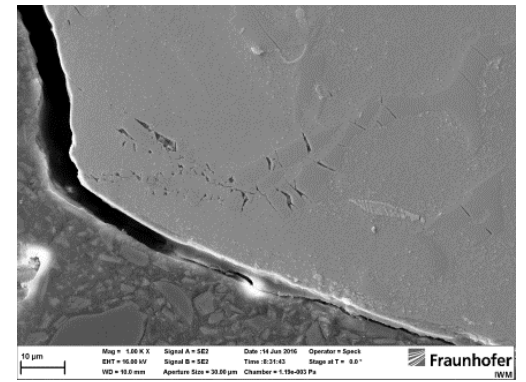

(j)
SM

VS

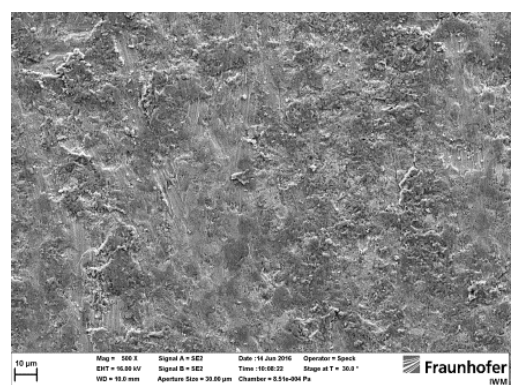

(b)

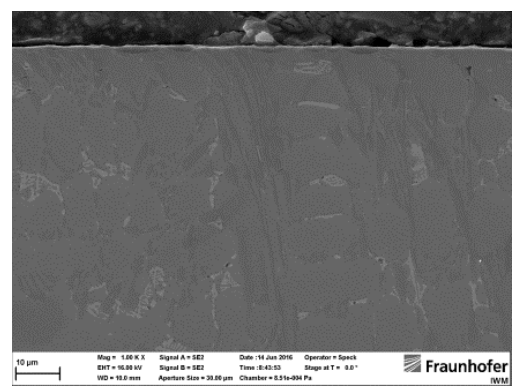

(e)

SR

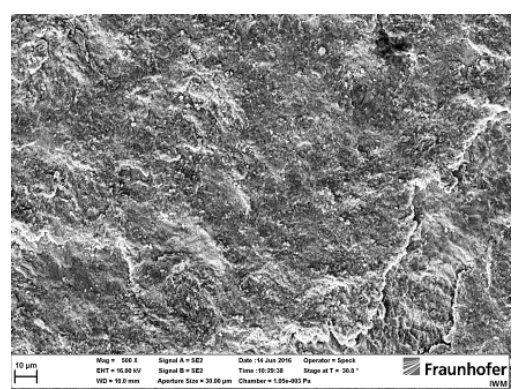

(h)

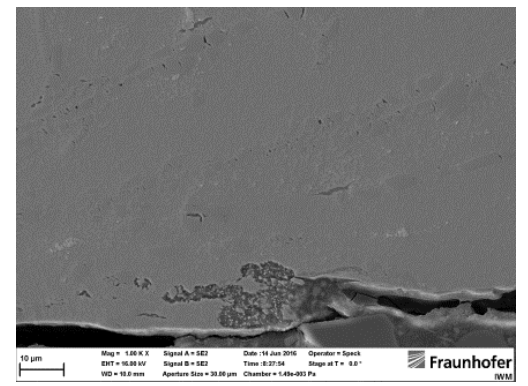

(k)
SAD

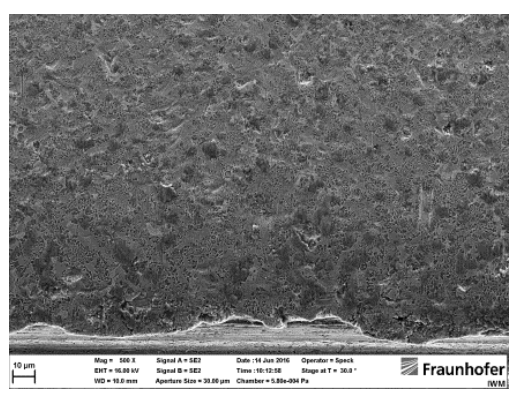

(c)

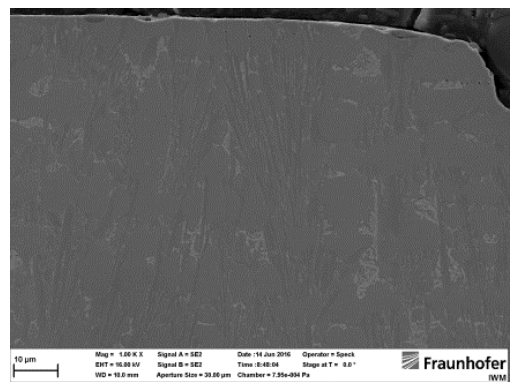

(f)

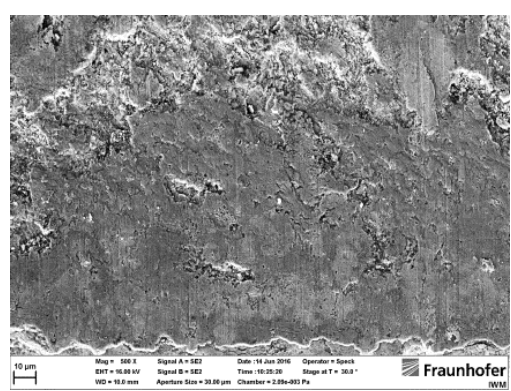

(i)

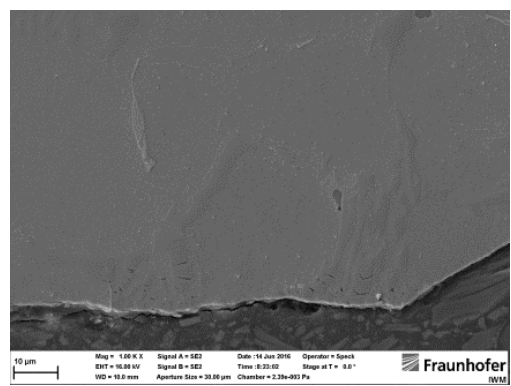

(I)

Abbildung 4.13: REM-Aufnahmen von VS und SR in Draufsicht und im Querschliff unter 180 bar bei $380^{\circ} \mathrm{C}$ an Luft; 500x (a) - (c) und (g) - (i), 1000x (d) - (f) und (j) - (I)

Abbildung 4.14 veranschaulicht die REM-Aufnahmen von VS und SR unter 220 bar. Bei erster Betrachtung fällt an der VS ein ca. $70 \mu \mathrm{m}$ langer Verschleißpartikel am SAD auf (Abbildung 
4.14 (f)). Die Mikrostruktur im und um das Partikel ist plastisch verformt. Ein Dehnungsgradient mit zerbrochenen metallkeramischen Hartphasen bestimmt die tribologische Randschicht in allen Querschliffen. Schuppen und Löcher dominieren allein die Verschleißspur unter dieser höchsten Belastungsstufe von 220 bar, wobei sich die Morphologie in den Bereichen unterscheidet. So beobachtet man kompaktierte Schuppen mit Löchern in der SM und aufgeschobene Schuppen in den Bereichen SID und SAD.

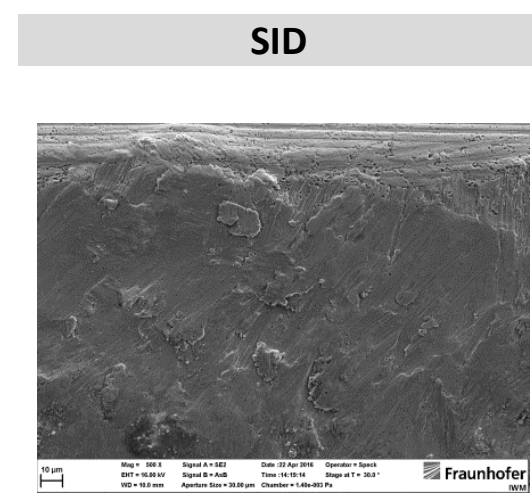

(a)

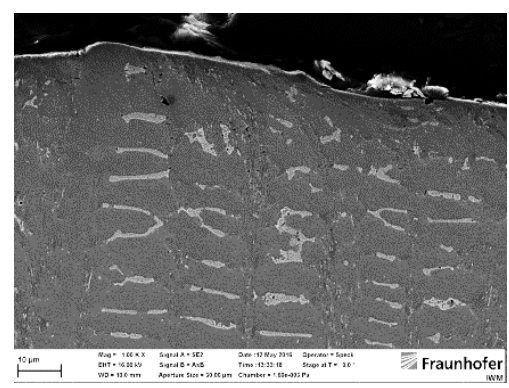

(d)

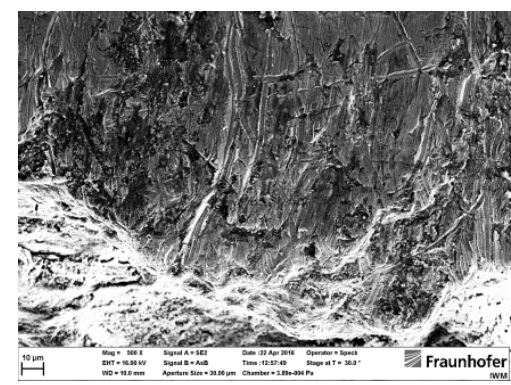

(g)

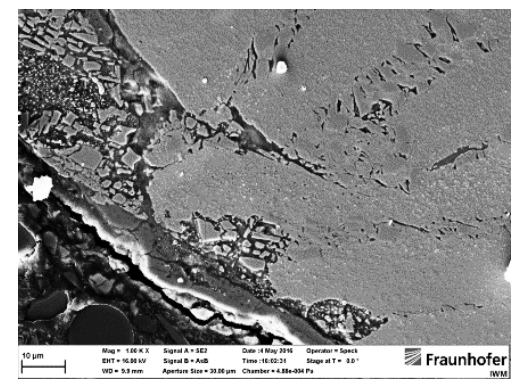

(j)
SM

VS

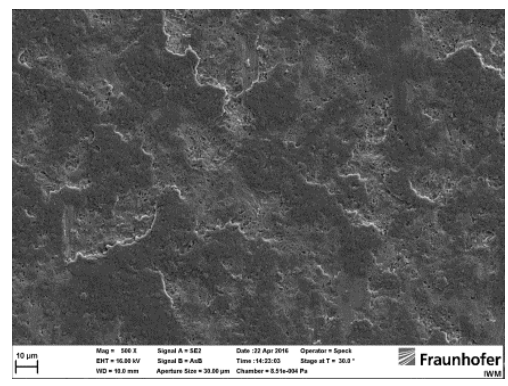

(b)

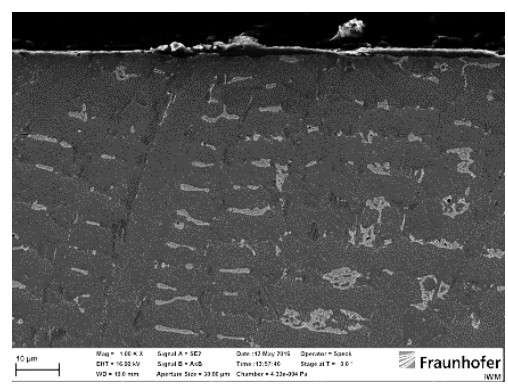

(e)

SR

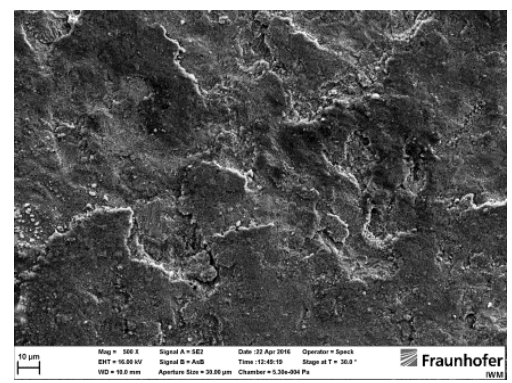

(h)

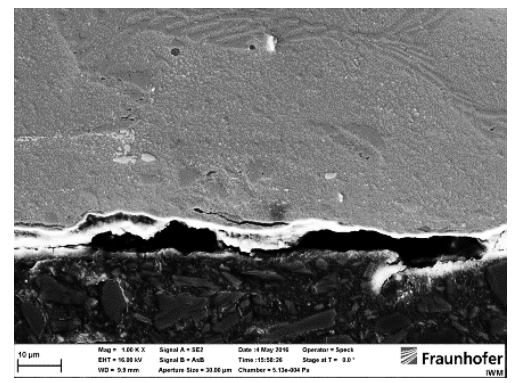

(k)
SAD

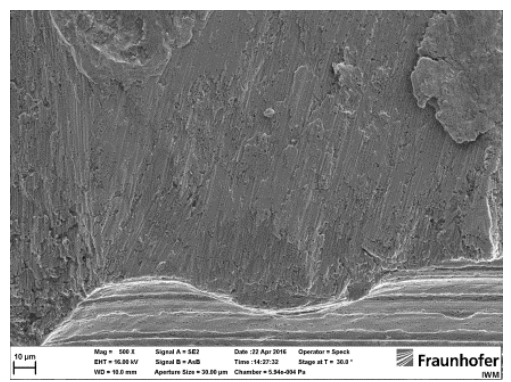

(c)

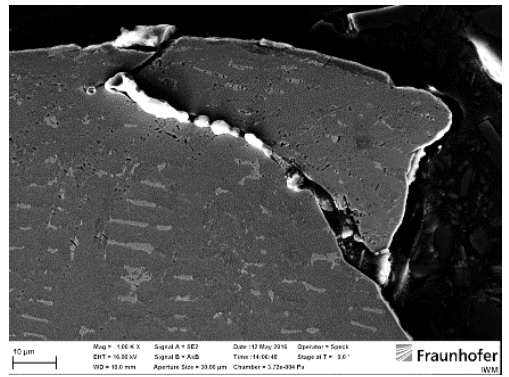

(f)

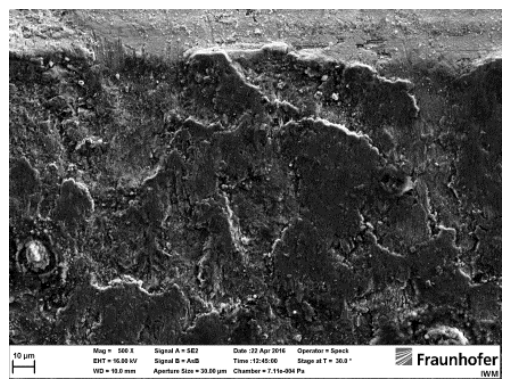

(i)

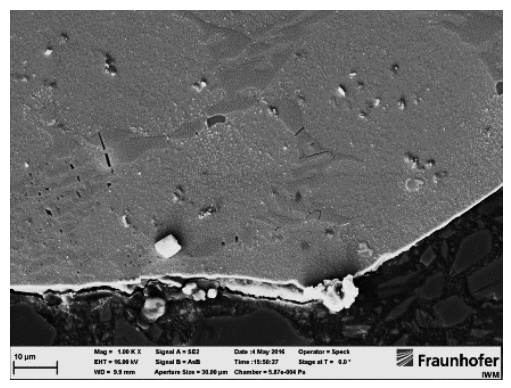

(I)

Abbildung 4.14: REM-Aufnahmen von VS und SR in Draufsicht und im Querschliff unter 220 bar bei $380{ }^{\circ} \mathrm{C}$ an Luft; 500x (a) - (c) und (g) - (i), 1000x (d) - (f) und (j) - (I) 
Wie im Versuch unter 180 bar lokal zu sehen ist, kann in allen Schliffen vom 220 bar Versuch an der Oberfläche eine dunkle Randschicht durchgängig erkannt werden. Bei 1000facher Vergrößerung wirkt diese Schicht strukturlos. In der Detailaufnahme in Abbildung 4.15 bei 5000facher Vergrößerung ist eine ca. 1 bis $2 \mu \mathrm{m}$ dicke kompaktierte Triboschicht zu erkennen. Das EDS-Spektrum 1 detektiert in der Triboschicht eine chemische Zusammensetzung aus Co, $\mathrm{Fe}, \mathrm{Cr}, \mathrm{O}, \mathrm{C}$ und W. Spektrum 2 weist die Elemente $\mathrm{Co}, \mathrm{Cr}$, Fe, C und W nach, wobei die Zone unterhalb der Triboschicht von Spektrum 2 als mechanisch gemischt angesehen werden kann. Die Spektren 1 und 2 weisen zum einen durch den Fe-Anstieg von 12 auf 21 at.-\% auf eine Werkstoffübertragung und zum anderen durch die Messung von $O$ in der Triboschicht auf die Bildung von Oxidationsprodukten an der Kontaktfläche hin. Der Gegenkörper SR präsentiert sich im Vergleich zu den Versuchen unter 140 und 180 bar unterschiedlich. Kompaktierte Schuppen und Ausbrüche in den Bereichen SM und SAD kennzeichnen die Draufsicht. Lokale Vertiefungen in der SM und Risse am SID in den Querschliffen stützen die Beobachtungen der Draufsicht. Daneben sind Risse in den Hartphasen in allen Bereichen der Probe zu sehen. Als Gemeinsamkeit können für den SR über alle drei Laststufen Risse in den karbidischen Hartphasen aus den Querschliffen festgehalten werden. Weiterhin kann die Ausbildung eines Dehnungsgradienten nicht beobachtet werden.

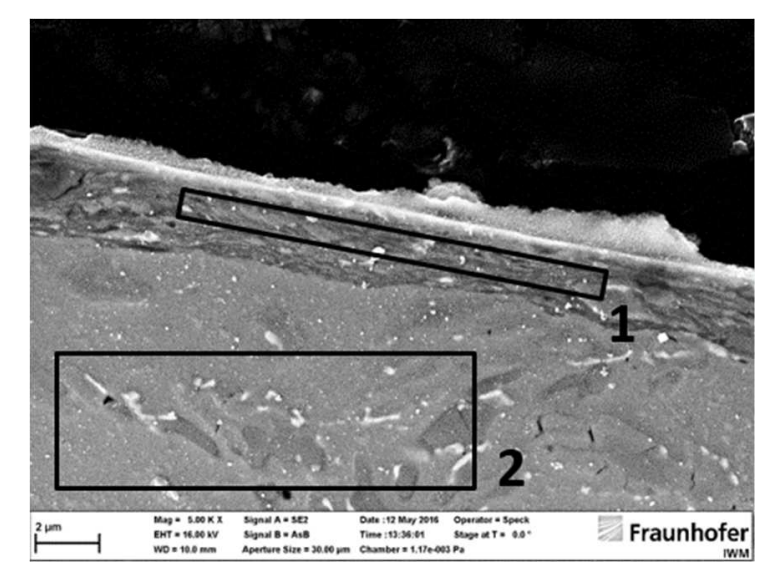

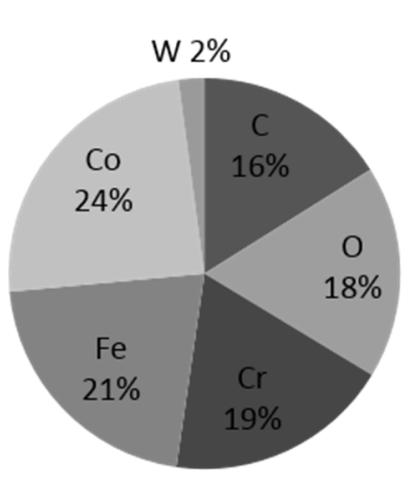

Spektrum 1

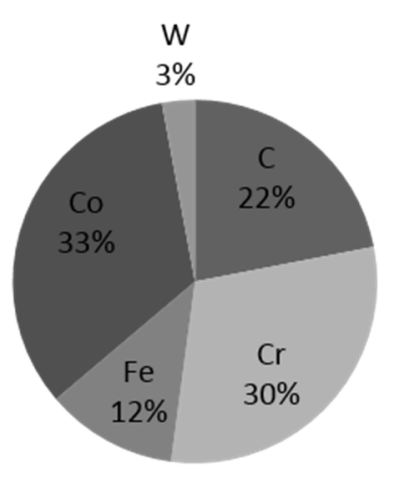

Spektrum 2

Abbildung 4.15: Detailaufnahme aus Abbildung 4.14 (d) mit EDS-Spektren in at.-\% der Triboschicht (1) und mechanisch gemischter Randschicht (2); Vergrößerung 5000x 


\section{Diskussion}

Im Kapitel Diskussion sollen ausgewählte Resultate der vorliegenden Dissertation herausgegriffen und mit Ergebnissen anderer Untersuchungen diskutiert werden. Im ersten Abschnitt wird die Validierung des neu entwickelten VVPs und im zweiten der mikrostrukturelle Einfluss besprochen. Im dritten und vierten Abschnitt sollen neue Erkenntnisse aus den ersten Prüfstandsversuchen zum Einfluss der beiden Hauptparameter Ventilschließen und Verbrennungsdruck auf den Ventilverschleiß erläutert werden, um im letzten Abschnitt Schlussfolgerungen für zukünftige Großgasmotoren zu nennen.

\subsection{Validierung des VVPs}

In der wissenschaftlichen Literatur wird von sieben VVPen auf Komponentenebene berichtet [52], [138], [139], [143], [158], [159] und [168]. Davon betrachten fünf die Applikation Automotive [52], [139], [143], [158], [159] und zwei die Applikation Heavy-Duty [138], [168]. Ein VVP im Segment Großmotor ist bislang nur aus einer Patentschrift bekannt [167]. Somit ist der in dieser Arbeit vorgestellte VVP eine erste Neuentwicklung für die Applikation Großgasmotor.

Als Kriterien zur Prüfstandsvalidierung wurden in den bisherigen Arbeiten die Kenngrößen Verschleißerscheinungsformen, Verschleißtiefe, Verschleißmarkenbreite und Rauheit herangezogen, wobei sich je drei Arbeiten auf einen Vergleich mit dem befeuerten Motorbetrieb oder literaturbekannten Verschleißerscheinungen bezogen haben. Eine Arbeit berichtet von keiner Validierung [159]. In Tabelle 5.1 ist eine Übersicht der Validierungskriterien den bekannten VVPe wiedergegeben.

Tabelle 5.1: Übersicht zur Validierung bekannter VVPe auf Komponentenebene

\begin{tabular}{|c|c|c|c|c|c|c|}
\hline \multicolumn{2}{|c}{ Nr. Arbeit } & $\begin{array}{c}\text { Verschleiß- } \\
\text { erscheinungs- } \\
\text { formen }\end{array}$ & $\begin{array}{c}\text { Verschleiß- } \\
\text { tiefe }\end{array}$ & $\begin{array}{c}\text { Verschleiß- } \\
\text { marken- } \\
\text { breite }\end{array}$ & Rauheit & $\begin{array}{c}\text { Befeuerter } \\
\text { Motorbetrieb }\end{array}$ \\
\hline 1 & {$[52]$} & $x$ & - & - & - & $x$ \\
\hline 2 & {$[138]$} & $x$ & $x$ & - & - & $x$ \\
\hline 3 & {$[143]$} & - & $x$ & - & - & - \\
\hline 4 & {$[139]$} & - & - & $x$ & $x$ & - \\
\hline 5 & {$[158]$} & - & - & $x$ & $x$ & - \\
\hline 6 & {$[159]$} & - & - & - & - & - \\
\hline 7 & {$[168]$} & $x$ & - & $x$ & $x$ & $x$ \\
\hline
\end{tabular}


Alle vier Hauptverschleißmechanismen wurden nach Quellenlage für den Ventilverschleiß identifiziert, wobei im Detail nicht vollständig verstanden ist, welche Mechanismen eigenschaftsbestimmend wirken [43]. Den bisherigen Arbeiten ist keine Zuordnung der Verschleißanteile für die Ventilphasen Schließen und Verbrennungsdruck am Gesamtventilverschleiß zu entnehmen. Inwieweit eine Validierung von VVPen anhand von Literaturvergleichen zulässig ist, wo eine genaue Kenntnis der Belastungskollektive fehlt, erscheint fraglich. Daher sind Schlussfolgerungen aus den Arbeiten [139], [143] und [158] kritisch zu sehen. Darüber hinaus sind Schlussfolgerungen aus Arbeiten mit nicht validierten VVPen wie [159] fragwürdig. Als Fazit erscheint ein Vergleich von VSn aus Prüfstands- und Motorversuch aufgrund des multifaktoriellen Einflusses für eine Validierung notwendig, um die eigenschaftsbestimmenden Verschleißmechanismen zu identifizieren und somit an gezielten Lösungsansätzen zu arbeiten. Im nächsten Schritt gilt es die Frage zu beantworten, welches Kriterium eignet sich für einen derartigen Vergleich? Aus [148] und [155] ist im Detail bekannt, dass die elastische Durchbiegung des Ventiltellers infolge Verbrennungsdruck mit steigendem Druck bei gleichbleibender Tellerdicke ansteigt. Die Verschleißmarkenbreite ist ein zwangsläufiges Resultat der Tellerdurchbiegung. Ferner beeinflussen fertigungsbedingte Toleranzen insbesondere beim Sitzwinkel die Ergebnisse der Verschleißmarkenbreite. Daher ist diese Kenngröße eingeschränkt geeignet, wenn Verbrennungsdruck und Temperatur nicht im Detail bekannt sind. Rauheit und Verschleißtiefe werden von der tribologischen Beanspruchung bestimmt und können Momentaufnahmen sein. In [156] wurden zwei differierende Triboschichten auf VSn des gleichen Motortyps unter verschiedenen Lastkollektiven analysiert. Die eine Schicht war glatt und bestand großteils aus Verbrennungsrückstanden, die andere war aufgeraut und setzte sich aus metallischen Oxiden zusammen. Die Verschleißverhalten beider VS bewirkten keinen vorzeitigen Funktionsausfall. Zur Verschleißtiefe, wie Sin et al. 1979 in [189] untersuchten, können eingebettete Verschleißpartikel zu einer Furchung führen und somit die Rauheit beeinflussen. Verlassen derartige Partikel das Tribosystem, kann zeitlich begrenzt eine andere Verschleißtiefe resultieren. Somit sind Rauheit und Verschleißtiefe keine geeigneten Kriterien für eine Prüfstandsvalidierung. Verschleißerscheinungsformen beschreiben verschleißinduzierte Veränderungen der Oberfläche und Mikrostruktur [14]. Die Untersuchungen von Renz et al. 2017 in [60] an VSn aus demselben Typ Großgasmotor verdeutlichen andere Verschleißerscheinungsformen für Stellite ${ }^{\mathrm{TM}} 12$ im Vergleich zu Tribaloy ${ }^{\mathrm{TM}}$ T400 unter gleichem Lastkollektiv. Nach Fischer 1996 in [190] wird das Verschleißverhalten von tribotechnischen Werkstoffen von der Mikrostruktur und den Eigenschaften der Phasen bestimmt. Aus den genannten Erkenntnissen erscheint es daher sinnvoll, Verschleißerscheinungen nicht losgelöst, sondern im Kontext von Belastungskollektiv und Werkstoff zu bewerten. Somit können Verschleißerscheinungsformen ein geeignetes Kriterium sein. Wenn die Erscheinungen im Verbund mit tribotechnischen Werkstoffen und einer quantitativen Kenngröße wie der Verschleißrate gestellt werden, ist eine vielversprechende Validierung und damit eine Ergebnisübertragbarkeit auf den befeuerten Motorversuch gerechtfertigt. 
Für die Validierung wurden in dieser Arbeit vier Kriterien untersucht; die drei quantitativen Kriterien Ventilrotation, Kühlsystem und Verschleißrate sowie das qualitative Kriterium Verschleißerscheinungsform. Ventilrotation, Kühlsystem und Verschleißrate wurden bislang in keiner Arbeit vorgestellt. Betrachtet man die unterschiedlichen Morphologien der Verschleißerscheinungen, die in den Versuchsreihen zum Ventilschließen und Verbrennungsdruck beobachtet wurden, ist eine ausschließliche Validierung auf Basis qualitativer Kriterien wie Verschleißerscheinungen kritisch zu sehen. Draufsichten und Querschliffe sind immer Momentaufnahmen. Die Phänomene schwerer und milder Verschleiß können hierüber nicht vollständig erklärt werden.

Kühlsystem und Ventilrotation. Werkstoffeigenschaften sind temperaturabhängig. Die Temperatur von VS und SR wird entscheidend von der Kühlung und Ventilrotation mitbestimmt [110]. Aus diesem Grund wurden Kühlung und Ventilrotation in dieser Arbeit validiert. Mit Verwendung der originalen Ventildrehvorrichtung im Prüfmodus I wurde eine bereits vielfach erprobte Lösung eingesetzt. Im Prüfmdodus II musste aufgrund des reduzierten Ventilhubs eine servohydraulisch-mechanische Ersatzdrehvorrichtung entwickelt werden. Mittels eines Kraftaufnehmers wurde die Ventilrotation indirekt validiert. Für den Fall einer Fehlfunktion hätte ein Drehmoment gemessen werden müssen. Dies war nicht der Fall. Darüber hinaus konnte man ganz pragmatisch über das Sichtfenster der Klimakammer die Ventilrotation visuell prüfen. Zur Validierung der Kühlung wurden je drei Messungen bei 330, 380 und $430{ }^{\circ} \mathrm{C}$ in jedem Prüfmodus durchgeführt. Der VVP verfügt über drei Kühlkreisläufe, somit konnten die vorgegebenen Temperaturen und Deltas zwischen VS und SR erreicht werden. Da eine Validierung der Kriterien Ventilrotation und Kühlsystem für die literaturbekannten VVPe nicht beschrieben wurde, sind deren Versuchsergebnisse und Schlußfolgerungen kritisch zu betrachten.

Verschleißerscheinungsformen. Im Vergleich der untersuchten Proben aus befeuertem Motorbetrieb (s. Kapitel 2.3) und Prüfstandsversuch konnten für denselben tribotechnischen Werkstoff Stellite ${ }^{\mathrm{TM}} 12$ die gleichen charakteristischen Veränderungen in der tribologisch beanspruchten Randschicht beobachtet werden. Darüber hinaus waren die Art und Form der entstehenden Verschleißpartikel vergleichbar. Die typischen Verschleißerscheinungen des Motorversuchs mit Schuppen, Kuppen und Mulden an der Oberfläche sowie zerbrochenen metallkeramischen Hartphasen im Dehnungsgradienten oder parallelen Rissen zur Oberfläche konnten gleichfalls in den Aufnahmen aus den Versuchreihen zum Ventilschließen und Verbrennungsdruck nachgewiesen werden. Somit ist die Schlussfolgerung einer erfolgreichen Validierung in Bezug auf das Kriterium Verschleißerscheinungsformen zulässig.

Verschleißrate. Als quantitative Kenngröße wurde in dieser Arbeit die Verschleißrate in Volumen pro Zeit eingeführt. Eine solche Kenngröße wurde in den bisherigen Arbeiten zum Ventilverschleiß nicht angewendet. Für die VSn aus den befeuerten Motorversuchen wurden im Kapitel 2.3 Verschleißraten von bis zu 3,95 und 11,81 mm³/h für EVS bzw. AVS gemessen. Es ist anzumerken, dass die ermittelten Verschleißraten der Motorversuche aus den kombinierten Ventilphasen Schließen und Verbrennungsdruck resultieren. Ein direkter Vergleich der Verschleißraten aus Motorbetrieb und experimenteller Simulation ist daher begrenzt zulässig. Als vielmehr geeignete Betrachtungsweise der experimentell ermittelten 
Verschleißraten empfiehlt es sich daher, die Ergebnisse als Trend zu verstehen. Die größten Verschleißraten aus den Versuchsreihen zum Ventilschließen und Verbrennungsdruck betragen 1,97 bzw. 2,28 mm³ $/ \mathrm{h}$. Diese Werte liegen in derselben Größenordnung, wie die der untersuchten VSn aus dem Motorversuch. Die Schlussfolgerung einer erfolgreichen Validierung für das Kriterium Verschleißrate ist somit erfüllt.

Mit den Ergebnissen der Validierung konnte nachgewiesen werden, dass der neu entwickelte VVP die eigenschaftsbestimmenden Verschleißmechanismen, wie sie aus den Untersuchungen an VSen aus dem befeuerten Motorbetrieb identifiziert wurden (vgl. Kapitel 2.3), nachbilden kann. Durch eine getrennte Simulation der Ventilphasen Schließen und Verbrennungsdruck bietet der VVP als neues Werkzeug die Möglichkeit, die dominierenden Verschleißmechanismen den Ventilphasen Schließen und Verbrennungsdruck qualitativ wie quantitativ zu zuordnen. Die durchgeführten Versuchsreihen und der Dauertest über $447 \mathrm{~h}$ belegen, dass der Prüfstand über Millionen Lastzyklen mit einer kontrollierten Parametervariation betrieben werden kann. Erste Ergebnisse unterstreichen die Fähigkeit des Prüfstands, Einflüsse einzelner Parameter auf das Verschleißverhalten des Tribosystem VS/SR zu quantifizieren. Beliebig häufige Versuchsunterbrechungen und intervallmäßige Verschleißprofilmessungen eröffnen die Möglichkeit für ein besseres Verständnis des Verschleißverhaltens über die Zeit. Veränderungen der Verschleißrate und mögliche Mechanismenwechsel können somit verfolgt werden. Darüber hinaus kann mit Hilfe der Parameterüberwachung eine Verlinkung von speziellen Verschleißsituationen mit Einzelereignissen hergestellt werden. Die dadurch zusätzlich generierten Informationen können im Vergleich zum kumulierten Verschleiß von VSn aus dem Feld oder Motorenprüfstandstests, neue wie auch ausschlaggebende Erkenntnisse zum Ventilverschleiß beitragen. Die Wahl eines steifen Prüfrahmens hat sich bewährt, um den Einfluss von Elastizitäten auf das Prüfergebnis zu minimieren. Darüber hinaus schafft dieser die Möglichkeit zu einem späteren Zeitpunkt in Verbindung mit dem integrierten Kraftaufnehmer schließgeschwindigkeits- oder verbrennungsdruckabhängige Reibleistungen zu messen. In Bezug auf die Temperatur wurden unterschiedliche Verschleißerscheinungsformen bei RT und $380^{\circ} \mathrm{C}$ festgestellt. Neben kleineren Verschleißmarkenbreiten in der Draufsicht konnten keine Schädigungshinweise in den Querschliffen bei RT unter beiden Belastungsfällen beobachtet werden. Hingegen wurde bei $330{ }^{\circ} \mathrm{C}$ ein deutlicher Dehnungsgradient in der tribologisch beanspruchten Randschicht unter $0,6 \mathrm{~m} / \mathrm{s}$ und eine kompaktierte Triboschicht unter 180 bar gefunden. Im Ergebnis konnte somit eine temperaturinduzierte Veränderung in der beanspruchten Randschicht nachgewiesen werden. Zusammenfassend wurde ein neuartiger VVP für Großgasmotoren entwickelt, der eine vielversprechende Basis für die Generierung neuer Erkenntnisse schafft und somit für ein verbessertes Verständnis über das tribologische Verhalten des Systems VS/SR in Großgasmotoren beitragen wird. 


\subsection{Einfluss der Mikrostruktur}

Im Kapitel 2.3 wurden Proben aus befeuerten Motorversuchen desselben Typs Großgasmotor mikrostrukturell charakterisiert. Die ermittelten Verschleißraten reichten von 1,45 bis $11,81 \mathrm{~mm}^{3} / \mathrm{h}$. Aufgrund der großen Verschleißraten und dem vorzeitigen Bauteilversagen aller Proben kann eine Einlaufphase ausgeschlossen werden. Vielmehr scheint es, dass das Tribosystem VS/SR ein lineares Verschleißverhalten mit großer Verschleißrate von Anfang an aufwies. Wie kannn dieses Verschleißverhalten erklärt werden?

Dimensionierung, Oberflächentopographie wie Rauheit und Welligkeit sowie die verwendeten tribotechnischen Werkstoffe können als gleich für alle 4 Proben angenommen werden, da die Komponenten aus derselben Fertigung und seriellen Planproduktionsaufträgen stammen. Es sind keine Prototypenteile. Der Gegenkörper SR war in allen Fällen geometrisch und werkstofftechnisch gleich und stammte gleichfalls aus serieller Produktion, jedoch einer anderen als die vier VSn. Nähere Angaben zum SR können aufgrund Fremdfabrikat nicht gemacht werden. Bei gleicher Dimensionierung und Fertigungstechnologie insbesondere des Differenzwinkels zwischen VS und SR darf von einer ähnlichen Kontaktmechanik zu Beginn ausgegangen werden. Die Kontaktsituation beginnt am SAD als Linienkontakt und entwickelt sich über die Zeit mit zunehmender Verschleißmarkenbreite zum Vollkontakt. Die Mikrostrukturanalyse verdeutlicht in den Querschliffen anfangs nach $3 \mathrm{~h}$ erste Versetzungen und Scherbänder in den metallischen Zellen (Abbildung 2.10 (a)), die sich mit anhaltender Betriebsdauer zu einem mehreren Zehntelmikrometer tiefgehenden Dehnungsgradienten mit zerbrochenen Hartphasen aufakkumulieren (Abbildung 2.9 (b)). Im weiteren Verlauf kommt es zur Ausbildung einer plastisch formgeänderten, korngefeinten Randschicht (Abbildung 2.10 (b)). Dieses Phänomen kann aus den wirkenden plastischen Dehnungen erklärt werden. In den primär erstarrenden Co-Cr-Mischkristallzellen des Kompositwerkstoffs Stellite ${ }^{\mathrm{TM}} 12$ sind die metallkeramischen Hartphasen aus $\mathrm{Cr}$ - und W-Karbiden in dendritischer Struktur eingebettet. Aufgrund seiner Kristallstruktur verfügt der Co-Cr-Mischkristall über mehrere planare Gleitebenen, wodurch eine Vielzahl an Versetzungen aufakkumuliert werden kann. Hingegen sich die Gleitebenen der Hartphasen blockiert. Somit können sich unter anhaltend zyklischer Belastung adiabatische Scherbänder in Form von Dehnungsgradienten ausbilden, in welchen ein Zerbrechen der metallkeramischen Hartphasen zu beobachten ist. Der mechanischdominierte Verschleißmechanismus Adhäsion bestimmt die Verschleißvorgänge. Im weiteren Verlauf kommt es in der oberflächennahen Randschicht zu einem mechanischen Mischen der feinzerbrochenen Hartphasenpartikel mit den metallischen Zellen, wobei oberflächliche Partikel in tiefere Zonen eingetragen werden können. Daneben finden sich in der tribologisch beanspruchten Randschicht die Verschleißerscheinungsformen parallele Rissen zur Oberfläche (Abbildung 2.9 (d)) und schuppenförmige Verschleißpartikel, wie sie typisch für den Verschleißmechanismus Oberflächenzerrüttung mit dem Untermechanismus Delamination sind.

Zusammenfassend können somit die zwei mechanisch-dominierten Verschleißmechanismen Adhäsion und Oberflächenzerrüttung mit dem Untermechanismus Delamination für den tribotechnischen Werkstoff Stellite ${ }^{\mathrm{TM}} 12 \mathrm{im}$ Tribosystem VS/SR in Großgasmotoren festgestellt 
werden. Die untereutektisch-induzierte Mikrostruktur mit typischer Formgröße der karbidischen Hartphasen und dendritischer Struktur in großen Co-Cr-Mischkristallzellen begünstigt die Bildung von Dehnungsgradienten, die sich unter anhaltend zyklischer Belastung in der oberflächennahen Randschicht in eine mechanisch gemischte Randschicht verändert. Überlagert werden diese Verschleißvorgänge durch Tribooxidation und Temperatur. Ein zunehmendes Eindringen von Sauerstoff über die Zeit, wie es die XPS-Ergebnisse erkennen lassen, bewirkt eine Zunahme der Oxidationsstufen in der tribologisch beanspruchten Randschicht. Woodford und Bricknell konnten 1981 in [182] eine Versprödung an den Korngrenzen von Nickelbasislegierungen infolge Sauerstoffpenetration nachweisen. Beschichtungen, welche ein Eindringen von Sauerstoff verhinderten, konnten einer Versprödung vermeiden, berichten die Autoren. Daher wird angenommen, dass ein zunehmendes Eindringen von Sauerstoff in den tribotechnischen Werkstoff Stellite ${ }^{\mathrm{TM}} 12 \mathrm{zu}$ einer Veränderung der Haftfestigkeit an der Grenzfläche zwischen karbidischer Hartphase und Metallmatrix führen könnte. Ferner ist aufgrund der höchsten Verschleißrate von Probe A4 davon auszugehen, dass die Geschwindigkeit der Sauerstoffpenetration mit steigender Temperatur zunimmt und die Warmfestigkeit des tribotechnischen Werkstoffs abnimmt, sodass in der Folge der Verschleiß steigt. Vergleicht man die maximalen Verschleißraten aus der experimenteller Simulation zum Ventilschließen und Verbrennungsdruck mit 2,71 bzw. $2,36 \mathrm{~mm}^{3} / \mathrm{h}$, scheint der tribotechnische Werkstoff Stellite ${ }^{\mathrm{Tm}} 12$ gleichermaßen Stoß- und Gleitverschleißbeanspruchungen zu widerstehen.

\subsection{Neue Erkenntnisse zum Einfluss des Ventilschließens}

Nach Quellenlage wird das Ventilschließen als wichtiger [52], [138], [143], [159] oder wichtigster [137] Faktor angesehen. Eine anteilige Zuordnung des Ventilschließverschleißes am Gesamtventilverschleiß bleibt bislang in der wissenschaftlichen Literatur unbeantwortet. Darüber hinaus ist offen, wie sich der Komponentenverschleiß mit steigender Schließgeschwindigkeit entwickelt. Die ersten Ergebnisse zur experimentellen Simulation des Ventilschließens bestätigen die Schließgeschwindigkeit als signifikanten Einflussfaktor auf das Verschleißverhalten des Tribosystems VS/SR. Bei konstant gleicher Temperatur von $380^{\circ} \mathrm{C}$ war ein Anstieg der Komponentenverschleißraten im untersuchten Bereich von 0,2 bis $1,0 \mathrm{~m} / \mathrm{s}$ näherungsweise um die Faktoren 4 und 10 für VS bzw. SR zu beobachten, somit darf als neue Erkenntnis eine größere Zunahme des Komponentenverschleißes SR am Systemverschleiß festgestellt werden (Abbildung 4.3). Die in Relation 2,5fach höhere Zunahme des SRVerschleißes zur VS kann aus den verwendeten Werkstoffen erklärt werden. Die CoBasislegierung Stellite ${ }^{\mathrm{TM}} 12 \mathrm{im}$ Sitzbereich der VS besitzt im Vergleich zum SR-Werkstoff Pleuco $12 \mathrm{MV}$, welcher der Gruppe der Kaltarbeitsstähle zu zuordnen ist, eine höhere Schlagbeständigkeit. Der Aspekt der unterschiedlichen Werkstoffe erklärt vermutlich die Komponentenverschleißbeträge am Systemverschleiß unter steigender Last. Jedoch hat der Anstieg der Verschleißraten von Grund- und Gegenkörper noch weitere Ursachen. Bei Betrachtung der Verschleißtiefenprofile der VS sind ein fehlender Verschleiß im Bereich des SIDs, ein annähernd lineares Verschleißverhalten mit zunehmender Konkavität bei größer 
werdender Schließgeschwindigkeit über die Zeit und eine Profilspitze am Tellerdurchmesser markant. Der erkennbar fehlende Verschleiß am SID von ca. 0,5 mm, welcher unter Verbrennungsdruck nicht vorhanden ist, kann zwei Gründe haben. Die Aufschlagskräfte sind mit bis zu ca. $8 \mathrm{kN}$ bei maximaler Schließgeschwindigkeit von $1,0 \mathrm{~m} / \mathrm{s}$ im Vergleich zur Lasteinleitung unter Verbrennungsdruck mit 80 bis 130 kN um eine Größenordnung kleiner, woraus eine kleinere elastische Tellerdurchbiegung resultiert. Daneben führt die Dimensionierung mit ungleichen Teilkreisen der SID von VS und SR mit 72,84 bzw. 74,0 mm in Verbindung mit dem Differenzwinkel zu keinem Kontakt in diesem Bereich. Der zunehmend konkave Tiefenprofilverlauf unter steigender Last über die Zeit resultiert vermutlich aus StickSlip-Effekten am Ventilsitz. Stick-Slip-Effekte werden im Reibkontakt von der Kontaktsteifheit von Körpern bestimmt [191]. Die Tellerhöhe am SID ist mit 10,6 mm 3,8 mm dicker als am SAD, somit stellt sich am SAD im Vergleich zum SID eine kleinere Steifigkeit ein. Im Kontakt führt dies zu zunehmenden Gleiten am SAD und Anhaften am SID. Die Draufsichten unter $1,0 \mathrm{~m} / \mathrm{s}$ in den Bereichen SM und SAD stützen diese These mit typischen Plateaus wie sie unter Gleitreibung auftreten. Hingegen erscheint der Bereich SID schuppiger und deutlich topographiert, wie es eher typisch für ein Anhaften ist. Nimmt man zunehmendes Gleiten im Bereich des SAD an, so führt das Mikrogleiten zu plastischen Dehnungen. Lokal kann über die Zeitachse ein unterschiedlicher Verschleiß resultieren, der sich im Ergebnis als konkaves Verschleißprofil darstellt. Addierend wirkt in diesem Kontext die Kontaktsituation zwischen VS und SR auf die Verschleißrate. Konstruktiv ist der Kontakt auf Außentrag mit einem nominellen Differenzwinkel von 12` ausgelegt. Im Moment des Erstkontakts herrscht folglich Linienkontakt (vgl. Abbildung 2.5 (a)). Vergleichsweise wenige Asperiten bilden die Anzahl der Mikrokontakte. Nach Bowden und Tabor [33], [35] kann es an diesen Kontakten zu plastischen Dehnungen kommen. Sodass sich eine überwiegend elastische oder plastische Kontaktsituation einstellen kann. Die anhaltende Belastung bewirkt im weiteren Verlauf die Entstehung von Verschleißpartikeln. Diese können nach Berthier [108] vollständig oder teilweise im Reibspalt als dritter Körper verbleiben. Für den Fall, dass die Partikel im Reibspalt verbleiben, bestimmen Art und Beschaffenheit des Verschleißpartikels die entstehenden Folgen und damit den Verschleiß. Es kann zur Bildung einer kompaktierten Triboschicht kommen (Abbildung 4.4 (d) bis (f)), die Partikel können eingedrückt und in der Randzone mechanisch gemischt werden. Bei ausreichend größer Härte hingegen könnte es zu abrasivem Verschleiß kommen [190], der nicht an den Proben in dieser Arbeit beobachtet wurde. Darüber hinaus können die mikrometergoßen Partikel, wie diese in Abbildung 4.6 (d) bis (f) zu sehen sind, eine nicht gleichmäßige Kontaktsituation mit Punkt- oder Linienkontakten bewirken. Mit Verschleiß kann sich die Kontaktsituation ändern, sodass die Kontaktart von überwiegend elastisch zu plastisch wechseln kann. Ein mechanisch-dominierter Verschleißmechanismus kann in Kombination mit Stick-Slip-Effekten lokal höheren Verschleiß bewirken. Die Tiefenprofilspitzen am SAD der VS resultieren aus der Kontaktmechanik von VS und SR. Der Linienkontakt im Bereich des SADs ergibt sich aus der Kombination von unterschiedlichen Teilkreisen der SAD von VS und SR mit 86,0 bzw. 85,9 mm mit einem Ventilüberstand von $0,05 \mathrm{~mm}$ sowie Differenzwinkel. Diese konstruktivbedingte Kontaktsituation erklärt das beobachtete Phänomen aufgeschobener Schuppen am SID. 
Kommen VS und SR im Neuzustand in Kontakt herrscht Außenkontakt. Es bildet sich ein keilförmiger Reibspalt. Somit ergeben sich für die Verschleißpartikel zwei Möglichkeiten das System zu verlassen. Zum einen können sie beim Entkoppeln von VS und SR herausfallen oder aber sie werden aus dem Reibspalt in Richtung SID herausgeschoben.

Unter Last kommt es zu einem oszillierendem Mikrogleiten zwischen den Kontaktflächen von VS und SR. Im Fall der Lastzunahme kann der Werkstoff in Richtung des SADs plastisch gedehnt werden, hingegen ist eine plastische Dehnung im Fall der Lastabnahme nicht anzunehmen. Daher kann als Ursache der plastischen Deformationen am SID und SAD „herausgedrückter" Werkstoff aus dem Reibspalt vermutet werden.

Der nominelle Kontakt, der aus einer Vielzahl von im Kontakt stehenden Asperiten besteht, bestimmt durch seine Oberflächentopogrphie die Energiedissipation. Anzahl der Asperiten und deren Höhe können konstant sein, doch die Lokalisierung von Bergen und Tälern innerhalb der Topografie verändert sich im Kontakt ständig. Da die Energiedissipation über die Asperiten erfolgt und diese zufällig lokalisiert sind, ist der Prozess stochastisch im Hinblick auf Ort und Zeit. Ein mechanisches Mischen erfolgt somit nicht gleichzeitig überall und der Eintrag an Aktivieriungsenergie ist an den Orten höchster Flächenpressung am größten. In der Folge bilden sich Stick-Slip-Effekte lokal unterschiedlich aus, die durch die radiusabhängige Steifigkeit des Ventiltellers überlagert werden. Es wird angenommen, dass ein zunehmendes Gleiten im Bereich des SADs aufgrund der kleineren Steifigkeit im Vergleich zum SID auftritt. Dadurch kann die Kontaktfläche der VS lokal unterschiedlich verschleißen. Die gemessenen Verschleißprofiltiefen mit zunehmender Konkavität am SAD der VS und Konvexität an SR stützen dieses. Die Systemverschleißrate nimmt in etwa um den Faktor 4,7 von 0,2 auf 1,0 m/s für die untersuchten Schließgeschwindigkeiten zu.

\subsection{Neue Erkenntnisse zum Einfluss des Verbrennungsdrucks}

Neben dem Ventilschließen wird nach Quellenlage der Parameter Verbrennungsdruck als wichtiger [43], [146] oder wichtigster Faktor [144], [145] betrachtet. Es ist bekannt, dass der Verschleiß unter steigendem Verbrennungsdruck zunimmt und bei steigenden Temperaturen abnimmt. Nicht untersucht ist, ob der Verschleiß bei Anstieg beider Parameter Druck und Temperatur zunimmt. Ferner finden sich keine Ergebnisse zum Komponentenverschleiß bei steigendem Druck. Die ersten ermittelten Verschleißprofile und Verschleißraten aus der experimentellen Simulation zum Verbrennungsdruck sind im Vergleich zum Ventilschließen durch ein anderes neues Charakteristikum gekennzeichnet. Mit steigendem Verbrennungsdruck und bei konstant gleicher Temperatur von $380^{\circ} \mathrm{C}$ konnte eine Abnahme der Verschleißrate im untersuchten Bereich von 140 bis 180 bar um annähernd $30 \%$ für die VS ermittelt werden. Der Verschleiß vom SR blieb in etwa gleich. Somit lässt sich der um ca. $20 \%$ reduzierte Systemverschleiß ausschließlich auf die VS zurückführen. Diese neue Erkenntnis steht im Widerspruch zu [147]. Wenn bei steigendem Verbrennungsdruck und konstanter Temperatur die Verschleißrate sinkt, ist anzunehmen, dass der mechanischdominierte Verschleiß um tribochemisch-dominierte Prozesse erweitert wird. Ein vergleichbar 
großer Dehnungsgradient wie unter Ventilschließen ist unter Verbrennungsdruck nicht zu beobachten, vielmehr charakterisieren die beiden Phänomene Triboschicht und mechanisch gemischte Randschicht die oberflächennahe Randzone (Abbildung 4.15). Verschleißinduzierte Partikel von Grund- und Gegenkörper verbleiben anscheinend länger im Reibspalt und bilden auf und zwischen den Asperiten einen dritten Körper nach Berthier [108]. Die EDSdetektierten Sauerstoff- und Metallgehalte in den Triboschichten unter 140 und 220 bar lassen metallische Oxide aus $\mathrm{Fe}, \mathrm{Cr}$ oder $\mathrm{Co}$ vermuten. Metallische Oxidfilme können im metallischen Kontakt infolge tribochemischer Prozesse nach Quinn [180] sowie Stott und Wood [51] den Verschleiß reduzieren. Wenn Metalloxide unter kleiner und großer Last vorhanden sind und die gemessene Verschleißrate mit höherer Last abnimmt, so darf quantitativ eine höhere Bildungsrate an Metalloxiden aufgrund der Zunahme von tribochemischen Prozessen nach Rigney [67] vermutet werden. Im Vergleich der Versuche unter 140 und 220 bar ist unter 140 bar eine lokal begrenzte und unter 220 bar eine geschlossene Triboschicht zu beobachten. Höhere Flächenpressungen unter 220 bar können einen Einfluss auf Haftfestigkeit zwischen Tribomaterial und Verschleißkörper sowie Kompaktierungsgrad der Triboschicht haben. Dicke, Kompaktierungsgrad und Geschlossenheit der Triboschichten scheinen belastungsabhängig zu variieren und somit eine mögliche Ursache für die unterschiedlichen Verschleißraten zu sein. Ein Vergleich der Draufsichten insbesondere der VS stützt die These zum Einfluss des Verbrennungsdrucks auf die Kompaktierung der Triboschicht, wie die geglätteten Kontaktflächen erkennen lassen.

Im Reibkontakt wird Energie dissipiert. Die umgewandelte Energie kann in der tribologisch beanspruchten Randschicht zu einer veränderten Mikrostruktur [105], wie der beschriebenen mechanisch gemischten Randschicht führen, oder als Eintrag von Aktivierungsenergie wirken. Die ausgelösten chemisch-physikalischen Prozesse können neben Diffusionsvorgängen auch eine erhöhte Oxidationsneigung sein, wie die XPS-Ergebnisse aus Kapitel 2.3.3 schlußfolgern lassen. Betrachtet man unter diesem Aspekt das Tribosystem VS/SR und die ersten Ergebnisse dieser Arbeit, kann folgender Prozess vorgeschlagen werden. Nach den ersten $25 \mathrm{~h}$ ist die Verschleißrate bei allen drei Laststufen ähnlich groß, danach unterscheiden sich diese in Abhängigkeit von der Belastung zunehmend. Beginnend mit plastischen Formänderungen wie Versetzungen, gefolgt von Dehnungsgradienten und zerbrochenen karbidischen Hartphasen kommt es zu einem mechanischen Mischen von zerbrochenen Hartphasenpartikel mit den plastisch deformierten Metallzellen aus Co-Cr-Matrixmaterial in der Randschicht. Im weiteren Verlauf bilden sich erste Verschleißpartikel. Diese Partikel bilden im Verbund mit metalloxidischen Teilchen in Abhängigkeit von der Belastung mehr oder weniger Tribomaterial im Reibspalt über die Zeit. Mit zunehmend induzierter Reibleistung infolge erhöhtem Verbrennungsdruck können die Aktivierungsenergien zunehmen, wodurch vermehrt Hot-Spots auftreten, die nach Quinn [41] eine Oxidation bewirken. Die auftretenden Aktivierungsenergien können eine Oxidation von $\mathrm{Co}$ und $\mathrm{Cr}$ bewirken. Eine Bildung von Cound $\mathrm{Cr}$-Oxiden kann die Reibung zu reduzieren. Parallel wird die sich bildende metalloxidische Triboschicht unter steigendem Verbrennungsdruck kompaktiert, wodurch die Schichtdicke verkleinert und die Anhaftung an der VS bzw. SR verbessert wird. Abbildung 5.1 veranschaulicht schematisch diesen Prozess. Im weiteren Verlauf reduziert die generierte 
Triboschicht die dissipierte Reibleistung in das Tribosystem VS/SR. Die dissipierten Reibenergien werden kleiner. In der Folge minimieren sich mechanisches Mischen und Oxidationsvorgänge. Das Tribosystem kann in den Steady-State wechseln. Bestimmen jedoch mechanisch-dominierte Verschleißmechanismen, wie sie für das Tribosystem VS/SR in dieser Arbeit gefunden wurden, weiterhin die Verschleißvorgänge, ist ein Steady-State über die Zeitachse nicht zu erwarten.

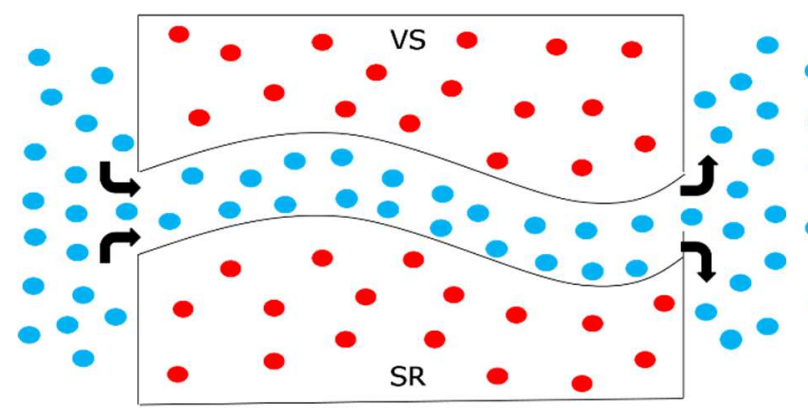

Ausgangszustand

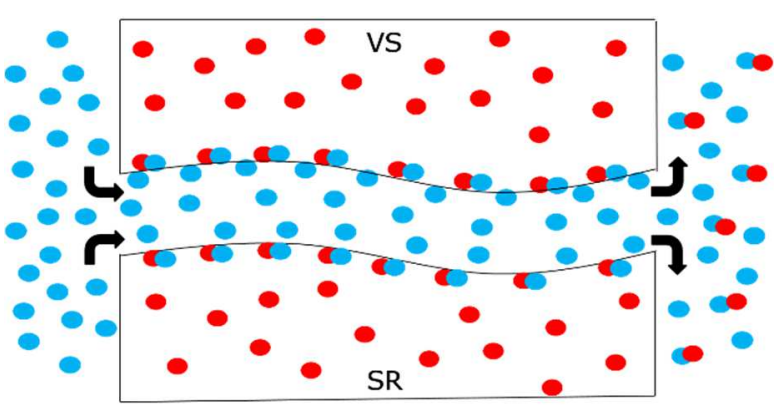

Bildung von Metalloxiden

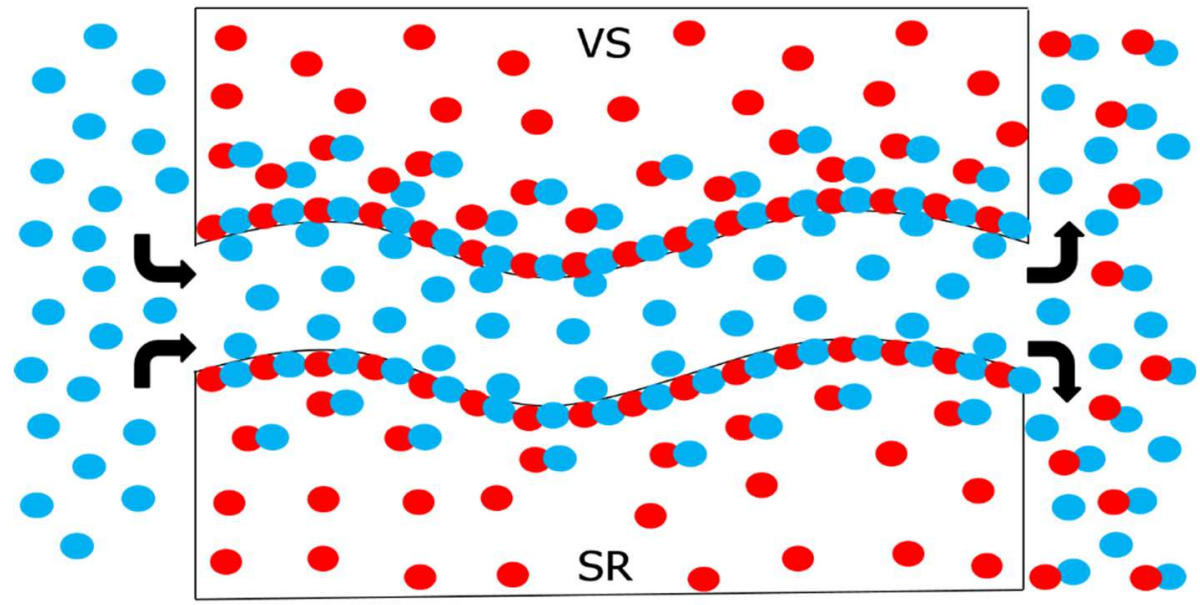

Metalloxidisch angereicherter und kompaktierter Triboschicht

Abbildung 5.1: Rot $=$ Metalle, blau = Sauerstoff, Ausgangszustand (oben links), Bildung von Metalloxiden über die Zeit (oben rechts), metalloxidische Triboschicht (unten)

Die Systemverschleißrate nimmt in etwa um den Faktor 1,3 von 140 auf 220 bar für die untersuchten Verbrennungsdrücke ab. Im Vergleich zu den ermittelten Systemverschleißraten für Ventilschließen resultiert somit eine weitere neue Erkenntnis. Das Tribosystem VS/SR reagiert sensitiver auf den Faktor Ventilschließen. Mit dieser Schlussfolgerung stützt diese Arbeit die These von Fricke und Allen [137] sowie Ootani et al. [141], dass das Ventilschließen im Vergleich zum Verbrennungsdruck der wichtigere Einflussfaktor auf den Ventilverschleiß ist. 


\subsection{Schlussfolgerungen für zukünftige Großgasmotoren}

Aus den ersten Ergebnissen der Prüfstandsversuche geht kein gemeinsam globaler Trend für die Ventilphasen Schließen und Verbrennungsdruck für die untersuchten tribotechnischen Werkstoffe Stellite ${ }^{\mathrm{TM}} 12$ und Pleuco 12 MV hervor.

Verbrennungsdruck. Für zukünftige Großgasmotoren sind Verbrennungsdrücke von bis zu 300 bar zu erwarten. Damit einhergehend werden Flächenpressungen und Mikrogleiten zwischen VS und SR sowie Bauteiltemperaturen zunehmen. Daneben bewirkt eine verschärfende Abgasgesetzgebung eine reduzierte Menge an Verbrennungsrückständen, sodass für die Bildung von verschleißminimierenden Triboschichten auf Basis von Verbrennungsprodukten weniger Edukte zur Verfügung stehen. Im Ergebnis wird die Gleitreibung intensiviert. Da Gleitreibung verglichen mit anderen Reibungsarten die höchsten tribologischen Beanspruchungen im Kontaktbereich bewirkt [14], wie die Ergebnisse aus den experimentellen Simulationen belegen, kommt der Werkstoffauswahl und geometrischen Auslegung von VS und SR eine entscheidende Rolle zu. Untereutektische Legierungen in Verbindung mit bestimmten Eigenschaften der Gefügebestandteile, wie die in dieser Arbeit untersuchte Hartlegierung Stellite ${ }^{\mathrm{TM}} 12$, scheinen nicht die vielversprechenste Wahl, wenn mechanisch-dominierte Verschleißmechanismen vorherrschen. Gleichfalls scheint der SRWerkstoff Pleuco 12 MV keine geeignete Werkstoffwahl zu sein. In diesem Kontext werden die Werkstoffeinflüsse von der mechanischen Kontaktsituation zwischen VS und SR durch Art und Größe des Differenzwinkels und Ventilüberstand überlagert. Bestehende Konstruktionsund Fertigungsansätze sind zu prüfen und zu hinterfragen. Die Möglichkeiten der Finite Elemente Analyse gilt es auszuschöpfen.

Ventilschließen. Zur Erreichung immer höherer Wirkungsgrade spielt die Zylinderfüllung und damit die Schließgeschwindigkeit eine wichtige Rolle. Eine Schließgeschwindigkeit von 0,5 m/s ist Stand der Technik und darf als Kompromiss zwischen Werkstoff und Lebensdauer angesehen werden. Für einen verbesserten Wirkungsgrad wird eine höhere Füllung z.B. durch größere Schließgeschwindigkeiten aus Sicht der Thermodynamik gefordert. Um hier von der Komponentenseite einen Beitrag zu leisten, werden im Sitzbereich Werkstoffe mit hoher Widerstandsfähigkeit gegenüber Stoßbeanspruchung benötigt. Aus den ersten Ergebnissen scheint der Tribowerkstoff Stellite ${ }^{\mathrm{TM}} 12 \mathrm{im}$ Vergleich zum Pleuco $12 \mathrm{MV}$ die bessere Werkstoffwahl zu sein. Dennoch verdeutlichen die betragsmäßig gleichen Verschleißraten von Stellite ${ }^{\mathrm{TM}} 12$ für Schließen und Verbrennungsdruck Optimierungspotenzial.

Als Schlussfolgerungen dürfen festgehalten werden, dass Werkstoffansätze auf Basis untereutektischer Hartlegierungen wie Stellite ${ }^{\mathrm{TM}} 12$ oder Stähle wie Pleuco $12 \mathrm{MV}$ ein eingeschränktes Zukunftspotenzial in Großgasmotoren bieten. Vielmehr werden Werkstoffansätze benötigt, die selbstbildende Triboschichten zur Reibungsminimierung und Stoßdämpfung generieren können. Parallel ist die Kontaktsituation zwischen VS und SR im Detail mittels Finite Elemente Analyse zu untersuchen, ob durch geänderte Ansätze die anfänglich hohen Flächenpressungen verringert werden können. 


\section{Zusammenfassung und Ausblick}

Die vorliegende Arbeit betrachtet die Entwicklung, Konstruktion und den Aufbau eines neuartigen VVPs zur erstmalig getrennten Untersuchung der Einflüsse von Ventilschließen und Verbrennungsdruck auf das Verschleißverhalten des Tribosystems VS /SR in Großgasmotoren. Nach Quellenlage sind für die Ventilphasen Schließen und Verbrennungsdruck die eigenschaftsbestimmenden Verschleißmechanismen sowie eine Zuordnung der Verschleißanteile am Gesamtventilverschleiß unzureichend bis nicht verstanden. Somit fehlt ein notwendiges Verständnis, um werkstoff- oder konstruktionstechnische Lösungsansätze für den Ventilverschleiß in hocheffizienten Großgasmotoren mit mechanischen Wirkungsgraden von bis zu 50 \% zu entwickeln. Darüber hinaus waren die Anteile der Komponentenverschleiße am Systemverschleiß von Interesse.

Zur Identifikation der eigenschaftsbestimmenden Verschleißmechanismen wurden mikrostrukturelle Untersuchungen an vier VSn mit unterschiedlicher Laufzeit aus demselben Großgasmotor an den Anfang der Arbeit gestellt. Zusammenfassung und Schlussfolgerungen finden sich im Kapitel 2.4. Aus den Erkenntnissen dieser Untersuchungen wurde ein neuer VVP abgeleitet. Entwicklung, Konstruktion und Aufbau des neuen Prüfstands wurden im Wesentlichen vorgestellt. Nach erfolgreicher Validierung hinsichtlich der Kriterien Ventilrotation, Kühlsystem, Verschleißerscheinungsformen und Verschleißrate wurden basierend auf einer kontrollierten Parametervariation erste Versuche bei $380^{\circ} \mathrm{C}$ an Luft durchgeführt. Die beiden Hauptparameter Ventilschließen und Verbrennungsdruck wurden 3stufig mit 0,2, 0,6 und 1,0 m/s bzw. 140, 180 und 220 bar variiert. Anschließend wurden die Verschleißproben mikrostrukturell charakterisiert und die Verschleißraten bestimmt, wobei die dominierenden Verschleißmechanismen für die beiden Hauptfaktoren Ventilschließen und Verbrennungsdruck im Hinblick auf die verwendeten Tribowerkstoffe identifiziert wurden. Darüber hinaus konnten aus den Versuchen neue Erkenntnisse in Bezug auf die Verschleißanteile beider Hauptfaktoren und Komponentenverschleiße unter Ventilschließen und Verbrennungsdruck gewonnen werden. Zentrale Aussage dieser Arbeit ist, dass das Ventilschließen als wichtigster Einflussfaktor auf den Ventilverschleiß für die tribotechnischen Werkstoffe Stellite ${ }^{\mathrm{TM}} 12$ und Pleuco $12 \mathrm{MV}$ betrachtet werden kann.

\subsection{Prüfstandsvalidierung}

Für die Aussagekraft von experimentellen Ergebnissen ist eine kontrollierte Parametervariation die Basis. Eine Prüfstandsvalidierug kann diese Kontrolle sichern, daher ist sie von besonderer Bedeutung. Neben den mechanischen Kriterien Ventilrotation und Kühlsystem bestimmen die tribologischen Kenngrößen Verschleißerscheinungsformen und Verschleißrate die Güte eines Verschleißprüfstands. Die Ventilrotation wurde mittels Kraftaufnehmer validiert. Im Falle einer Drehmomentmessung während des Ventilschließens würde dies eine Fehlfunktion des Prüfaufbaus bedeuten. Die Ergebnisse verdeutlichen ein vernachlässigbar kleines Drehmoment von $\pm 1 \mathrm{Nm}$. Als zweites mechanisches Kriterium wurde das Kühlsystem geprüft. Im Hinblick auf die späteren Versuchsreihen wurde eine 3-stufige 
Validierung der beiden Hauptparameter Ventilschließen und Verbrennungsdruck sowie der Temperatur durchgeführt. Bei 330,380 und $430{ }^{\circ} \mathrm{C}$ wurde die Schließgeschwindigkeit unter $0,2,0,6$ sowie $1,0 \mathrm{~m} / \mathrm{s}$ und der Verbrennungsdruck unter 140,180 sowie 220 bar geprüft. Die vorgegebenen Temperaturen konnten mit maximalen Deltas von $11 \mathrm{~K}$ für das Schließen und $5 \mathrm{~K}$ für den Verbrennungsdruck über alle Versuche in engen Grenzen realisiert werden. Daneben konnten für Schließen und Verbrennungsdruck mit maximalen Deltas von 321 bzw. $198 \mathrm{~K}$ über alle Versuche zwischen VS- und SR-Temperaturen die Literaturwerte eingehalten werden.

Als erste tribologische Kenngröße wurden die Verschleißerscheinungsformen validiert. Vergleichende Untersuchungen unter $0,6 \mathrm{~m} / \mathrm{s}$ bei RT und $330{ }^{\circ} \mathrm{C}$ demonstrieren mit unterschiedlichen Ergebnissen die Maschinenfähigkeit des VVPs, temperaturinduzierte Veränderungen zu simulieren. So beträgt die Verschleißmarkenbreite bei RT ca. $3 \mathrm{~mm}$ und bei $330^{\circ} \mathrm{C}$ ca. 6,6 mm. Ferner belegen Schuppen, Kuppen und kleine Löcher an der Oberfläche sowie ein sichtbarer Dehnungsgradient mit zerbrochenen Hartphasen bei $330^{\circ} \mathrm{C}$ eine andere Verschleißerscheinung im Vergleich zum Versuch bei RT mit fertigungstypischen Schleifriefen, gefältelten Schleifspitzen und vereinzelten Rissen in der karbidischen Dendritenstruktur. Der Temperatureinfluss wird gleichfalls für den Hauptparameter Verbrennungsdruck unter 180 bar bei RT und $330^{\circ} \mathrm{C}$ deutlich. Typische Schleifriefen mit geglätteten Schleifspitzen und einer nicht plastisch deformierten Randzone charakterisieren den Versuch bei RT. Hingegen bestimmen Schuppen, Kuppen und eine Triboschicht das Erscheinungsbild bei $330^{\circ} \mathrm{C}$. Die Verschleißmarkenbreite erreicht bei beiden Temperaturen die nominelle Sitzbreite. Neben der Nachbildung temperaturinduzierter Veränderungen demonstrieren die beobachteten Verschleißerscheinungen mit Kuppen und Schuppen in der Draufsicht sowie Dehnungsgradienten im Querschliff die gleichen Verschleißerscheinungen, wie sie an Teilen aus befeuerten Motorversuchen beobachtet werden konnten.

Als zweite tribologische Kenngröße wurde die Verschleißrate validiert. Repräsentativ wurden drei Versuche betrachtet. Die Ergebnisse der beiden Versuche unter $0,6 \mathrm{~m} / \mathrm{s}$ bei RT und $430^{\circ} \mathrm{C}$ mit Verschleißraten von 0,06 bzw. $1,60 \mathrm{~mm}^{3} / \mathrm{h}$ belegen den Temperatureinfluss auf das Verschleißverhalten des Tribosystems VS/SR. Einen signifikanten Einfluss des Faktors Schließgeschwindigkeit verdeutlicht der Versuch unter 1,2 m/s mit einer Verschleißrate von $3,33 \mathrm{~mm}^{3} / \mathrm{h}$ bei $450^{\circ} \mathrm{C}$. Im Vergleich mit den Verschleißraten aus den Motorversuchen (vgl. Kapitel 2.3.2) lassen sich Gemeinsamkeiten feststellen. So liegen die Verschleißraten dieser Komponenten mit Werten von 1,45 und 3,95 mm $3 / \mathrm{h}$ für die vergleichbare Position Einlass in der gleichen Größenordnung.

Als Fazit darf festgestellt werden, dass der neu entwickelte VVP die Verschleißerscheinungsformen und Verschleißraten, wie sie aus befeuerten Motorversuchen bekannt sind, qualitativ wie quantitativ nachbilden kann. 


\subsection{Prüfstandsversuche}

Erste Versuche am Beispiel von zwei 3-stufigen Versuchsreihen zum Einfluss von Ventilschließen und Verbrennungsdruck auf das Verschleißverhalten des Tribosystems VS/SR ergaben neue Erkenntnisse. Die Variation von Schließen und Druck erfolgte bei $380^{\circ} \mathrm{C}$ an Luft über $100 \mathrm{~h}$ mit 0,2, 0,6 und 1,0 m/s bzw. 140, 180 und 220 bar.

Verschleißerscheinungformen. Die Veränderungen in der tribologisch beanspruchten Randschicht stellen sich in Abhängigkeit von der Belastung unterschiedlich dar. Unter Ventilschließen kennzeichnen Schuppen, Kuppen und Löcher die Oberfläche des Grundkörpers VS, wobei aufgeschobene Schuppen an SID und SM sowie Mulden am SAD dominieren. Lokale Triboschichten sind an den Oberflächen zu beobachten. Der Gegenkörper SR weist eine vergleichsweise rau erscheinende Kontaktfläche auf, wobei Mulden und Ausbrüche dominieren. Unterhalb der Oberfläche von der VS lassen sich Dehnungsgradienten mit zerbrochenen metallkeramischen Hartphasen bis hin zu mechanisch gemischten Randschichten beobachten. Wohingegen der SR nur Risse in den Karbidstrukturen erkennen lässt. Unter Verbrennungsdruck präsentieren sich die Oberflächen von VS und SR erkennbar geglättet. Schuppen und Löcher bestimmen das Erscheinungsbild an VS und SR. Lokale Triboschichten bestehend aus $\mathrm{Co}, \mathrm{Cr}, \mathrm{Fe}, \mathrm{W}, \mathrm{C}$ und $\mathrm{O}$ wurden auf allen Oberflächen der VS nachgewiesen, wobei die Geschlossenheit über die Sitzbreite mit steigender Last zu zunehmen scheint. Die Mikrostruktur der VS wird widerum von zerbrochenen metallkeramischen Hartphasen in einem Dehnungsgradienten bestimmt. Risse in den Karbidstrukturen und Ausbrüche charakterisieren die Mikrostruktur des SRs.

Verschleißmechanismen. Für das Ventilschließen konnten die mechanisch-dominierten Verschleißmechanismen Adhäsion und Oberflächenzerrüttung mit dem Untermechanismus Delamination identifiziert werden. Diese beiden Mechanismen werden unter Verbrennungsdruck um den Mechanismus tribochemisch-dominierte Reaktion mit dem Untermechanismus Tribooxidation erweitert.

Quantitative Verschleißergebnisse. Die äquidistanten Tiefenprofile in Verbindung mit einer Zunahme der Profiltiefen über die Zeit bei unterschiedlichen Schließgeschwindigkeiten und Verbrennungsdrücken verdeutlichen ein lineares Verschleißverhalten. Daneben weisen beide Versuchsreihen keinen erhöhten Anfangsverschleiß auf, so dass ein Einlaufen des Systems, wie es für milden Verschleiß typisch ist, nicht beobachtet werden konnte. Gleichfalls kann anhand der Verrundung am SID des SR-Profils für beide Parameter ein Verlassen der Verschleißpartikel aus dem Reibspalt in Richtung SID vermutet werden. Hingegen lässt sich dieses Phänomen am SAD unter Verbrennungsdruck nicht erkennen. Somit kann die Verrundung am SAD des verschlissenen SR-Profils als typisches Merkmal des Ventilschießens angesehen werden. Daneben sind unter Ventilschleißen ein fehlender Verschleiß im Bereich des SIDs und eine zunehmende Konkavität im Tiefenprofil der VS über die Zeit mit steigender Last zu erkennen. Das Tiefenprofil des SRs verhält sich komplementär zur VS. 
Die ermittelten Verschleißraten aus beiden Versuchsreihen bestätigen einen signifikanten Einfluss von Schließgeschwindigkeit und Verbrennungsdruck auf den Ventilverschleiß. Für das Schließen wurde ein Anstieg der Komponentenverschleißraten von 0,2 auf 1,0 m/s in etwa um die Faktoren 4 und 10 für VS bzw. SR ermittelt. Dies kann auf die mechanisch-dominierten Verschleißmechanismen zurückgeführt werden. Als erste neue Erkenntnis für die untersuchten Tribowerkstoffe Stellite ${ }^{\mathrm{TM}} 12$ und Pleuco $12 \mathrm{MV}$ darf somit eine größere Zunahme des Komponentenverschleißes SR am Systemverschleiß festgehalten werden. Die zweite Erkenntnis folgt aus den Versuchen zum Verbrennungsdruck. Mit steigendem Druck wurde eine Abnahme der Verschleißrate von 140 auf 180 bar um ca. 30 \% für die VS gemessen. Hingegen blieb der SR-Verschleiß unverändert gleich. Der damit um ca. $20 \%$ reduzierte Systemverschleiß kann nahezu vollständig auf die VS zurückgeführt werden. Der Verschleißrückgang unter steigender Last bedeutet, dass die mechanisch-dominierten Mechanismen unter Ventilschließen um tribochemische Reaktionen unter Verbrennungsdruck erweitert wurden. Triboschichten unterbinden den metallischen Kontakt zwischen VS und SR. Aus dem Vergleich der Verschleißraten von Ventilschließen und Verbrennungsdruck resultieren zwei weitere neue Erkenntnisse. Betrachtet man die Systemverschleißraten für die anwendungstypischen Werte $0,6 \mathrm{~m} / \mathrm{s}$ und 180 bar mit 1,81 bzw. $2,61 \mathrm{~mm}^{3} / \mathrm{h}$, so scheinen die Faktoren Ventilschließen und Verbrennungsdruck gleichgewichtig. Damit können erstmals die Verschleißanteile von Ventilschließen und Verbrennungsdruck am Gesamtventilverschleiß zugeordnet werden, was die dritte neue Erkenntnis bedeutet. Die vierte und zugleich wichtigste Erkenntnis ist, dass der Systemverschleiß sensitiver auf die Schließgeschwindigkeit reagiert. So ändert sich der Systemverschleiß in den untersuchten Laststufen für Schließen und Verbrennungsdruck ca. um den Faktor 4,7 bzw. 1,3. Die betrachteten Laststufen entsprechen dem Stand der Technik für Großgasmotoren. Die zentrale Aussage dieser Arbeit ist somit, dass als wichtigster Einflussfaktor auf den Ventilverschleiß für die untersuchten tribotechnischen Werkstoffe Stellite $^{\mathrm{TM}} 12$ und Pleuco 12MW das Ventilschließen angesehen wird.

\subsection{Ausblick}

Die vorliegende Arbeit hat vordergründig die Entwicklung, Konstruktion und den Aufbau eines neuartigen VVPs unter dem Aspekt der Zusammenführung von Verschleißerscheinungsformen und Verschleißrate behandelt. Der Fokus wurde auf die getrennte Untersuchung der Ventilphasen Schließen und Verbrennungsdruck gelegt. Aus den ersten Versuchen konnten neue Erkenntnisse zur Ausrichtung zukünftiger Arbeiten gewonnen werden. Für die Entwicklung neuer Lösungsansätze zur Minimierung des Ventilverschleißes in hocheffizienten Großgasmotoren bedarf es weiterer Untersuchungen. Die Simulationen zum Ventilschließen und Verbrennungsdruck wurden bei konstanter Temperatur durchgeführt, hier sind Versuchsreihen mit variierender Temperatur zur Klärung des Temperatureinflusses erforderlich. Gleichsam sollten zukünftige Arbeiten den Einfluss verschiedener Atmosphären zum Gegenstand haben, um den Atmosphäreneinfluss typischer Bestandteile der Ansaug- und Abgasluft wie Kohlendioxid, Stickstoff, Wasserdampf oder Ölnebel auf tribochemische 
Prozesse besser zu verstehen. Damit kann die Bildung möglicher Triboschichten, wie sie von [12] und [60] beobachtet wurden, besser beurteilt werden. Aus Versuchen an Argon lassen sich möglicherweise neue Erkenntnisse zur Tribooxidation und Eindringen von Sauerstoff in die tribotechnischen Werkstoffe gewinnen.

Für weitere Untersuchungen erscheint es ebenso erforderlich den Einfluss des Verbrennungsdrucks auf das Verschleißverhalten durch eine Parameterstudie besser zu verstehen. Im Ergebnis sollten z.B. Fragen beantwortet werden können wie: Welche Größe des Fehlerindikators der Verschleißrate systemimmanent ist? Welcher globale Trend stellt sich bei höheren Drücken bis zu 300 bar ein?

Mit dem Abrastern mehrstufiger Parametervariationen bietet sich die Möglichkeit zur Erstellung eines Parameterkennfelds. Somit kann eine Abschätzung des Verschleißverhalten für untersuchte tribotechnische Werkstoffe ermöglicht werden.

Lösungsansätze beruhend auf der Bildung kompaktierter Triboschichten aus Verbrennungsrückständen werden zukünftig wahrscheinlich nicht ausreichen, da Verbrennungsprozesse tendenziell ruß- und ascheärmer werden, um verschärfte Emissionsvorschriften hinsichtlich $\mathrm{CO}_{2}, \mathrm{NO}_{x}, \mathrm{SO}_{x}$ und Feinstaub applikationsübergreifend zu erfüllen. Daher sollte die Untersuchung alternativer Werkstoffkombinationen zur Reduktion der dissipierten Reibleistung ein zukünftiges Ziel sein. Ebenso bietet die Kontaktsituation zwischen VS und SR weitergehendes Optimierungspotenzial.

Nach dem Lesen dieser Arbeit wird sicher verständlich, dass der Ventilverschleiß aufgrund seiner multifaktoriellen Einflüsse keineswegs einfach zu lösen ist. Vermutlich wird es aufgrund der gegensätzlichen Werkstoffanforderungen für Gleit- und Stoßverschleiß keine Patentlösung geben. Abschließend darf zukünftigen Arbeiten zu diesem Thema der gezielte Komponentenversuch empfohlen werden. 


\section{$7 \quad$ Literaturverzeichnis}

[1] R. Ollus, H. Tienhaara und K. Portin, „Technologies for flexible and efficient engines, “ 12th International MTZ Conference on Heavy-Duty Engines, pp. 1-22, 27-28 November 2017.

[2] N. Böckhoff, D. Mondrzyk und S. Terbeck, „Kontinuierliche Weiterentwicklung des 51/60G zum 51/60G TS der MAN Diesel \& Turbo SE," Proceedings 10th Dessau Gas Engine Conference, pp. 61-71, 6-7 April 2017.

[3] R. Lejon, „W31 engine - properties, some technical details and first experiences, “ 40. ISF-Tagung Neue Entwicklungen und Betriebserfahrungen in der Schiffsbetriebstechnik, pp. 84-99, 7-8 June 2018.

[4] A. Ludu, „Dual fuel engine technoloy trends, “ 6th AVL Large Engine TechDays, pp. 128, 6-7 Mai 2014.

[5] S. Goto, „Advanced development of Niigata medium-speed dual-fuel and gas engine with 1 to $6 \mathrm{MW}$ range suitable for marine propulsion and power generation," 6th AVL Large Engine Techdays, 6-7 May 2014.

[6] J. Burke, „https://dieselgasturbine.com,“ KHL Group LLP, 11 Mai 2016. [Online]. Available: https://dieselgasturbine.com/powerful-first-texas/. [Zugriff am 31 Mai 2018].

[7] Diesel \& Gas Turbine Worldwide, „https://dieselgasturbine.com, “ KHL Group LLP, 20 März 2018. [Online]. Available: https://dieselgasturbine.com/wartsila-hybridpowerplant/. [Zugriff am 31 Mai 2018].

[8] M. Wideskog, „Wärtsilä hybrid solutions, “ 8th AVL Large Engine Techdays, pp. 1-12, 67 April 2018.

[9] European Engine Power Plants Association, „A research roadmap for engine power plants, Draft versions 2 - 14.06.2016,“ 2016.

[10] A. Lippert, S. Laiminger und M. Schneider, "Großgasmotoren im Spannunsfeld der erneuerbaren Energie und Elektromobilität, stirbt der Verbrennungsmotor aus?,“ 16. Tagung "Der Arbeitsprozess des Verbrennungssmotors", pp. 572-583, 28-29 September 2017.

[11] A. Rajewski und G. Niewinski, „Reciprocating engines as a source of flexibility in a power system," Rynek Energii, Nr. 5, pp. 80-86, 2017.

[12] P. Forsberg, F. Gustavsson, P. Hollman und S. Jacobson, „Comparison and analysis of protective tribofilms found on heavy duty exhaust valves from field and made in a test rig," Wear, Bd. 302, Nr. 1-2, pp. 1351-1359, 2013.

[13] K.-H. Habig, Verschleiß und Härte von Werkstoffen, Carl Hanser Verlag, München Wien, 1980. 
[14] H. Czichos und K.-H. Habig., Hrsg., Tribologie-Handbuch Tribometrie, Tribomaterialien, Tribotechnik, 4. Hrsg., Springer Vieweg, 2015.

[15] L. Deters, A. Fischer, E. Santner und U. Stolz, GfT-Arbeitsblatt 7: Tribologie. Verschleiß, Reibung. Definitionen, Begriffe, Prüfug, Gesellschaft für Tribologie, 2002.

[16] K. Sommer, R. Heinz und J. Schöfer, Verschleiß metallischer Werkstoffe, 2. Hrsg., Springer Vieweg, 2014.

[17] G. Stachowiak, Wear - Materials, Mechanisms and Practice, 1. Hrsg., John Wiley \& Sons, Ltd., 2005.

[18] E. Rabinowicz, Friction and Wear of Materials, 2. Hrsg., John Wiley and Sons, Inc., 1995.

[19] H. P. Jost, „Lubrication (Tribology) - A Report of the Present Position and Industry`s Needs," London, 1966.

[20] W. Bartz, Zur Geschichte der Tribologie, Bd. 1, Expert-Verlag, 1988.

[21] D. Dowson, History of Tribology, 2. Hrsg., London and Bury St Edmunds: Professional Engineering Publishing Ltd., 1998.

[22] K. H. Zum Gahr, Microstructure and Wear of Maetrials, Bd. Tribology Series 10, Elsevier Science Publishers B.V., 1987.

[23] BMFT-Report, „Tribologie, Reibung-Verschleiß-Schmierung,“ Bonn, 1976.

[24] ASME-Report, „Strategy for Energy Conservation through Tribology, “ ASME Publications Department, New York, 1977.

[25] BMFT-Report, „Damit Rost und Verschleiß nicht Milliarden fressen, “ Bonn, 1984.

[26] H. P. Jost und J. Schofield, „Energy saving through tribology: A techno-economic study," Proceeding of the Institution of Mechanical Engineers, Bd. 195, Nr. 1, pp. 151$173,1981$.

[27] N. Welsh, „The dry wear of steels II. Interpretation and special features," Philosophical Transactions of the Royal Society A, Bd. 257, Nr. 1077, pp. 51-70, 7 Januar 1965.

[28] R. Bayer, Mechanical Wear Fundamentals and Testing, 2. Hrsg., I. N. Y. B. Marcel Dekker, Hrsg., 2004.

[29] P. J. Blau, Friction science and technology: From concepts to applications, 2. Hrsg., CRC Press Taylor \& Francis Group, LLC, 2009.

[30] R. Stribeck, „Die wesentlichen Eigenschaften der Gleit- und Rollenlager, “ Zeitschrift Verein Deutscher Ingenieure, Nr. Vol. 46, pp. 1341-1348, 1902.

[31] O. Pigors, Werkstoffe in der Tribotechnik: Reibung, Schmierung und Verschleißbeständigkeit von Werksoffen und Bauteilen, Leipzig: Deutscher Verlag für Grundstoffindustrie, 1993.

[32] G. Schmaltz, Technische Oberflächenkunde, Berlin: Springer-Verlag, 1936. 
[33] F. Bowden und D. Tabor, Reibung und Schmierung fester Körper, Springer-Verlag, Berlin, Göttigen, Heidelberg, 1959, p. 1.

[34] F. P. Bowden und D. Tabor, "The area of contact between stationary and moving surfaces," Proceeding of the Royal Society of London. Seriea A, Mathematical and Physical Sciences, Bd. 169, Nr. 938, pp. 391-413, 1939.

[35] F. Bowden und D. Tabor, „Friction, Lubrication and wear: A Survey of work during the last decade," British Journal of Applied Science, Bd. 17, Nr. 12, pp. 1521-1544, 1966.

[36] R. Holm, Electric contacts handbook, 3. Hrsg., Springer Verlag, 1958.

[37] J. A. Greenwood und J. B. P. Williamson, „Contact of Nominally Flat Surfaces, “ Proceedings of the Royal Society London A, Nr. 295, pp. 300-319, 6 December 1966.

[38] D. J. Whitehouse und J. F. Archard, "The properties of random surfaces of significance in their contact," Proceeding of the Royal Society London, Bd. 316, Nr. 1524, pp. 97121, 1970.

[39] D. Tabor, The hardness of Metals, Oxford University Press, 1951.

[40] D. Tabor, „Hardness of solids, “ Review of Physics in Technology, Bd. 1, Nr. 3, pp. 145179, 1970.

[41] T. F. J. Quinn, "The effect of "hot-spot" temperatures on the unlubricated wear of steel," ASLE Transactions, Nr. 10, pp. 158-168, 1967.

[42] J. Burwell, „Survey of possible wear mechanisms, “ Wear, Bd. 1, Nr. 2, pp. 119-141, October 1957.

[43] R. Lewis und R. S. Dwyer-Joyce, Automotive Engine Valve Recession, London: Professional Engineering Publishing Ltd., 2002.

[44] N. P. Suh, „An overview of the delamination theory of wear, “ Wear, Bd. 44, Nr. 1, pp. 1-16, 1977.

[45] M. Sikorski, "Correlation of the coefficient of adhesion with various physical and mechanical properties of metals, "Journal of Fluids Engineering, Nr. D85, pp. 279-285, 1963.

[46] D. Buckley, Surface effects in adhesion, friction, wear and lubrication, Amsterdam: Elsevier Scientific Publishing Company, 1981.

[47] J. Archard und W. Hirst, „The Wear of Metals under Unlubricated Conditions," Proceedings of the Royal Society, Nr. 236, pp. 397-410, 1956.

[48] M. Fink, "Chemische Aktivierung, nicht durch Temperatur, sondern durch plastische Verformung, als Ursache reibchemischer Reaktionen bei Metallen," FortschrittBerichte VDI-Zeitschrift, Bd. Nr. 3, Nr. Reihe 5, 1967.

[49] M. Fink und H. Krause, "Mechanisch-chemische Reaktionen bei der Abnutzung von St 60, V2A und Manganhartstahl," Fortschritt-Berichte VDI-Zeitschrift, Bd. Reihe 5, 1967.

[50] J. Heidemeyer, „Einfluß der plastischen Verformung von Metallen bei Mischreibung auf die Geschwindigkeit ihrer chemischen Reaktionen," Schmiertechnik Tribologie, Nr. 22, pp. 84-90. 
[51] F. H. Stott und G. C. Wood, "The influence of oxides on the friction and wear of alloys," Tribology International, Bd. 11, Nr. 4, pp. 211-218, 1978.

[52] R. Lewis, R. Dwyer-Joyce und G. Josey, „Design and Development of a Bench Test-Rig for Investigating Diesel Engine Inlet Valve and Seat Insert Wear," AUSTRIB '98, Tribology at Work: Proceedings of the 5th International Tribology Conference, pp. 365370, 1998.

[53] D. A. Rigney, "Sliding wear of metals, " Annual Review of Material Science, Bd. 18, pp. 141-163, 1988.

[54] P. J. Blau, Friction and wear transitions of materials: break-in, run-in, wear-in, Noyes Publications, 1989.

[55] T. S. Eyre und D. Maynard, "Surface aspects of unlubricated metal-to-metal wear, “ Wear, Nr. 18, pp. 301-310, 1971.

[56] B. Chattopadhyay und G. C. Wood, "The transient oxidation of alloys, " Oxidation of Metals, Bd. 2, Nr. 4, pp. 373-399, Dezember 1970.

[57] F. H. Stott, "The role of oxidation in the wear of alloys," Tribology International, Bd. 31, Nr. 1-3, pp. 61-71, Januar 1998.

[58] F. Stott, „High-temperature sliding wear of metals, “ Tribology International, Bd. 35, Nr. 8, pp. 489-495, August 2002.

[59] J. F. Archard, „The temperature of rubbing surfaces,“ Wear, Bd. 2, Nr. 6, pp. 438-455, Oktober 1959.

[60] A. Renz, D. Kürten und O. Lehmann, „Wear of hardfaced valve spindles in highly loaded stationary lean-burn large bore gas engines, "Wear, Bde. \%1 von \%2376-377, pp. 1652-1661, 2017.

[61] M. Godet, „The third-body approach: A mechnanical view of wear, “ Wear, Bd. 100, pp. 437-452, 1984.

[62] J. Jiang, F. H. Stott und M. Stack, „The role of triboparticulates in dry sliding wear, “ Tribology International, Bd. 31, Nr. 5, pp. 245-256, Mai 1998.

[63] A. Iwabuchi, „The role of oxide particles in the fretting wear of mild steel,“ Wear, Bd. 151, Nr. 2, pp. 301-311, Dezember 1991.

[64] A. Iwabuchi, H. Kubosawa und K. Hori, „The dependence of the transition from severe to mild wear on load and surface roughness when oxide particles are supplied before sliding,"Wear, Bd. 139, Nr. 2, pp. 319-333, August 1990.

[65] K. Hiratsuka und K. Muramoto, „Role of wear particles in severe-mild wear transition," Wear, Bd. 259, Nr. 1-6, pp. 467-476, 2005.

[66] J. Glascott, F. H. Stott und G. C. Wood, "The effectiveness of oxides in reducing sliding wear of alloys," Oxidation Metals, Bd. 24, Nr. 3-4, pp. 99-114, 1985.

[67] D. A. Rigney, „Transfer, mixing and associated chemical and mechanical processes during sliding of ductile materials," Wear, Bd. 245, Nr. 1-2, pp. 1-9, 2000. 
[68] F. H. Stott, D. S. Lin und G. C. Wood, „"Glazes" produced on nickel-base alloys during high temperature wear," Nature Physical Science, Bd. 242, Nr. 118, pp. 75-77, 1973.

[69] I. A. Inman, S. Datta, H. L. Du, J. S. Burnell-Gray und Q. Luo, „Microscopy of glazed layers formed during high temperature sliding wear at $750{ }^{\circ} \mathrm{C}, “$ Wear, Bd. 254, Nr. 5-6, pp. 461-467, 2003.

[70] F. H. Stott, D. S. Lin und G. Wood, „The structure and mechanism of formation of the "glaze" oxide layers produced on nickel-based alloy during wear at high temperatures," Corrosion Science, Bd. 13, Nr. 6, pp. 449-454, 1973.

[71] F. H. Stott und M. P. Jordan, "The effects of load and structure hardness on the development and maintenance of wear-protetctive layers during sliding at elevated temperatures," Wear, Bd. 250, Nr. 1-12, pp. 391-400, 2001.

[72] P. Heilmann, W. A. T. Clark und D. A. Rigney, "Orientation determination of subsurface cells generated by sliding," Acta Metallurgica, Bd. 31, Nr. 8, pp. 12931305, 1983.

[73] P. J. Blau, „Four great challenges confronting our understanding and modeling of sliding friction," Tribology Series, Bd. 34, pp. 117-128, 1998.

[74] I. M. Allam, „Solid lubricants for applications at elevated temperatures, “ Journal of Materials Sciene, Bd. 26, Nr. 15, pp. 3977-3984, 1991.

[75] R. Bürgel, H. J. Maier und T. Niendorf, Handbuch Hochtemperatur-Werkstofftechnik, 5. Hrsg., Springer Verlag, 2015.

[76] I. Simon, H. O. McMahon und R. J. Bowen, „Dry Metallic Friction as a Function of Temperature Between $4.2 \mathrm{~K}$ and $600 \mathrm{~K}$," Journal of Applied Physics, Bd. 22, pp. 177184, 1951.

[77] A. Fischer, "Sliding Wear of Metallic Materials at 25 and $550^{\circ} \mathrm{C}$, "in MATERIALS RESEARCH SOCIETY SYMPOSIUM PROCEEDINGS, 1988.

[78] M. G. Peterson, J. J. Florek und R. E. Lee, „Sliding Characteristics of Metals at High Temperaturs," A S L E Transactions, Bd. 3, Nr. 1, pp. 101-115, 1960.

[79] I. A. Inman, P. K. Datta, H. L. Du, J. S. Burnell-Gray, S. Pierzgalski und Q. Luo, „Studies of high temperature sliding wear of metallic dissimilar interfaces, "Tribology International, Bd. 38, Nr. 9, pp. 812-823, 2005.

[80] A. Fischer, „Mechanisms of high temperature sliding abrasion of metallic materials, “ Wear, Bd. 152, Nr. 1, pp. 151-159, 1992.

[81] P. J. Blau, „Elevated-temperature tribology of metallic materials, “ Tribology International, Bd. 43, Nr. 7, pp. 1203-1208, 2010.

[82] B. Yucel, „High temperature sliding wear behaviour of Inconel 617 and Stellite 6 alloys," Wear, Bd. 269, Nr. 9-10, pp. 664-671, 2010.

[83] P. J. Blau und T. M. Brummett, „High-temperature oxide regrowth on mechanically damaged surfaces," Tribology Letters, Bd. 32, Nr. 3, pp. 153-157, 2008. 
[84] I. A. Inman, S. R. Rose und P. K. Datta, „Studies of high temperature sliding wear of metallic dissimilar interfaces II: Incoloy MA956 versus Stellite 6," Tribology International, Bd. 39, Nr. 11, pp. 1361-1375, 2006.

[85] C. Rynio, H. Hattendorf, J. Klöwer und G. Eggeler, „The evolution of tribolayers during high temperature sliding wear," Wear, Bd. 315, Nr. 1-2, pp. 1-10, 2014.

[86] F. H. Stott, J. Glascott und G. C. Wood, „Factors affecting the progressive development of wear-protective oxides on iron-base alloys during sliding at elevated temperatures," Wear, Bd. 97, Nr. 1, pp. 93-106, 1984.

[87] J. Jiang, F. H. Stott und M. M. Stack, „A mathematical modell for sliding wear of metals at elevated temperatures, "Wear, Bde. \%1 von \%2181-183, pp. 20-31, 1995.

[88] I. A. Inman, P. S. Datta, H. L. Du, K. C. Kübel, P. D. Wood und F. T. Mahi, „1.15 - High Temperature Tribocorrosion," in Shreir's Corrosion, Elsevier, 2017, pp. 331-398.

[89] H. C. Meng und K. C. Ludema, „Wear models and predictive equations: their form and content, “ Wear, Bde. \%1 von \%2181-183, pp. 443-457, 1995.

[90] K. Holmberg und A. Laukkanen, Handbook of Lubrication and Tribology, 2 Hrsg., Bd. Volume II, 2012.

[91] P. A. Engel, Impact Wear of Materials, 2. Hrsg., Elsevier Scientific Publishing Company, Amsterdam Oxford New York, 1978.

[92] P. A. Engel, „Percussive impact wear: A study of repetitively impacting solid components in engineering," Tribology International, pp. 169-176, 1978.

[93] P. A. Engel, T. H. Lyons und J. L. Sirico, „Impact wear model for steel specimens, “ Wear, pp. 185-201, 1973.

[94] C. J. Studman und J. E. Field, „A repeated impact testing machine, “ Wear, pp. 373381, 1977.

[95] S. L. Rice, „Reciprocating impact wear testing apparatus,“ Wear, pp. 85-95, 1977.

[96] E. Rabinowicz, „Metals transfer during statuc loading and impacting," Proceeding Physics Society (London), 65B, pp. 630-640, 1952.

[97] A. W. J. De Gee, C. P. L. Commissaris und J. H. Zaat, ",The wear of sintered aluminium powder (SAP) under conditions of vibrational contact,“ Wear, pp. 535-550, 1964.

[98] R. S. Montgomery, „The mechanism of percussive wear of tungsten carbide composites," Wear, pp. 309-329, 1968.

[99] R. G. Bayer, P. A. Engel und J. L. Sirico, „Impact wear testing machine,“ Wear, pp. 343354, 1972.

[100] R. Blickensderfer und J. H. Tylczak, „A large-scale impact spalling test,“ Wear, pp. 361373, 1983.

[101] G. M. Sorokin und I. I. Matyushima, „Wear tests carried out by impact of an abrasive on a massive sample,"Zavod. Lab., Bd. 37, pp. 218-220, 1971.

[102] G. M. Sorokin, „Wear resistance criteria for steel under impact on abrasive," Machinoved., Bd. 3, pp. 111-115, 1973. 
[103] M. M. Khruschov, „Principles of abrasive wear,“ Wear, Bd. 28, pp. 69-88, 1974.

[104] K. Wellinger und H. Breckel, „Kenngrössen und Verschleiss beim Stoss metallischer Werkstoffe," Wear, Bd. 13, pp. 257-281, 1969.

[105] M. Scherge, D. Shakhvorostov und K. PöhImann, „Fundamental wear mechanism of metals,"Wear, Nr. 255, pp. 395-400, 2003.

[106] M. Scherge und K. Pöhlmann, „Verschleißmechanismen bei moderater und extremer Grenzreibung," Materialwissenschaften und Werkstofftechnik, Bd. 35, Nr. 10-11, pp. 610-613, 2004.

[107] M. Belin, J. L. Mansot und J. M. Martin, „Correlation between wear rate and structural data of debris," in Interface Dynamics, Bd. 12. Tribology Series, Elsevier, Amsterdam, 1987.

[108] Y. Berthier, „Third-body-reality - consequences and use of the third-body concept to solve friction and wear problems," in Wear - Materials, Mechanisms and Practice, John Wiley \& Sons. Ltd, 2005.

[109] D. Shakhvorostov, K. Pöhlmann und M. Scherge, „Structure and mechanical properties of tribologically induced nanolayers," Wear, Bd. 260, pp. 433-437, 2006.

[110] H. Grohe, Otto- und Dieselmotoren, 12. Hrsg., Vogel Buchverlag, 2000.

[111] O. Riemenschneider, „Peak performance in a broad operational envelope, " 8 th AVL Lagre Engine Techdays, 6-7 April 2018.

[112] A. Urlaub, Verbrennungsmotoren, 2. Hrsg., Springer Berlin Heidelberg, 1995.

[113] R. van Basshuysen und F. Schäfer, Handbuch Verbrennungsmotor, Springer Fachmedien Wiesbaden, 2015.

[114] H. Tschöke,, K. Mollenhauer und R. Maier, Handbuch Dieselmotoren, Springer Fachmedien Wiesbaden, 2018.

[115] H. Eichlseder, M. Klüting und W. Piock, Grundlagen und Technologien des Ottomotors, SpringerWienNewYork, 2008.

[116] K. Kuiken, Diesel Engines for ship propulsion and power plants from 0 to $100.000 \mathrm{~kW}$, Bd. I, Target Global Energy Training, 2008.

[117] F. Zacharias, Gasmotoren, Würzburg: Vogel-Verlag, 2001.

[118] Fachbereich WD 8: Umwelt, Naturchutz, Reaktorsicherheit, Bildung und Forschung, „CO2-Bilanzen verschiedener Energieträger im Vergleich," Wissenschaftliche Dienste des Deutschen Bundestages, 2007.

[119] C. Poensgen, „Development of the MAN Diesel \& Turbo engine portfolio and future challenges, "12th International MTZ Conference o Heavy-Duty Engines, 27-28 November 2017.

[120] W. Müller und T. Kammerdiener, „Aktuelle Trends in der Großmotorenentwicklung,“ 16th Conference "The Working Process of the Internal Combustion Engine", pp. 50-62, 28-29 September 2017. 
[121] R. Pischinger, M. Klell und T. Sams, Thermodynamik der Verbrennungskraftmaschine, 3, illustriert Hrsg., Springer-Verlag, 2009.

[122] Mahle GmbH, Ventiltrieb: Systeme und Komponenten, Mahle GmbH, Hrsg., Springer Verlag, 2012.

[123] X. Dong, „Zur Berechnung des geometrischen Öffnungsquerschnitts von Kegelventilen," Motortechnische Zeitschrift MTZ, Nr. 46, pp. 221-224, 1985.

[124] G. Herdin, Gasmotoren., Technische Universität Graz, Graz: Vorlesungsskriptum, 2003.

[125] R. Müller und R. Weintz, „Ventilwerkstoffe für Verbrennungsmotoren,“ Materialwissenschaft ud Werkstofftechnik, Bd. 29, Nr. 3, pp. 97-130, 1998.

[126] G. N. Beddoes, „Valve materials and design, " Surface Engineering, Bd. 9, Nr. 1, pp. 4450, 1993.

[127] M. Atapour und F. Ashrafizadeh, „Tribology and cyclic oxidation behavior of plasma nitrided valve steel," Surface Coating Technology, Bd. 202, pp. 4922-4929, 2008.

[128] E. Lugscheider und D. Hofmann, „Hartlegierungen - Untersuchung von WIG- und Pulver-Plasma-auftraggeschweißten Hartlegierungen für Ventilsitzbeschichtungen, “ Forschungsvereinigung Verbrennungskraftmaschinen e.V., 1989.

[129] Y. Zhu, Z. Yin und H. Teng, „Plasma cladding of stellite 6 powder on Ni76Cr19AITi exhausting valve," Transactions of Nonferrous Metals Society of China, Bd. 17, pp. 3540, 2007.

[130] J. Chen, X. Y. Li, T. Bell und H. Dong, „Improving the wear properties of Stellite 21 alloy by plasma surface alloying with carbon and nitrogen," Wear, Bd. 264, Nr. 3-4, pp. 157165, 2008.

[131] Y. Wang, Introduction to engine valvetrains, S. International, Hrsg., Warrendale, PA, 2007.

[132] S. K. Schaefer, J. M. Larson, L. F. Jenkins und Y. Wang, „Evolution of heavy duty engine valves - materials and design," Valvetrain System Design and Materials, International Symposium, pp. 129-139, 1997.

[133] J. G. Sun, J. M. Zhang, M. J. Andrews, J. S. Trethewey, N. S. L. Phillips und J. A. Jensen, „Evaluation of Silicon-Nitride ceramic valves," International Journal of Applied Ceramic Technology, pp. 164-180, 2008.

[134] L. Aitchison, „Valve failures and valve steels in internal-combustion engines, “ Automobile Engineer, Nr. 9 / n 132, pp. 401-410, 1919.

[135] S. D. Heron und B. L. Beall, „Distortion reduced by use of flexible valve seats,“ Machine Design, Bd. 9, Nr. n 4, pp. 31-33, 1937.

[136] J. Newton und M. Tauschek, „Valve seat distortion,“ SAE Technical Paper 530049, 1953.

[137] R. W. Fricke und C. Allen, „Repetitive impact wear of steels, “ Wear, Bd. 162-164, pp. 837-847, 1993. 
[138] M. Malatesta, G. Barber, J. Larson und S. Narasimhan, „Development of a laboratory bench test to simulate seat wear of engine poppet valves," Tribology Transactions, Bd. 36, Nr. 4, pp. 627-632, 1993.

[139] L. Mascarenhas, J. Gomes, V. Beal, A. Portela, C. Ferreira und C. Barbosa, „Design and operation of a high temperature wear test apparatus for automotive valve materials," Wear, Bde. \%1 von \%2342-343, pp. 129-137, 2015.

[140] J. Guo, W. Zhang und D. Zou, „Investigation of dynamic characteristics of a valve train system," Mechanism and machine theory, Bd. 46, pp. 1950-1969, 2011.

[141] T. Ootani, N. Yahata, A. Fujiki und A. Ehira, „Impact wear characteristics of engine valve and valve seat insert materials at high temperature (impact wear tests of austenitic heat-resistant steel SUH36 against Fe-base sintered alloy using plane specimens),“ Wear, Bd. 188, pp. 175-184, 1995.

[142] K. Chun, J. Kim und J. Hong, "A study of exhaust valve and seat insert wear depending on cycle numbers," Wear, Bd. 263, Nr. 7-12, pp. 1147-1157, 2007.

[143] T. Slatter, H. Taylor, R. Lewis und P. King, „,The influence of laser hardening on wear in the valve and valve seat contact," Wear, Bd. 267, pp. 797-806, 2009.

[144] K. Zinner, „Investigations concerning wear of inlet valve seats in diesel engines,“ ASME Paper, Nr. 63-OGP-1, 1963.

[145] J. Pope, „Techniques used in achieving a high specific airflow for high-output mediumspeed diesel engines, "Journal of Engineering for Power, Bd. 89, Nr. 2, pp. 265-275, 1967.

[146] R. J. Mathis, R. W. Burrham, S. Ariga und R. D. Brown, „Gas-engine durability improvement. Final report, October 1985-September 1989. Volume 1,“ United States: N. p., 1990.

[147] Y. Wang, S. Narasimhan, J. Larson, J. Larson und G. Barber, "The effect of the operating conditions on heavy duty engine valve seat wear," Bd. 201, Nr. 1-2, pp. 1525, 1996.

[148] P. Forsberg, D. Debord und S. Jacobson, „Quantification of combustion valve sealing interface sliding - A novel experimental technique and simulations, “ Wear, Bd. 69, pp. 150-155, 2014.

[149] E. F. De Wilde, „Investigation of engine exhaust valve wear,“ Wear, Bd. 10, pp. 231244, 1967.

[150] R. Zhao, G. C. Barber, Y. S. Wang und J. E. Larson, „Wear mechanism analysis of engine exhaust valve seats with a laboratory simulator," Tribology Transactions, Bd. 40, pp. 209-218, 1997.

[151] Y. S. Wang, J. M. Narasimhan, J. M. Larson und S. K. Schaefer, „Wear and wear mechanism simulation of heavy-duty engine intake valve and seat inserts, "Journal of Materials Engineering and Performance, Bd. 7, Nr. 1, pp. 53-65, 1998. 
[152] X. Liang, G. Strong, D. Eickmeyer und K. Myers, „A study of valve seat insert wear mechanisms," SAE Technical Paper Series, 1999.

[153] S. R. J. Saunders, M. Monteiro und F. Rizzo, "The oxidation behaviour of metals and alloys at high temperatures in atmospheres containing water vapour: A review, “ Progress in Materials Science, Bd. 53, pp. 775-837, 2008.

[154] M. I. Karamangil, A. Avci und H. Bilal, „Investigation of the effect of different carbon film thickness on the exhaust valve," Heat Mass Transfer, Bd. 44, pp. 587-598, 2008.

[155] C. A. Mantey, A. Mezzarano und A. Kolkemo, „Exhaust valve \& valve seat insert: Development for an industrial LGP application," SAE Technical Paper Series, 2009.

[156] P. Forsberg, P. Hollman und S. Jacobson, „Wear mechanism study of exhaust valve system in modern heavy duty combustion engines, "Wear, Bd. 271, pp. 2477-2484, 2011.

[157] O. Lehmann und A. Renz, „Valve wear in lean-burn large bore gas engines - From engine tests of components to a unique tribological test rig, " CIMAC Congress, p. 2016 | 231, 6-10 June 2016.

[158] K. Chun, Y. Kim, J. Hong, J. Kim, S. Hyung und C. Shin, „A Study of Wear in Engine Exhaust Valve Depending on Valve Materials Using a Laboratory Simulator, "SAE Technical Paper 2007-01-0944, 2007.

[159] R. Lewis und R. S. Dwyer-Joyce, „Wear of Diesel Engine Inlet Valves and Seat Inserts," Proceedings of the Institution of Mechanical Engineers, Part D: Journal of Automobile Engineering, Bd. 216, Nr. 205, pp. 205-216, 2002.

[160] O. Lehmann, M. Scherge und A. Renz, „Wear mechanism study of Stellite-hardfaced combustion inlet valve spindles in lean-burn large bore gas engines," 9th Dessau Gas Engine Conference, pp. 137-145, 16-17 April 2015.

[161] F. Sass, Geschichte des Deutschen Verbrennungsmotorenbaues, Springer-Verlag Berlin Heidelberg GmbH, 1962.

[162] A. Ramalho, P. Kapsa, G. Bouvard, J.-C. Abry, T. Yoshida, M. Charpentier und M. Bourgeois, „Effect of temperatures up to $400{ }^{\circ} \mathrm{C}$ on the impact-sliding of valve-seat contacs," Wear, Bd. 267, pp. 777-780, 2009.

[163] V. A. Johnson und C. W. Galen, „Diesel Exhaust Valves,“ SAE Technical Papers, Nr. 660034, pp. 171-179, 1966.

[164] S. L. Narasimhan und J. M. Larson, „Valve Gear Wear and Materials, " SAE Technical Papers, Bd. 851497, 1985.

[165] E. B. Arnold, M. A. Bara, D. M. Zang, T. N. Tunnecliffe und J. Oltean, „Development and Application of a Cycle for Evaluating Factors Contributing to Diesel Engine Valve Guttering," SAE Technical Papers, Nr. 880669, 1988.

[166] C. G. Williams, „Factors Influencing Wear of Valve Seats in Internal-Combustion Engines," Engineering, Bd. 143, Nr. 3719, pp. 475-476, 1937. 
[167] M. S. Satish, T. E. McGreevy, J. J. Buettner, D. A. Cusac, G. J. Kaufmann, R. P. Maloney, B. A. Cull, J. R. Davenport, B. Hamdan, D. E. Hackett, R. A. Peterson, G. L. Spadin, L. L. Randle und M. L. Lusher, „Apparatus and Method for Testing Internal Combustion Engine Valves“. USA Patent US 6,647,770 B2, 2003.

[168] P. Forsberg, P. Hollman und S. Jacobson, „Wear Study of Coated Heavy Duty Exhaust Valve Systems in a Experimental Test Rig," SAE Technical Paper 2012-01-0546, 2012.

[169] P. Forsberg, „Combustion Valve Wear - A Tribological Study of Combustion Valve Sealing Interfaces, " Digital Comprehensive Summaries of Uppsala Dissertations from the Faculty of Science and Technology, 2013.

[170] U. Astrand, H. Aatola und J.-M. Myllykoski, „,Wärtsilä 31 - world`s most efficient fourstroke engine," CIMAC Congress, p. 2016 | 225, 6-10 June 2016.

[171] A. Gurr, A. Atkins, D. Rawlins und R. Morgan, „The 60\% efficiency reciprocating engine: A modular alternative to large scale combined cycle power, " CIMAC Congress, p. 2016 | 267, 6-10 June 2016.

[172] H. Sorger, "The modular large engine for future markets," 8th AVL Large Engines Techdays, 11-12 April 2018.

[173] T. Motoda, „Fusion of hybrid and LNG, " 8th AVL Large Engine Techdays, 10-11 April 2018.

[174] G. Würsig, „40. ISF-Tagung - Neue Entwicklungen und Betriebserfahrungen in der Schiffsbetriebstechnik, " in Alternative Brennstroffe in der Schifffahrt - Welche Trends gibt es wirklich in den nächsten Jahren, Flensburg, Germany, 2018.

[175] T. Slatter, R. Lewis und A. H. Jones, „The influence of induction hardening on the impact wear resistance of compacted graphite iron (CGI), “ Wear, Bd. 270, pp. 302311, 2011.

[176] J. Bauch und R. Rosenkranz, Physikalische Werkstoffdiagnostik, Springer Vieweg, 2017.

[177] D. A. Hills und D. W. Ashelby, „A note on shakedown,“ Wear, Nr. 65, pp. 125-129, 1980.

[178] J. S. Courtney-Pratt und E. Eisner, „The effect of a tangential force on the contact of metallic bodies," Proceeding of the Royal Society, pp. 529-550, 1957.

[179] T. F. J. Quinn, „Oxidational wear,“ Wear, Bd. 18, pp. 413-419, 1971.

[180] T. F. J. Quinn, „Review of oxidational wear Part II: Recent developments and future trends in oxidational wear research, "Tribology International, Bd. 16, Nr. 6, pp. 305315, 1983.

[181] T. F. J. Quinn und W. O. Winer, „The thermal aspects of oxidational wear, “ Wear, Bd. 102, pp. 67-80, 1985.

[182] D. A. Woodford und R. H. Bricknell, „Grain Boundary Penetration of Oxygen in Nickel and the Effect of Boron Additions," Metallurgical Transactions A, Bd. 12, Nr. 8, pp. 1467-1475, 1981. 
[183] T. H. Erismann, Prüfmaschinen und Prüfanlagen: Hilfsmittel der zerstörenden Materialprüfung, Springer-Verlag Berlin, Heidelberg 1992.

[184] J. D. Tiegom, Entwicklung eines wissensbasierten Systems über mechanische Werkstoff- und Bauteilprüftechnik, Bd. 36, W. Beitzs, Hrsg., Berlin: Technische Universität Berlin, Schriftenreihe Konstruktionstechnik, 1996.

[185] T. Kuttner, „Seminarskript Prüfstandstechnik in der Betriebsfestigkeit,“ 2013.

[186] W. Jorden, Form- und Lagetoleranzen, 3. Hrsg., Carl Hanser Verlag München Wien, 2005.

[187] R. P. Worthen und D. G. Rauen, „Valve parameters measured in firing engine, “ $S A E$, pp. 40-47, 1986.

[188] H. Steinbrenner und W. Pöll, „Bestimmung von Temperaturen an Ventilen,“ MTZ, pp. 47-50, 1961.

[189] H. C. Sin, N. Saka und N. P. Suh, „Abrasive wear mechanisms and the grit size effect,“ Wear, Nr. 55, pp. 163-190, 1979.

[190] A. Fischer, „Well-founded selection of materials for improved wear resistance, “ Wear, Bd. 194, Nr. 1-2, pp. 238-245, 1996.

[191] H. A. Sherif, „Effect of contact stiffness on the establishment of self-excited vibrations," Wear, Bd. 2, Nr. 141, pp. 227-234, 1991. 


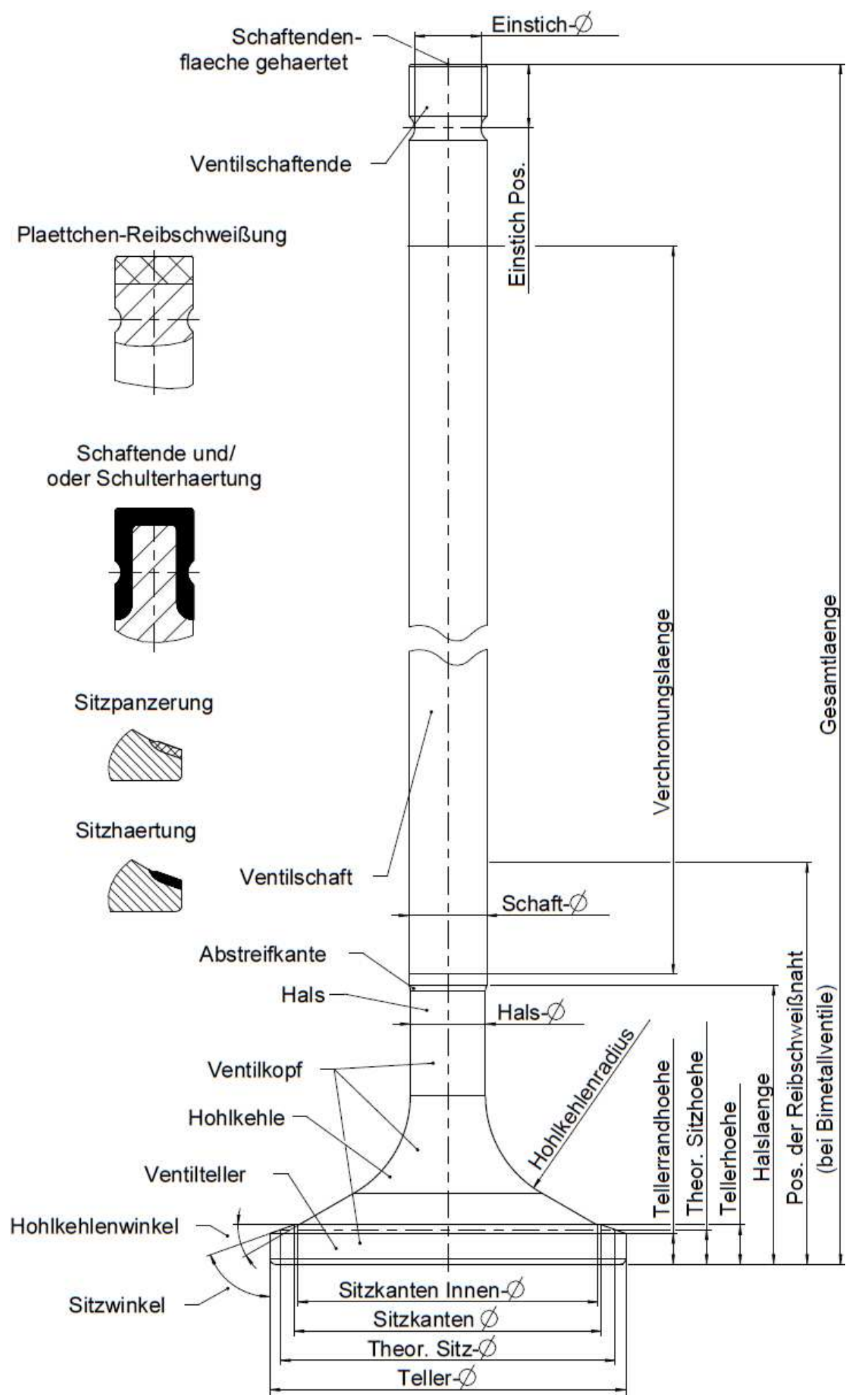

Abbildung 8.1: Bezeichnungen an einer Ventilspindel 


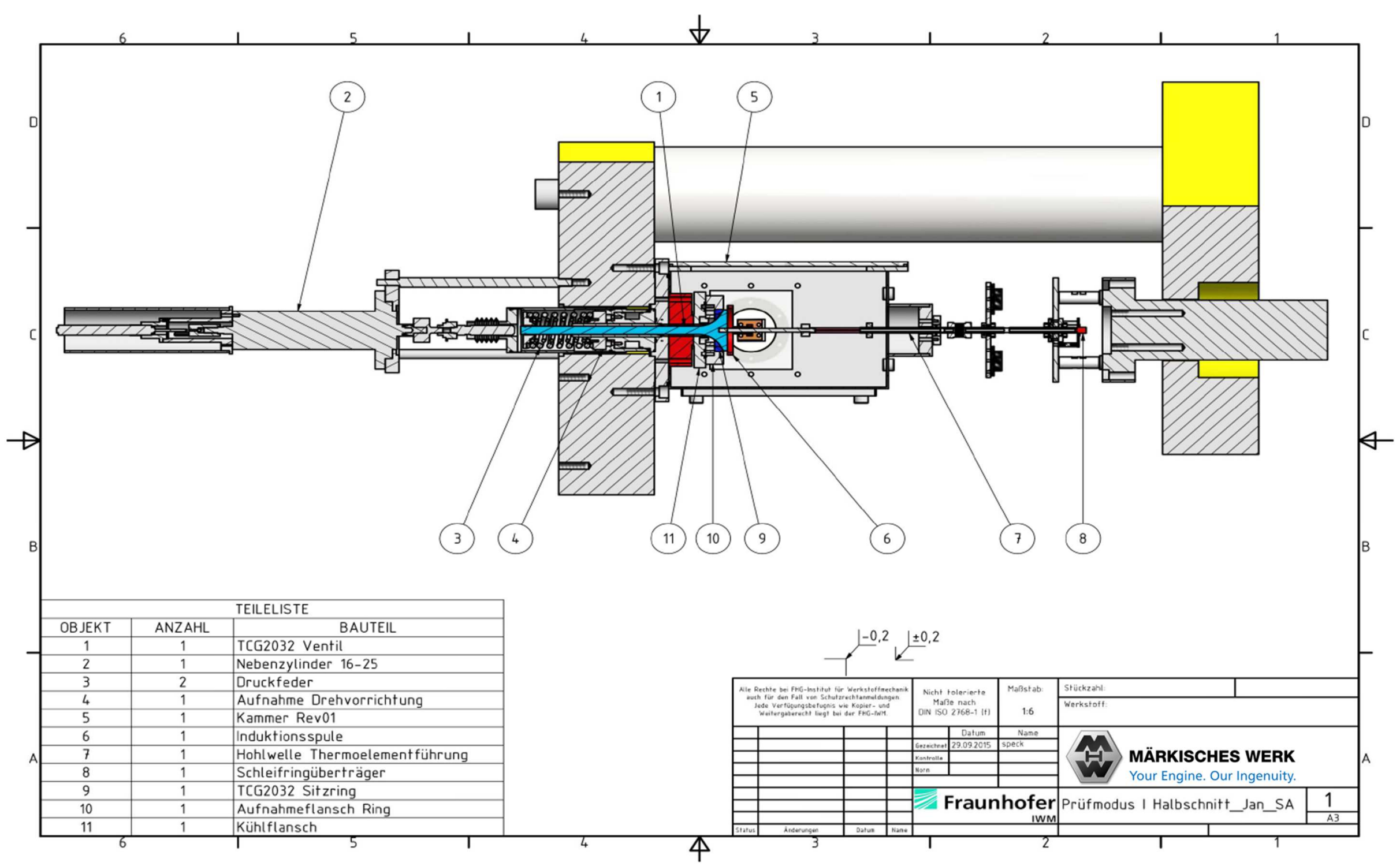

Abbildung 8.2: Zusammenbauzeichnung im Halbschnitt von Prüfmodus I 


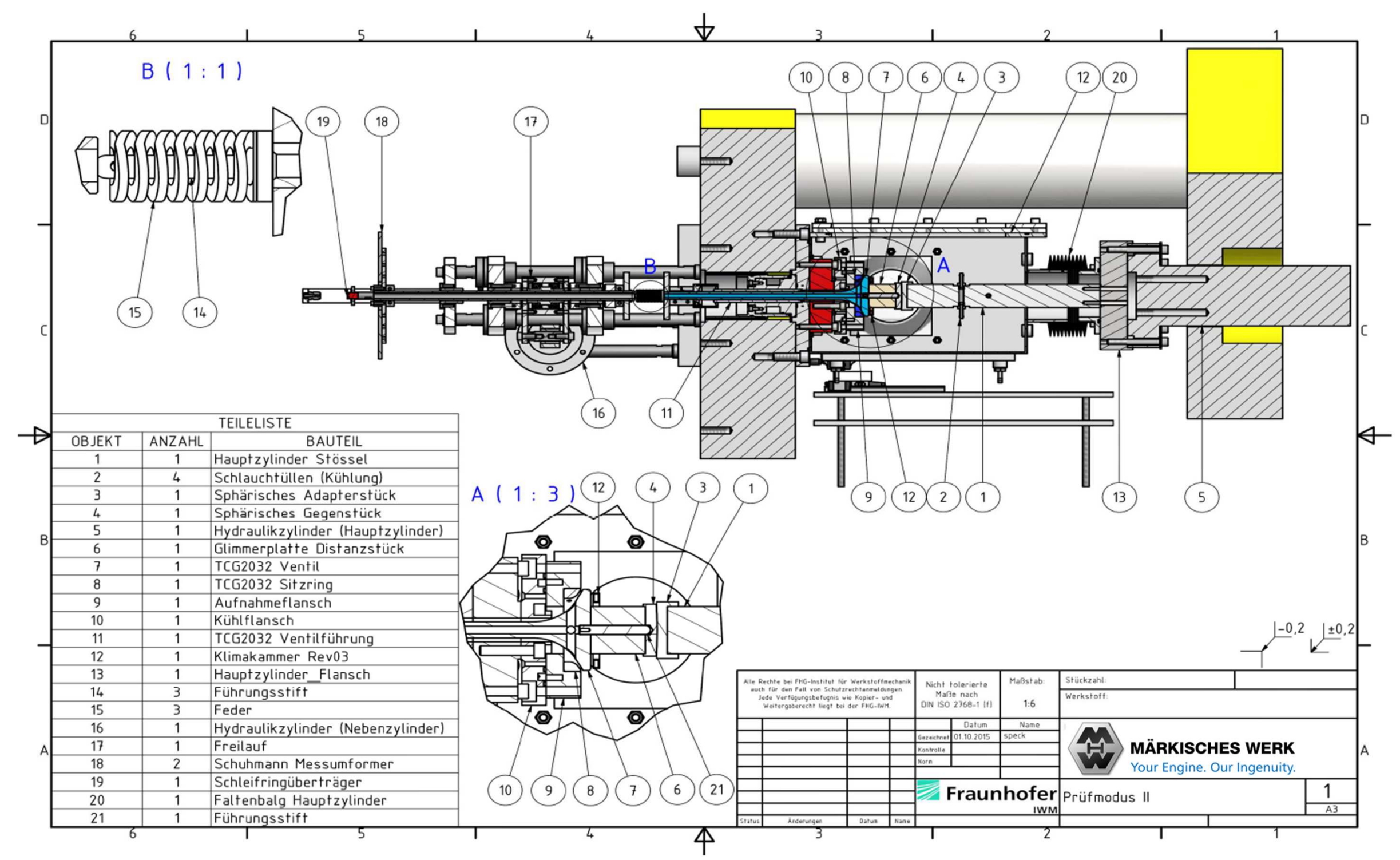

Abbildung 8.3: Zusammenbauzeichnung im Halbschnitt von Prüfmodus II 
